\title{
DIETARY PROTEIN INTAKE, BODY COMPOSITION AND SELF-REPORTED PHYSICAL FUNCTIONING IN U.S. ADULTS
}

\author{
A Thesis \\ presented to \\ the Faculty of California Polytechnic State University, \\ San Luis Obispo
}

\begin{abstract}
In Partial Fulfillment
of the Requirements for the Degree

Master of Science in Agriculture,
\end{abstract} with a specialization in Food Science and Nutrition

By

Kerri Beth Parker

September 2010 
(C) 2010

Kerri Beth Parker

ALL RIGHTS RESERVED 


\section{COMMITTEE MEMBERSHIP}

TITLE:

Dietary Protein Intake, Body Composition and

Self-reported Physical Functioning in U.S. Adults
AUTHOR:
Kerri Beth Parker
DATE SUBMITTED:
September 2010

COMMITTEE CHAIR: Louise Berner, Ph.D.

Professor

Food Science and Nutrition Department

COMMITTEE MEMBER: Jimmy A. Doi, Ph.D.

Associate Professor

Statistics Department

COMMITTEE MEMBER: Laura Marie Hall, Ph.D., RD

Assistant Professor

Food Science and Nutrition Department 


\section{ABSTRACT \\ Dietary Protein Intake, Body Composition and Self-reported \\ Physical Functioning in U.S. Adults \\ Kerri Beth Parker}

Sarcopenia is a growing health problem in this country as more Americans are living well into old age. It has been thought that a higher protein intake may be related to greater lean muscle mass as well as greater muscular strength and physical functioning. The purpose of this research was to examine protein intake and its relationship to lean body mass and physical functioning in a nationally representative sample of U.S. adults.

This research used cross-sectional data from the NHANES 2003-2004 to examine the relationship between protein intake, as an average from two $24 \mathrm{~h}$ recalls, and dual X-ray absorptiometry (DXA) lean body mass measures. Additionally, these lean body mass measures were examined as predictive of Physical Limitation Score (PLS), an index created from self-reported difficulty in performing basic activities of daily living.

Protein intake was examined as total grams of intake, a percentage of total daily energy and as a percent contributed by animal foods. The lean body mass measures used in this analysis included: total lean mass, appendicular lean mass, muscle mass index [lean mass / height $\left(\mathrm{m}^{2}\right)$ ], appendicular lean mass [appendicular lean mass / height $\left(\mathrm{m}^{2}\right)$ ] and percent lean body mass. Questions used to create the self-reported index of functioning, the Physical Limitation Score (PLS), included difficulty in independently: walking $1 / 4$ mile, walking up ten stairs without resting, lifting and carrying $10 \mathrm{lbs}$ and standing from an armless straight chair. 
It was found that protein intake significantly, positively predicted lean body mass in select age-gender groups, while more often in men than in women. Total grams of protein intake positively predicted total and appendicular muscle mass index in men ages 19-50 (p-value <0.05), appendicular muscle mass index in men ages 51-70 (p-value 0.038) and percent lean mass in men $71+$ years ( $\mathrm{p}$-value 0.026$)$. Protein as a percent of energy was a significant, positive predictor of appendicular lean mass in men 19-50 (pvalue 0.048), muscle mass index in women 19-50 (p-value 0.007), appendicular muscle mass index in women 19-50 (p-value 0.024) and percent lean mass in men $71+$ years ( $\mathrm{p}$ value 0.019). Protein as a percent of energy was a significant negative predictor of percent lean mass in older women 71+ years (p-value 0.046). Protein as a percent contributed by animal foods was not a significant predictor of lean mass in any agegender group.

It was also found that Physical Limitation Score (PLS) was surprisingly positively predicted by total and appendicular lean and total and appendicular muscle mass index in nearly all age-gender groups ( $\mathrm{p}$-value $<0.05$ ) or at least moderately, positively predicted by these ( $\mathrm{p}$-value $<0.10$ ), meaning that having a greater amount of lean mass predicted greater physical limitation. The only exception was percent lean mass, which was a significant negative predictor of PLS in men and women 60-70 years ( $\mathrm{p}$-value $<0.05$ ). In this case, a higher percent lean mass was associated with less physical limitation. Additional follow-up analyses revealed that total body fat mass $(\mathrm{kg})$ and percent body fat were significant positive predictors of PLS in most age-gender groups ( $\mathrm{p}$-value $<0.05$ ), indicating that higher amounts of body fat predicted greater self-reported limitation. 
Protein expressed as total grams per day or as a percent of total daily energy were generally significant or marginally significant predictors of lean mass in men of all ages but not often in women while protein as a percent contributed by animal foods was not found to be a significant predictor in any age-gender group. Total lean mass, total fat mass and total percent body fat were generally significant positive predictors of physical limitation, calling into question the functional implications of body composition in an older population. Results suggest that in older adults, excess body fat may be a stronger predictor of physical limitation than low lean muscle mass.

Keywords: Protein, Lean Mass, Physical Limitation, Sarcopenia, NHANES 


\section{ACKNOWLEDGEMENTS}

First and foremost, I would like to thank my committee chair, Dr. Louise Berner, for her leadership, direction and unyielding support throughout the past two years of my thesis research and compilation. I would also like to thank my committee members, Dr. Jimmy Doi for his statistical and programming assistance and Dr. Laura Hall for her continued advice and support throughout my research process and education as a Nutrition student.

I would like to thank Maxwell Wise and Brian Verbaken, both Cal Poly students in the Department of Statistics, for their invaluable programming assistance in the design and improvement of my statistical models. I also greatly appreciate the programming instruction of Rebecca Ottesen of the Cal Poly Department of Statistics, as the skills acquired during her SAS programming class provided me with the foundation I needed for the data management of my project.

I would also like to thank my family and friends for their continued encouragement and support of my research work and pursuit of my Master's degree.

Lastly, I would like to recognize and extend thanks to the California State University Agricultural Research Initiative, the Beef Checkoff, through the National Cattlemen's Beef Association, and the Dairy Council of California for their generous financial support of this research. 


\section{TABLE OF CONTENTS}

Page



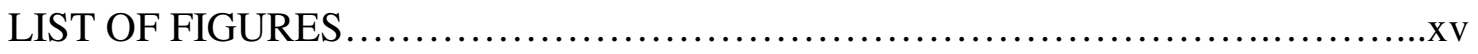

\section{CHAPTER}

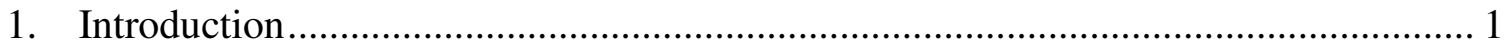

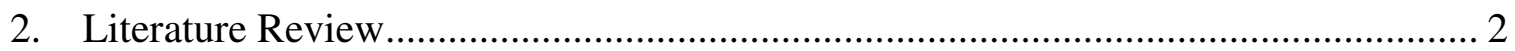

2.1. Current Protein Intake Recommendations in the United States.......................... 2

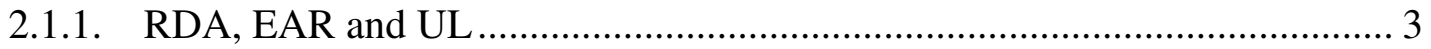

2.1.2. Acceptable Macronutrient Distribution Range (AMDR) .......................... 5

2.2. Controversy Regarding Current Protein Recommendations for Older Adults ..... 5

2.2.1. Measurement Tools for Protein Adequacy: Nitrogen Balance ..................... 5

2.2.1.1. Nitrogen Balance in Establishing Protein DRIs................................. 6

2.2.1.2. Shortcomings of Nitrogen Balance Methods ..................................... 9

2.2.1.3. Future Protein Assessment Tools: Direct Amino Acid Oxidation, 24hour Amino Acid Balance Test and Functional Measures of Health ... 10

2.2.2. Physiological Changes with Age and their Relationship to Protein Requirements in Older Adults .......................................................... 12

2.2.3. Risks Associated with Protein Toxicity ................................................. 15

2.3. Physical Measures of Health and Functioning in Older Adults........................ 19

2.3.1. Body Composition Changes with Age-Physiological and Financial

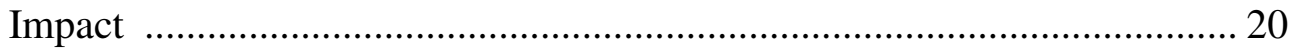

2.3.1.1. Sarcopenia Defined and Mechanisms Behind the Condition .............. 20

2.3.1.2. Functional and Health Implications of Reduced Lean Body Mass....... 25 
2.3.1.3. Financial Impact of Sarcopenia

2.3.1.4. Methods for Estimating Body Composition ..................................... 28

2.3.1.4.1. Anthropometry ................................................................. 28

2.3.1.4.2. Bioelectrical Impedance Analysis............................................ 35

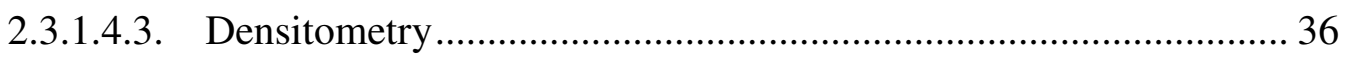

2.3.1.4.4. Dual X-ray Absorptiometry (DXA) ........................................ 38

2.3.2. Muscular Strength, Endurance and Quality and their Implications in

Older Adults ............................................................................... 41

2.3.2.1. Muscular Strength and Endurance ............................................... 41





2.4. Protein Intake and its Relationship to Body Composition, Muscular Strength, Endurance and Quality, and Other Physical Measures in Older Adults ............ 51

2.4.1. Current Protein Consumption Trends in the United States........................ 51

2.4.2. Relationship between Dietary Protein and Lean Body Mass in Older Adults ............................................................................................ 57

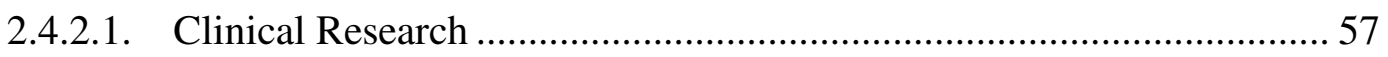

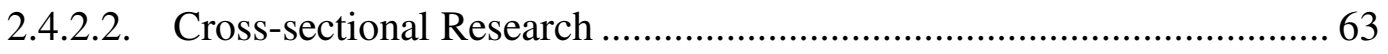

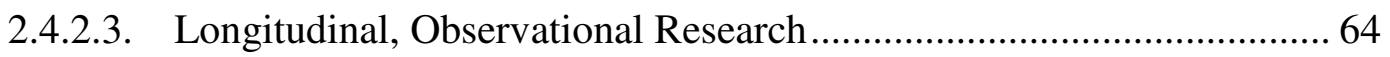

2.4.3. Relationship Between Dietary Protein and Muscular Strength, Endurance and Other Functional Measures of Health in Older Adults ........................ 66

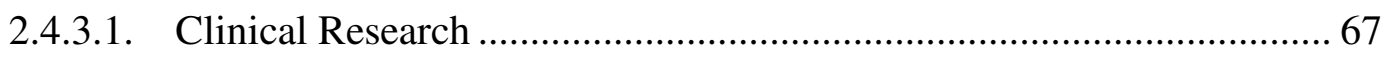

2.4.3.2. Cross-sectional Research ......................................................... 70

2.5. Justification and Practical Significance of Research ................................... 72

2.6. Main Research Questions and Hypotheses ............................................. 73 


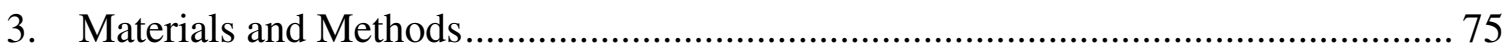

3.1. NHANES Collection and Management of Data ……….................................... 76

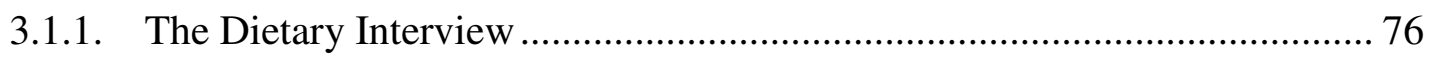

3.1.2. The Dual Energy X-ray Absorptiometry (DXA) Scan and Multiple

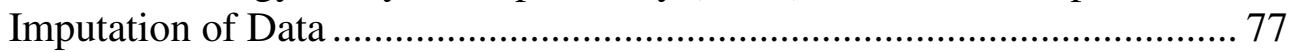

3.1.2.1. Justification for Multiple Imputation of DXA Data ............................. 78

3.1.2.2. Procedure for Multiply Imputing NHANES DXA Data........................ 80

3.1.3. The NHANES Physical Functioning Questionnaire.................................... 83

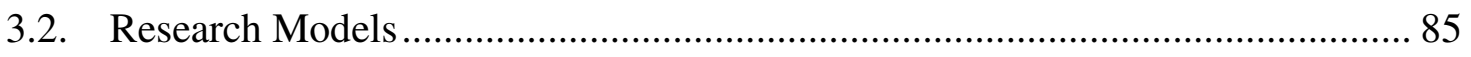

3.2.1. Lean Body Mass as Predicted by Protein Intake ........................................ 85

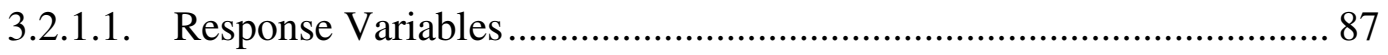



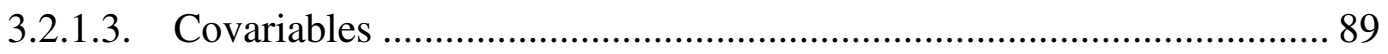

3.2.1.4. Summary of Analyses Run: Protein Intake as Predictive of Lean Body Mass ......................................................................................... 92

3.2.2. Self-reported Physical Functioning as Predicted by Lean Body Mass in

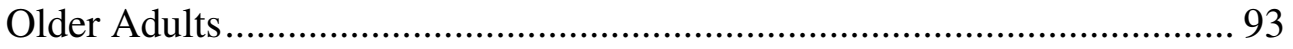

3.2.2.1. Response Variable ........................................................................ 95

3.2.2.2. Main Predictor Variables ..................................................................... 96

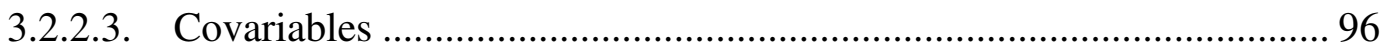

3.2.2.4. Summary of Analyses Run: Lean Body Mass as Predictive of Physical Functioning......................................................................... 98

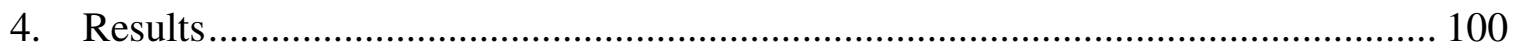

4.1. Descriptive Data..................................................................................... 100

4.2. Multiple Regression Analyses: Protein Intake as Predictive of Total Lean

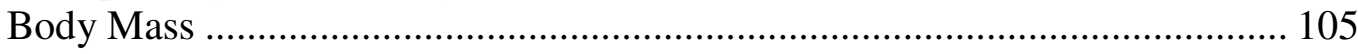


4.3. Multiple Regression Analyses: Protein Intake as Predictive of Appendicular Lean Body Mass

4.4. Multiple Regression Analyses: Protein Intake as Predictive of Total Muscle Mass Index $\left(\mathrm{g} / \mathrm{m}^{2}\right)$

4.5. Multiple Regression Analyses: Protein Intake as Predictive of Appendicular Muscle Mass Index $\left(\mathrm{g} / \mathrm{m}^{2}\right)$

4.6. Multiple Regression Analyses: Protein Intake as Predictive of Percent Lean Mass (\%)

4.7. Multiple Regression Analyses: Lean Body Mass as Predictive of Physical Limitation Score (PLS) ................................................................................. 144

5. Discussion, Conclusions and Directions for Future Research ................................. 155

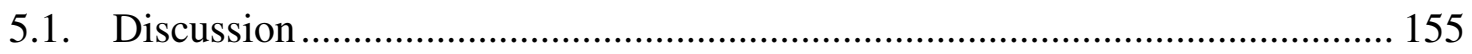

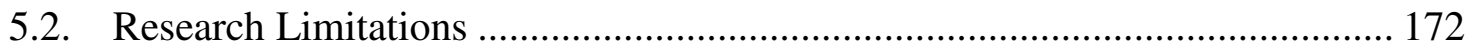

5.3. Conclusions and Directions for Future Research............................................... 173

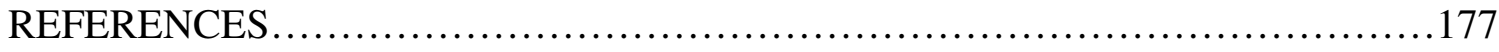
APPENDICES

A. DXA Variables Included in the Imputation Model.........................189

B. Non-DXA Variables Included in the Imputation Model......................190

C. NHANES 2003-2004 Physical Functioning Questionnaire- PFQ_C............193

D. Tables: Physical Limitation Score as Predicted by Body Fat Mass.............200 


\section{LIST OF TABLES}

Table

Page

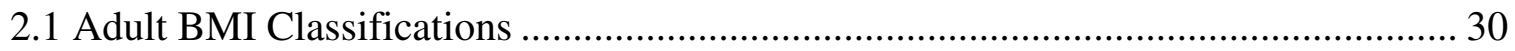

2.2 Grams of Protein Consumed per Day (g/day) and as a Percent of Daily

Energy Intake (\% en): Usual Intakes from Food Among Adults in the U.S.

2.3 Protein Intake ( $\mathrm{g} / \mathrm{kg}$ healthy body weight per day): Usual Intakes from Food Among Adults in the U.S. Data from the NHANES 2005-2006, 55

3.1 Percentages of NHANES participants from 1999 to 2004 with data missing, by age group.

3.2 Percentages of NHANES participants from 1999-2004 with data missing, by body mass index (BMI) category

4.1 NHANES 2003-2004 Descriptive Statistics: Protein intake in women.................... 101

4.2 NHANES 2003-2004 Descriptive Statistics: Protein intake in men........................ 102

4.3 NHANES 2003-2004 Descriptive Statistics: Lean body mass in women ............... 103

4.4 NHANES 2003-2004 Descriptive Statistics: Lean body mass in men ..................... 104

4.5 Total lean mass $(\mathrm{g})$ as predicted by total daily protein intake $(\mathrm{g})$ in women............ 107

4.6 Total lean mass $(\mathrm{g})$ as predicted by total daily protein intake $(\mathrm{g})$ in men................ 108

4.7 Total lean mass $(\mathrm{g})$ as predicted by protein as a percent of total daily energy $(\%)$

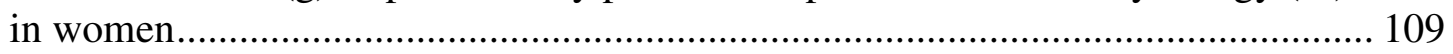

4.8 Total lean mass ( $\mathrm{g}$ ) as predicted by protein as a percent of total daily energy (\%) in men.

4.9 Total lean mass ( $\mathrm{g}$ ) as predicted by protein as a percent contributed by animal foods $(\%)$ in women.

4.10 Total lean mass ( $\mathrm{g}$ ) as predicted by protein as a percent contributed by animal foods $(\%)$ in men

4.11 Appendicular lean mass ( $\mathrm{g}$ ) as predicted by total daily protein intake $(\mathrm{g})$ in women 
4.12 Appendicular lean mass (g) as predicted by total daily protein intake (g) in men.

4.13 Appendicular lean mass ( $\mathrm{g}$ ) as predicted by protein as a percent of total daily energy $(\%)$ in women

4.14 Appendicular lean mass (g) as predicted by protein as a percent of total daily energy $(\%)$ in men .

4.15 Appendicular lean mass (g) as predicted by protein as a percent contributed by animal foods $(\%)$ in women

4.16 Appendicular lean mass (g) as predicted by protein as a percent contributed by animal foods $(\%)$ in men

4.17 Muscle Mass Index $\left(\mathrm{g} / \mathrm{m}^{2}\right)$ as predicted by total daily protein intake $(\mathrm{g})$ in women

4.18 Muscle Mass Index $\left(\mathrm{g} / \mathrm{m}^{2}\right)$ as predicted by total daily protein intake $(\mathrm{g})$ in men ... 124

4.19 Muscle Mass Index $\left(\mathrm{g} / \mathrm{m}^{2}\right)$ as predicted by protein as a percent of total daily energy $(\%)$ in women

4.20 Muscle Mass Index $(\mathrm{g} / \mathrm{m} 2)$ as predicted by protein as a percent of total daily energy $(\%)$ in men .

4.21 Muscle Mass Index $\left(\mathrm{g} / \mathrm{m}^{2}\right)$ as predicted by protein as a percent contributed by animal foods $(\%)$ in women

4.22 Muscle Mass Index $\left(\mathrm{g} / \mathrm{m}^{2}\right)$ as predicted by protein as a percent contributed by animal foods $(\%)$ in men

4.23 Appendicular Muscle Mass Index $\left(\mathrm{g} / \mathrm{m}^{2}\right)$ as predicted by total daily protein intake $(\mathrm{g})$ in women.

4.24 Appendicular Muscle Mass Index $\left(\mathrm{g} / \mathrm{m}^{2}\right)$ as predicted by total protein intake $(\mathrm{g})$ in men

4.25 Appendicular Muscle Mass Index $\left(\mathrm{g} / \mathrm{m}^{2}\right)$ as predicted by protein as a percent of total daily energy (\%) in women.

4.26 Appendicular Muscle Mass Index $\left(\mathrm{g} / \mathrm{m}^{2}\right)$ as predicted by protein as a percent of total daily energy $(\%)$ in men.....

4.27 Appendicular Muscle Mass Index $\left(\mathrm{g} / \mathrm{m}^{2}\right)$ as predicted by protein as a percent contributed by animal foods (\%) in women 
4.28 Appendicular Muscle Mass Index $\left(\mathrm{g} / \mathrm{m}^{2}\right)$ as predicted by protein as a percent contributed by animal foods $(\%)$ in men

4.29 Percent lean mass $(\%)$ as predicted by total daily protein intake $(\mathrm{g})$ in women.... 138

4.30 Percent lean mass $(\%)$ as predicted by total daily protein intake $(\mathrm{g})$ in men

4.31 Percent lean mass (\%) as predicted by protein as a percent of total daily energy $(\%)$ in women

4.32 Percent lean mass (\%) as predicted by protein as a percent of total daily energy $(\%)$ in men

4.33 Percent lean mass (\%) as predicted by protein as a percent contributed by animal foods $(\%)$ in women

4.34 Percent lean mass (\%) as predicted by protein as a percent contributed by animal foods $(\%)$ in men

4.35 Physical Limitation Score (PLS) as predicted by total lean mass (kg)

4.36 Physical Limitation Score (PLS) as predicted by appendicular lean mass (kg) ..... 147

4.37 Physical Limitation Score (PLS) as predicted by Muscle Mass Index $\left(\mathrm{kg} / \mathrm{m}^{2}\right) \ldots . . .149$

4.38 Physical Limitation Score (PLS) as predicted by Appendicular Muscle Mass Index $\left(\mathrm{kg} / \mathrm{m}^{2}\right)$

4.39 Physical Limitation Score (PLS) as predicted by percent lean mass (\%)

5.1 Summary of findings: Statistical significance of dietary protein (g/day) as a predictor of lean body mass measures in women and men 


\section{LIST OF FIGURES}

Figure

Page

2.1 EWGSOP-Suggested Algorithm for Sarcopenia Case Finding in Older

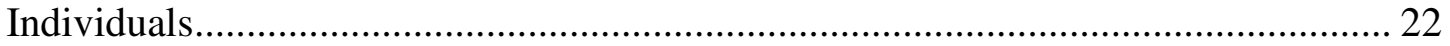

2.2 Interrelated Mechanisms of Sarcopenia............................................................. 24

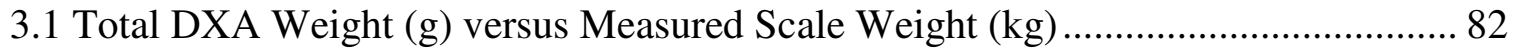




\section{Introduction}

The preservation of lean muscle mass and the maintenance of independent physical functioning are among the most important issues affecting quality of life as an older adult. Sarcopenia, a progressive loss of skeletal mass and strength, is estimated to affect more than 50 million older adults worldwide and poses an associated healthcare cost upwards of \$18.5 billion in the U.S. alone (Cruz-Jentoft et al. 2010; Janssen et al. 2004).

There is evidence that protein intake may play a role in the preservation of lean body mass and muscular strength with age but there is limited data available that examines this relationship in a nationally representative sample of older adults. Furthermore, there is limited data available that looks at the direct relationship between lean body mass and physical functioning in such a sample.

The purpose of this cross-sectional research was to examine the distributions of and relationships between protein, lean body mass and self-reported physical functioning through the analysis of data from a large, nationally representative sample of adults from the NHANES 2003-2004. While the cross-sectional nature of this research does not allow for conclusions of cause and effect, results will be useful in assessing current distributions of protein intake and lean mass in adults 19 years and older. In addition, it will reveal whether greater intake of protein is significantly predictive of lean body mass and whether lean body mass significantly predicts self-reported physical functioning. Results from this research will lay the groundwork and strengthen the case for further longitudinal, observational and intervention studies in older adults. 


\section{Literature Review}

Together as a collection of individual amino acids, proteins serve as vital, multi-

faceted tools within the human body, providing all cells and enzymes with functional and structural support while serving as major components of hair, fingernails, serum albumin, keratin and collagen in bones, ligaments and tendons. Separate from whole proteins, individual amino acids function as precursors to hormones, coenzymes, nucleic acids and other molecules essential to life. The cellular roles of proteins function together at the whole-body level to build and maintain lean tissue while perpetuating human growth and reproduction and promoting strength and proper physical functioning.

As humans age, lean tissue mass often begins to decline, resulting in a loss of physical functioning and an increased susceptibility to falls, fractures and daily dependency. This condition of muscle wasting, termed sarcopenia, affects thousands of older Americans but is only partially understood and ambiguously defined. Researchers question whether sarcopenia is, in fact, a disease state or merely a natural, inevitable part of aging; in addition, questions remain regarding the role of dietary protein in preserving the integrity of lean tissue mass, both anthropometrically and functionally. These issues remain areas of disparity among researchers and, as a result, serve as topics of current research and investigation (Chernoff 2004; Roberts et al. 2005; Lord et al. 2007).

\subsection{Current Protein Intake Recommendations in the United States}

Protein's roles as a muscle-builder and key component of nearly every tissue in the human body more than justify the need for the establishment of daily consumption guidelines for this macronutrient. From 1997 to 2005, a Food and Nutrition Board 
committee within the Institute of Medicine of the National Academy of Sciences worked to expand the original Recommended Dietary Allowances (RDAs) that had been established and revised multiple times between 1941 and 1989 (IOM, 2002). This new group of nutrient-based dietary reference values, known collectively as the Dietary Reference Intakes (DRIs), set new recommended intakes for all macronutrients, total energy, water, physical activity, vitamins and minerals. These reference intakes were meant to serve as direct tools for health professionals of the United States and Canada in the assessment and planning of diets of individuals within the general population. The DRIs for protein were released in 2002 and include both estimated average requirements (EARs) and an associated Recommended Dietary Allowance (RDA) for this macronutrient (IOM 2002).

\subsubsection{RDA, EAR and UL}

The adult EAR for protein is set at 0.66 grams per kilogram of body weight per day. This national recommendation, derived from a meta-analysis of 19 primary nitrogen balance studies, is defined as the average level of protein intake estimated to achieve nitrogen balance in half of the healthy individuals in a particular life stage or gender group (IOM 2002). The EAR represents an estimated median requirement for intake and reflects a nutrient amount that should meet the needs of half of a particular group but fall short of the needs of the other half. It is important to note that the EAR is not meant for use as a goal daily intake for individuals, rather as a tool for assessing the adequacy of a nutrient within a population, reported as the percent of a population with an intake above or below the EAR (Gibson 2005). 
The Recommended Dietary Allowance (RDA) for protein is more widely recognized and utilized as a goal intake for individuals. It represents the average daily dietary nutrient intake level sufficient to meet the nutrient requirements of nearly all (97-98 percent) of healthy individuals in a particular life stage and gender group (IOM 2002). The current adult RDA for protein is set at 0.80 grams of dietary protein per kilogram of body weight per day or 46 grams of protein per day for women and 56 grams per day for men (based on reference body weights of 57 and 70 kilograms, respectively). It is important to note that these reference weights are relatively low and atypical in America today. Consequently, following the RDA guideline based on individual body weight may provide the most appropriate way for estimating individual requirements (Gibson 2005). The IOM used the same 19 primary nitrogen balance studies in determining this recommendation; for standard normal nutrient distributions, the RDA is essentially the EAR plus two standard deviations. Thus, it reflects a value estimated to meet the needs of a much greater proportion of the population but exceeds the recommendations of nearly all members of a life stage or gender group (Gibson 2005).

The Tolerable Upper Level (UL) is a DRI value defined as the highest level of nutrient intake that is likely to pose no risk of adverse health effects for almost all individuals in a specified life stage group. As intake increases above the UL, there is potential for an increased risk of adverse health effects. While no UL has been set for protein, caution must still be taken in consuming amounts well in excess of the recommended intake, as this missing value merely indicates that currently there is insufficient evidence to warrant the establishment of a potentially harmful level of 
consumption. As investigation continues, there is always potential that researchers will find reason for the establishment of a protein UL (IOM 2002).

\subsubsection{Acceptable Macronutrient Distribution Range (AMDR)}

Another dietary guidance tool, useful when applied in conjunction with the RDA, is the Acceptable Macronutrient Distribution Range (AMDR). The AMDR for protein, set as an intake between 10 and $35 \%$ of total daily energy, is not in itself a goal intake. Rather, this range of intake may be associated with a decreased risk of chronic disease as long as RDA parameters are also being met (IOM 2002).

\subsection{Controversy Regarding Current Protein Recommendations for Older Adults}

The Dietary Reference Intake guidelines for all nutrients are constantly under scrutiny and revision in an effort to keep up with ongoing food and nutrition research. As mentioned previously, the RDA for protein is broadly set for all adults, without regard to age or gender. Due to methods used in establishing the RDA and physiological changes that occur with age, there is controversy among researchers as to whether this recommendation requires further stratification to match the specific needs of older versus young adults.

\subsubsection{Measurement Tools for Protein Adequacy: Nitrogen Balance}

It is assumed that, since nitrogen is present in the body almost exclusively as a part of proteins, its turnover serves as an accurate predictor of whole-body protein anabolism and catabolism. Hence, DRIs for dietary protein are derived primarily from nitrogen balance studies during which total nitrogen losses from urine, feces, skin and sweat, 
mainly in the form of urea, are subtracted from total nitrogen dietary intake. In nitrogen balance techniques it is assumed that, where dietary protein needs are met or exceeded, adults will come into zero or positive nitrogen balance respectively, while inadequate intakes will result in a negative balance (Rand et al. 2003).

The process of nitrogen balance testing generally involves the measurement of urinary nitrogen, primarily in the form of urea, a byproduct of partial protein oxidation. Nitrogen is also lost from the body in feces, hair, skin sloughing and excessive sweating. While researchers occasionally measure these other pathways directly, the high cost and elaborate nature of such testing more often results in measurement of only urinary nitrogen while standard estimates for these other, less significant losses are employed (Rand et al. 2003). Milward and Roberts, in their review of the literature, concluded that $\leq 5 \mathrm{mg} \mathrm{N}$ per kilogram of body weight per day serves as an accurate estimate of dermal and miscellaneous nitrogen losses (Millward \& Roberts 1996). However, research has also shown that this value varies based on climate as well as the amount of nitrogen consumed and that a single, broad estimate of losses may not be entirely appropriate (Rand et al. 2003). While N-balance techniques often include only the direct measurement of urinary nitrogen and are associated with several other shortcomings, to be discussed later, this method remains the chosen approach for setting national protein recommendations, mainly due to the lack of a validated or accepted alternative (Rand $e t$ al. 2003).

\subsubsection{Nitrogen Balance in Establishing Protein DRIs}

The data analyzed in the meta-analysis of nitrogen balance studies used to set national dietary protein recommendations show no statistical differences that warrant unique 
protein requirements based on climate, gender, common protein source or age. Hence, requirements for older adults and the elderly 50 years and over remain identical to recommendations for younger adults (Rand et al. 2003). It is important to note, however, possible reasons for these findings of no statistical difference. First of all, there was only one study that reported individual data on requirements for older subjects, two sub-studies of men and two groups of women. In addition to this, the data obtained from all studies do not provide sufficient statistical power to detect more than very major differences in requirements (Rand et al. 2003). In fact, when the efficiency of nitrogen utilization for retention was studied researchers $d i d$ find a significantly lower efficiency in older individuals (p-value 0.003), suggesting a possible age-related decrease in nitrogen utilization and retention, likely due to infection, trauma or disease; however, this finding was deemed inconclusive due to the low number of elderly subjects used in the analysis (Rand et al. 2003).

Further limiting the conclusions of the nitrogen balance studies included in this review is the fact that most did not control for total energy intake or physical activity. Both are hypothesized to have an effect on nitrogen and protein metabolism; in fact, researchers were able to conclude that approximately one-third of the variation in nitrogen balance among individuals could be explained by differences in energy intake (Rand et al. 2003). Between the 19 total studies used, it is likely that methodological differences in determining nitrogen requirements as well as innate variability within and between test subjects may have affected the outcome of the analysis (Rand et al 2003).

There have been a number of more recent nitrogen balance studies conducted on older adults in an attempt to assess the adequacy of the RDA for this age group. 
Campbell and colleagues, in a recent clinical assessment of protein adequacy in younger and older adults via short-term nitrogen balance testing at three different levels of protein intake $(0.50,0.75,1.0 \mathrm{~g} / \mathrm{kg} / \mathrm{day})$, concluded that the mean protein requirement was not different between younger and older subjects as the amount needed to achieve nitrogen balance did not differ significantly (Campbell et al. 2008). In his study, Campbell collected urine and stool samples along with 12 days of duplicate portions of all energycontaining foods and beverages to more accurately measure nitrogen intake and excretion. This study is believed to provide the most comprehensive nitrogen balancebased assessment of protein needs for older men and women ever published and concludes that the RDA of $0.8 \mathrm{~g} / \mathrm{kg} / \mathrm{day}$ is adequate to meet the minimum dietary needs for short-term nitrogen balance in virtually all older persons (Campbell et al. 2008).

Contrary to Campbell, another short-term (3-week) nitrogen balance study assessing the adequacy of the protein RDA by testing elderly women at the same three levels of protein intake $(0.50,0.75,1.0 \mathrm{~g} / \mathrm{kg} / \mathrm{day})$, concluded that, although a metabolic steady state was not achieved, the total protein needs for this group were at or above the current RDA of $0.8 \mathrm{~g} / \mathrm{kg} / \mathrm{day}$ (Morse et al. 2001). Similarly, in a cross-sectional evaluation of nitrogen balance in 36 elderly hospital patients, researchers found that energy and protein intakes correlated positively with nitrogen balance and that mean protein intake to reach a neutral nitrogen balance was approximately $1.06 \mathrm{~g} / \mathrm{kg} / \mathrm{day}$, again significantly higher than the current adult RDA (Gaillard et al. 2008).

While the DRI committee did not make use of many N-balance studies using elderly adults when establishing current protein intake recommendations, continuing research within this age group may lead to updated DRIs stratified by age in coming years. 


\subsubsection{Shortcomings of Nitrogen Balance Methods}

Aside from the high probability of variability between tests and test subjects, the nitrogen balance method itself has a number of substantial, practical limitations. In adults, it has been established that the rate of urea turnover, the main nitrogen-containing end-product of protein metabolism, is very slow and requires several days of adaptation at each new protein level tested to attain a new steady state of nitrogen excretion (Rand et al. 2003). In one relatively large feeding experiment designed to assess the adequacy of the EAR for older adults via nitrogen balance methods, researchers were able to conclude that there were no significant differences in protein needs between the younger and older groups tested. However, in the group of older women being tested, urinary nitrogen excretion increased significantly from week 2 to week 3 indicating the need for longer periods of adaptation to a protein intake to obtain accurate results. Most studies, including those used to set current RDAs, were conducted on a very short-term basis (Campbell et al. 2002).

To more accurately estimate dermal and miscellaneous losses and to predict requirements for individuals, it is imperative to test the same person across different levels of nitrogen intake rather than testing across groups of individuals, as is commonly done. Dermal and miscellaneous losses from such avenues as hair, sweat and teethbrushing vary widely between individuals due to environmental conditions and each person's unique physiology, thus increasing the risk for inaccurate conclusions drawn from cross-sectional N-balance studies (Rand et al. 2003).

Nitrogen balance testing does have its shortcomings but is still used as an assessment of protein adequacy, even more so in recent studies. However, Rand and colleagues 
highlight the need for additional protein assessment tools as they conclude that, "Maintenance of a measured nitrogen balance does not necessarily imply an equivalent maintenance of nitrogen balance or of protein and amino acid function in all organs and tissues. Other measures of both organ and whole-body protein adequacy are needed and the fields of molecular biotechnology, proteomics and metabolomics need to be exploited in the search for new paradigms for nutrient requirement studies" (Rand et al. 2003).

\subsubsection{Future Protein Assessment Tools: Direct Amino Acid Oxidation, 24-hour Amino Acid Balance Test and Functional Measures of Health}

It is possible that the studies used to determine national protein recommendations and purport the homogeneity in requirements across the life span have fallen prey to the pitfalls and practical limitations of the nitrogen balance method. There are wellestablished and proven physiological, psychological and social changes that occur within the human body as it ages that may warrant the need for additional assessments of protein adequacy. At the 2008 American and European Protein Summit, experts reached a major consensus regarding nitrogen balance and its lack of direct correlation with functional outcomes. Therefore, it may not be the most valid and appropriate tool for the determination of optimal levels of protein intake. Researchers at the summit didn't propose to throw out the technique entirely, rather to incorporate additional protein adequacy indicators related to muscle mass, strength and metabolic function (Wolfe 2008).

In addition to the functional measures proposed at the 2008 protein summit, several newer, clinical methods for assessing protein adequacy are slowly pushing their way into the limelight, with a particular focus on indicators to assess the adequacy of individual, 
indispensable amino acids. In direct amino acid oxidation methods, researchers are able to measure the carbon oxidation of single indispensable amino acids. For this procedure, the test amino acid is labeled with carbon and the production of breath $\mathrm{CO}_{2}$ serves as a measure of oxidative loss of that amino acid (IOM 2002). Drawbacks to this method, however, include the reliance on a set "break point" of consumption above which oxidation increases progressively, a value that can be accurately set only by administering very low doses of the amino acid. Also, studies that have been done using this method have only measured oxidation during a short period thus they are not representative of the day as a whole. This method is also limiting in that it can only be used to assess the capacity of branched chain amino acids as opposed to total protein adequacy (IOM 2002).

This method improved and evolved into a 24-hour amino acid balance test that requires measurements taken at more regular intervals throughout the day in both fasting and fed states. This procedure theoretically allows for the direct measurement of the amounts of specific amino acids lost under different nutritional conditions. As of now, only leucine, lysine and phenylalanine have been studied using this method (IOM 2002).

The assessments of physical measures of health provide simpler and potentially more functionally applicable tests for nutritional adequacy. Strength, ambulatory ability and the extent to which a person can meet the demands of daily life serve as examples of such functional measures. The limitations of nitrogen balance techniques used to set national protein recommendations highlight a need for the implementation of additional tests of protein adequacy. As further research is yet to be done, leading nutrition experts remain 
in a state of disagreement as to where adequate protein recommendations should lie and whether these should be differentially stratified across age groups.

\subsubsection{Physiological Changes with Age and their Relationship to Protein Requirements in Older Adults}

In addition to questions regarding the validity of nitrogen balance techniques and due to proven physiological changes with age, researchers remain in a state of controversy with respect to national protein recommendations and their adequacy in meeting the needs of older adults. Changes in body composition, as seen in altered ratios of protein, water, fat and bone, is one of the most notable characteristics of human aging. As people grow older bone density, muscle mass and total body water naturally tend to decrease, while total body fat, both inter- and intra-muscularly, generally increase proportionally (Chernoff 2004). The term "sarcopenic obesity" explains this condition in which muscle wasting problems associated with aging are often masked by simultaneous increases in adipose tissue. The fat shift from subcutaneous storage to abdominal and intramuscular locations has several negative consequences, primarily decreased insulin sensitivity, thereby increasing the risk of developing Type 2 Diabetes (Volpi et al. 2000).

In an experimental study comparing the response of muscle protein anabolism to combined hyperaminoacidemia and glucose-induced hyperinsulinemia in the young and elderly, researchers found a significant decrease in blood flow to the leg upon ingestion of an amino acid-glucose supplement in the older subjects (72 $\pm 1 \mathrm{yr}$.). This phenomenon was not seen in younger adults and may reflect a reduced response of the elderly body to insulin (Volpi et al. 2000). According to researchers from this same study, these findings are consistent with others that report reduced blood flow upon infusion of insulin, as 
opposed to the vasodilatory response commonly seen in young, healthy adults. Reduced sensitivity to insulin, a key player in postprandial protein anabolism and reduced proteolysis, could potentially serve as a main contributor to losses of lean tissue with age. The central question then becomes whether an increased protein intake in older adults is necessary to induce a comparable insulin response to that of their younger counterparts and, in turn, to maintain relatively equal amounts of lean tissue preservation and anabolism (Volpi et al. 2000).

The aging body also changes noticeably at the cellular level, further supporting the need for an increased protein requirement in older adults. Within skeletal muscle, older adults experience both a loss and decreased size of muscle fibers as a result of muscular dehydration and decreased mitochondrial number, resulting in a decline in total muscle mass, size and strength (Thornell et al. 2003). There is also evidence of reduced speed and strength of the cross-bridging action of muscle filaments, particularly in the myosin heavy chain, leading to a slower whole muscle contractile speed and resulting in impaired mobility and physical reaction time (Thornell et al. 2003).

Aged muscle fibers are also susceptible to losses in satellite cells—-small mononuclear cells that fuse with the sarcolemma of a mature muscle fiber and donate a nucleus, aiding in cell growth and function as well as regeneration following injury or disease. Satellite cells are capable of proliferating under conditions of muscle injury, stress or strain to grow new muscle fibers. Thus, fewer numbers of these protective cells can partially account for whole- body impairment of wound healing and recovery from exercise and disease (Thornell et al. 2003). 
In one small study, researchers examined the effects of aging on changes in human muscle via biopsies of the vastus lateralis muscle of physically untrained men, eight young and eight old. Conclusions from this study include significant decreases in both the number of and total muscle area occupied by Type 2 muscle fibers in the elderly when compared with the young men. In addition, researchers observed a significant reduction in the number of satellite cells in elderly Type 2 muscle fibers, even after adjustment for decreased muscle cross-sectional area. Such differences were not observed in Type 1 fibers. This study points toward a strong correlation between satellite cell number and myofiber size, suggesting that reductions in these protective cells play an important role in the degeneration of muscle (Verdijk et al. 2009).

Aging is also generally associated with increased levels of oxidative stress, shown to cause damage to muscle cell membranes and proteins. This damage has been associated with a wide range of chronic diseases as the body is unable to initiate a defense against cellular attack. Glutathione, a major antioxidant associated with the proliferation of lymphocytes and part of the metabolic functioning of nearly all cells, may also be affected by inadequate intakes of certain amino acids; namely, cysteine, glutamate and glycine. Thus, greater amounts of dietary amino acids may work in the elderly to prevent excessive cellular damage (Dawson et al. 2008).

Aside from changes within the muscle fiber, older persons also experience a reduction in other protein compartments within the body. These include red and white blood cells, blood platelets, stem cells, antigens, antibodies, hormones, and enzymes (Chernoff 2004). Along with shifts in body composition, these changes have been shown to contribute to impaired wound healing, a weakened immune system, muscle weakness 
and an inability to maintain all-around tissue integrity, all resulting in impaired functioning at the whole-body level (Chernoff 2004).

The question remains, would an increased recommendation for dietary protein in older adults help alleviate some of these age-related detrimental changes? It has been hypothesized by researchers that an increased protein requirement is necessary to heal wounds, fight infection and replace tissue losses in aging however, as people age they also experience a decrease in resting metabolic rate and in overall energy needs. Does it really make sense to recommend dietary intakes equivalent to those of younger, more physically and metabolically-active adults? According to Paddon-Jones and fellow researchers and as stated before, older people may experience a lesser anabolic response after a mixed nutrient meal, increasing the protein requirements necessary to garner the same muscle-building effect of a younger person (Paddon-Jones et al. 2004a). In another study by Volpi and colleagues, researchers measured protein synthesis, breakdown and amino acid transport during intramuscular infusion of an amino acid mixture in both young and elderly subjects. They concluded that, even though muscle mass was decreased in the elderly as compared with younger adults, muscle anabolism could be stimulated when amino acid availability was increased (Walrand \& Boirie 2005). Thus researchers showed that, while older adults may be less sensitive to protein, increased amounts might provide an anabolic effect similar to that of their younger counterparts.

\subsubsection{Risks Associated with Protein Toxicity}

While many studies advocate the need for an increased protein requirement for the elderly, others are quick to point out potential pitfalls when consuming too much. As mentioned previously, no Tolerable Upper Level has been set for protein but this does not 
mean that excessive consumption of this nutrient poses no potential health threats. It simply makes the statement that, at this time, we do not have sufficient evidence to establish a maximum, safe value. When considering the DRIs as mentioned before, the RDA of $0.8 \mathrm{~g} / \mathrm{kg}$ of body weight remains the goal level of intake.

The greatest concerns regarding the over-consumption of protein involve increased calcium excretion and increased stress on the kidneys. There is reasonable certainty that calcium excretion increases with increased protein intake. It has been proposed that calcium excretion can increase by as much as $50 \%$ when protein intake doubles and all other nutrients are held constant (Heaney 1998). The question then becomes, does this increase in excretion negatively affect bone and play a contributing role in the development of osteoporosis and will the high concentration of calcium in the urine increase the production of kidney stones?

Heaney (1998) suggests that, rather than looking for correlations between protein intake and bone mineral density, researchers should focus on individual calcium to protein ratios in the diet, as increases in protein intake are often associated with higher absorption of food calcium. According to Heaney, the body, in an attempt to adapt to a drop in extracellular fluid $[\mathrm{Ca}]$ in response to a high protein meal, increases the secretion of parathyroid hormone, thereby positively affecting calcium absorption.

In one controlled feeding study during which a group of post-menopausal women were given high and low calcium diets in combination with both high and low protein diets (10\% and $20 \%$ protein, respectively), researchers found the women, when on the higher protein diet, slightly improved calcium absorption from a low-calcium diet, nearly compensating for a slight increase in urinary calcium excretion (Hunt et al. 2009). In this 
study, increased dietary protein from animal sources was not detrimental to calcium balance or short-term indicators of bone health. Thus, it may be true that the effect of a high protein diet on bone health is dependent upon the adequacy of calcium intake.

Based on the DRIs for each nutrient, researchers from this study propose a recommended calcium to protein ratio of 20:1 or 20 milligrams of calcium: 1 gram of protein (Hunt et al. 2009).

In addition to questions regarding calcium excretion and changes in bone mineralization come concerns regarding the effect of high concentrations of urinary calcium on overall kidney function and the production of kidney stones. An extremely high protein intake may overwork the kidneys, as they have to filter and excrete the excess nitrogen, mainly in the form of urea. The most common form of kidney stone is composed mainly of calcium and oxalate, both suspected to increase in concentration in the urine with high protein consumption. In one large, prospective study investigating potential causes of kidney stones, researchers found that that a high animal protein intake in healthy humans increases urinary calcium and oxalate and the overall probability of forming kidney stones by 250 percent (Curhan et al. 1993).

There is also some concern regarding high protein intake and its role in the development or progression of renal failure, although much disagreement remains surrounding this theory. It has been proposed that excessive protein intake may affect glomerular filtration rate (GFR), the amount of filtrate formed per minute by the two kidneys combined, thereby causing problems with nutrient and water reabsorption. GFR is the standard clinical measure of renal function with reference healthy values of $105 \mathrm{ml} / \mathrm{min}$ and $125 \mathrm{ml} / \mathrm{min}$ in females and males respectively. If this value is too high, 
fluid flows through the renal tubules too quickly for nutrients to be absorbed, resulting in dehydration and electrolyte imbalances. However, if the GFR ends up too low, the slower moving fluid allows too much time for reabsorption, and wastes that should be eliminated in the urine remain in the body (Saladin 2004). Many researchers theorize that increases in protein intake generally increase GFR, possibly to dangerous levels.

In one study involving a group of ten older subjects and ten younger subjects, researchers found that, in the younger group fed a very high protein diet of 3.0 grams $/ \mathrm{kg}$ fat-free mass (more than twice the protein RDA) for ten days, GFR significantly increased, from a baseline of $87-122 \mathrm{ml} / \mathrm{min}$ to an end range of $113-172 \mathrm{ml} / \mathrm{min}$. However, in older adults consuming the same diet, the opposite occurred as their GFR dropped while on the high protein diet from a normal range of $54-112 \mathrm{ml} / \mathrm{min}$. to a potentially dangerous $32-91 \mathrm{ml} / \mathrm{min}$. (Walrand et al. 2008). These results demonstrate protein's possible role in increasing GFR in a younger population while lowering it in the older. It appears that the older subjects' kidneys responded much differently to a high protein load. In the same study, researchers found that high protein intake $(3.0 \mathrm{~g} / \mathrm{kg}$ fatfree mass) for ten days increased net daily nitrogen balance in both the young and elderly but had no beneficial effects on muscle protein synthesis and mitochondrial function. Thus, researchers do not support the theory that older people already consuming adequate amounts of protein may benefit from very high protein intakes (Walrand et al. 2008). The question remains, does protein contribute to renal disease through its effects on calcium excretion and changes in GFR? And, are the kidneys of older adults adequate when it comes to handling large amounts nitrogen from proteins? Longer-term studies are needed to accurately weigh the risks and clarify evidence surrounding this issue. 
There is research supporting both sides of the issue when it comes to discussing increasing protein requirements with age. Physiologically we know there are changes and problems that may be somewhat alleviated with increases in protein intake. However, the fact that no Tolerable Upper Level has been set does not completely assuage the fear of its potential for increasing calcium excretion and subsequently damaging bone and kidney function. In a recent review of the literature surrounding the issue of increasing protein requirements in older adults, Gaffney-Stomberg and colleagues conclude that a protein intake of 1.0 to $1.3 \mathrm{~g} / \mathrm{kg} /$ day is needed to offset the typically lower energy intake and impaired insulin response in the elderly (GaffneyStomberg et al. 2009). In another published paper on the topic, Wolfe concludes that a protein intake of 1.6 to $1.8 \mathrm{~g} / \mathrm{kg} /$ day may be warranted to support anabolism at rest or after exercise in older men and women (Wolfe 2006). Given the recent available data, a compromise that involves increasing the protein RDA for older adults to $1.0-1.2 \mathrm{~g} / \mathrm{kg} / \mathrm{day}$ (or approximately $13-16 \%$ of total energy) would maintain normal calcium metabolism and nitrogen balance without negatively affecting renal function (Gaffney-Stomberg et al. 2009). However, more research is needed to continue looking at the potential benefits and consequences of a higher protein diet when considering all realms of health in an elderly population.

\subsection{Physical Measures of Health and Functioning in Older Adults}

Aside from endogenous, clinical measures of health, monitoring changes in body composition and physical functioning across the lifespan provide researchers with valuable information about the aging process and effective prevention/intervention strategies for maintaining the best quality of life possible for older adults. 


\subsubsection{Body Composition Changes with Age-Physiological and Financial Impact}

Body composition, a person's mass as a function of relative amounts of bone, muscle and fat tissues, does not remain constant throughout the lifecycle. A person's fat-free mass begins to progressively decrease after 20 years of age to total a near $40 \%$ loss between then and the age of 70 whereas a person's fat mass, as a relative percentage of tissue, tends to increase steadily (Villareal et al. 2005). In addition to shifts in amounts of various body tissues, older adults generally experience a redistribution of both body fat and muscle. As mentioned previously, adipose tissue in older adults typically begins to shift from a subcutaneous location to intra-abdominal and intra-muscular storage while central fat-free mass decreases relative to total peripheral fat-free mass (Villareal et al. 2005). Thus, it is common for older adults to display larger abdominal (core) areas with while maintaining relatively small limbs.

\subsubsection{Sarcopenia Defined and Mechanisms Behind the Condition}

Sarcopenia, Greek for "poverty of the flesh," describes an age-associated loss of muscle or lean body mass and an associated loss of strength and function. Put broadly, the term encompasses "age-related changes that occur within skeletal muscle and encompasses the effects of altered central and peripheral nervous system enervation, altered hormonal status, inflammatory effects, and altered caloric and protein intake" (Doherty 2003). In terms of a hard numbers diagnosis, researchers define the condition as a total appendicular skeletal muscle mass $\left[(\mathrm{kg})\right.$ per height $\left.\left(\mathrm{m}^{2}\right)\right]$ of more than two standard deviations below that of a young, healthy reference population (Doherty 2003, Cruz-Jentoft et al. 2010). In the literature, reference population values are commonly 
taken from the Rosetta Study in which researchers used dual x-ray absorptiometry (DXA) to measure the body composition of a healthy large cohort of young adults aged 18 to 40 years (Gallagher et al. 1997).

Using this numerical cut-off for the classification of sarcopenia, researchers in the New Mexico Elder Health Survey used a DXA scan to estimate appendicular skeletal muscle mass in 883 randomly-selected older adults to quantify the proportion of a population affected by this condition at various life stages. Researchers concluded that the prevalence of sarcopenia increased from 13 to $24 \%$ from 65 to 70 years of age and then jumped to $50 \%$ of the population in subjects 80 years of age and older (Baumgartner et al. 1998).

In a recently published report from the European Working Group on Sarcopenia in Older People (EWGSOP), experts reached a consensus and developed a practical clinical definition and diagnostic criteria for age-related sarcopenia. The group recommended using the presence of both low muscle mass and low muscle function (strength or performance) to diagnosis sarcopenia because muscle strength does not depend solely on muscle mass and the relationship between the two is not necessarily linear (Cruz-Jentoft et al. 2010).

The EWGSOP recommends the use of the following gold standards for assessing muscle mass: CT scans, MRI and DXA. The group recommended handgrip strength and knee flexion/extension tests as good measures of muscular strength and power and usual gait speed, timed up-and-go walk/dynamic balance and stair-climb power tests to assess functional performance. The EWGSOP's algorithm for the diagnosis of sarcopenia is shown in Figure 2.1 below. 


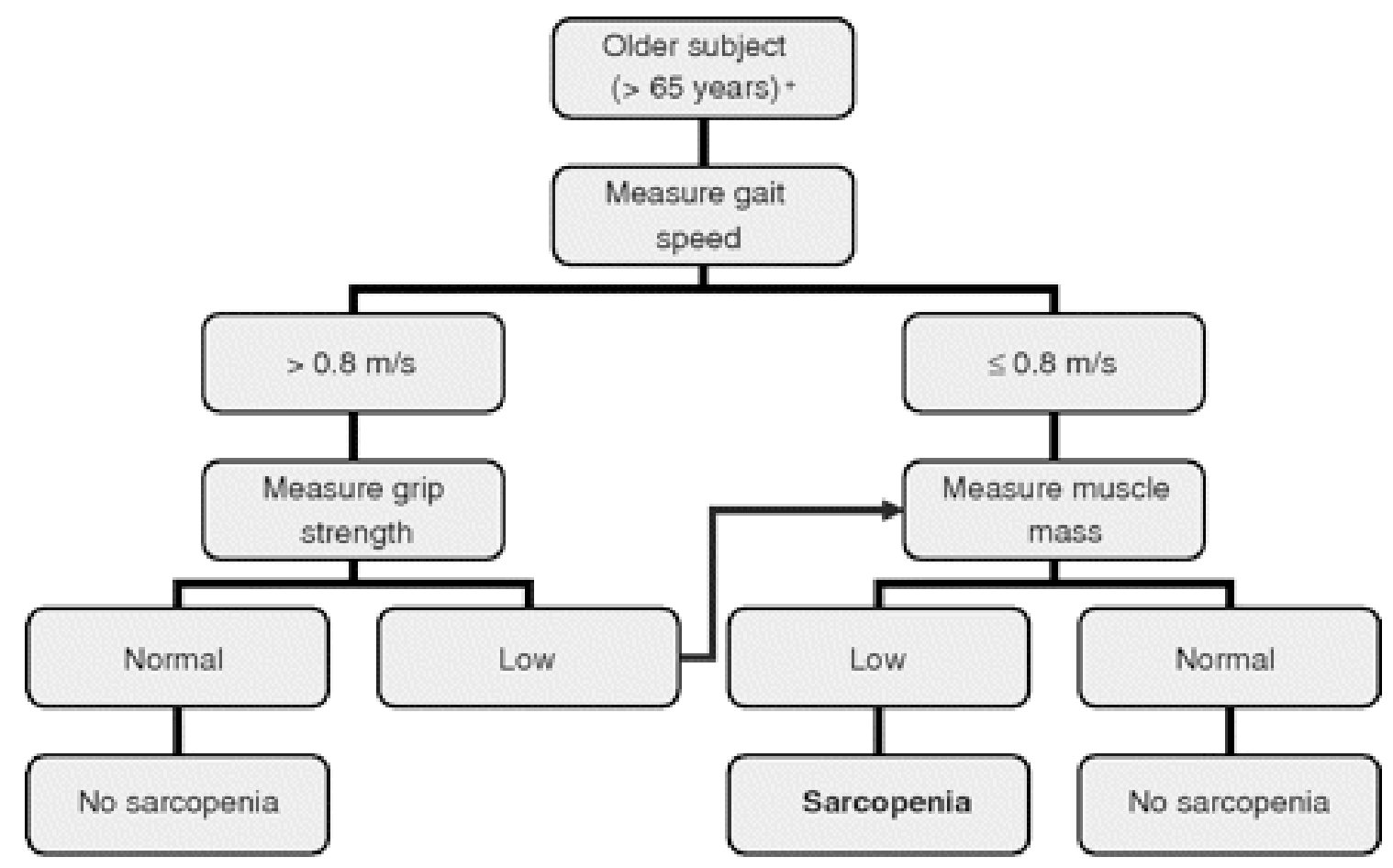

Figure 2.1 EWGSOP-Suggested Algorithm for Sarcopenia Case Finding in Older Individuals

Taken from Cruz-Jentoft et al. 2010

All older adults experience muscle loss to some degree, even elite masters' athletes.

Whether or not this becomes a notable, clinically evident problem depends upon a number of interrelated factors. With aging comes a general withdrawal in many muscle anabolic stimuli and possibly an increase in lean tissue catabolic factors (Roubenoff \& Hughes 2000). The central nervous system has been shown to slow in function as is evidenced by a loss of alpha-motor neuron input to muscle. Muscle fiber enervation is a crucial factor to the maintenance of muscle mass and strength, so this falling off of motor neuron signaling may lie at the heart of the condition. Reductions in growth hormone, estrogen, and testosterone may also play a role. Sex steroids have important anabolic effects on muscle and have also been shown to possibly inhibit the production of 
inflammatory, catabolic cytokines such as interleukins1 and 6 (Roubenoff \& Hughes 2000).

Increased intra-muscular and intra-hepatic fat present in older adults often results in increased insulin resistance and a decrease in the body's ability to utilize dietary fuel for energy and protein anabolism. Possibly even more important is insulin's role in slowing muscle catabolism and preserving lean tissue. In a study by Volpi and colleagues comparing the response of muscle protein synthesis, breakdown and net balance to ingestion of an amino acid-glucose mixture in both young and old adults, researchers concluded that the prime factor responsible for the impairment of muscle protein synthesis in the older group during intake of an amino acid-glucose mixture was an alteration in the muscle protein synthesis response to induced hyperinsulinemia. In other words, the aging muscle was unable to effectively respond to the ingested glucose and, therefore, was not able to produce a "normal" anabolic response, most likely due to insulin insensitivity of the aged muscle (Volpi et.al. 2000). In a similar study conducted on rats, analyzing the anabolic effects of insulin and amino acids independently and combined, researchers found that, regardless of age, insulin is required in addition to amino acids for muscle protein synthesis to occur. However, the response of muscle protein synthesis to dietary amino acids alone seemed to be blunted in older rats (Prod'homme et al. 2005).

Other proposed factors contributing to sarcopenia include insufficient protein and energy intake and a lack of physical activity. Elderly persons who exercise less experience greater losses in strength and lean mass than do their more active peers, but the question remains as to whether loss of strength and lean mass play a role as a cause or 
an effect in this situation. Perhaps those older adults with less lean mass to begin with are naturally unable to remain as physically active as those with a greater starting size and strength. Regardless, exercise is one of the most controllable countermeasures against sarcopenia, as even frail nursing home patients have shown some improvement in amounts of lean muscle mass in response to training (Roubenoff \& Hughes 2000).

Figure 2.2 demonstrates the interrelated mechanisms behind sarcopenia and the ambiguity in establishing cause and effect relationships for this condition.

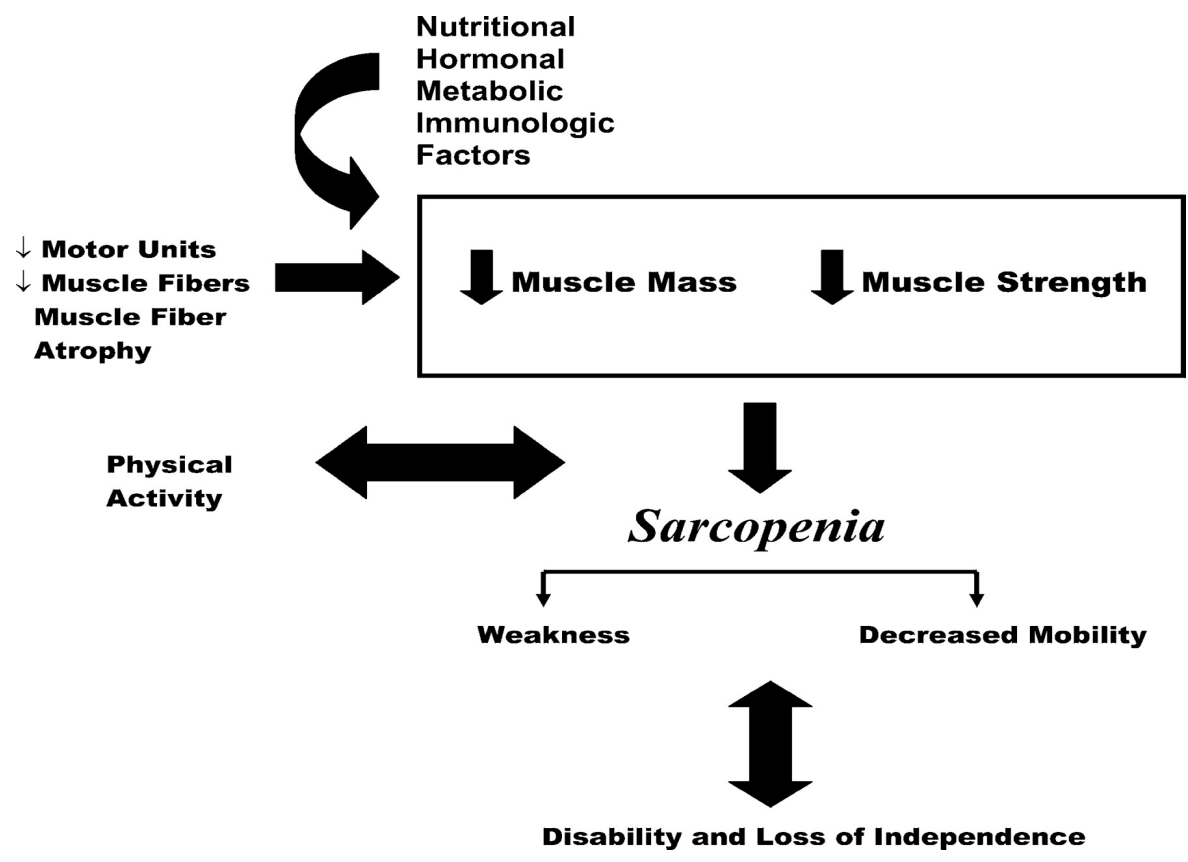

Figure 2.2 Interrelated Mechanisms of Sarcopenia A number of nutritional, hormonal, metabolic and immunological factors contribute to decreases in muscle mass and strength as witnessed by a decrease in alpha motor units and muscle fibers. These symptoms partially define sarcopenia as a condition. Sarcopenia may contribute to a person's ability to be physically active and/or a lack of physical activity may increase likelihood for developing sarcopenic symptoms. Sarcopenia results in whole-body weakness, a lack of mobility and decreased independence. The two-way arrow at the bottom of the figure reflects the notion that physical disability may also function as a cause of decreased mobility and weakness.

Taken from Doherty, T. J. J (2003) 


\subsubsection{Functional and Health Implications of Reduced Lean Body Mass}

The physical effects of sarcopenia have major implications for the health, wellbeing and independence of older adults. The New Mexico Elder Health Survey, a large, population-based cross-sectional study of elderly men and women in New Mexico, found that sarcopenic women, defined as having an appendicular skeletal mass at least two standard deviations below that of healthy young adults in the Rosetta study, had 3.6 times higher rates of disability when compared with non-sarcopenic older women; sarcopenic men had 4.1 times the rate of disability (Doherty 2003).

Loss of muscle and muscle cross-sectional area directly affects strength and a person's ability to easily and safely perform everyday tasks. In a cross-sectional study of 30 community-dwelling frail elderly women, researchers found that muscular strength was strongly related to fat-free mass as measured via bioelectrical impedance analysis techniques (to be discussed in later sections). In this study, strength was assumed to be predictive of everyday functional ability and was measured using a health survey questionnaire, grip strength testing, an up-and-go walk test and isometric muscle strength testing of the elbow and knee flexors. Fat-free mass was significantly and positively correlated with all measures of strength except for the timed up and go, a test of gait speed, coordination and reaction time. It was also found that the women's perceived functional capacity, as measured with the health questionnaire, was positively correlated with fat-free mass and measured strength (Payette et al. 1998).

In another cross-sectional study analyzing data from 14,818 people examined as part of NHANES III (1988-1994), researchers found similar results. In this study, Class I sarcopenia was considered present in subjects with a Skeletal Muscle Mass Index 
[(Skeletal Muscle Mass/Total Body Mass) x 100] within one to two standard deviations of young adult values. Class II sarcopenia was considered present in subjects with a Skeletal Muscle Mass Index below two standard deviations of young adult values. After controlling for BMI, age, race/ethnicity, smoking, alcohol consumption, physical activity and presence of chronic disease, researchers found that older men and women with Class I and Class II sarcopenia had greater odds of experiencing difficulty performing activities of daily living (stooping, balancing, climbing stairs, lifting and carrying ten pounds.) (Jannsen et al. 2002). Thus it appears that maintenance of lean body mass plays an important role in maintaining quality of life in the aging population.

A large longitudinal study by Francesco and colleagues resulted in similar findings to both Payette and Jannsen. This five year investigation of 274 men and women (ages 68 to 78) provided evidence of an association between worsening disability and a decline in appendicular and fat-free leg mass. Subjects in the study were asked to identify disability based on their ability to carry out basic activities of daily living (bathing, getting out of bed, dressing, and eating), instrumental activities of daily living (shopping, using the phone, cooking, and other housework) and their ability to walk for extended periods, including up and down stairs. At the 5.5-year follow-up date, a significant decline in total, appendicular, and leg fat-free mass was observed in both genders, independent of weight change, and the probability of having a worsening disability was significantly associated to the decline in fat-free mass; moreover, the risk increased two-fold in the group of participants losing leg fat-free mass (Zoico et al. 2004).

Along with a decrease in the ability to perform activities of daily living, decreased muscle mass has been shown to correlate directly with increased mortality. Losses in 
strength increase susceptibility to falls and accidents and decreased muscle mass serves as both a consequence and an indicator of declining physical health. In another prospective study by Wannamethee and colleagues, a group of 4107 older men (60-79 years) from the British Regional Heart Study were followed over a 20-year period to assess physical measurements, body composition and all-cause mortality. Subjects with diagnosed heart failure were excluded from the study due to their exceptionally high mortality rates. Researchers concluded that central adiposity and decreased muscle mass, as measured by waist circumference and mid-arm muscle circumference respectively, were significantly associated with increased mortality. Furthermore, researchers concluded that body composition assessments proved better predictors of mortality than simple body weight measures alone (Wannamethee et al. 2007).

\subsubsection{Financial Impact of Sarcopenia}

Aside from individual health risks associated with sarcopenia, this condition places a significant burden on the U.S. economy, reflected by an increased spending on healthcare. Janssen and colleagues attempt to quantify the direct healthcare costs of sarcopenia in the United States based on this condition's effect on increased physical disability risk in older persons (ages 60 and over). Researchers used data from the U.S. Census, NHANES III and the National Medical Care and Utilization Expenditure Survey to estimate the predominant direct costs of the disease: Hospital care, outpatient procedures and home healthcare expenditures. Indirect costs such as lost productivity were not taken into account. Based on these analyses, the estimated direct healthcare cost attributable to sarcopenia in the United States in the year 2000 was $\$ 18.5$ billion $(\$ 10.8$ billion from men and $\$ 7.7$ billion from women). These numbers break down to an 
additional nine hundred dollars per sarcopenic person per year. Notable also is that estimated yearly direct costs of osteoporotic fractures in the United States is $\$ 16.3$ billion, making sarcopenia an even more problematic yet far less recognized problem in our society (Janssen et al. 2004).

\subsubsection{Methods for Estimating Body Composition}

The overwhelming prevalence and cost of sarcopenia, both physically and financially, highlight the need for effective methods for diagnosing changes and losses in lean body mass. Currently, there are a wide variety of methods used to assess body composition in individuals, each with its own strengths and weaknesses when used in the assessment of older adults. The most commonly used techniques include: anthropometric measures, bioelectrical impedance analysis, densitometry techniques, and dual x-ray absorptiometry.

\subsection{Anthropometry}

Anthropometric measures such as height, weight, skinfold and girth measurements provide quick, easy, noninvasive and inexpensive methods of assessing the health status of individuals within a population. Taking such measurements in an older population does provide valuable information but, due to physiological changes, functional limitations and a lack of relevant reference data, these methods carry with them a major set of challenges.

When measuring an elderly individual's height and weight and using these to calculate body mass index and predict health outcomes, problems stem from the potential functional limitations of obtaining accurate standing measurements as well as changes in 
body composition that are not always reflected in weight changes. Due to osteoporosis, vertebral disc compression and other joint and spine conditions, it is common for older adults to experience a loss of height with age close to $1 \mathrm{~cm}$ every ten years after age 40 and an even more rapid loss after 70 years of age (NIH 2009). In one longitudinal study including over 1,000 elderly Swedish individuals, researchers found that, over a 25-year period, the mean height for males decreased by $4.0 \mathrm{~cm}$ while that for females decreased by $4.9 \mathrm{~cm}$ (Dey et al. 1999).

To remediate measurement problems associated with height loss and functional challenges, alternate tools including equations using arm span and lower and upper leg lengths to estimate height and weight are useful in an elderly population. Researchers in the National Health Examination Survey (NHES) and NHANES III found that knee height and age were the most useful predictors for estimating stature in subjects with functional disabilities or excessive spine curvature (Chumlea \& Guo 1992; Chumlea $e t$ al.1998). Other studies use self-reported height and weight measures in which researchers may ask what the subject's height was as a young adult. However, it has been documented that self-reported values for height and weight tend to be less accurate and reliable as a person ages (Kuczmarski et al. 2001; Lawlor et al. 2002).

Weight in an older adult does not decrease as predictably and consistently as height but with the right equipment, can be measured much more accurately, even in a person with a functional disability. While it is common for older adults to lose weight and grow frail over time, overweight and obesity remain prevalent health problems for every age group. To assess both under- and overweight in a population, measures of height and weight are used to determine a more practical, useful number: body mass index. 
A person's body mass index (BMI) defined as (Weight $(\mathrm{kg}) /$ Height $\left.(\mathrm{m})^{2}\right)$ is a quick and easy measure for classifying healthy weight within a population. BMI is used to classify individuals as underweight, healthy, overweight or obese, to identify individuals at risk for developing obesity-related diseases and to monitor changes in body composition (U.S. Dept. HHS 2000; WHO 1998). In clinical nutrition applications, a person's weight and BMI can also be useful in assessing dietary nutrient adequacies. The range of BMI values used to classify adults is presented in Table 2.1 below:

Table 2.1 Adult BMI Classifications

Classification BMI Value

$\begin{array}{ll}\text { Underweight } & <18.5 \\ \text { Normal Weight } & 18.5-24.9 \\ & * 22.0-27.0 \\ & 25.0-29.9 \\ \text { Overweight } & >30.0 \\ \text { Obese } & (30.0-34.9,35.0-39.9, \geq 40) \\ \text { (Class I, II, III) } & \end{array}$

* Alternate "normal" range for older adults WHO Report (1998)

Note that in Table 2.1 the suggested "normal" BMI range for older adults (22.0-27.0) lies above that of younger adults (18.5-24.9). The discrepancy is due to a significant increase in mortality at lower BMIs that is often observed in the elderly, possibly due to its reflection of low lean body mass (Seidell \& Visscher 2000). There is still some controversy regarding the need for a separate healthy BMI range for older adults but generally researchers have not seen the same associations between high BMI and 
mortality as those witnessed in younger adults (Seidell \& Visscher 2000; Corrada et al. 2006). Rather, studies have commonly found that low BMI and unintentional weight loss is often a greater mortality threat to older adults than obesity (Locher et al. 2007).

In one analysis of data from nearly 5,000 older men and women enrolled in the Cardiovascular Health Study, researchers were able to conclude that the risk of developing negative weight-related health outcomes (myocardial infarction, stroke, sleep apnea, urinary incontinence, cancer, osteoporosis) was not different between "healthy" and "overweight" older adults, classified as a BMI of 18.5-24.9 and 25.0-29.9 respectively. The risk for developing physical disability and arthritis was modestly increased in the overweight group while the risk for the development of Type 2 diabetes increased in this group by $78 \%$. However, after adjustment for all relevant covariates, the overall mortality rate was $11 \%$ lower in the overweight group, thereby supporting the theory that a healthy BMI cutoff point may be greater than 25.0 in older adults (Janssen 2007).

On the other hand, an extremely high body mass index in this age group may not necessarily result in decreased disease incidence or an improved quality of life. A report put out by the Centers for Disease Control summarizes NHANES physical functioning data in relation to BMI among 5,000 subjects 60 years of age and older. The study found that those people in the highest BMI range (>32.4) reported more difficulties performing nearly all functional activities (mobility, strength, endurance and social activities) compared with those in the mid-range (BMI 22.3-32.4). In addition, the lowest BMI category $(<22.3)$ reported few significant differences from the mid-range group. The only significantly lower functional scores in the low BMI group when compared to the 
mid-range group were in managing money, carrying ten pounds, doing household chores, preparing meals and grasping small objects (Ervin 2006). Therefore, it appears that those subjects with a BMI between 22.0 and 32.0 maintained the greatest quality of life.

As is evidenced by the literature, data regarding a healthy BMI range for older adults remains inconclusive but most studies agree that elderly adults with a lower BMI experience increased rates of mortality. A better application of BMI in the elderly includes watching for changes within an individual over time. A sudden decrease in BMI may indicate a progressive disease state or a sudden reduction in energy intake.

However, due to tissue changes such as sarcopenic obesity and muscle wasting that BMI values are unable to directly reflect, it is also important for researchers to consider more direct measures of body composition.

Circumferences and skinfold measures, both anthropometric assessment tools as well, provide additional information regarding body composition. While these measurements cannot provide differential changes in fat and lean mass, they do provide estimates of total body composition through their usefulness in prediction equations and in measuring individual changes over time.

Circumferences are affected by fat mass, muscle mass and skeletal size and, for clinical purposes, are commonly measured at the waist, abdomen, hip, thigh, biceps, and calf (Heyward 2006). In the elderly, waist circumference is found to be a strong predictor of body fatness, possibly even more so than body mass index, as it provides a sensitive detection of fat shifts from the limbs to the core that occur in an aging body (Visscher et al. 2001). Waist circumference has been shown to be a strong predictor of morbidity and disability in old age (Ramsay et al. 2006). Calf circumferences also 
provide useful information, specifically as a measure of malnutrition in aging. Researchers have found calf circumference measures to be strongly correlated with skinfold thickness, BMI, fat-free mass and serum albumin. Thus, this measure has potential as a useful tool in estimating body composition (Bonnefoy et al. 2002).

In one 10-year longitudinal study of 129 elderly subjects, researchers found that each circumference taken (mid-arm, abdominal, hip, mid-thigh and calf) was significantly associated with total-body fat mass and percent body fat at baseline and follow-up measurements in both men and women, with changes in waist and hip circumferences explaining most of the variance in fat mass change (Roubenoff \& Hughes 2000). These same researchers suggest further investigation into changes in the waist to thigh ratio, as this would measure both the increase in abdominal fat and serve as a predictive indicator of muscle loss or sarcopenia, as reflected by decreased thigh girth (Roubenoff \& Hughes 2000).

As simple and inexpensive as they are, when compared to other tools for measuring body composition, to be discussed in following sections, researchers argue that circumferences alone may not as accurately reflect the distributions of whole-body fat and lean mass. When measured against hydrostatic weighing, DXA and prediction equations including total body mineral mass, body water and body density, anthropometric measures were found to be associated with unacceptably high error rates (Chumlea \& Guo 1992).

Skinfold thickness measurements provide anthropometric values, commonly used in conjunction with circumference measures, to estimate total body fat. Measurements are routinely performed on the right side of the body and are taken at some or all of the 
following regions: chest, subscapular, suprailiac, abdominal, midaxillary, triceps, biceps, thigh and calf (Heyward 2006). Researchers may choose to use a three- or seven-site method with gender-specific fold locations chosen. When used in an elderly population however, these measures may not always reflect changes in total body fat mass. As mentioned previously, fat redistribution with age includes shifts from subcutaneous to intra-muscular and intra-abdominal storage. Thus, subcutaneous fat mass may not be as predictive of total body fat mass in older adults as it is in the younger (Roubenoff \& Hughes 2000; Gause-Nilsson \& Dey 2005). Furthermore, skin elasticity begins to decrease due to changes in collagen content while atrophy of subcutaneous adipocytes leads to increased tissue compression. For these reasons, skinfold measures tend to underestimate total body fat mass in an elderly population (Bales \& Ritchie 2009, GauseNillson \& Dey 2005).

In addition to physiological problems with skinfold analysis in the elderly, the precision and training required of technicians leaves room for high rates of inter- and intra-person measurement error (Heyward 2006). Population and age-specific prediction equations also play a role in the accuracy of these measurements. There have been very few published prediction equations in which elderly subjects have been used, even though it is believed that equations tested on younger adults may not provide reliable estimations for older adults (Gause-Nillson \& Dey 2005). For both circumferences and skinfolds, there is a great need for reliable reference data to develop accurate prediction equations. 


\subsection{Bioelectrical Impedance Analysis}

Bioelectrical impedance analysis (BIA) is another noninvasive, relatively easy field method for the evaluation body composition. In this method, a low-level electrical current of $50 \mathrm{kHz}$ is passed through the body, generally via electrodes attached to a subject's wrist and foot, and the impedance or opposition to flow is measured. Other BIA analyzers measure resistance to flow in only the lower or upper body. As with anthropometric measures, BIA electrical impedance numbers (Ohms) are used in population-specific prediction equations to estimate total body fat (Heyward 2006). This method is based on the principle that tissues with more water and electrolytes conduct the current more readily than those with less. Thus the electrical current passes more quickly through hydrated muscle than through adipose tissue, which contains relatively little water. Along with estimates of total body fat and lean tissue, BIA methods provide an estimate of total body water. Prediction equations for using BIA measurements are based on gender, age, height, weight, ethnicity and physical activity level but, as is true for anthropometric measures, equations developed on and for the elderly remain sparse and largely invalidated (Heyward 2006).

Like any estimate of body composition, BIA methods have their shortcomings. One major source of error with this technique is the intra-individual factors that affect hydration status. Dehydration and fluid loss due to exercise tend to increase resistance to flow thereby overestimating body fat. Conversely, fluid consumption and an increase in blood flow and skin temperature due to activity lead to less resistance to flow and potential underestimates of total body fat. Bioelectrical impedance techniques also assume that the body is shaped like a perfect cylinder with uniform length and cross- 
sectional area. This assumption, of course, is not entirely true and may further reduce the accuracy of this technique (Heyward 2006). It is vital for a researcher to be properly trained and to inform the client about pre-testing guidelines to control for factors that could account for measurement error.

In the elderly, the validity of BIA techniques have only been established using small samples with limited age groups; reference data for adults over the age of 80 is particularly sparse. With the right age-specific, cross-validated prediction equations, BIA has the potential to serve as a reliable tool but because of tissue and body water changes with age, current equations based largely on younger adults lack accuracy in their predictive abilities (Svendsen et al. 1991). It is also important to note that, while the test is relatively easy to administer in older adults, it cannot be used reliably on those taking diuretics or subjects with pacemakers or prosthetics, all possibilities in an elderly population (Heyward 2006).

\subsection{Densitometry}

Two main densitometry techniques for estimating body composition in older adults are hydrostatic weighing and air displacement plethysmography, also known as "The Bod Pod." Both methods calculate body volume through measurement of either water or air displacement when the body is submerged or encased in the respective medium. Body density is then estimated from the ratio of body mass to body volume (Heyward 2006). Like every other body composition estimate mentioned previously, densitometry also relies on prediction equations and is therefore an indirect estimate of body composition.

Hydrostatic weighing is a widely used technique in which a subject is completely submerged in a water tank where their underwater weight is taken upon maximal 
expiration. This method is based on the principle that weight loss under water is directly proportional to the volume of water displaced by the body's volume (Heyward 2006). Body volume is subsequently corrected for residual volume left in the lungs and the volume of air in the gastrointestinal tract (assumed to be 100ml). Hydrostatic weighing is a two-compartment model, meaning it divides the body into only two constituents-fat and fat-free mass. It relies on assumptions of constant densities for each tissue of $0.0900 \mathrm{~kg} / \mathrm{liter}$ for fat and $1.1000 \mathrm{~kg} / \mathrm{liter}$ for fat-free components and assumes that the relative amounts of the aqueous, mineral and protein components of fat-free mass are known and constant in all individuals. However, tissue density and tissue composition changes later in life may decrease the accuracy of these constants (Clasey et al. 1999).

Other obvious problems with this method when employed in an older population stem from the lack of age-specific reference data and the fact that many older adults are unable to physically assume the position and perform the procedure necessary to obtain accurate measurements. While there are prediction equations that can be used for an individual reluctant to submerge his or her face or for those individuals unable to fully expire the air in their lungs, the physical demand of this assessment makes hydrostatic weighing a technique not commonly used in older adults (Heyward 2006).

Air displacement plethysmography (ADP) or "The Bod Pod" relies on many of the same principles as hydrostatic weighing but is generally a much more comfortable and less physically demanding procedure. Rather than measuring body volume via water displacement, a subject enters a pressurized chamber and sits quietly while the amount of air displaced by his or her body is measured and entered into a body composition prediction equation (Heyward 2006). 
Similar to hydrostatic weighing, problems with ADP include the inability of prediction equations validated in younger adults to accurately predict the body composition of an older population. Again, changes in tissue hydration and the composition of fat-free tissue as a person ages warrants the need for age-based prediction equations with age-specific tissue density constants. Physically, the ADP method may be more feasible for older adults but it is still not as widely used and accepted as the current gold standard for body composition estimation—Dual x-ray absorptiometry.

\subsection{Dual X-ray Absorptiometry (DXA)}

Dual x-ray absorptiometry (DXA) is considered by many as the current "gold standard" for body composition assessment and is quickly gaining recognition as a reference method for body composition research. This three-compartment method involves the estimate of bone mineral, fat and lean soft tissue mass through the use of $x$ ray beams that differentiate between tissue types via the unique penetration of each. This method requires minimal subject cooperation and technician skill and is therefore useful in nearly all populations and clinical settings. It merely requires a 3-4 minute whole body scan of the subject, while lying quietly in a supine position. The dose of radiation from each $\mathrm{x}$-ray beam remains very low and is safe in all populations aside from pregnant women (Heyward 2006).

DXA is a unique method in that it can provide regional values for body composition rather than just a single value for total body fat. Unlike methods mentioned previously, DXA stands as a more direct measurement of tissues and can provide the subject and technician with a visual image of lean and fat distribution (Rutledge 2007). Scans are based on the underlying assumption that all non-fat and non-bone tissue is muscle. This 
assumption is likely more valid at the regions between the joints such as the mid-thigh and calf where the amount of tendons and cartilage is small. Typically, DXA scans are taken at many subregions along the body to increase the chance of overall accuracy in estimation (Proctor et al. 1999).

It is important also to note though that skin is included as part of the DXA fatfree/muscle mass estimates where it is not in other methods of body composition estimation. Therefore, there is some criticism that lean mass estimates may tend to run higher when this method is used (Visser et al. 1999). Another study suggested that DXA commonly produces higher values for fat-free mass due to its assumption of a constant hydration of the skeletal muscle compartment across individuals. Therefore, increases in intramuscular fat deposition or increased muscle hydration due to edema, both resultant of obesity and/or aging, may be falsely detected by DXA as an increase in lean tissue (Proctor et al. 1999).

While DXA techniques do have their own set of drawbacks, there have been many validation studies that have upheld the accuracy and precision of estimates obtained through this method. Due to the minimal skill required of technicians and the small amount of intra-individual variation that comes into play in other methods of body composition estimation, DXA scans have very good reported reproducibility and therefore have been shown to be accurate predictors of short and long-term changes in body composition, even in older adults (Visser et al. 2003).

In one cross-sectional study of 60 healthy elderly individuals of varying BMIs, absolute differences in estimation of fat mass between DXA and a four-compartment model (body density by hydrostatic weighing, total body water by deuterium dilution, 
total body bone mineral mass by DXA, and body weight) were relatively small, with differences of less than $1 \mathrm{~kg}$ (Salamone et al. 2000). This finding is significant, as the four-compartment model has been used as a reference model for accurate body composition estimation due to its use of multiple, different estimation techniques (Clasey et al. 1999). In the same study, DXA leg slice scans were also found to be moderately to highly correlated with computed tomography (CT) scans at all four leg sites (p-value $<0.0001)$. CT scans serve as another criterion method for assessing body fat; they have been shown to accurately assess amounts of adipose tissue and are oftentimes used in comparisons with DXA scans of fat tissue by multiplying their value by .80 (assumption that $80 \%$ of adipose tissue is fat). Based on findings from this cross-sectional validation study, researchers concluded that DXA measurements of total body fat and leg fat mass provide accurate and reliable estimates in elderly men and women (Salamone et al. 2000).

Perhaps even more important in older adults is the ability of DXA scans to directly measure lean tissue mass. In one cross-sectional validation study of fan-beam dualenergy X-ray absorptiometry for measuring total body fat-free mass and leg muscle mass, researchers compared DXA measurements at four leg regions with two criterion methods - a four-compartment body composition model and multi-slice computer tomography of the legs, both described previously. Researchers defined total body fatfree mass as all lean soft tissue plus total body bone mineral content. Subjects in the study included healthy older men and women, 70-79 years of age with a wide range of BMIs. Upon comparison of these different techniques researchers found very good agreement between DXA measures of muscle mass when compared with the criterion 
methods, with $\mathrm{R}^{2}$ values greater than 0.95 when comparing DXA against the fourcompartment model and DXA against CT values for leg sub-regions and total leg muscle mass. Thus, these findings suggest that the fan-beam DXA procedure offers considerable promise for the assessment of fat-free body mass and leg muscle mass among elderly subjects (Visser et al. 1999).

\subsubsection{Muscular Strength, Endurance and Quality and their Implications in Older Adults}

In addition to estimates of body composition, functional measures of health serve as important indicators of individual wellbeing and are particularly interesting when used in the assessment of older adults. As was mentioned previously, physiological changes associated with aging occur at both the cellular and whole muscle level and are associated with not only changes in lean tissue but also changes in muscular strength, endurance and quality.

\subsubsection{Muscular Strength and Endurance}

Muscular strength, or the amount of force available for one maximal muscular contraction, is often used as an indicator of functional mobility and health in the elderly. Similar to strength, muscular endurance involves the ability of a muscle to perform work repeatedly over time and is highly indicative of functional health. Possessing normal or high levels of muscular strength and endurance provide benefits to the older adult in their enhancement of a person's ability to perform activities of daily living (ADLs) such as standing from a chair, lifting a bag of groceries, playing with grandchildren and avoiding sudden falls. 
Muscular strength and endurance are commonly measured through the use of dynamometers and functional mobility testing. NHANES 2001-2002 used knee strength dynamometer testing, timed walk tests and Romberg balance testing to assess strength and endurance. In subsequent years (2003-present), NHANES researchers chose to abandon direct testing in lieu of physical activity monitors and a self-reported physical functioning questionnaire (CDC 2009).

Knee strength dynamometer testing is a commonly used tool for the assessment of lower body dynamic strength and as a predictor of functional mobility as it measures both eccentric and concentric contractile ability of the muscles at a range of constant velocities. The ability to perform a dynamic task such as walking or regaining one's balance upon stumbling depends upon both torque production and speed of contraction in the muscle, both of which are measured by this type of testing. The knee extensors, or quadriceps muscles, are commonly used in the testing of older adults due to the fact that they contain a mix of both Type 1 and Type 2 muscle fibers and have been shown to decrease in size, strength, and usage as individuals discontinue high power activities such as jumping and running as they age (Lanza et al. 2003).

It is widely accepted that both concentric and eccentric contractions of the knee extensor muscles, as measured by dynamometer testing, appear to decrease in force and power at all velocities as individuals age (Lanza et al. 2003; Lindle et al. 1997). In one study measuring knee extensor strength in a large sample of people from the Baltimore Longitudinal Study on Aging, researchers concluded that concentric strength decline begins in the fourth decade of life for both men and women and proceeds to decline at a rate of $8-10 \%$ per decade thereafter. While losses in concentric and eccentric strength 
with age were measured as similar, researchers from this study concluded that eccentric strength loss in women might start a decade later than their concentric strength loss (Lindle et al. 1997).

In the very large-scale Health, Aging and Body Composition Study, researchers used isokinetic strength testing to assess strength of the knee extensors as measured by maximal torque production over the entire range of motion of a leg extensor exercise. Researchers also tested whether a maximal voluntary effort during this type of testing would represent the true contractile ability of the muscle. To do this, technicians superimposed electrical stimulation of the muscle on top of maximal voluntary contraction only to find no additional torque production in the quadriceps of men and women. Thus, maximal voluntary effort of the knee extensors was suggested as a valid measure of strength in the elderly (Goodpaster et al. 2001).

Grip strength testing, assessed as the maximum force attained by a one-time hand squeeze, is easily measured with a handgrip dynamometer and has been proven useful in predicting future disability and current whole body strength in older adults (Rantanen et al. 1999). In one large longitudinal study of 3200 adults (average age of 54 years), handgrip strength of subjects at baseline was able to accurately predict functional disability and limitations 25 years later, defined as a gait speed less than $0.4 \mathrm{~m} / \mathrm{sec}$ and an inability to rise from a chair without the use of their arms. In the same study, subjects in the lowest tertile of handgrip strength at baseline also reported greater difficulty walking, lifting and performing other activities of daily living at the 25-year follow-up visit (Rantanen et al. 1999). 
Due to the cost and time constraints of repeated testing, muscular strength tests are generally conducted only once, maximally during a single-session. There is some concern regarding this protocol due to possible subject apprehension about performing unfamiliar exercises in a lab environment and resultant inability to perform to full capacity. Some research recommends conducting practice trials to familiarize subjects with the exercise protocol before test day, thus increasing the validity of the measurements; however, findings regarding the necessity of this type of warm-up protocol are mixed (Symons et al. 2005).

In one study conducted on older, healthy men to investigate the test-retest reliability of a single session of isokinetic and isometric strength testing, researchers found a large variation in a person's score from measurement to measurement on two separate days. Thus it was recommended that, to reduce changes due to a learning effect, older adults should be required to perform more than one practice session including all exercises to be used before the actual testing day begins (Symons et al. 2005).

Conversely, Schroeder and colleagues demonstrated the opposite effect when they concluded that test-retest measures of maximal voluntary muscular strength and power testing in older men proved consistent across days, differing by at most $2.3 \%$. Thus, researchers from this study concluded that it might not be necessary to perform baseline practice tests to account for test-retest improvement (Schroeder et al. 2007). While data on this issue is mixed, it is good to at least consider the possible presence of a learning curve when any measure of strength or functional status is measured repeatedly.

Muscular strength and endurance along with balance and neuromuscular capacity are measured through the use of gait speed analysis in which time to walk a given distance is 
analyzed, oftentimes concurrently with analysis of gait biomechanics. Use of a cane or a walker is permitted with most timed walk protocols. It has been shown that, even in healthy elderly, older adults have a much slower gait velocity with a shorter step length, shorter leg swing phase and less range of motion at the hip, knee and ankle joints when compared to younger adults. Changes in not only strength, but neuromuscular function, spatial awareness and balance also account for reduced confidence in walking and hence, a reduction in gait speed (DeVita \& Hortobagyi 2000).

In a review paper discussing clinical frailty assessment in older adults, Rolland and colleagues highlight studies evaluating the usefulness of gait speed as a predictor of multi-dimensional frailty (including low muscle strength and endurance). In one study, researchers independently evaluated gait seed, grip strength and repeated chair stands and, although all had predictive accuracy for identifying changes in strength and functioning, gait speed appeared to be the best indicator, with patients walking slower than $0.65 \mathrm{~m} / \mathrm{s}$ more than 20 times more likely to be frail as those who walked faster. Additionally, gait speed served as the greatest predictor of the three in terms of predicting mortality upon six months of follow-up (Jones et al. 2004).

In addition to gait speed analysis, balance tests serve as another functional measure of postural control and stability and muscular strength and endurance. The NHANES 20032004 researchers used a modified Romberg balance test on subjects 40 years and older in which individuals were required to stand with their eyes open and closed on both compliant and non-compliant surfaces. The test was scored as either pass or fail with failure defined as stumbling or beginning to fall before 15 seconds. Other variations on standard tests of balance include standing on one leg, bending to pick something up off 
the floor and turning to look over both shoulders. In addition to subjective assessment of individuals "beginning to fall," researchers often use force platforms to measure excessive postural sway and shifts in weight (CDC 2005).

One large-scale study of 6,500 White and Hispanic elderly adults investigated lower extremity function (tests of balance, time to walk 8 feet, and time to rise from a chair 5 times) and its predictability of future disability (one to six year follow-up of ability to perform ADLs and maintenance of basic mobility). Researchers found that all tests of lower extremity function were able to accurately predict follow-up disability. When researchers looked at gait speed separate from the other tests, they concluded it to be nearly as sensitive as the complete lower extremity test battery, although not quite as precise (Guralnik et al. 2000).

One additional physical activity assessment tool that differs from direct measures of strength and endurance but is currently used by NHANES researchers is the Physical Activity Monitor (PAM). This device is a uniaxial accelerometer that attaches to a person's waist and measures duration and intensity of activity. The PAM serves as a step up from a basic pedometer in that it measures more than just walking or running and is able to decipher workout intensity. The device cannot be worn while swimming or bathing, but is useful in most other exercise situations to monitor activity levels. NHANES researchers began using the PAM and moving away from direct measures of strength and endurance in the 2003-2004 series of testing. While research concerning the use of such monitors in an elderly population is minimal, such devices prove reliable in assessing activity levels in the general adult population (CDC 2006). 
When direct functional measures are not practical to collect, researchers often use self-reported functional ability as an indirect measure of strength and endurance.

Questions commonly included in self-report assessments of function include difficulty performing basic activities of daily living (ADLs) which include walking, feeding oneself, getting out of bed and lifting and carrying, as well as more advanced instrumental activities of daily living (IADLs) such as cooking and heavy housework and advanced mobility activities such as walking or standing for extended periods of time. Several studies support the assumption that self-report is a valid measure of actual strength and functional ability.

In a cross-sectional and longitudinal study conducted by Hoeymans and colleagues, researchers followed a group of 303 elderly men for 7 years in an attempt to examine associations between self-reported and performance-based measures of functional status. Answer options for all self-report measures were dichotomous with a person either able to perform a task unassisted or not. Self-report items included three separate dimensions: ADLs, IADLs and mobility, with subjects considered disabled in a dimension if they were unable to perform one or more items in a dimension unassisted. Performance measures included standing balance, timed 8-foot walk, timed chair stand and an external shoulder rotation test. For each subject, a summary performance score was assigned as the number of tests in which the subject demonstrated "low" performance, as defined by researchers. The study was cross-sectional in that it analyzed correlations between selfreport scores and summary performance scores and longitudinal in its analysis of correlation between change in self-report versus performance scores over time (Hoeymans et al. 1996). 
Researchers in this study concluded that associations between specific performance tests and self-reported disabilities were strongest between the test for walking speed and self-reported mobility and IADLs and between external shoulder rotation and selfreported disabilities in basic ADLs. As a whole, all performance measures were at least moderately associated with self-report measures in cross-sectional analyses $(\mathrm{p}<0.05)$. Researchers also found that self-reported disabilities in ADLs were associated primarily with upper extremity impairment while self-reported disabilities in IADLs were primarily associated with impaired lower extremity function. In longitudinal analysis, researchers concluded that both self-report and performance measures at baseline predicted selfreport and performance at the three year follow-up (Hoeymans et al. 1996). This study is important in thoroughly demonstrating the validity in using self-report as a measure of strength and physical functioning in older adults.

In other published research, self-reported walking ability demonstrates an especially high predictive ability of performance-based functional capacity (Cress et al. 1995, Sayers et al. 2004, Alexander et al. 2000). In a study of 150 community-dwelling men and women aged 75-90 years, researchers found a 91\% probability that subjects would not be able to walk $1 / 4$ mile if three major questions in a walking performance interview were answered with respect to being unable to walk (Sayers et al. 2004). Similarly, Alexander and colleagues examined 221 older adults, $60+$ years, in an attempt to relate self-report to performance measures of functioning. Researchers concluded that, while all self-report scores (ADLs, IADLs and advanced mobility) were significantly related to performance measures (walking, stance maintenance and rising from a chair), self-report 
items related to walking were the strongest predictors of functional mobility (Alexander et al. 2000).

\subsubsection{Muscle Quality}

Muscle quality, or strength per unit of muscle mass, is an important concept to consider when looking at changes in older adults and dealing with conditions such as sarcopenia. Physiological changes with age include not only changes in overall mass but also in components of the skeletal muscle. As mentioned previously, loss in both number and size of type 2 fibers, decreased nerve innervation, losses in the number of protective satellite cells and increases in pro-inflammatory cytokines have all been postulated to play a role in loss of muscle quality and strength. Goodpaster and colleagues, in the Health, Aging and Body Composition Study of nearly 2,000 older adults, concluded that over the course of three years, initially healthy older men and women experienced a three times greater loss in strength than decline in muscle mass. Furthermore, maintenance or even gain of lean mass in the older adults did not necessarily prevent the loss of strength (Goodpaster et al. 2006). This study highlights the importance of not only muscle quantity but muscle quality for the maintenance of strength and proper physical functioning in older adults.

DXA scans mentioned earlier are useful in conjunction with physical strength tests for assessing whether the loss of strength with age is attributable to a loss of muscle mass or quality. Using DXA techniques, researchers can precisely measure the quantity of muscle. Combining this measure with data from strength tests such as one-repetition max tests, researchers can determine strength per unit of muscle mass (or muscle quality). Muscle biopsies are also useful in the assessment of muscle quality as they measure 
muscle atrophy, inflammation, myopathic changes, necrosis or tissue death and nerve dysfunction (Medline 2009). There is little or no risk to the patient with either of these methods, which makes both procedures very useful in nearly all populations.

\subsubsection{Frailty}

While there is a lack of consensus as to a clear, clinical definition of frailty, most researchers and health professionals can agree on major themes underlying the condition. Such themes or symptoms include: Unintentional weight loss, low muscle mass and strength, feelings of exhaustion, reduced physical activity, chronic disease or disability and changes in cognition and mood, all of which contribute to a reduced capacity to independently perform basic activities of daily living (Abellan van Kan et al. 2008; Bartali et.al. 2006). Physical frailty is resultant of the physiological changes experienced with age, all mentioned in preceding sections. Hence, measurement tools to assess the condition include all of the body composition and muscular strength and endurance tests mentioned previously. The usefulness of other assessment tools depends upon the clinical definition used and includes self-reported questionnaires regarding eating habits, activity level, and the ability to perform basic activities of daily living. The Mini Nutritional Assessment (MNA) has been largely used and validated in clinical practice to assess malnutrition or risk for malnutrition and is very useful in measuring the dietary component associated with frailty (Abellan van Kan et al. 2008).

Frailty as a condition greatly reduces a person's quality of life due to its association with loss of independence and increased risk of morbidity and mortality. While many conditions associated with frailty remain an inevitable part of aging, it is possible to delay or minimize these effects through lifestyle choices that include regular physical activity 
and healthy diet choices. Many researchers have studied and are currently investigating the prevalence of dietary protein malnutrition and the role of this macronutrient in the aging body.

\subsection{Protein Intake and its Relationship to Body Composition, Muscular Strength, Endurance and Quality, and Other Physical Measures in Older Adults}

Physiological changes experienced with aging are well established and not often welcomed by older adults. There is published research examining these changes and investigating the relationship of dietary factors. One of the major areas for investigation is protein intake in older adults and its association with lean body mass and measures of functional ability and strength. There is evidence that protein intake decreases with age and mixed evidence regarding protein's ability to preserve muscle mass and function.

\subsubsection{Current Protein Consumption Trends in the United States}

The National Health and Nutrition Examination Survey (NHANES) is the only largescale, nationally representative collection of health and nutrition data that provides extensive documentation of food intake based on within-person averages of two, 24-hour recalls. This major program of the National Center for Health Statistics, part of the Centers for Disease Control and Prevention, surveys and examines approximately 5000 people every year and produces vital health statistics for the nation entailing everything from food consumption patterns to weight and body composition data, physical fitness status and prevalence of a wide variety of diseases. Researchers commonly use data from NHANES to quantify amounts of macro and micronutrients consumed by the American 
population. It is a very useful resource in assessing protein intake across the nation (CDC 2007).

As seen from the DRI recommendations mentioned previously, protein intake is most commonly expressed in grams per day per kilogram of body weight or as a percentage of total daily energy. To assess the adequacy of populations, researchers look at a percentage of adults with intakes falling below the EAR value of $0.66 \mathrm{~g} / \mathrm{kg} / \mathrm{day}$. A key problem in assessing intake based on weight parameters lies with individuals who fall outside of a healthy weight range, as determined by body mass index. To obtain more reasonable protein requirements for individuals or groups, recommendations are oftentimes shifted to reflect a standard intake per kg of "normal" or "healthy" body weight. To do this, researchers may choose the closest body weight possible that will place an individual just within a healthy BMI range (18.5-24.9 for adults). As discussed earlier, the lower mortality rates associated with higher BMI values in older adults raise questions about where the healthy BMI range for elderly adults should lie, with some researchers suggesting a range of 22.0 to 27.0 or even higher (WHO 1998).

However, controversy remains among clinical nutrition professionals as to whether adjusting individual body weights will actually provide valid assessments of adequacy and accurate recommendations for intake. As mentioned previously, a higher BMI may be associated with lower all-cause mortality in the elderly, but being overweight or obese, even as an older adult, may place a person at an increased risk for developing major health problems such as insulin insensitivity and a reduced capacity for physical functioning (Janssen 2007; Goya Wannamethee et al. 2004). 
Data collected from NHANES studies is released in two-year increments, with the 2005-2006 data available as the most recent full documentation. The tables below display the range of average protein intakes for adults in the United States, expressed in grams of protein per day and protein as a percentage of total daily energy intake (Table 2.2) as well as grams of protein per kilogram of adjusted, healthy body weight and the proportion of the population with a protein intake below the EAR (Table 2.3). 
Table 2.2 Grams of Protein Consumed per Day (g/day) and as a Percent of Daily Energy Intake (\% en): Usual Intakes from Food Among Adults in the U.S. Data from the NHANES 2005-2006 ${ }^{1,2}$

\begin{tabular}{|c|c|c|c|c|c|c|c|}
\hline & \multirow[b]{2}{*}{ Units } & \multirow[b]{2}{*}{ Mean (SD) } & \multicolumn{4}{|c|}{ Percentiles } & \multirow[b]{2}{*}{95} \\
\hline & & & 5 & 25 & 50 & 75 & \\
\hline \multicolumn{8}{|l|}{ Females } \\
\hline $19-50 y$ & g/day & $74(21)$ & 43 & 59 & 72 & 86 & 111 \\
\hline$(\mathrm{n}=1099)$ & $\%$ en & $15.2(2.8)$ & 11.1 & 13.3 & 15.0 & 16.9 & 20.1 \\
\hline \multirow{2}{*}{$\begin{array}{l}\begin{array}{l}51-70 y \\
(n=579)\end{array}\end{array}$} & g/day & $67(16)$ & 43 & 56 & 66 & 77 & 94 \\
\hline & $\%$ en & $16.0(2.8)$ & 11.8 & 14.1 & 15.8 & 17.7 & 20.9 \\
\hline \multirow{2}{*}{$\begin{array}{l}71 y+ \\
(n=296)\end{array}$} & g/day & $56(13)$ & 37 & 47 & 55 & 63 & 78 \\
\hline & $\%$ en & $15.4(2.4)$ & 11.6 & 13.7 & 15.2 & 16.9 & 19.6 \\
\hline \multicolumn{8}{|l|}{ Males } \\
\hline $19-50 y$ & g/day & $116(30)$ & 72 & 95 & 113 & 133 & 169 \\
\hline$(\mathrm{n}=1127)$ & $\%$ en & $15.2(2.4)$ & 11.7 & 13.6 & 15.0 & 16.6 & 19.4 \\
\hline \multirow{2}{*}{$\begin{array}{l}51-70 y \\
(\mathrm{n}=559)\end{array}$} & g/day & $94(22)$ & 61 & 79 & 92 & 108 & 133 \\
\hline & $\%$ en & $15.3(2.3)$ & 11.8 & 13.7 & 15.1 & 16.7 & 19.3 \\
\hline \multirow{2}{*}{$\begin{array}{l}71 \mathrm{y}+ \\
(\mathrm{n}=334)\end{array}$} & g/day & $78(18)$ & 52 & 65 & 76 & 88 & 110 \\
\hline & $\%$ en & $15.7(1.9)$ & 12.9 & 14.4 & 15.6 & 16.8 & 18.9 \\
\hline
\end{tabular}


Table 2.3 Protein Intake (g/kg healthy body weight per day): Usual Intakes from Food Among Adults in the U.S. Data from the NHANES 2005-2006, Compared with the Estimated Average Requirement (EAR) ${ }^{1,2,3}$

\begin{tabular}{|c|c|c|c|c|c|c|c|c|}
\hline & \multicolumn{8}{|c|}{ Percentiles } \\
\hline & Mean (SD) & 5 & 25 & 50 & 75 & 95 & $\begin{array}{c}\%< \\
\text { Current } \\
\text { EAR }^{4}\end{array}$ & $\begin{array}{c}\%< \\
\text { Proposed } \\
\text { EAR }^{5}\end{array}$ \\
\hline $\begin{array}{l}\text { Females } \\
19 y-50 y \\
(n=1099)\end{array}$ & $1.18(0.35)$ & 0.68 & 0.94 & 1.15 & 1.38 & 1.81 & 4.0 & - \\
\hline $\begin{array}{l}51 y-70 y \\
(n=579)\end{array}$ & $1.06(0.25)$ & 0.68 & 0.88 & 1.04 & 1.22 & 1.50 & 3.8 & - \\
\hline $\begin{array}{l}71 y+ \\
(n=296)\end{array}$ & $0.94(0.22)$ & 0.61 & 0.78 & 0.92 & 1.08 & 1.34 & 9.0 & 63.8 \\
\hline $\begin{array}{l}\text { Males } \\
19 y-50 y \\
(n=1127)\end{array}$ & $1.53(0.41)$ & 0.94 & 1.24 & 1.49 & 1.77 & 2.26 & $<1.0$ & - \\
\hline $\begin{array}{l}51 y-70 y \\
(n=559)\end{array}$ & $1.24(0.28)$ & 0.82 & 1.05 & 1.22 & 1.41 & 1.72 & $<1.0$ & - \\
\hline $\begin{array}{l}71 y+ \\
(n=334)\end{array}$ & $1.07(0.27)$ & 0.69 & 0.88 & 1.04 & 1.23 & 1.56 & 3.8 & 42.5 \\
\hline
\end{tabular}

${ }^{1}$ Data were obtained from adults who had two days of reliable intake form the NHANES 2005-2006

${ }^{2}$ Body weights were adjusted according to USDA appropriate body weight specifications. For individuals whose body mass index (BMI) was below $18.5 \mathrm{~kg} / \mathrm{m} 2$, the weight in kilograms that would place those individuals at the nearest BMI cut point given their height, (either $18.5 \mathrm{~kg} / \mathrm{m} 2$ or $24.9 \mathrm{~kg} / \mathrm{m} 2$ ) was used.

${ }^{3 .}$ Sampling weights were applied and percentile distributions of usual protein intake were generated using C-SIDE.

${ }^{4}$. $\mathrm{EAR}=0.66 \mathrm{~g} / \mathrm{kg}$ body weight

5. Proposed EAR ( $1.0 \mathrm{~g} / \mathrm{kg}$ body weight) consistent with higher RDA proposed by some researchers for older adults (Wolfe 2006).

(Data taken from Berner et al. 2009)

The tables above reflect a few major themes concerning protein intake in the United

States. In Table 2.2 it is easy to see that adults aged 19-50 years, both males and females, consume the largest amount of protein, purely based on total grams of intake, while the 
oldest age groups consume the least. However, when looking at consumption data presented as a percentage of total daily energy intake (Table 2.2) it is interesting to note the relatively similar values present across age groups. Therefore, the decreases in grams of protein intake with age are explained by a reduced daily total energy intake rather than from a selective decrease in protein as a part of the diet.

When comparing this consumption data to DRI recommendations, we can see that very few people consumed less than the current protein EAR, after adjustment to a healthy body weight (Table 2.3). Noteworthy age groups to consider, however, are elderly males and females. The data reveal that 3.8 percent of males 71 and older and 9.0 percent of women 71 and older reported a protein intake that classified them as falling below the EAR of $0.66 \mathrm{~g} / \mathrm{kg} / \mathrm{day}$. While protein malnutrition is not considered prevalent in America, there is evidence that points to a decrease in intake of this macromolecule as people age, if for no other reason than a shift with age to a relatively lower total energy intake (Fulgoni 2008). It is interesting also to consider the shift in the percent of elderly falling below the EAR if it were to be increased to the $1.0 \mathrm{~g} / \mathrm{kg} / \mathrm{day}$ proposed by many researchers (Wolfe 2006; Gaffney-Stomberg et al. 2009). If the protein EAR were increased for older adults, the percentage falling below this recommendation would increase dramatically from less than 10 percent to 63.8 and 42.5 percent of female and male elderly, respectively (Table 2.3).

A second interesting and nutritionally pertinent question to consider when reflecting on national protein intake is not only the quantity of protein Americans are consuming, but the sources of this macronutrient. According to analyses of NHANES data from 2003-2004, 63.52\% of dietary protein consumption in persons ages 19 and over came 
from animal sources, with dairy foods as a leading contributor, followed by beef and poultry. Protein from pork, fish and eggs served as a much less significant contributor to total intake with percentage values of $6.57 \%, 4.60 \%$ and $4.42 \%$ respectively. In this same analysis when men and women 65 years and older were analyzed separately, their protein source distribution followed a very similar pattern to that listed above (Berner et al. 2009).

\subsubsection{Relationship between Dietary Protein and Lean Body Mass in Older Adults}

As mentioned in previous sections, protein plays an extremely vital role in human health and functioning. Of particular interest to many researchers is how exactly dietary protein affects the human body as it ages, both physically and functionally. Sarcopenia is becoming increasingly problematic in our society as more and more Americans are living well into old age, thus warranting investigation into potential mechanisms by which the loss of lean body mass can be slowed or prevented. There are many studies, both clinical and observational, that investigate exactly what role, if any, dietary protein plays in the body composition and preservation of lean body mass in older adults.

\subsubsection{Clinical Research}

In clinical studies of changes in body composition, researchers commonly test elderly men and women at different protein amounts or percentages of total daily calories; it is also common for researchers to compare the effects of high and low protein diets in both young and older subjects. Meaningful outcome measures for such studies include not only measurements of total body and appendicular lean versus fat mass but also blood 
amino acid concentrations, nitrogen balance, protein turnover, and mixed muscle fractional synthesis rates (FSR).

Clinical health trials are expensive and labor-intensive but provide a controlled environment that allows researchers to conclude direct cause and effect. Despite the strengths of this study type, the small sample sizes in nearly all clinical trials warrant skepticism when generalized to an entire population. In a dietary intervention study, ethical problems also arise in prescribing a diet believed to cause adverse health effects. Additionally, the relatively short time period during which clinical interventions are conducted allows for the measurement of muscle anabolism indicators in response to protein ingestion (e.g. FSR, plasma amino acid concentrations) but oftentimes remains too short a period to observe protein's direct effects on preservation of lean mass.

There are many clinical studies supporting the notion that amino acid ingestion improves muscle protein (lean body mass) synthesis in both the young and the elderly. In a small study conducted by Paddon-Jones and colleagues (Paddon-Jones et al. 2004b), researchers attempted to determine if a practical mode of amino acid administration, a single 15-g bolus oral ingestion, could stimulate net muscle protein synthesis to a similar extent in young and elderly subjects. Subjects participating in this study were healthy and independent with no signs or symptoms of sarcopenia and were fasted upon coming in for testing. After background blood samples were obtained on the morning of the test, L-[ring 2H5] phenylalanine was constantly infused for eight hours to observe the rate of its incorporation into proteins. Femoral arteriovenous blood samples were obtained at 10 to 30 minute intervals for three hours before and after the bolus ingestion of $15 \mathrm{~g}$ of essential amino acids (EAA) for the determination of amino acid kinetics and plasma 
concentrations of glucose and insulin. Three muscle biopsies were also taken, two hours prior to EAA ingestion, at the time of ingestion, and three and a half to four hours post, to measure the incorporation of phenylalanine into protein (Paddon-Jones et al. 2004b).

Researchers from this study found that the bolus oral ingestion of $15 \mathrm{~g}$ of EAAs stimulated muscle protein anabolism and produced similar increases in mixed-muscle FSR in both elderly and young individuals. A notable point to consider, however, was the fact that the essential amino acids failed to produce a significant insulin response in the elderly individuals. Also, net phenylalanine balance, a reflection of the relationship between muscle protein synthesis and breakdown, revealed that the elderly subjects responded more slowly to the EAA stimulus but remained in positive net balance for a longer period of time (Paddon-Jones et al. 2004b). Based on these findings, researchers hypothesized that elderly individuals may not be able to increase FSR in proportion to the increase in muscle intracellular phenylalanine concentrations, possibly because of the lack of a measurable insulin response to EAA ingestion. Regardless of these differences, the major practical implication of the study is that elderly people, similar to their younger counterparts, may also derive benefits from the ingestion of amino acids, as specifically measured through protein anabolism or FSR (Paddon-Jones et al. 2004b).

In a similar study, researchers fed young and elderly subjects a 4-oz. serving of 90\% lean ground beef and measured responses in plasma amino acids and muscle protein synthesis (mixed-muscle FSR). Unlike Paddon-Jones, this study concluded no agerelated differences in plasma insulin concentration and that rates of increase and peak concentration of EAAs were actually greater in the elderly than in the young (Symons $e t$ al. 2007). However, the principle outcome of the study fell in congruence with Paddon- 
Jones in that the FSR for both young and old subjects increased similarly (51\%) following the protein-rich meal, pointing to the sustained ability of the elderly body to adequately utilize protein for muscle synthesis (Symons et al. 2007).

Both of these studies, however, fail to adjust for smaller body size and a reduced lean muscle mass in the elderly when compared to younger subjects. Thus, while fractional synthesis levels appear similar in both groups, the older adults actually received a greater bolus of protein per $\mathrm{kg}$ of lean mass. In practical terms, this may mean that if people continue to ingest a similar quantity of high-quality protein as they age, there should be no impairment in protein-synthetic response since they will be eating a greater amount per kg of fat-free mass (Paddon-Jones et al. 2004b; Symons et al. 2007).

In a meta-analysis of several clinical studies, Thalacker-Mercer and Campbell concluded that albumin synthesis rate, a common biomarker of general protein adequacy, is influenced by dietary protein quantity in both younger and older adults, with comparable responses to protein found in both groups. Thus authors concluded that protein appears to have a similar anabolic effect throughout an individual's lifespan (Thalacker-Mercer \& Campbell 2008).

Another interesting situation to consider is the effect of dietary protein on lean body mass during weight-loss diets in older individuals. In a retrospective analysis of data from a randomized, controlled study involving 70 obese, postmenopausal women (ages 50-70), researchers investigated whether dietary protein was associated with a loss of lean mass during a 20-week caloric restriction and exercise weight-loss intervention. After performing a regression analysis of the data to look for an association between total lean mass, appendicular lean mass and protein intake, researchers determined that 
participants who consumed higher amounts of dietary protein lost less lean mass and appendicular lean mass than those consuming less and that a significant positive correlation also existed between protein intake ( $\mathrm{g} / \mathrm{kg}$ body weight $/$ day) and absolute fat mass loss (kg) (Bopp et al. 2008). In unadjusted models, researchers determined that for every $0.1 \mathrm{~g} / \mathrm{kg} /$ day increase in dietary protein, participants lost $0.62 \mathrm{~kg}$ less lean mass. Even after adjusting for exercise-diet intervention group, lean mass and body size, these results remained significant. The overall findings of this analysis suggest an overall negative linear relationship between protein intake and loss of lean mass during periods of negative energy balance (Bopp et al. 2008). These conclusions are also supported in a meta-analysis by Krieger and colleagues, in which it was reported that diets higher in protein (1.05 to $1.20 \mathrm{~g} / \mathrm{kg}$ body weight/day) were associated with greater fat-free mass retention than diets lower in protein $(<0.7 \mathrm{~g} / \mathrm{kg}$ body weight/day) during energy restriction (Krieger et al. 2006).

For those older adults experiencing unintentional weight-loss from decreases in appetite, disease states or reduced physical activity, as in states of physical frailty, it is possible that protein may also help preserve lean tissue. In one experimental study investigating the effects of a carbohydrate-amino acid supplement on a group of older men confined to a sedentary environment (simulating bed rest) for 28 days, researchers found that men receiving the supplement, as opposed to the control group consuming only regular food meals, experienced greater mixed muscle FSR rates, greater retention of lean leg mass and a lesser decline in lower body strength. Thus researchers suggested the possible beneficial effects of essential amino acid and carbohydrate supplementation 
for older adults at risk for developing sarcopenia due to immobility or prolonged bed rest (Paddon-Jones et al. 2004a).

Similar results were found in another uncontrolled study involving supplementation with $22 \mathrm{~g} /$ day of essential amino acids plus arginine. This study included 12 elderly, glucose intolerant men and women as researchers decided that findings of an anabolic effect of amino acids in insulin-resistant subjects would translate into an even greater effect in those with normal insulin sensitivity. Subjects took the supplement containing $11 \mathrm{~g}$ of amino acids twice daily, between meals for a total of 16 weeks with researchers taking and comparing body composition measurements via DXA every fourth week and the following measurements at weeks $0,8,12$ and 16: muscular strength via 1 repetition max testing for leg extension and flexion, muscular endurance via gait speed and biomechanics testing and functional ability via step tests and a timed floor transfer test.

Researchers found significant increases in lean mass, approximately $0.60 \mathrm{~kg}$, on average, between baseline and after 16 weeks of supplementation. Additionally, the subjects experienced significant increases in lower extremity strength (mean $22.2 \pm \mathrm{SE}$ $6.1 \%$ ), usual gait speed (p-value 0.002), timed 5-step test (p-value 0.007) and timed floortransfer test (p-value 0.022). Thus, researchers concluded that supplementation with EAA + arginine improves lean body mass, strength and physical function compared to baseline values in glucose intolerant elderly individuals and that these results can be further generalized to include the elderly population with normal insulin sensitivity. (Borsheim et al. 2008).

In another short-term study looking at whether an increase in protein, but not energy, would improve muscle protein status in frail elderly women, it was determined that the 
frail women's usual "low" protein intake of roughly $0.87 \mathrm{~g} / \mathrm{kg} /$ day was associated with less muscle mass and greater muscle catabolism than that of the experimental group of healthy women who were consuming closer to $1.2 \mathrm{~g} / \mathrm{kg} / \mathrm{day}$. When researchers increased the amount of protein administered to the frail women to levels that matched those of their healthy counterparts, no changes in muscle mass or muscle catabolism were seen, possibly due to the very short (12 day) time span of the intervention. The frail women most likely experienced increased catabolism due to an accommodation mechanism whereby their bodies catabolized muscle to obtain amino acids in an attempt to maintain proper whole-body functioning. One promising finding of the study, however, was the capacity of the frail women to retain nitrogen after increasing protein intakes. This provides evidence to conclude that, while this study was not long enough to witness any changes, if the higher protein diet were sustained over a longer period of time, the women should have the capacity to accrete lean tissue mass (Chevalier et al. 2003).

\subsubsection{Cross-sectional Research}

Cross-sectional studies, while lacking in experimental control and follow-up of individuals over time, provide snapshots of protein consumption and its relationship to lean body mass within a population at a set point in time.

In one observational study of 44 healthy, older white men, researchers examined whether variations in physical activity and dietary protein intake, assessed by means of a uni-axial accelerometer and three-day food record respectively, influenced the rate of decline in total appendicular skeletal muscle mass. Partial correlation analysis revealed that an inverse relationship existed between age and total skeletal muscle mass after controlling for physical activity, dietary protein intake ( $\mathrm{g} / \mathrm{kg}$ body mass) and protein 
expressed as a percentage of total daily energy intake; thus, variations in protein intake and physical activity levels had virtually no effect in modifying the age-related decline in appendicular skeletal muscle mass. While authors acknowledge the need for prospective studies to confirm these results, conclusions from this analysis suggest that protein intake above the RDA is not linked to the preservation of muscle mass in older men (Starling et al. 1999).

Lord and colleagues, in another observational study of 38 healthy, normal weight, sedentary women between the ages of 57 and 75 years, looked at the relationship between protein type (animal or vegetal) and its association with muscle mass index (fat-free mass $\mathrm{kg} /$ height $\mathrm{m}^{2}$ ). DXA techniques were used to measure fat and fat-free mass, creatinine excretion was used to measure muscle protein content and a 3-day diet food log was provided to subjects for a record of dietary intake. Upon conducting both full and partial correlation analyses to measure the relationship between muscle mass index (MMI) and nutritional and body composition variables, researchers found that, while fat and fat-free mass, muscle protein content and total protein intake were all positively correlated with MMI, animal protein intake was the only independent predictor of MMI, explaining 19\% of the variance (Lord et al. 2007). These results suggest that animal protein in particular may be associated with a greater preservation of muscle mass in older adults than nonanimal sources (Lord et al. 2007).

\subsubsection{Longitudinal, Observational Research}

Longitudinal or prospective cohort studies regarding protein intake and lean body mass provide data regarding changes within an individual or group of individuals 
followed over time. This type of study proves very useful, as it accounts for individual physiological changes in response to protein intake.

In the very large Health, Aging and Body Composition study (Health ABC Study), researchers used a DXA scan to assess changes in lean mass (LM) and appendicular lean mass (aLM) over a three-year period in a group of 2732 older adults ages 70-79 years and analyzed these changes with respect to protein intake, assessed via a 108-item, interviewer-administered food frequency questionnaire. The food frequency questionnaire was developed specifically for the Health ABC study from two 24-hour recalls obtained from older, non-Hispanic white and black adults participating in the NHANES III. Trained interviewers in the Health ABC Study used props such as plastic food models to help participants estimate portion sizes for each food and all diets were analyzed for not only total protein intake but for the source of protein intake—animal or vegetal (Houston et al. 2008).

Study findings included associations between protein intake and changes in lean mass over the three-year period with participants in the highest quintile of energy-adjusted protein intake (mean $1.2 \pm \mathrm{SD} 0.4 \mathrm{~g} / \mathrm{kg}$ body mass/day) losing approximately $40 \%$ less LM and aLM than those in the lowest quintile (mean $0.8 \pm \mathrm{SD} 0.3 \mathrm{~g} / \mathrm{kg}$ body mass/day) after controlling for age, sex, race, study site, total energy intake, baseline LM/aLM, height, smoking, alcohol use, physical activity, oral steroid use, prevalent disease and interim hospitalizations (Houston et al. 2008).

Similar to the findings reported by Bopp and Krieger, lower protein intake in a subgroup of weight losers was also associated with greater losses in lean mass than weight losers consuming greater amounts of protein, after controlling for the same 
covariables as listed above. This study is also unique in that it looks at the relationship between protein source and body composition to conclude a significant positive association between only animal protein sources and lean body mass. Results from this study suggest that dietary protein intake, particularly from animal sources, may be a modifiable factor in the prevention of sarcopenia (Houston et al. 2008).

In another longitudinal study following a group of older Chinese men for four years, researchers also found associations between dietary protein intake and changes in body composition with the effects of protein and total energy consumption interacting to determine changes in midarm muscle area, used in this study to predict lean body mass. This means that the effect of protein on change in arm muscle appeared to depend on the level of energy adequacy. The lowest protein intakes and lowest total energy intakes were each independently associated with a greater loss of arm muscle than the highest level of both protein and energy intakes (Stookey et al. 2005).

\subsubsection{Relationship Between Dietary Protein and Muscular Strength, Endurance and Other Functional Measures of Health in Older Adults}

In addition to measures of body composition, muscle preservation and muscle protein anabolism in response to dietary protein intake, functional measures of health, as explained in preceding sections, serve as general quality of life predictors for older adults. There is mixed and very limited direct research surrounding dietary protein and its role, if any, in affecting muscular strength and endurance, mobility and independence in performing basic activities of daily living. There are studies, however, supporting a connection between quantity and quality of lean body mass and increased muscular strength and endurance and functional status. Thus, protein intake may evoke its effects 
on functional measures of health through the roles it plays in the maintenance of lean muscle mass (Goodpaster, et al. 2001; Payette et al. 1998).

\subsubsection{Clinical Research}

While again more difficult to come by than other types of health studies, there is clinical research investigating the physically functional role of dietary protein in older adults, assessed via strength and balance testing, gait speed and gait biomechanics measurements, ability to perform activities of daily living and self-reported functional capacity.

In one nine-week clinical study investigating elderly women and their ability to accommodate to a low protein diet, researchers determined that, compared to women given the RDA amount of protein, those fed half that amount accommodated to the reduced intake through depressed immune functioning, lower neuromuscular and muscular strength functioning and significant losses of lean tissue. The diet for both the low and high protein groups consisted of all whole foods (no supplements) and was completely vegetarian. Subjects in the low protein group received $0.45 \mathrm{~g}$ protein $/ \mathrm{kg}$ body weight while those in the adequate protein group received $0.92 \mathrm{~g}$ protein $/ \mathrm{kg}$ body weight Castaneda et al. 1995).

Muscular strength in this study was assessed via one-repetition max testing for bench press, leg extension and max handgrip dynamometer testing. Lower body power was assessed using a leg extensor power rig bench with which maximum velocity was recorded for each push of the pedal. Researchers found that, unlike the low protein group, women consuming the adequate protein diet did not experience the adverse accommodation effects listed above and actually experienced a $17-21 \%$ increase in max 
handgrip strength as well as significant increases in right (16\%) and left (29\%) leg power outputs (Castaneda et al. 1995).

Another clinical trial conducted by Scognamiglio and colleagues studied the effect of an oral essential and non-essential amino acid mixture on ambulatory capacity assessed via a 6-minute timed walk test, myocardial function based on left ventricular ejection fraction, perceived mobile capacity via responses on a walking impairment questionnaire and isometric strength estimated with use of a handgrip dynamometer. In this study 44 sedentary, elderly men and women 65 years and older subsequently reporting a lower health-related quality of life were divided into two groups with each person receiving either an amino acid supplement (mixture of essential and non-essential AAs) amounting to 12 grams of amino acids per day or a placebo pill. Subjects continued treatment for a total of 3 months with physical capacity assessed at baseline and again at the completion of the trial (Scognamiglio et al. 2004).

Results from this trial included a significant increase in all measures of functional health at the end of 3 months in those subjects supplementing with amino acids. Subjects who had consumed the amino acid supplement could walk further, reported greater ambulatory ease on the walking impairment questionnaire, and experienced significant increases in grip strength. In addition, the left ventricular response to exercise normalized during amino acid ingestion in $75 \%$ of subjects who experienced problems with this at baseline. Thus, protein ingestion proved to be a non-pharmacological intervention in maintaining fitness in the elderly (Scognamiglio et al. 2004).

Campbell and colleagues investigated another avenue of this issue as they studied the effects of lower and whole body strength training on muscle hypertrophy and protein 
metabolism, while subjects were fed the RDA amount of protein. In this 14-week controlled diet and exercise study of 12 men and 17 women ages 55 to 78 , subjects were randomly assigned to one of three groups: sedentary, lower body resistance training (LBRT) or whole body resistance training (WBRT) with the resistance training groups performing strength sessions three times per week. The LBRT group performed unilateral knee extension and bilateral seated leg curl exercises only while the WBRT group performed unilateral knee extension and bilateral seated leg curl exercises (exactly the same as the LBRT group), as well as seated chest press and seated arm pull exercises. For each exercise, two sets of eight repetitions at $80 \%$ of each individual's one repetition maximum were performed, followed by a third set of repetitions to voluntary muscular fatigue. If twelve repetitions were successfully completed for set three, the training resistance was increased by $5 \%$ for the next exercise session (Campbell et al. 2002).

Throughout the duration of the trial, diet was also controlled to maintain protein intake at $0.8 \mathrm{~g}$ protein $/ \mathrm{kg} / \mathrm{day}$. Each subject was provided all food and beverages, using a rotating schedule of three menus, portioned to provide sufficient total dietary energy to maintain body weight, $0.8 \mathrm{~g}$ protein $/ \mathrm{kg} /$ day and a non-protein energy content of $60 \%$ carbohydrate and $40 \%$ fat (Campbell et al. 2002).

Researchers measured muscle hypertrophy via mid-thigh and mid-arm muscle area (from CT scan) and strength gains via one-repetition max testing at baseline and again during weeks 6 and 12 and found that subjects consuming the protein RDA while engaging in resistance training increased muscle strength and hypertrophy while those consuming the RDA but remaining sedentary did not. No significant strength or hypertrophy differences were found between the LBRT and WBRT groups thus 
indicating that the number of muscle groups trained does not seem to influence the hypertrophy response. These findings point to two relevant conclusions. One, that it is possible for elderly people to increase strength and hypertrophy through exercise and two, that the current protein RDA of $0.8 \mathrm{~g} / \mathrm{kg} / \mathrm{day}$ may not alone be adequate in maintaining lean muscle mass and preserving muscular strength in older adults (Campbell et al. 2002).

Similar to Campbell's study, Verdijk and colleagues performed a clinical trial to assess whether the administration of $10 \mathrm{~g}$ of protein, as a liquid supplement of casein hydrosylate, administered just before and immediately following a strength training session in 26 older adult men (72 \pm 2 years), would increase skeletal muscle hypertrophy and strength. Subjects participated in three strength-training sessions per week for a total of 12 weeks while receiving either a placebo beverage or a beverage containing $10 \mathrm{~g}$ of protein in the form of casein hydrosylate both five minutes before and five minutes after exercise (total 20g protein per exercise session). Upon conclusion of the 12-week program, one-repetition maximum strength improved by $25-35 \%$ in both groups and no statistically significant difference in strength existed between them. Thus, timed protein supplementation before and after exercise did not appear to have an effect in augmenting skeletal muscle mass or strength (Verdijk et al. 2009).

\subsubsection{Cross-sectional Research}

Cross-sectional data surrounding dietary protein intake and functional measures of health in older adults allow researchers to observe the physical responses to real-life food intake outside the confines of a clinical trial. Thus, while they provide data from trials 
that are less controlled, researchers are still able to observe day-to-day food habits and what direct effects, if any, they have on health.

Declines in functional measures, as mentioned in previous sections, are often used as indicators of frailty. In one large cross-sectional study investigating low nutrient intake as a component of frailty in a population of 802 elderly persons, researchers concluded that low intakes of energy and specific nutrients, specifically vitamins D, E, C, folate and protein, were independently associated with frailty even after adjustment for energy intake (Bartali et al. 2006). In this study, researchers defined low nutrient intake as the amount corresponding to the lowest sex-specific quintile of intake of those participating in the trial; for protein, these values were $<66 \mathrm{~g} /$ day for men and $<55 \mathrm{~g} /$ day for women. Nutrient intake was assessed using an interviewer-administered food frequency questionnaire administered in the participants' home.

In the assessment of frailty, researchers used both questionnaires and measured functional testing. Frail participants were classified based on the operational definition developed by Fried and colleagues as an individual experiencing two or more of the following criteria: exhaustion (self-reported feeling that "everything I did was an effort," at least three times per week in the last month), low physical activity (sedentary or performing light activity less than one time per week), poor muscle strength (assessed via grip strength testing), and low walking speed (assessed as time to walk 15 feet) (Bartali et al. 2006).

Upon statistical analysis of the data, researchers found that the specific odds ratio for low protein intake and its relationship to frailty was high at 1.98 (p-value 0.009), 
indicating that older people consuming low amounts of protein nearly doubled their risk for developing physical frailty (Bartali et al. 2006).

While a limited amount of research exists directly linking protein intake to physical functioning in older adults, there have been studies concluding possible improvements in physical functioning with increased protein intake. In the literature, many papers have shown a positive association between lean body mass and muscular strength; consequently, studies linking protein intake to improvements in body composition and preservation of lean mass may also serve as a basis for assuming protein's role in improving functional measures of health.

\subsection{Justification and Practical Significance of Research}

Elderly adults experience physiological shifts with age that include changes in body composition and anthropometric measures as well as functional ability and muscular strength and endurance. Several research groups have demonstrated relationships between adequate consumption of dietary protein and the preservation of lean muscle mass, a component of health that may be in and of itself associated with the maintenance of strength and functional capacity in the elderly. Other published research, although limited, connects protein intake to the maintenance or even increase in strength in the elderly, both with and without the accompaniment of resistance training.

Is it possible then that the maintenance of lean body mass and healthy functioning in an older population can be ameliorated by adequate dietary protein intake and, if so, just how much and what types of protein should the elderly be consuming?

There is currently a lack of published data, particularly nationally representative published data, regarding the relationship between protein intake and lean body mass and 
the relationship of both protein intake and lean body mass to physical functioning in older adults. The increased numbers of Americans living well into old age and the prevalence of sarcopenia and its related conditions in this country justify these as relevant and compelling issues that warrant further investigation.

\subsection{Main Research Questions and Hypotheses}

The aim of this research was to shed light on the questions surrounding protein, lean body mass and physical functioning in American adults, with a particular interest in older Americans $60+$ years, those most at risk for low muscle mass and poor functional mobility. Three main research questions were chosen based on the need for information regarding protein, lean body mass and physical functioning in a nationally representative sample of adults and the availability of publicly-released NHANES data. The three main research questions and their respective hypotheses are reported below:

1. What is the relationship between the amount and type of dietary protein intake $(\mathrm{g} / \mathrm{d}$, $\%$ of total daily energy, $\%$ contributed by animal foods) and the amount of lean body mass (Total/appendicular lean mass, Total/appendicular Muscle Mass Index, and percent lean body mass) in U.S. adults?

Hypothesis: Greater dietary protein consumption, controlling for total energy intake, age, gender, body size, race/ethnicity, education and physical activity, will be associated with greater lean body mass. 
2. What is the relationship between amount of lean body mass (Total/appendicular lean mass, Total/appendicular Muscle Mass Index, and percent lean body mass) as estimated using DXA and self-reported physical functioning, as assessed via NHANES selfadministered questionnaires in older U.S. adults?

Hypothesis: Greater lean body mass, controlling for body size, age, race/ethnicity, education, general health status and physical illness/injury status, will be associated with better self-reported physical functioning.

3. If data show a positive relationship between lean body mass and self-reported physical functioning, what is the relationship between the amount of dietary protein consumed (grams/day, $\%$ of total daily energy, $\%$ contributed by animal foods) and self-reported physical functioning, assessed via NHANES self-administered questionnaires?

Hypothesis: Greater dietary protein consumption, controlling for body size, total energy intake, age, race/ethnicity, education, general health status and physical illness/injury status, will be associated with better self-reported physical functioning. 


\section{Materials and Methods}

Data used in the analysis of protein intake, lean body mass and physical functioning were taken from participants in the National Health and Nutrition Examination Survey (NHANES), 2003-2004. NHANES technicians surveyed approximately 5,000 Americans from 15 counties during each exam year, with subjects representative of the U.S. population of all ages. In the 2003-2004 examination, special emphasis was placed on adolescent health and the health of older Americans. To achieve this and produce reliable statistics for these groups, adolescents aged 15-19, older persons $60+$ years and African Americans and Hispanics were over-sampled for the survey (CDC 2007a).

Exam components consisted of an at-home health interview during which the examiner collected demographic, socioeconomic, dietary and health-related information. This was followed by a more thorough exam conducted at a mobile examination center (MEC) that included medical and dental exams, physiological measurements, dietary assessment and extensive lab tests. Information from the survey was assembled and made publicly available online through the CDC.

The NHANES data from 2003-2004 were chosen for use in this research due to this survey cycle's inclusion of exam components relevant to our research objectives. While dietary data and self-reported physical functioning were collected similarly in each survey cycle since 1999, lean body mass measurements obtained via DXA scan differed between years. In 2003-2004, researchers used a DXA scan to obtain estimates of lean body mass, divided extensively by body region while in other years scans estimated only bone mineral density or were not used at all. The following sections describe the 
NHANES protocol for conducting exams and managing data as well as descriptions of and rationale for the statistical models used in analyzing that data for this research.

\subsection{NHANES Collection and Management of Data}

The NHANES measures used in this research are described in detail in the following sections. These descriptions include exam protocols, NHANES inclusion criteria and NHANES methods for processing collected data.

\subsubsection{The Dietary Interview}

The objective of NHANES researchers during the dietary interview was to collect detailed diet information from all NHANES participants. New in 2003-2004 was the collection of two days of diet information to obtain estimates of usual intake of nutrients. The first interview, conducted in the MEC, asked participants to recall the type and amount of all food and beverages consumed in the previous 24 hours. The second interview asked for the same information but was conducted as a phone interview three to ten days following the initial screening but not on the same day of the week as Interview

One. All NHANES participants were included in this assessment, with caretakers answering for children less than 6 years of age and assisted interviews for children six to eleven years (CDC 2007b).

Interview data files were processed through USDA's Food and Nutrient Database for Dietary Studies (FNDDS 2.0), used to code individual foods and portion sizes to calculate intakes of specific nutrients. The underlying nutrient values for FNDDS 2.0 were based on values in the USDA National Nutrient Database for Standard Reference, release 18. Of the 12,761 people selected for participation in the 2003-2004 NHANES, 
9,643 responded to the MEC examination and data collection but only 8,354 provided complete dietary intake data for days 1 and 2 (CDC 2007b).

\subsubsection{The Dual Energy X-ray Absorptiometry (DXA) Scan and Multiple Imputation of Data}

NHANES DXA scans were administered using a Hologic QDR-4500A fan-beam densitometer with Hologic version 9.26:a3 software. Participants were asked to lie on the examination table in a supine position while multiple low-radiation $\mathrm{x}$-ray passes were made over the body. The ratio of tissue penetration of the x-ray scans was used to distinguish bone from soft tissue and the percent fat in the soft tissue once bone was excluded. Body composition for specific body regions was obtained and values for these as well as total lean mass, excluding bone mass, total fat mass and total percent body fat were reported in the final dataset.

NHANES technicians administered DXA scans to all eligible survey participants 8 years and older. Exclusion criteria for the exam included:

\section{Pregnancy}

2. Self-reported history of radiographic contrast material (barium) use in past seven days

3. Self-reported nuclear medicine studies in the past three days

4. Self-reported weight over 300 pounds or height over 6'5" (DXA table limitations) 
In subjects who were eligible to perform the scan, several additional factors were potentially responsible for invalid body composition estimations for one or more body regions. These reasons included:

1. Jewelry and/or other objects were not removed

2. Non-removable objects (including prostheses, implants or casts) were present

3. Excessive x-ray noise due to obesity (i.e. DXA beam could not penetrate layers of abdominal fat)

4. Arm/leg overlap

5. Body parts out of scan region

6. Positioning problem (head, arms, hands or feet turned)

7. Other (including participant motion, unknown artifacts and deformities)

(CDC 2008a)

These invalidity factors were accounted for and for these body regions, data was imputed through a complicated process described below.

\subsubsection{Justification for Multiple Imputation of DXA Data}

Due to an unusually high amount of non-random missing and invalid data, NHANES researchers could not exclude subjects with missing data due to the potential for bias. The percentages of missing data increase with increasing age and BMI, as shown in Table 3.1 and Table 3.2 below. The tables indicate NHANES participants with one or more missing body regions. 
Table 3.1 Percentages of NHANES

participants from 1999 to 2004 with

data missing, by age group

\begin{tabular}{|l|c|c|c|}
\hline \multirow{2}{*}{ Years } & \multicolumn{3}{|c|}{ Percentage } \\
\cline { 2 - 4 } & $1999-2000$ & $2001-2002$ & $2003-2004$ \\
\hline $8-11$ & 10 & 7 & 7 \\
\hline $12-15$ & 11 & 10 & 10 \\
\hline $16-19$ & 15 & 14 & 14 \\
\hline $20-29$ & 18 & 18 & 20 \\
\hline $30-39$ & 21 & 21 & 21 \\
\hline $40-49$ & 22 & 21 & 22 \\
\hline $50-59$ & 25 & 23 & 27 \\
\hline $60-69$ & 28 & 28 & 25 \\
\hline $70-79$ & 30 & 27 & 36 \\
\hline $80+$ & 41 & 41 & 45 \\
\hline
\end{tabular}

Taken from Technical Documentation for the 1999-2004 Dual Energy X-Ray Absorptiometry (DXA) Multiple Imputation Data files (CDC 2008b)

Table 3.2 Percentages of NHANES participants from 1999 to 2004 with data missing, by body mass index (BMI) category

\begin{tabular}{|c|c|c|c|}
\hline & \multicolumn{3}{|c|}{ Percentage } \\
\hline BMI Category & $1999-2000$ & $2001-2002$ & $2003-04$ \\
\hline$<18$ & 22 & 11 & 26 \\
\hline $18-24.9$ & 18 & 13 & 22 \\
\hline $25-29.9$ & 18 & 15 & 20 \\
\hline $30-34.9$ & 23 & 19 & 21 \\
\hline $35.0-39.9$ & 42 & 42 & 38 \\
\hline$\geq 40$ & 81 & 78 & 77 \\
\hline
\end{tabular}

Taken from Technical Documentation for the 1999-2004 Dual Energy X-Ray Absorptiometry (DXA) Multiple Imputation Data files (CDC 2008b)

Rather than deleting those with missing or invalid data, NHANES technicians used multiple imputation, with five plausible but non-measured sets of replacement data, for missing or invalid body regions. The process of multiple imputation, described in detail below, has many strengths that justify its use for handling missing data. Imputation adjusts for differences between respondents and nonrespondents and allows researchers 
to investigate possible reasons for missing data, whereas listwise deletion fails to do so. Multiple (versus single) imputation allows uncertainty due to imputation to be reflected in the analysis as each of the multiple imputations for a body region varies from the next. Creating a multiply-imputed dataset also ensures that nonresponse will be handled consistently across data users rather than by choice deletions that may vary between researchers. Lastly, imputation has been shown to be more effective than re-weighting as it uses statistical relationships between measured survey variables to produce more accurate predictions of missing values (CDC 2008b).

\subsubsection{Procedure for Multiply Imputing NHANES DXA Data}

The multiple imputations for NHANES DXA data were created using sequential regression multivariate imputation (SRMI), broken into ten age-gender groups for the procedure: males and females ages 8-11, 12-19, 20-29, 30-39, 40-59 and 60+. After imputation, the data for the age-gender groups were concatenated. In this SRMI process, fully observed variables are used together in a fitted regression model with newly imputed variables to generate additional, imputed data.

Within each age and gender group, variables with missing values were picked out and ordered by the amount of missing data, from least to most. For example if, within females $60+$ years, 100 subjects were missing data for right arm lean mass (RALE), 125 were missing data for right leg lean mass (RLLE), and 150 were missing data for left leg lean mass (LLLE), the missing variables would be ordered as follows:

1. RALE (Subjects with missing data $=100$ )

2. $\operatorname{RLLE}($ Subjects with missing data $=125)$

3. LLLE (Subjects with missing data $=150$ ) 
Next, the imputation process for these variables would start as a series of iterations, beginning with the variable with the fewest number of missing data values (RALE). In the first iteration, the regression of RALE on the fully observed variables (chosen as those in the dataset relevant to the missing values) would be fitted to the cases with RALE observed and the missing values for RALE are imputed. Next, the regression of RLLE on the fully observed variables and RALE (including imputed values for RALE) is fitted to the cases with RLLE observed and the missing values for RLLE are imputed. Lastly, the process would include a regression of the variable with the greatest amount of missing data (LLLE) on the fully observed variables, RALE and RLLE then fitted to the cases with LLLE observed to generate imputed values for missing LLLE data. In each regression, all variables, imputed and observed, except for the variable to be imputed during that iteration, are used as predictors in the regression model. By including every variable except for the one being imputed during the current iteration, SRMI builds in relationships among variables. This is particularly important with the DXA data since so many of the variables (i.e. body weight, total lean mass, lean mass of arms/legs) are so highly interrelated (CDC 2008b).

The process of multiple imputation is strengthened by the inclusion of a large number of observed predictor variables. NHANES researchers chose to use a wide range of variables in their imputation including demographic, socioeconomic and geographic variables as well as body measurements, indicators of health, food/beverage and medication intake, blood test results and variables related to the design of the NHANES sample (See Appendices A and B for complete list). 
To evaluate the validity of the imputed values, NHANES researchers plotted DXA data (trunk fat, total fat) versus other body measures (weight, BMI, circumferences) in subjects with both observed and missing data. All plots created showed that the relationship involving the imputed DXA values were similar to those of the non-missing data. NHANES researchers looked at the reasonability of data values (i.e. sum of imputed DXA regions should be highly correlated with measured body weight). This was the case for all ten age-gender groups, with a graphical example of men 20-39 shown in Figure 3.1 below.

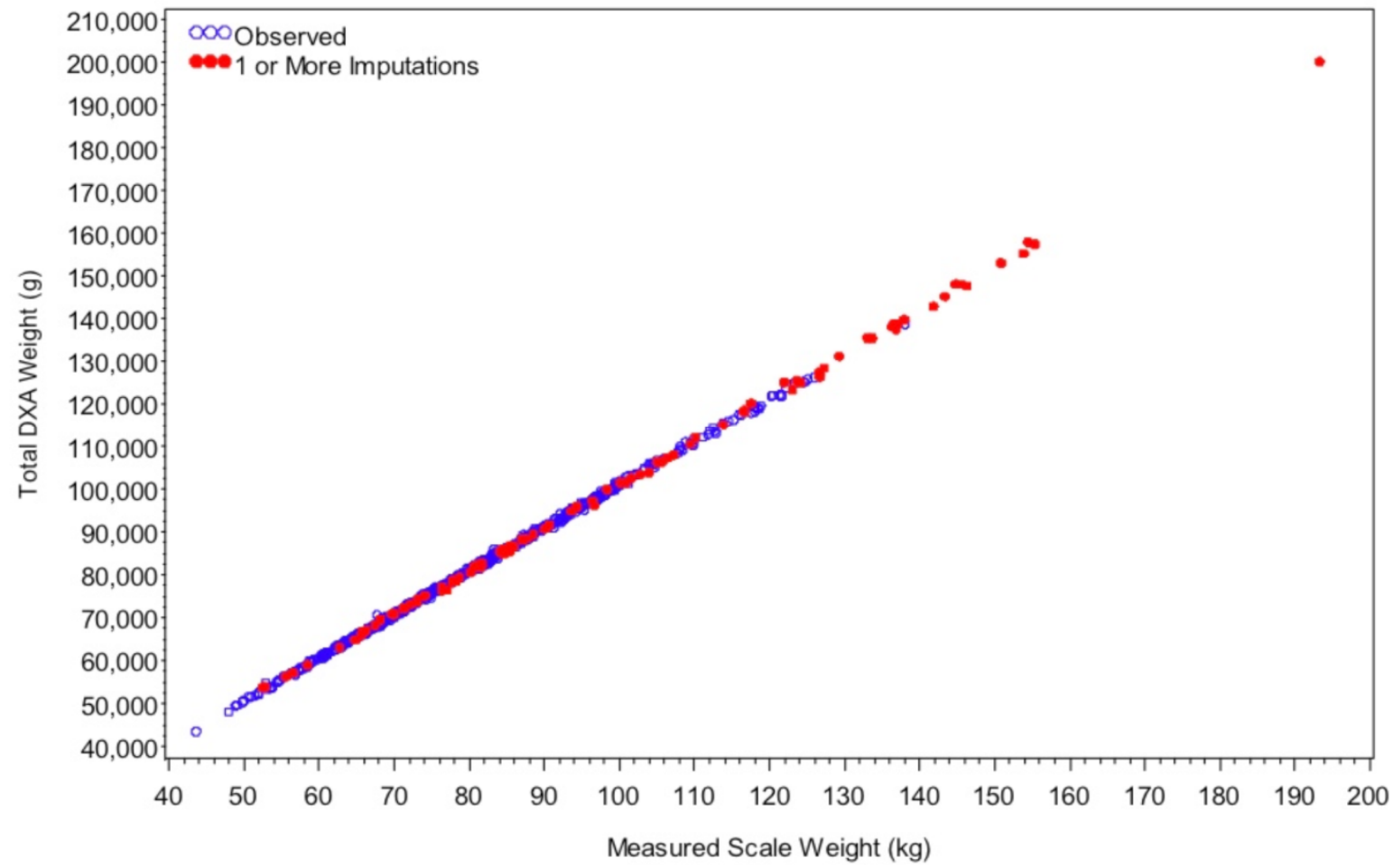

Figure 3.1 Total DXA Weight (g) versus Measured Scale Weight (kg) First Imputation for Males 20-39

Taken from Technical Documentation for the 1999-2004 Dual Energy X-Ray Absorptiometry (DXA) Multiple Imputation Data files (CDC 2008b) 
In addition to plots of DXA variables versus other measured data in subjects with and without missing data, numerical summaries (mean, min, max, percentiles) for the imputed and non-imputed DXA data were generated and compared. These comparisons revealed a definite similarity between imputed and non-imputed values, thereby strengthening the validity of data generated through multiple imputation (CDC 2008).

The final NHANES 2003-2004 DXA data contains five complete data files for each subject. If subjects were missing data for a particular body region variable, each of the five data files for that variable contains a different imputed value. If subjects were not missing data, all five data files contain identical, true measured values. In the analysis of the data, each estimation procedure must be carried out five times, one time for each completed dataset. Aside from this major difference, statistical analysis of a multiply imputed dataset is similar to analyzing a dataset with no missing values (CDC 2008b).

\subsubsection{The NHANES Physical Functioning Questionnaire}

Self-reported physical functioning was chosen for this research as the most appropriate measure of muscular strength, endurance and function in older adults, due in part to the NHANES 2003-2004 removal of more direct measures of strength from its exam component. The Physical Functioning Questionnaire was designed as a way of assessing an individual's level of disability, as reported as difficulty performing specific, everyday tasks (CDC 2008c). The questionnaire was administered during the household interview to NHANES participants of all ages, with answers to initial questions determining the course of the interview in a specific skip pattern (See Appendix $\mathrm{C}$ for complete questionnaire). 
The questions chosen as most relevant for our research involved the ranking of difficulty in performing specific tasks of everyday living (PFQ.061). These specific questions were asked of participants 60 years of age and older or participants less than 60 years of age who also reported "yes" to one or more of the following four questions:

PFQ.049 Does a physical, mental or emotional problem now keep you from working at a job or business?

PFQ.054 Because of a health problem, do you have difficulty walking without using any special equipment?

PFQ.057 Are you limited in any way because of difficulty remembering or because you experience periods of confusion?

PFQ.059 Are you limited in any way in any activity because of a physical, mental or emotional problem?

Additionally, if subjects answered "yes" to question PFQ.054 asking about difficulty walking without the use of special equipment, they were not asked to rate their difficulty performing two of the functioning tasks: walking $1 / 4$ mile and walking up ten steps without resting. Thus, people reporting difficulty walking without special equipment have missing data for these two questions in the final dataset (CDC 2003).

Physical Functioning Questionnaire items used in our analyses were chosen to reflect self-reported mobility, strength and dynamic balance and are consistent with items commonly used as reliable measures of functional ability in older adults (Hoeymans et al. 1996; Sayers et al. 2004; Alexander et al. 2000). 
The four physical limitation questions chosen for use in our analyses included difficulty ratings of the following tasks:

PFQ061.B Walking for a quarter of a mile (that is about two or three blocks)

PFQ061.C. Walking up ten steps without resting

PFQ061.E Lifting or carrying something as heavy as ten pounds (like a sack of potatoes or rice)

PFQ061.I Standing up from an armless straight chair (CDC 2003).

Participants questioned about difficulty in performing these tasks of everyday living were asked to rate their difficulty performing each by themselves and without the use of any special equipment. They were told not to take into account temporary conditions such as broken limbs or pregnancy. Response options for each task included: "no difficulty," "some difficulty," "much difficulty," "unable to do," "do not do this activity," "refused" and “don't know" (CDC 2003).

\subsection{Research Models}

All statistical analyses were run using SAS 9.2 and SAS-callable SUDAAN 10.1 software to accommodate the NHANES complex weighted survey design.

\subsubsection{Lean Body Mass as Predicted by Protein Intake}

SUDAAN's PROC REGRESS was used for all analyses of protein intake and its relationship to lean body mass. SUDAAN version 10.1 contains a built-in option for analyzing multiply imputed data. A Taylor Series variance estimation method for multiply imputed data, using a "with replacement" design, was used. As described in 
Section 3.1.2.2 above, multiply imputed data was separated into five datasets, one for each of the five DXA values, imputed or non-imputed and identical, assigned to each subject. Each of the five complete datasets was analyzed separately and then combined using SUDAAN to produce a single statistical summary of values that incorporate the uncertainty caused by missing data. Using PROC REGRESS, SUDAAN calculated regression coefficients from each of the five datasets, as if they were standard datasets with no missing values. These coefficients were then combined using SUDAAN into a single summary statistic by taking the mean of the five individual regression coefficients. The combined standard error for the summary regression coefficient was calculated based on the within- and between-imputation variances of the five imputed values (CDC 2008b).

NHANES two-year sample weights were used to account for the nationally representative nature of the NHANES survey.

The data were also sorted by age and gender group, divided based on standard age groupings used for protein DRI ranges, and separated into the following groups of datasets for analysis:

Age group 1 Males: 19-50 years $(n=648)$

Age group 1 Females: $19-50$ years $(n=578)$

Age Group 2 Males: 51-70 years $(n=435)$

Age Group 2 Females: 51-70 years $(n=474)$

Age Group 3 Males: $71+$ years $(n=280)$

Age Group 3 Females: $71+$ years $(n=294)$ 
Participants less than 19 years of age were not used in any of the analyses. Descriptions of the variables used in the research models are presented in the following sections.

\subsubsection{Response Variables}

The NHANES 2003-2004 DXA estimate of total lean body mass in grams, excluding bone (DXDTOLE), was chosen as a primary response variable. In addition, select DXA and body measurement data were used to create four new response variables, all unique expressions of lean body mass. These four new variables included: appendicular lean mass in grams (DXDALM), percent lean mass (DXDTOPL), muscle mass index (DXDMMI) and appendicular muscle mass index (DXDAMMI). These measures of lean body mass were chosen based on their hypothesized relationship to protein, strength and physical functioning and their widespread use in other published research (Bopp et al. 2008; Doherty 2003; Houston et al. 2008; Jannsen et al. 2002; Krieger et al. 2006; Lord et al. 2007; Payette et al. 1998; Starling et al. 1999; Zoico et al. 2004). The appendicular measures in particular were thought to be positively associated with mobility and basic performance of activities of daily living (ADLs), as they provide measures of muscle mass in only the arms, legs, hands and feet. Descriptions of the five response variables used in regression analyses of protein and its relationship to lean body mass are described below:

1. Total lean body mass $(\mathrm{g})$ : Total body fat-free muscle mass, excluding bone

2. Appendicular lean body mass (g): Fat-free muscle mass of the arms, legs, hands and feet, excluding bone

DXDALM $=[$ left arm and leg lean mass $(\mathrm{g})+$ right arm and leg lean mass $(\mathrm{g})]$

* Arm lean mass includes the hands and leg lean mass includes the feet. 
3. Percent lean body mass (\%): Percent of total body mass that is fat-free, non-bone mass

DXDTOPL $=[$ Total body lean mass $(\mathrm{kg}) /$ Weight $(\mathrm{kg})] \times 100$

4. Muscle Mass Index $\left(\mathrm{g} / \mathrm{m}^{2}\right)$ : A ratio of total body fat-free muscle mass ( $\mathrm{g}$ ), excluding bone, to height $\left(\mathrm{m}^{2}\right)$

DXDMMI $=\left[\right.$ Total body lean mass $(\mathrm{g}) /\left(\right.$ Height $\left.(\mathrm{m})^{2}\right]$

5. Appendicular Muscle Mass Index $\left(\mathrm{g} / \mathrm{m}^{2}\right)$ : A ratio of the fat-free muscle mass $(\mathrm{g})$ of the arms, legs, hands and feet, excluding bone, to height $\left(\mathrm{m}^{2}\right)$

DXDAMMI $=\left[\right.$ Lean mass of the arms, legs, hands and feet $(\mathrm{g}) /\left(\right.$ Height $\left.(\mathrm{m})^{2}\right]$

\subsubsection{Main Predictor Variables}

NHANES 2003-2004 protein intake data, described above as data from two 24-hour recalls, were used to create primary predictor variables for all analyses. From this data, three protein intake variables were created and used, all two-day averages: total grams of protein intake per day (gPROAVE), protein as a percent of total daily energy (P_TKCAL_2dAVE) and protein as a percent contributed by animal foods (AVGPCTANIMAL). Descriptions of the three predictor variables used in regression analyses of protein and its relationship to lean body mass are described below:

1. Total protein intake (g): Total grams of protein consumed per day, as an average from two 24-hour recalls.

gPROAVE $=[($ Protein intake Day $1(\mathrm{~g}))+($ Protein intake Day $2(\mathrm{~g})) / 2]$

2. Protein as a percent of total daily energy (\%): Percent of total daily calories obtained from protein, as an average from two 24-hour recalls. 
P_TKCAL_2dAVE $=[($ kcals from protein Day $1 /$ total kcals Day 1$)+($ kcals from protein Day 2 / total kcals Day 2)] x 100

3. Protein as a percent contributed by animal foods (\%): Percent of an individual's protein intake that came from all animal sources (meat, fish, poultry, eggs and dairy), as an average from two 24-hour recalls. This variable, AVGPCTANIMAL, was taken from Berner et al. 2009.

\subsubsection{Covariables}

Covariables from NHANES demographic, body measurement and physical activity monitor datasets were used in the regression analyses due to their hypothesized relationship to both lean body mass and protein intake.

Total energy intake was used as a quantitative variable, defined as an average daily calorie intake from two days of 24-hour recall data. This was used as a covariable in all analyses of protein intake and lean body mass to control for the relationship of total energy consumption to both the main predictor and the response variable. In this way, models were able to test the relationship of protein intake to lean body mass, controlling for the amount a person was eating.

Body Mass Index or weight was used to control for body size and its relationship to lean mass and protein intake in analyses of protein intake on total, appendicular and percent lean mass. Choosing a covariable to control for size was challenging in that typical controls for size included body weight, also a component of the lean mass response variables. It was decided though that BMI, which also took into account height, was useful in accounting for total body mass, which included not only lean tissue but also 
fat tissue and bone mass. In this way, analyses were able to show the specific relationship of protein to lean mass, controlling for fat and bone. In this way, an increase in lean body mass as predicted by protein intake would not merely reflect a larger person. When total and appendicular lean mass served as main response variables, BMI was used as a continuous covariable to control for body size. BMI can be used as a continuous variable in this case because it tends to increase and decrease with an increase or decrease in lean mass.

In analyses where percent lean mass was used as a response variable, BMI was grouped based on standard healthy ranges (WHO 1998) into the following categories: $<18.5$ (Underweight)

18.5-24.9 (Normal weight, referent group) 25.0-29.9 (Overweight) $>30.0$ (Obese)

BMI was used categorically in this case because it does not necessarily increase or decrease together with percent lean mass. A person could potentially have a very high BMI with either a high or low percent lean mass. Used categorically, BMI still controls for size so that the relationship between protein and lean body mass can be analyzed within a given BMI group. When BMI was used as a categorical variable in protein and lean body mass analyses, the normal weight range (18.5-24.9) was set as the referent group.

In analyses of protein intake and its relationship to total and appendicular muscle mass index, weight rather than BMI was used as a continuous covariable to control for size. This was done to eliminate the redundancy in using height as part of both muscle 
mass index $\left(\mathrm{g} / \mathrm{m}^{2}\right)$ and BMI $\left(\mathrm{kg} / \mathrm{m}^{2}\right)$, had we chosen BMI to control for size as was done in other analyses mentioned above. Weight was used quantitatively due to its parallel increase or decrease with muscle mass index. As weight increases, muscle mass per unit height generally increases as well.

\section{Beginning with NHANES 2003-2004, physical activity level and intensity was} assessed via physical activity monitors (PAMs), a form of pedometer worn at home for seven consecutive days. The PAM measured intensity and duration of common locomotor activities such as walking and jogging. However, as an accelerometer it could only detect actual motion, therefore time spent engaging in activity on stationary equipment, strength training or any other static activity (i.e. yoga, pilates) was not recorded. In addition to this, the monitors could not get wet and therefore were not useful in recording activity done in the water (CDC 2006). Despite its limitations, the PAM average intensity value, the average intensity of all activity/movement performed during the time the monitor was worn, was the best available covariable to control for the relationship between physical activity and lean muscle mass. The PAM average intensity value ranges from 0 to 32,767 and corresponds to an intensity value based on metabolic equivalents (METS). It was used as a quantitative variable in all analyses of protein and lean body mass.

Age, race/ethnicity and education level were used as covariables in all analyses to control for physiological, genetic and socioeconomic differences among NHANES participants. Since participants were already divided into datasets based on age group, age was used as a quantitative variable within each. Race/ethnicity and education level 
were both used categorically, with non-Hispanic White and the lowest level of education set as reference levels.

\subsubsection{Summary of Analyses Run: Protein Intake as Predictive of Lean Body Mass}

As mentioned above, analyses were run by age and gender groups for all combinations of predictor and response variables to examine the relationship between protein and lean body mass, expressed in a variety of ways. This allows for the examination of not only whether the quantity of protein intake but the amount of protein as a proportion of total calories and the type of protein show any relationship to lean body mass. Several measures of lean body mass were used as they were all hypothesized to positively relate to physical functioning. They examined not only total mass but total mass per unit height and appendicular muscle mass alone.

The following summarizes the analyses performed, all displayed in tabular format in the results section:

Age-gender groups:

Males and Females

- $19-50$ years

- 51-70 years

- $71+$ years

Response Variables:

1. Total body lean mass

2. Appendicular lean mass

3. Muscle Mass Index 
4. Appendicular Muscle Mass Index

5. Percent total body lean mass

Main Predictor Variables:

1. Total grams of protein intake

2. Protein as a percent of total energy

3. Protein as a percent contributed by animal foods

Total number of analyses $=90$

(6 age-gender groups $\mathrm{x} 3$ predictor variables $\mathrm{x} 5$ response variables)

\subsubsection{Self-reported Physical Functioning as Predicted by Lean Body Mass in Older Adults}

Lean body mass is presumed to be associated with general health and well-being as it functions as a primary mover of everything from the arms and legs to the respiratory, digestive and circulatory systems and also plays a role as a major heat producer for the body, vital for the proper functioning of enzymes and metabolism as a whole (Saladin 2004). When examining lean body mass, especially in older adults, the greatest area of interest is in its relationship to physical functioning, particularly its ability to predict ease in performing basic ADLs and moving about independently.

Beginning in 2003-2004, NHANES replaced direct tests of muscular strength and endurance with the physical activity monitor (PAM) described previously, as well as a series of physical health, functioning and activity questionnaires. It was decided for this research that the PAM was not directly reflective of functional ability, as a lack of activity recorded by the monitor could reflect disability or simply a choice by an ablebodied individual to lead a sedentary life. For this reason, we chose to use self-report items from the NHANES Physical Functioning Questionnaire to create a physical 
limitation score (PLS) as an index of functional ability. The PLS was used as the outcome variable in all analyses examining the relationship between lean body mass and functioning.

As with the aforementioned models, SUDAAN's PROC REGRESS was used for all analyses of lean body mass and its relationship to physical limitation score. A Taylor Series variance estimation method for imputed data, using a "with replacement" design, was employed due to the fact that these analyses also used the DXA imputed data files. As before, NHANES two-year sample weights were applied to account for the nationally representative nature of the survey.

The data were sorted by age and gender group for NHANES subjects 60 years and over only, due to the fact that virtually no younger subjects were asked the questions chosen to create the physical limitation score while all subjects 60 years and older were interviewed. The older adults were divided into groups that most closely aligned with standard age groupings used for protein DRI ranges and were separated into the following datasets for analysis:

Age Group 1 Males: 60-70 years $(n=333)$

Age Group 1 Females: 60-70 years $(n=366)$

Age Group 2 Males: $71+$ years $(n=388)$

Age Group 2 Females: $71+$ years $(n=376)$

Participants less than 60 years of age were deleted from the dataset. More thorough descriptions of the variables used in the research models are presented in the following sections. 


\subsubsection{Response Variable}

The single response variable created and used in this series of analyses was the Physical Limitation Score (PLS), an index constructed using self-reported difficulty ratings for the performance of four basic activities of daily living. These questions were taken from the NHANES 2003-2004 Physical Functioning Questionnaire (CDC 2003), as described in Section 3.1.3 above. As mentioned previously, the four questions chosen involved difficulty to walk a quarter mile, climb ten stairs without resting, lift and carry ten pounds and stand from an armless straight chair and were those shown in published research to relate most to the ability to independently perform basic ADLs (Alexander $e t$ al. 2000; Hoeymans et al. 1996; Sayers et al. 2004). Details regarding the questionnaire interview process and the selection of questionnaire items used to create the PLS are described in Section 3.1.3.

To create the Physical Limitation Score (PLS), self-reported difficulty ratings were reassigned for each of the four tasks, as follows:

"No difficulty" = 0

"Some difficulty" = 1

"Much difficulty," "Unable," Do not do" or "Missing" = 2

Individuals with missing data were assigned a score of 2 due to the fact that these people reported being unable to walk without the use of an assistive device and were therefore not asked to rate their difficulty in walking $1 / 4$ mile or up ten stairs without resting. There were fewer than five subjects reporting "refused" or "don't know" for one or more of the four items who, as a result, were deleted from the dataset. To calculate the final PLS, the 
reassigned numerical scores were summed, resulting in a score between 0 and 8 for each of the participants, with a higher PLS indicative of greater functional limitation.

\subsubsection{Main Predictor Variables}

The main predictor variables used in this series of analyses were the five DXAderived measures of lean body mass described in Section 3.2.1.1 above:

1. Total lean body mass $(\mathrm{kg})$

2. Appendicular lean body mass $(\mathrm{kg})$

3. Muscle Mass Index $\left(\mathrm{kg} / \mathrm{m}^{2}\right)$

4. Appendicular Muscle Mass Index $\left(\mathrm{kg} / \mathrm{m}^{2}\right)$

5. Percent lean body mass $(\%)$

Five expressions of lean body mass were used to examine the unique relationship of each to physical functioning. Different from the prior set of analyses, the lean mass predictor variables used to examine the relationship between lean mass and functioning were expressed in kilograms rather than grams. This was done so that the beta value from the regression analyses would be large enough to easily interpret $(>0.000)$. Each of the main predictor variables is described in further detail in Section 3.2.1.1.

\subsubsection{Covariables}

Covariables from NHANES demographic, body measurement and health status questionnaire datasets were used in this series of regression analyses due to their hypothesized relationship to both physical limitation and lean body mass.

Body Mass Index (BMI) Group was used to control for body size and its relationship to both physical limitation and lean mass in all analyses within this series. 
By controlling for body size, an increase in physical limitation score as predicted by lean body mass would not result from merely being a larger person. BMI was grouped based on ranges found to be appropriate for older adults, as discussed in Section 2.3.1.4.1., into the following categories:

$<22.0$ (Underweight)

22.0-27.0 (Normal, referent group)

$>27.0$ (Overweight)

BMI was used categorically in this case because it does not necessarily increase or decrease together with physical limitation score. A person could theoretically report poor functioning and have a very low or a very high BMI. Used categorically, BMI still controls for size so that the relationship between physical limitation and lean body mass can be analyzed within a given BMI group. For all analyses in this series, the normal BMI range (22.0-27.0) was set as the referent group.

Age, race/ethnicity and education level were used as covariables in all analyses to control for physiological, genetic and socioeconomic differences among NHANES participants. Since participants were already divided into datasets based on age group, age was used as a quantitative variable within each. Race/ethnicity and education level were both used categorically, with non-Hispanic White and the lowest level of education set as reference levels.

General Health Status was taken from the NHANES Current Health Status Questionnaire which asked, "Would you say that your general health is: excellent, very good, good, fair or poor?" This was chosen as a categorical covariable to control for a 
person's physical health that may relate to self-reported functional ability and body composition. In all analyses, "excellent" health was used as the referent category.

Physical illness/injury status was also taken from the NHANES Current Health Status Questionnaire which asked, “Thinking about your physical health, which includes physical illness and injury, for how many days during the past 30 days was your physical health not good?" Quantitative responses ranging from 0 to 30 were used to again control for a person's physical health that may relate to self-reported functional ability.

\subsubsection{Summary of Analyses Run: Lean Body Mass as Predictive of Physical Functioning}

As with protein and lean body mass models, this series of analyses was run by age and gender group for all combinations of predictor and response variables to examine the relationship between physical limitation score and various expressions of lean body mass.

The following summarizes the analyses performed, all displayed in tabular format in the results section:

Age-gender groups:

Males and Females

- $60-70$ years

- $71+$ years

$\underline{\text { Response Variable: }}$

1. Physical Limitation Score (PLS)

$\underline{\text { Main Predictor Variables: }}$

1. Total lean mass

2. Appendicular lean mass 
3. Muscle Mass Index

4. Appendicular Muscle Mass Index

5. Percent lean mass

Total number of analyses $=20$

(4 age-gender groups x 5 predictor variables x 1 response variable)

After examination of analyses of lean body mass and its relationship to physical

functioning, as will be presented in Chapter 4 and discussed in Chapter 5, it was found that greater lean mass was not significantly predictive of functioning. As a result, planned analyses of protein intake and its direct relationship to physical functioning were not performed. 


\section{Results}

Complete statistical results from all analyses described in Chapter 3 are reported in tabular format in this chapter. For all regression analyses we chose to use a significance level of 0.05 . We considered $0.05<p$-value $<0.10$ marginally significant when discussing our results. Due to the exploratory nature of this research, it was decided that no multiple comparisons adjustment was needed for the level of significance.

\subsection{Descriptive Data}

NHANES 2003-2004 descriptive data for protein intake and DXA lean body mass measures used in our research models are displayed in Tables 4.1-4.4. In general, a decrease in total daily protein intake $(\mathrm{g})$ was seen with increasing age group in both men and women. However, protein as a percent of total energy and as a percent from animal foods remained relatively consistent between groups, demonstrating that a decrease in total energy intake rather than a shift in diet macronutrient composition may contribute to the reduced protein intake seen in older adults. In fact, the mean for protein as a percent of energy was even shown to be slightly higher in older groups than in adults ages 19-50. In general, lean and appendicular lean body mass, lean and appendicular muscle mass index and percent lean mass were all shown to decrease slightly with increasing age group. 
Table 4.1 NHANES 2003-2004 Descriptive Statistics: Protein intake in women NHANES 2003-2004: Women Percentiles

\begin{tabular}{|c|c|c|c|c|c|c|c|c|c|c|}
\hline & $\begin{array}{c}\text { Age } \\
\text { Group }\end{array}$ & $\mathbf{n}^{\mathrm{a}}$ & Mean & $\begin{array}{c}\text { SE } \\
\text { Mean }\end{array}$ & $\begin{array}{c}\text { Range } \\
\left(5^{\text {th }} \text { to } 95^{\text {th }}\right. \\
\text { percentile })\end{array}$ & $5 \%$ & $25 \%$ & $50 \%$ & $75 \%$ & $95 \%$ \\
\hline \multirow{3}{*}{$\begin{array}{l}\text { Total Protein Intake } \\
\qquad(\mathrm{g})^{\mathbf{b}}\end{array}$} & $19-50$ & 1260 & 72.86 & 1.18 & 87.83 & 34.24 & 53.17 & 69.49 & 87.15 & 122.07 \\
\hline & $51-70$ & 618 & 67.05 & 1.88 & 84.09 & 29.99 & 49.67 & 64.80 & 80.75 & 114.08 \\
\hline & $71+$ & 419 & 59.26 & 1.14 & 65.58 & 30.31 & 43.99 & 58.08 & 69.30 & 95.89 \\
\hline \multirow{3}{*}{$\begin{array}{l}\text { Protein as a Percent of } \\
\text { Total Daily Energy }\end{array}$} & $19-50$ & 1260 & 15.45 & 0.25 & 13.28 & 9.45 & 12.53 & 14.98 & 17.80 & 22.73 \\
\hline & $51-70$ & 618 & 16.13 & 0.29 & 13.30 & 10.16 & 13.21 & 15.78 & 18.24 & 23.46 \\
\hline & $71+$ & 419 & 15.71 & 0.20 & 11.91 & 10.27 & 13.38 & 15.04 & 17.64 & 22.18 \\
\hline \multirow{3}{*}{$\begin{array}{l}\text { Protein as a Percent } \\
\text { from Animal Foods }\end{array}$} & $19-50$ & 1051 & 63.45 & 0.70 & 43.85 & 38.21 & 57.07 & 65.15 & 72.85 & 82.06 \\
\hline & $51-70$ & 618 & 62.42 & 0.83 & 46.35 & 35.50 & 55.43 & 64.38 & 72.02 & 81.85 \\
\hline & $71+$ & 419 & 61.08 & 0.81 & 40.33 & 38.78 & 54.23 & 62.02 & 69.41 & 79.11 \\
\hline
\end{tabular}

${ }^{a}$ Analytic n shown; each person was weighted to account for complex survey design (including oversampling), survey non-response and post-stratification; subjects included had two days of reliable dietary intake data

${ }^{\mathrm{b}}$ Total daily protein intake $(\mathrm{g})$ is an average from two $24 \mathrm{~h}$ recalls

${ }^{\mathrm{c}}$ Protein as a $\%$ of energy is an estimate from an average of two $24 \mathrm{~h}$ recalls and $=$ (percent of energy from protein day $1+$ percent of energy from protein day 2) / 2

${ }^{\mathrm{d}}$ Protein as a \% from animal foods is an average from two 24-hour recalls and $=($ Day 1 percent of protein from animal sources + Day 2 percent of protein from animal sources) / 2; animal protein included all meat, fish, poultry, eggs and dairy. 
Table 4.2 NHANES 2003-2004 Descriptive Statistics: Protein intake in men NHANES 2003-2004: Men Percentiles

\begin{tabular}{|c|c|c|c|c|c|c|c|c|c|c|}
\hline \multicolumn{6}{|l|}{ VAALS $2003-2004$} & \multicolumn{5}{|c|}{ percentis } \\
\hline & $\begin{array}{c}\text { Age } \\
\text { group }\end{array}$ & $\mathbf{n}^{\mathrm{a}}$ & Mean & $\begin{array}{c}\text { SE } \\
\text { Mean }\end{array}$ & $\begin{array}{c}\text { Range } \\
\left(5^{\text {th }} \text { to } 95^{\text {th }}\right. \\
\text { percentile })\end{array}$ & $5 \%$ & $25 \%$ & $50 \%$ & $75 \%$ & $95 \%$ \\
\hline \multirow{3}{*}{$\begin{array}{l}\text { Total Protein Intake } \\
(\mathbf{g})^{b}\end{array}$} & $19-50$ & 1109 & 106.15 & 1.73 & 123.23 & 51.86 & 80.76 & 102.64 & 126.21 & 175.09 \\
\hline & $51-70$ & 556 & 91.07 & 1.73 & 103.14 & 46.53 & 67.37 & 87.33 & 111.35 & 149.67 \\
\hline & $71+$ & 399 & 75.85 & 2.26 & 90.43 & 34.33 & 57.07 & 75.00 & 91.78 & 124.76 \\
\hline \multirow{3}{*}{$\begin{array}{l}\text { Protein as a Percent of } \\
\text { Total Daily Energy }\end{array}$} & $19-50$ & 1108 & 15.61 & 0.20 & 11.63 & 9.99 & 13.09 & 15.13 & 17.60 & 21.62 \\
\hline & $51-70$ & 556 & 16.40 & 0.20 & 12.83 & 10.44 & 13.33 & 16.19 & 19.11 & 23.27 \\
\hline & $71+$ & 399 & 16.18 & 0.26 & 11.59 & 10.90 & 13.86 & 15.77 & 18.03 & 22.49 \\
\hline \multirow{3}{*}{$\begin{array}{l}\text { Protein as a Percent } \\
\text { from Animal Foods }\end{array}$} & $19-50$ & 1108 & 65.33 & 0.81 & 40.99 & 41.41 & 58.69 & 67.05 & 73.85 & 82.40 \\
\hline & $51-70$ & 556 & 64.69 & 0.62 & 43.48 & 39.43 & 57.44 & 66.50 & 73.35 & 82.91 \\
\hline & $71+$ & 399 & 62.56 & 1.01 & 42.57 & 38.67 & 54.80 & 63.80 & 72.02 & 81.24 \\
\hline
\end{tabular}

${ }^{a}$ Analytic n shown; each person was weighted to account for complex survey design (including oversampling), survey non-response and post-stratification; subjects included had two days of reliable dietary intake data

${ }^{\mathrm{b}}$ Total daily protein intake $(\mathrm{g})$ is an average from two $24 \mathrm{~h}$ recalls

${ }^{c}$ Protein as a $\%$ of energy is an estimate from an average of two $24 \mathrm{~h}$ recalls and $=$ (percent of energy from protein day $1+$ percent of energy from protein day 2) / 2

${ }^{\mathrm{d}}$ Protein as a \% from animal foods is an average from two 24-hour recalls and $=($ Day 1 percent of protein from animal sources + Day 2 percent of protein from animal sources) / 2; animal protein included all meat, fish, poultry, eggs and dairy. 
Table 4.3 NHANES 2003-2004 Descriptive Statistics: Lean body mass in women

\begin{tabular}{|c|c|c|c|c|c|c|c|c|c|c|}
\hline \multirow[t]{2}{*}{ NHANES 2003-2004: Women } & \multirow{2}{*}{$\begin{array}{l}\text { Age } \\
\text { Group }\end{array}$} & \multirow[t]{2}{*}{$\mathbf{n}^{\mathrm{a}}$} & \multirow[t]{2}{*}{ Mean } & \multirow{2}{*}{$\begin{array}{c}\text { SE } \\
\text { Mean }\end{array}$} & \multirow{2}{*}{$\begin{array}{c}\text { Range } \\
\left(5^{\text {th }} \text { to } 95^{\text {th }}\right. \\
\text { percentile })\end{array}$} & \multicolumn{5}{|c|}{ Percentiles } \\
\hline & & & & & & $5 \%$ & $25 \%$ & $\mathbf{5 0 \%}$ & $75 \%$ & $95 \%$ \\
\hline \multirow{3}{*}{ Total Lean Mass $(\mathbf{k g})^{b}$} & $19-50$ & 1044 & 43.01 & 0.34 & 25.22 & 32.51 & 37.37 & 41.50 & 47.20 & 57.73 \\
\hline & $51-71$ & 610 & 41.67 & 0.39 & 25.09 & 30.76 & 36.66 & 40.54 & 46.16 & 55.85 \\
\hline & $71+$ & 406 & 37.94 & 0.40 & 19.21 & 30.01 & 33.94 & 37.13 & 40.96 & 49.22 \\
\hline \multirow[t]{3}{*}{ Appendicular Lean Mass (kg) } & $19-50$ & 1044 & 18.33 & 0.16 & 12.93 & 13.19 & 15.57 & 17.56 & 20.31 & 26.12 \\
\hline & $51-70$ & 610 & 17.31 & 0.24 & 12.75 & 12.06 & 14.69 & 16.82 & 18.96 & 24.81 \\
\hline & $71+$ & 406 & 15.40 & 0.19 & 9.19 & 11.56 & 13.44 & 14.91 & 16.69 & 20.75 \\
\hline \multirow{3}{*}{$\begin{array}{l}\text { Total Muscle Mass Index } \\
\left(\mathrm{kg} / \mathrm{m}^{2}\right)^{\mathrm{d}}\end{array}$} & $19-50$ & 1037 & 16.11 & 0.12 & 8.87 & 12.56 & 14.15 & 15.52 & 17.69 & 21.43 \\
\hline & $51-70$ & 604 & 15.80 & 0.16 & 8.46 & 12.37 & 13.90 & 15.28 & 17.08 & 20.83 \\
\hline & $71+$ & 401 & 15.10 & 0.15 & 6.11 & 12.54 & 13.64 & 14.82 & 16.11 & 18.65 \\
\hline \multirow{3}{*}{$\begin{array}{l}\text { Appendicular Muscle Mass Index } \\
\qquad\left(\mathrm{kg} / \mathrm{m}^{2}\right)^{\mathrm{e}}\end{array}$} & $19-50$ & 1037 & 6.86 & 0.06 & 4.42 & 5.11 & 5.86 & 6.58 & 7.57 & 9.53 \\
\hline & $51-70$ & 604 & 6.55 & 0.09 & 4.52 & 4.79 & 5.65 & 6.29 & 7.22 & 9.31 \\
\hline & $71+$ & 401 & 6.12 & 0.08 & 3.17 & 4.85 & 5.43 & 5.99 & 6.65 & 8.02 \\
\hline \multirow[t]{3}{*}{ Percent Lean Body Mass (\%) } & $19-50$ & 1038 & 58.50 & 0.37 & 21.89 & 47.62 & 53.67 & 58.19 & 62.99 & 69.51 \\
\hline & $51-70$ & 604 & 55.61 & 0.24 & 18.42 & 46.77 & 51.79 & 55.13 & 58.90 & 65.19 \\
\hline & $71+$ & 404 & 55.62 & 0.29 & 17.55 & 48.00 & 51.92 & 55.24 & 58.22 & 65.55 \\
\hline
\end{tabular}

${ }^{\mathrm{a}}$ Analytic $\mathrm{n}$ shown; each person was weighted to account for complex survey design (including oversampling), survey non-response and post-stratification;

subjects included presented data values (imputed or measured) for specific body composition measures

${ }^{\mathrm{b}}$ Total lean body mass $(\mathrm{kg})$ is an estimate from QDR 4500 fan beam DXA scan; fat-free mass = lean muscle mass, excluding fat and bone; some DXA values are multiply imputed due to nonrandom missing data

${ }^{c}$ Appendicular lean body mass $(\mathrm{kg})$ is an estimate from QDR 4500 fan beam DXA scan; appendicular fat-free mass includes the arms, legs, hands and feet; some DXA values are multiply imputed due to nonrandom missing data

${ }^{\mathrm{d}}$ Muscle Mass Index (MMI) is calculated from QDR 4500 fan beam DXA estimates of lean muscle mass, excluding fat and bone;

$\mathrm{MMI}=($ Fat-free mass $(\mathrm{kg})) /\left(\right.$ Height $\left.\left(\mathrm{m}^{2}\right)\right)$; some DXA values are multiply imputed due to nonrandom missing data

e Appendicular Muscle Mass Index (AMMI) is calculated from QDR 4500 fan beam DXA estimates of lean muscle mass, excluding fat and

bone; $\mathrm{AMMI}=($ Fat-free mass of the arms, legs, hands and feet $(\mathrm{kg})) /\left(\operatorname{Height}\left(\mathrm{m}^{2}\right)\right)$; some DXA values are multiply imputed due to nonrandom missing data

${ }^{\mathrm{f}}$ Percent lean body mass $(\%)$ is an estimate from QDR 4500 fan beam DXA scan; fat-free mass= lean muscle mass, excluding fat and bone;

$\%$ lean $=(($ Lean body mass/Total body mass $) * 100)$; some DXA values are multiply imputed due to nonrandom missing data 
Table 4.4 NHANES 2003-2004 Descriptive Statistics: Lean body mass in men

\begin{tabular}{|c|c|c|c|c|c|c|c|c|c|c|}
\hline \multirow[t]{2}{*}{ NHANES 2003-2004: Men } & \multirow{2}{*}{$\begin{array}{c}\text { Age } \\
\text { Group }\end{array}$} & \multirow[t]{2}{*}{$\mathbf{n}^{\mathrm{a}}$} & \multirow[t]{2}{*}{ Mean } & \multirow{2}{*}{$\begin{array}{c}\text { SE } \\
\text { Mean }\end{array}$} & \multirow{2}{*}{$\begin{array}{c}\text { Range } \\
\left(5^{\text {th }} \text { to } 95^{\text {th }}\right. \\
\text { percentile })\end{array}$} & \multicolumn{5}{|c|}{ Percentiles } \\
\hline & & & & & & $5 \%$ & $25 \%$ & $\mathbf{5 0 \%}$ & $75 \%$ & $95 \%$ \\
\hline \multirow[t]{3}{*}{ Total Lean Mass $(\mathrm{kg})^{\mathrm{b}}$} & $19-50$ & 1100 & 60.97 & 0.46 & 32.36 & 46.47 & 54.43 & 59.77 & 66.42 & 78.83 \\
\hline & $51-71$ & 549 & 60.07 & 0.46 & 29.70 & 46.05 & 53.71 & 58.98 & 65.58 & 75.75 \\
\hline & $71+$ & 390 & 53.47 & 0.59 & 28.88 & 40.52 & 47.85 & 52.74 & 58.51 & 69.40 \\
\hline \multirow[t]{3}{*}{ Appendicular Lean Mass (kg) } & $19-50$ & 1100 & 27.53 & 0.23 & 16.05 & 20.40 & 24.15 & 26.92 & 30.35 & 36.45 \\
\hline & $51-70$ & 549 & 26.25 & 0.23 & 15.06 & 19.25 & 22.96 & 25.94 & 29.07 & 34.31 \\
\hline & $71+$ & 390 & 22.68 & 0.26 & 12.57 & 16.97 & 19.87 & 22.42 & 24.83 & 29.54 \\
\hline \multirow[t]{3}{*}{ Total Muscle Mass Index $\left(\mathrm{kg} / \mathrm{m}^{2}\right)^{\mathrm{d}}$} & $19-50$ & 1091 & 19.38 & 0.13 & 8.55 & 15.49 & 17.49 & 19.21 & 21.06 & 24.04 \\
\hline & $51-70$ & 548 & 19.31 & 0.11 & 8.62 & 15.18 & 17.60 & 19.22 & 20.68 & 23.80 \\
\hline & $71+$ & 387 & 17.93 & 0.14 & 7.62 & 14.48 & 16.38 & 17.70 & 19.43 & 22.10 \\
\hline \multirow{3}{*}{$\begin{array}{l}\text { Appendicular Muscle Mass Index } \\
\qquad\left(\mathrm{kg} / \mathrm{m}^{2}\right)^{\mathrm{e}}\end{array}$} & $19-50$ & 1091 & 8.75 & 0.06 & 4.13 & 6.86 & 7.82 & 8.65 & 9.52 & 10.99 \\
\hline & $51-70$ & 548 & 8.43 & 0.05 & 4.21 & 6.40 & 7.69 & 8.39 & 9.14 & 10.61 \\
\hline & $71+$ & 387 & 7.61 & 0.06 & 3.67 & 5.89 & 6.81 & 7.50 & 8.24 & 9.56 \\
\hline \multirow[t]{3}{*}{ Percent Lean Body Mass (\%) } & $19-50$ & 1092 & 70.13 & 0.16 & 19.27 & 60.52 & 66.47 & 70.14 & 73.89 & 79.79 \\
\hline & $51-70$ & 549 & 67.09 & 0.32 & 16.11 & 59.27 & 63.66 & 67.01 & 70.65 & 75.38 \\
\hline & $71+$ & 388 & 66.20 & 0.31 & 16.43 & 58.63 & 62.86 & 66.06 & 69.41 & 75.06 \\
\hline
\end{tabular}

${ }^{a}$ Analytic n shown; each person was weighted to account for complex survey design (including oversampling), survey non-response and post-stratification; subjects included presented data values (imputed or measured) for specific body composition measures

${ }^{\mathrm{b}}$ Total lean body mass $(\mathrm{kg})$ is an estimate from QDR 4500 fan beam DXA scan; fat-free mass = lean muscle mass, excluding fat and bone; some DXA values are multiply imputed due to nonrandom missing data

${ }^{c}$ Appendicular lean body mass $(\mathrm{kg})$ is an estimate from QDR 4500 fan beam DXA scan; appendicular fat-free mass includes the arms, legs,

hands and feet; some DXA values are multiply imputed due to nonrandom missing data

${ }^{\mathrm{d}}$ Muscle Mass Index (MMI) is calculated from QDR 4500 fan beam DXA estimates of lean muscle mass, excluding fat and bone;

$\mathrm{MMI}=($ Fat-free mass $(\mathrm{kg})) /\left(\operatorname{Height}_{\left.\left(\mathrm{m}^{2}\right)\right)}\right.$; some DXA values are multiply imputed due to nonrandom missing data

${ }^{\mathrm{e}}$ Appendicular Muscle Mass Index (AMMI) is calculated from QDR 4500 fan beam DXA estimates of lean muscle mass, excluding fat and bone; $\mathrm{AMMI}=($ Fat-free mass of the arms, legs, hands and feet $(\mathrm{kg})) /\left(\right.$ Height $\left.\left(\mathrm{m}^{2}\right)\right)$; some DXA values are multiply imputed due to nonrandom missing data

${ }^{\mathrm{f}}$ Percent lean body mass $(\%)$ is an estimate from QDR 4500 fan beam DXA scan; fat-free mass= lean muscle mass, excluding fat and bone;

$\%$ lean $=\left((\text { Lean body mass/Total body mass })^{*} 100\right) ;$ some DXA values are multiply imputed due to nonrandom missing data 


\subsection{Multiple Regression Analyses: Protein Intake as Predictive of Total Lean Body Mass}

Total lean body mass was not found to be significantly predicted by protein intake expressed as grams per day, a percentage of total daily energy or as a percent from animal foods in any age-gender group when an alpha of 0.05 was used (Tables 4.5-4.10).

While protein was not a significant predictor of lean mass at alpha 0.05 , total grams of protein intake was moderately, positively predictive of total lean mass in men of all ages (p-value <0.10) but not in women (Tables 4.5 and 4.6). Protein expressed as a percent of total daily energy was moderately, positively predictive of total lean body mass in men of all ages and women 51-70 years (p-value $<0.10$ ), as seen in Tables 4.7 and 4.8. Protein as a percent from animal foods was moderately, positively predictive of total lean body mass only in young men aged 19-50 (p-value <0.10, Table 4.10).

When examining the predictive value of other covariables, total energy intake showed a significant, positive relationship to total lean body mass when protein was expressed as a percent of energy and as a percent from animal foods but was not always a significant predictor in analyses where protein was expressed as total grams of daily intake. Body Mass Index (BMI) showed a highly significant positive association with total lean body mass in men and women of all ages $(\mathrm{p}$-value $<0.001)$ in all analyses, while physical activity level/intensity was not significantly associated with total lean mass in any group.

Age was significantly, negatively associated with total lean body mass in men and women $71+$ years only, while race/ethnicity was nearly always significantly associated with total lean mass. Compared to non-Hispanic Whites, being Mexican American or of other Hispanic origin was associated with a lesser lean mass at a given protein intake 
while being Non-Hispanic Black was associated with a greater lean mass. While not always significant, an overall positive trend was also seen for the relationship between total lean mass and increasing levels of education in men and women of all ages. 
Table 4.5 Total lean mass $(\mathrm{g})^{\mathrm{a}}$ as predicted by total daily protein intake $(\mathrm{g})$ in women

\begin{tabular}{|c|c|c|c|c|c|c|c|c|c|}
\hline & \multicolumn{3}{|c|}{ Women 19-50 $(n=578)^{b}$} & \multicolumn{3}{|c|}{ Women 51-70 $(n=474)^{b}$} & \multicolumn{3}{|c|}{ Women $71+(n=294)^{b}$} \\
\hline & $\beta$ & SE & $\mathrm{p}$ & $\beta$ & $\mathrm{SE}$ & $\mathrm{p}$ & $\beta$ & $\mathrm{SE}$ & $\mathrm{p}$ \\
\hline Total daily protein intake $(\mathrm{g})^{\mathrm{c}}$ & 4.211 & 10.044 & 0.683 & 17.120 & 11.871 & 0.174 & -17.101 & 9.994 & 0.111 \\
\hline 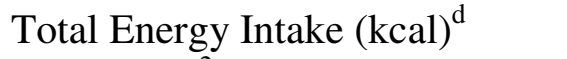 & 0.935 & 0.417 & 0.043 & 0.587 & 0.615 & 0.357 & 2.037 & 0.578 & 0.004 \\
\hline $\operatorname{BMI}\left(\mathrm{kg} / \mathrm{m}^{2}\right)$ & 913.427 & 42.774 & 0.000 & 858.780 & 33.126 & 0.000 & 810.230 & 43.120 & 0.000 \\
\hline Activity Level/Intensity ${ }^{\mathrm{e}}$ & 1.197 & 1.461 & 0.428 & 3.296 & 1.711 & 0.077 & -3.463 & 2.495 & 0.189 \\
\hline $\operatorname{Age}(y)^{\mathrm{f}}$ & -22.855 & 23.077 & 0.340 & -5.706 & 46.744 & 0.905 & -167.578 & 59.777 & 0.015 \\
\hline \multicolumn{10}{|l|}{ Race/Ethnicity } \\
\hline Non-Hispanic White (referent) & 0.000 & 0.000 & & 0.000 & 0.000 & & 0.000 & 0.000 & \\
\hline Mexican American & -2667.202 & 682.431 & 0.002 & -3119.101 & 468.256 & 0.000 & -3479.392 & 752.311 & 0.001 \\
\hline Other Hispanic & -2184.798 & 909.628 & 0.033 & -5608.436 & 878.743 & 0.000 & -2088.877 & 663.273 & 0.008 \\
\hline Non-Hispanic Black & 64.458 & 524.724 & 0.904 & 2345.289 & 548.812 & 0.001 & 3155.198 & 828.035 & 0.002 \\
\hline Other (includes multi-racial) & -2431.031 & 678.691 & 0.003 & -1622.697 & 1081.942 & 0.158 & 933.945 & 1273.446 & 0.478 \\
\hline \multicolumn{10}{|l|}{ Education } \\
\hline Less than 9th grade (referent) & 0.000 & 0.000 & & 0.000 & 0.000 & & 0.000 & 0.000 & \\
\hline 9th-11th grade & 2226.792 & 953.802 & 0.037 & -818.903 & 1133.902 & 0.483 & -701.580 & 908.322 & 0.454 \\
\hline High School Grad & 1059.106 & 889.899 & 0.257 & 383.645 & 726.309 & 0.606 & -578.261 & 802.184 & 0.484 \\
\hline Some College/AA & 1975.327 & 762.870 & 0.023 & 1559.920 & 660.194 & 0.035 & -1045.614 & 626.945 & 0.124 \\
\hline College Grad or Above & 3282.211 & 1071.524 & 0.009 & 1448.678 & 724.888 & 0.068 & 146.893 & 648.492 & 0.825 \\
\hline Multiple $\mathrm{R}^{2}$ & & 0.75 & & & 0.68 & & & 0.68 & \\
\hline
\end{tabular}

${ }^{\mathrm{a}}$ Total lean body mass ( $\mathrm{g}$ ) is an estimate from QDR 4500 fan beam DXA scan; fat-free mass = lean muscle mass, excluding fat and bone; some DXA values are multiply imputed due to nonrandom missing data

${ }^{\mathrm{b}}$ Analytic $\mathrm{n}$ shown; each person was weighted to account for complex survey design (including oversampling), survey non-response and post-stratification

${ }^{\mathrm{c}}$ Total daily protein intake $(\mathrm{g})$ is an average from two $24 \mathrm{~h}$ recalls

d Total energy intake (kcal) is an average from two $24 \mathrm{~h}$ recalls

e Activity Level/Intensity is an average intensity value from physical activity accelerometer over a 4-7 day wear period; units correspond to physical activity METS

${ }^{\mathrm{f}}$ Age is reported in years at time of health screening; individuals $>85 \mathrm{y}$ are all topcoded at 85 years of age 
Table 4.6 Total lean mass $(\mathrm{g})^{\mathrm{a}}$ as predicted by total daily protein intake $(\mathrm{g})$ in men

\begin{tabular}{|c|c|c|c|c|c|c|c|c|c|}
\hline & \multicolumn{3}{|c|}{ Men 19-50 $(n=648)^{b}$} & \multicolumn{3}{|c|}{ Men 51-70 $(n=435)^{b}$} & \multicolumn{3}{|c|}{ Men 71+ $(n=280)^{b}$} \\
\hline & $\beta$ & SE & $\mathrm{p}$ & $\beta$ & SE & $\mathrm{p}$ & $\beta$ & SE & $\mathrm{p}$ \\
\hline Total daily protein intake $(\mathrm{g})^{\mathrm{c}}$ & 20.613 & 10.627 & 0.074 & 26.434 & 14.842 & 0.098 & 53.824 & 25.370 & $\mathbf{0 . 0 5 3}$ \\
\hline Total Energy Intake $(\mathrm{kcal})^{\mathrm{d}}$ & 0.670 & 0.398 & 0.116 & 1.051 & 0.578 & 0.092 & 0.350 & 1.042 & 0.742 \\
\hline $\mathrm{BMI}\left(\mathrm{kg} / \mathrm{m}^{2}\right)$ & 1456.873 & 50.665 & 0.000 & 1382.680 & 85.764 & 0.000 & 1259.441 & 92.916 & 0.000 \\
\hline Activity Level/Intensity ${ }^{\mathrm{e}}$ & 2.849 & 1.806 & 0.138 & 0.238 & 2.775 & 0.933 & 4.621 & 3.770 & 0.244 \\
\hline $\operatorname{Age}(y)^{\mathrm{f}}$ & -23.415 & 21.576 & 0.297 & -78.578 & 43.178 & 0.092 & -289.731 & 94.396 & 0.009 \\
\hline \multicolumn{10}{|l|}{ Race/Ethnicity } \\
\hline Non-Hispanic White (referent) & 0.000 & 0.000 & & 0.000 & 0.000 & & 0.000 & 0.000 & \\
\hline Mexican American & -5406.599 & 777.125 & 0.000 & -3433.559 & 1091.977 & 0.008 & -2905.634 & 977.084 & 0.011 \\
\hline Other Hispanic & -4458.731 & 1780.603 & 0.026 & -6200.797 & 1403.110 & 0.001 & 735.724 & 1335.797 & 0.591 \\
\hline Non-Hispanic Black & 1371.527 & 645.902 & 0.053 & 3126.400 & 944.359 & 0.006 & 300.849 & 1106.332 & 0.790 \\
\hline Other (includes multi-racial) & -3355.734 & 1515.437 & 0.045 & -5858.267 & 1352.885 & 0.001 & -4039.092 & 2485.499 & 0.128 \\
\hline \multicolumn{10}{|l|}{ Education } \\
\hline Less than 9th grade (referent) & 0.000 & 0.000 & & 0.000 & 0.000 & & 0.000 & 0.000 & \\
\hline 9th-11th grade & -748.446 & 916.417 & 0.429 & -910.282 & 1502.564 & 0.555 & 1242.293 & 1314.775 & 0.362 \\
\hline High School Grad & 1350.792 & 818.725 & 0.123 & 488.104 & 1878.027 & 0.799 & 708.654 & 1434.047 & 0.629 \\
\hline Some College/AA & 2153.935 & 801.707 & 0.019 & 635.062 & 1561.843 & 0.691 & 1023.008 & 1305.618 & 0.447 \\
\hline College Grad or Above & 1805.300 & 804.204 & 0.043 & 1418.437 & 1590.820 & 0.388 & 1368.298 & 1248.566 & 0.293 \\
\hline Multiple $\mathrm{R}^{2}$ & & 0.69 & & & 0.65 & & & 0.65 & \\
\hline
\end{tabular}

${ }^{\mathrm{a}}$ Total lean body mass ( $\mathrm{g}$ ) is an estimate from QDR 4500 fan beam DXA scan; fat-free mass = lean muscle mass, excluding fat and bone; some DXA values are multiply imputed due to nonrandom missing data

${ }^{\mathrm{b}}$ Analytic $\mathrm{n}$ shown; each person was weighted to account for complex survey design (including oversampling), survey non-response and post-stratification

${ }^{\mathrm{c}}$ Total daily protein intake $(\mathrm{g})$ is an average from two $24 \mathrm{~h}$ recalls

d Total energy intake (kcal) is an average from two $24 \mathrm{~h}$ recalls

e Activity Level/Intensity is an average intensity value from physical activity accelerometer over a 4-7 day wear period; units correspond to physical activity METS

${ }^{\mathrm{f}}$ Age is reported in years at time of health screening; individuals $>85 \mathrm{y}$ are all topcoded at 85 years of age 
Table 4.7 Total lean mass $(\mathrm{g})^{\mathrm{a}}$ as predicted by protein as a percent of total daily energy $(\%)$ in women

\begin{tabular}{|c|c|c|c|c|c|c|c|c|c|}
\hline & \multicolumn{3}{|c|}{ Women 19-50 $(n=578)^{b}$} & \multicolumn{3}{|c|}{ Women 51-70 $(n=474)^{b}$} & \multicolumn{3}{|c|}{ Women $71+(n=294)^{b}$} \\
\hline & $\beta$ & SE & $\mathrm{p}$ & $\beta$ & SE & $\mathrm{p}$ & $\beta$ & SE & $\mathrm{p}$ \\
\hline Protein as \% of energy ${ }^{c}$ & 2.264 & 51.432 & 0.966 & 83.084 & 42.917 & 0.076 & -62.558 & 52.308 & 0.253 \\
\hline Total Energy Intake $(\mathrm{kcal})^{\mathrm{d}}$ & 915.054 & 42.925 & 0.000 & 1.277 & 0.292 & 0.001 & 1.387 & 0.460 & 0.010 \\
\hline $\mathrm{BMI}\left(\mathrm{kg} / \mathrm{m}^{2}\right)$ & 1.063 & 0.246 & 0.001 & 858.206 & 32.856 & 0.000 & 807.318 & 42.548 & 0.000 \\
\hline Activity Level/Intensity ${ }^{\mathrm{e}}$ & 1.180 & 1.479 & 0.440 & 3.333 & 1.714 & 0.074 & -3.494 & 2.489 & 0.185 \\
\hline $\operatorname{Age}(y)^{\mathrm{f}}$ & -23.404 & 23.151 & 0.331 & -4.180 & 46.557 & 0.930 & -168.434 & 60.151 & 0.015 \\
\hline \multicolumn{10}{|l|}{ Race/Ethnicity } \\
\hline $\begin{array}{l}\text { Non-Hispanic White } \\
\text { (referent) }\end{array}$ & 0.000 & 0.000 & & 0.000 & 0.000 & & 0.000 & 0.000 & \\
\hline Mexican American & -2651.273 & 684.415 & 0.002 & -3167.251 & 475.492 & 0.000 & -3462.070 & 738.179 & 0.000 \\
\hline Other Hispanic & -2166.141 & 916.999 & 0.035 & -5564.027 & 873.932 & 0.000 & -2104.499 & 678.910 & 0.008 \\
\hline Non-Hispanic Black & 57.399 & 528.149 & 0.915 & 2340.527 & 565.819 & 0.001 & 3178.542 & 835.393 & 0.002 \\
\hline $\begin{array}{l}\text { Other } \\
\text { (includes multi-racial) }\end{array}$ & -2415.631 & 677.101 & 0.003 & -1591.724 & 1079.172 & 0.165 & 934.641 & 1290.498 & 0.483 \\
\hline \multicolumn{10}{|l|}{ Education } \\
\hline Less than 9th grade (referent) & 0.000 & 0.000 & & 0.000 & 0.000 & & 0.000 & 0.000 & \\
\hline 9th-11th grade & 2260.552 & 966.037 & 0.037 & -813.238 & 1145.423 & 0.490 & -691.685 & 894.478 & 0.453 \\
\hline High School Grad & 1074.825 & 888.285 & 0.249 & 420.783 & 742.569 & 0.580 & -590.684 & 802.019 & 0.475 \\
\hline Some College/AA & 2003.279 & 773.026 & 0.023 & 1570.035 & 669.261 & 0.036 & -1050.215 & 624.691 & 0.121 \\
\hline College Grad or Above & 3323.629 & 1092.818 & 0.010 & 1460.035 & 728.917 & 0.067 & 137.857 & 633.919 & 0.832 \\
\hline Multiple $\mathrm{R}^{2}$ & & 0.75 & & & 0.68 & & & 0.68 & \\
\hline
\end{tabular}

${ }^{\mathrm{a}}$ Total lean body mass (g) is an estimate from QDR 4500 fan beam DXA scan; fat-free mass= lean muscle mass, excluding fat and bone; some DXA values are multiply imputed due to nonrandom missing data

${ }^{\mathrm{b}}$ Analytic $\mathrm{n}$ shown; each person was weighted to account for complex survey design (including oversampling), survey non-response and post-stratification

${ }^{c}$ Protein as a $\%$ of energy is an estimate from an average of two $24 \mathrm{~h}$ recalls and $=(\%$ of energy from protein day $1+\%$ of energy from protein day 2$) / 2$

${ }^{\mathrm{d}}$ Total energy intake (kcal) is an average from two $24 \mathrm{~h}$ recalls

e Activity Level/Intensity is an average intensity value from a physical activity accelerometer over a 4-7 day wear period; units correspond to activity METS

${ }^{\mathrm{f}}$ Age is reported in years at time of health screening; individuals $>85 \mathrm{y}$ are all topcoded at 85 years of age 
Table 4.8 Total lean mass $(\mathrm{g})^{\mathrm{a}}$ as predicted by protein as a percent of total daily energy $(\%)$ in men

\begin{tabular}{|c|c|c|c|c|c|c|c|c|c|}
\hline & \multicolumn{3}{|c|}{ Men 19-50 $(n=648)^{b}$} & \multicolumn{3}{|c|}{ Men 51-70 $(n=435)^{b}$} & \multicolumn{3}{|c|}{ Men 71+ $(n=280)^{b}$} \\
\hline & $\beta$ & SE & $\mathrm{p}$ & $\beta$ & SE & $\mathrm{p}$ & $\beta$ & SE & $\mathrm{p}$ \\
\hline Protein as \% of energy $^{c}$ & 131.123 & 65.905 & 0.068 & 144.730 & 77.307 & 0.084 & 256.130 & 137.041 & 0.084 \\
\hline Total Energy Intake $(\mathrm{kcal})^{\mathrm{d}}$ & 1.429 & 0.344 & 0.001 & 2.058 & 0.477 & 0.001 & 2.352 & 0.391 & 0.000 \\
\hline $\mathrm{BMI}\left(\mathrm{kg} / \mathrm{m}^{2}\right)$ & 1458.494 & 50.801 & 0.000 & 1374.991 & 85.159 & 0.000 & 1255.716 & 90.219 & 0.000 \\
\hline Activity Level/Intensity ${ }^{\mathrm{e}}$ & 2.871 & 1.816 & 0.138 & 0.202 & 2.844 & 0.944 & 5.040 & 3.858 & 0.215 \\
\hline $\operatorname{Age}(y)^{\mathrm{f}}$ & -22.779 & 20.894 & 0.295 & -77.003 & 43.007 & 0.096 & -290.776 & 89.321 & 0.006 \\
\hline \multicolumn{10}{|l|}{ Race/Ethnicity } \\
\hline $\begin{array}{l}\text { Non-Hispanic White } \\
\text { (referent) }\end{array}$ & 0.000 & 0.000 & & 0.000 & 0.000 & & 0.000 & 0.000 & \\
\hline Mexican American & -5396.930 & 765.445 & 0.000 & -3371.432 & 1108.596 & 0.009 & -2923.964 & 1031.464 & 0.014 \\
\hline Other Hispanic & -4443.058 & 1774.742 & 0.026 & -6437.262 & 1276.070 & 0.000 & 714.455 & 1365.998 & 0.610 \\
\hline Non-Hispanic Black & 1353.424 & 654.525 & 0.059 & 3129.793 & 959.717 & 0.006 & 339.943 & 1057.042 & 0.753 \\
\hline $\begin{array}{l}\text { Other } \\
\text { (includes multi-racial) }\end{array}$ & -3269.142 & 1553.355 & 0.055 & -5839.973 & 1349.578 & 0.001 & -3726.299 & 2442.985 & 0.151 \\
\hline \multicolumn{10}{|l|}{ Education } \\
\hline Less than 9th grade (referent) & 0.000 & 0.000 & & 0.000 & 0.000 & & 0.000 & 0.000 & \\
\hline 9th-11th grade & -777.277 & 936.400 & 0.421 & -946.234 & 1516.772 & 0.543 & 1227.243 & 1267.407 & 0.350 \\
\hline High School Grad & 1347.025 & 814.675 & 0.122 & 429.426 & 1870.438 & 0.822 & 722.658 & 1458.922 & 0.628 \\
\hline Some College/AA & 2128.193 & 794.925 & 0.019 & 628.601 & 1569.122 & 0.695 & 1003.832 & 1299.448 & 0.453 \\
\hline College Grad or Above & 1800.953 & 801.442 & 0.042 & 1338.154 & 1614.885 & 0.422 & 1407.439 & 1289.291 & 0.295 \\
\hline Multiple $\mathrm{R}^{2}$ & & 0.69 & & & 0.64 & & & 0.65 & \\
\hline
\end{tabular}

${ }^{\mathrm{a}}$ Total lean body mass (g) is an estimate from QDR 4500 fan beam DXA scan; fat-free mass= lean muscle mass, excluding fat and bone; some DXA values are multiply imputed due to nonrandom missing data

${ }^{\mathrm{b}}$ Analytic $\mathrm{n}$ shown; each person was weighted to account for complex survey design (including oversampling), survey non-response and post-stratification

${ }^{c}$ Protein as a $\%$ of energy is an estimate from an average of two $24 \mathrm{~h}$ recalls and $=(\%$ of energy from protein day $1+\%$ of energy from protein day 2$) / 2$

${ }^{\mathrm{d}}$ Total energy intake (kcal) is an average from two $24 \mathrm{~h}$ recalls

e Activity Level/Intensity is an average intensity value from a physical activity accelerometer over a 4-7 day wear period; units correspond to activity METS

${ }^{\mathrm{f}}$ Age is reported in years at time of health screening; individuals $>85 \mathrm{y}$ are all topcoded at 85 years of age 
Table 4.9 Total lean mass $(\mathrm{g})^{\mathrm{a}}$ as predicted by protein as a percent contributed by animal foods (\%) in women

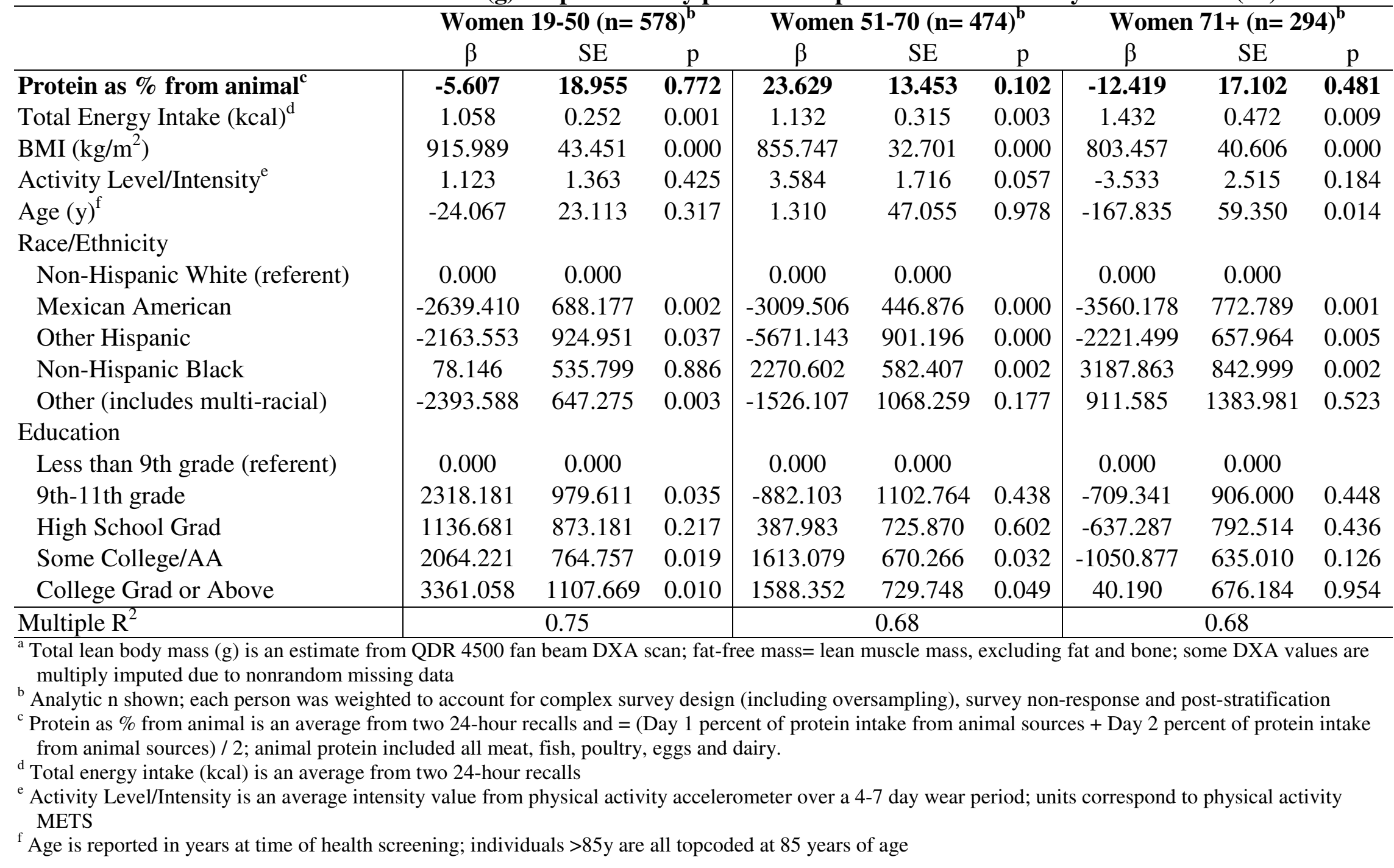


Table 4.10 Total lean mass $(\mathrm{g})^{\mathrm{a}}$ as predicted by protein as a percent contributed by animal foods $(\%)$ in men

\begin{tabular}{|c|c|c|c|c|c|c|c|c|c|}
\hline & \multicolumn{3}{|c|}{ Men 19-50 $(n=648)^{b}$} & \multicolumn{3}{|c|}{ Men 51-70 $(n=435)^{b}$} & \multicolumn{3}{|c|}{ Men 71+ $(n=280)^{b}$} \\
\hline & $\beta$ & SE & $\mathrm{p}$ & $\beta$ & SE & $\mathrm{p}$ & $\beta$ & SE & $\mathrm{p}$ \\
\hline Protein as \% from animal $^{\mathrm{c}}$ & 37.455 & 19.203 & 0.073 & -14.474 & 25.363 & 0.578 & 3.349 & 20.298 & 0.871 \\
\hline Total Energy Intake $(\mathrm{kcal})^{\mathrm{d}}$ & 1.286 & 0.319 & 0.001 & 1.847 & 0.410 & 0.001 & 1.983 & 0.469 & 0.001 \\
\hline $\mathrm{BMI}\left(\mathrm{kg} / \mathrm{m}^{2}\right)$ & 1455.623 & 50.872 & 0.000 & 1393.229 & 86.635 & 0.000 & 1252.084 & 80.486 & 0.000 \\
\hline Activity Level/Intensity ${ }^{\mathrm{e}}$ & 2.978 & 1.853 & 0.132 & 0.083 & 2.793 & 0.977 & 4.466 & 3.661 & 0.246 \\
\hline $\operatorname{Age}(y)^{\mathrm{f}}$ & -21.232 & 20.302 & 0.315 & -85.680 & 43.838 & 0.072 & -333.324 & 85.247 & 0.002 \\
\hline \multicolumn{10}{|l|}{ Race/Ethnicity } \\
\hline Non-Hispanic White (referent) & 0.000 & 0.000 & & 0.000 & 0.000 & & 0.000 & 0.000 & \\
\hline Mexican American & -5265.472 & 777.516 & 0.000 & -3510.091 & 1127.628 & 0.008 & -2672.120 & 847.297 & 0.007 \\
\hline Other Hispanic & -4493.906 & 1869.660 & 0.031 & -6157.894 & 1240.370 & 0.000 & 584.229 & 1295.430 & 0.659 \\
\hline Non-Hispanic Black & 1185.472 & 678.519 & 0.104 & 3315.745 & 979.842 & 0.005 & -251.381 & 1165.588 & 0.833 \\
\hline Other (includes multi-racial) & -3364.819 & 1613.625 & 0.057 & -6013.836 & 1395.085 & 0.001 & -3860.458 & 2205.884 & 0.103 \\
\hline \multicolumn{10}{|l|}{ Education } \\
\hline Less than 9th grade (referent) & 0.000 & 0.000 & & 0.000 & 0.000 & & 0.000 & 0.000 & \\
\hline 9th-11th grade & -824.515 & 971.773 & 0.411 & -1200.664 & 1536.914 & 0.448 & 1365.380 & 1400.907 & 0.347 \\
\hline High School Grad & 1360.988 & 820.095 & 0.121 & 333.780 & 1857.360 & 0.860 & 798.198 & 1428.904 & 0.586 \\
\hline Some College/AA & 2060.513 & 800.217 & 0.023 & 599.560 & 1641.375 & 0.721 & 1286.384 & 1206.420 & 0.305 \\
\hline College Grad or Above & 1928.224 & 808.247 & 0.033 & 1365.563 & 1563.496 & 0.398 & 1690.491 & 1140.835 & 0.162 \\
\hline Multiple $\mathrm{R}^{2}$ & & 0.69 & & & 0.64 & & & 0.64 & \\
\hline
\end{tabular}

${ }^{\mathrm{a}}$ Total lean body mass (g) is an estimate from QDR 4500 fan beam DXA scan; fat-free mass= lean muscle mass, excluding fat and bone; some DXA values are multiply imputed due to nonrandom missing data

${ }^{\mathrm{b}}$ Analytic $\mathrm{n}$ shown; each person was weighted to account for complex survey design (including oversampling), survey non-response and post-stratification

${ }^{\mathrm{c}}$ Protein as $\%$ from animal is an average from two 24 -hour recalls and = (Day 1 percent of protein intake from animal sources + Day 2 percent of protein intake from animal sources) / 2; animal protein included all meat, fish, poultry, eggs and dairy.

d Total energy intake (kcal) is an average from two 24-hour recalls

e Activity Level/Intensity is an average intensity value from physical activity accelerometer over a 4-7 day wear period; units correspond to physical activity METS

${ }^{\mathrm{f}}$ Age is reported in years at time of health screening; individuals $>85 \mathrm{y}$ are all topcoded at 85 years of age 


\subsection{Multiple Regression Analyses: Protein Intake as Predictive of Appendicular Lean Body Mass}

Appendicular lean body mass was found to be significantly, positively predicted by protein as a percent of total daily energy in men ages 19-50 (p-value 0.048). However, in all other age-gender groups, appendicular lean mass was not significantly predicted by protein intake expressed as grams per day, a percentage of total daily energy or as a percent from animal foods when an alpha of 0.05 was used (Tables 4.11-4.16).

While not significant at alpha 0.05 , total grams of protein intake moderately, positively predicted appendicular lean mass in men ages 19-50 and 51-70, as seen in Tables 4.11 and 4.12 (p-value 0.058). Protein as a percent of total energy moderately, positively predicted appendicular lean mass in men and women aged 51-70 years (pvalue $<0.10$, tables 4.13 and 4.14). Protein as a percent contributed by animal foods was not found to be a significant predictor of appendicular lean mass in any age-gender group (Tables 4.15 and 4.16).

As was the case with total lean mass, total energy intake showed a significant, positive relationship to appendicular lean mass when protein was expressed as a percent of energy and as a percent from animal foods but was not always significantly associated when protein was expressed as total grams of intake. Body Mass Index (BMI) showed a very strong positive association with appendicular lean body mass in men and women of all ages (p-value $<0.001$ ), while physical activity level/intensity was sporadically associated with appendicular lean mass across age-gender groups.

Age was significantly, negatively associated with appendicular lean body mass in all age-gender groups with the exception of women ages 51-70. As seen with total lean, 
race/ethnicity was nearly always significantly associated with appendicular lean mass, as Mexican Americans and other Hispanics demonstrated a lesser appendicular lean mass at a given protein intake compared with non-Hispanic Whites while Non-Hispanic Blacks demonstrated a greater appendicular lean mass compared with non-Hispanic Whites. While not always significant, an overall positive trend was seen for the relationship between increasing levels of education and appendicular lean mass in men and women of all ages. 
Table 4.11 Appendicular lean mass $(\mathrm{g})^{\mathrm{a}}$ as predicted by total daily protein intake $(\mathrm{g})$ in women

\begin{tabular}{|c|c|c|c|c|c|c|c|c|c|}
\hline & \multicolumn{3}{|c|}{ Women 19-50 $(n=578)^{b}$} & \multicolumn{3}{|c|}{ Women 51-70 $(n=474)^{b}$} & \multicolumn{3}{|c|}{ Women $71+(n=294)^{b}$} \\
\hline & $\beta$ & SE & $\mathrm{p}$ & $\beta$ & SE & $\mathrm{p}$ & $\beta$ & SE & $\mathrm{p}$ \\
\hline Total daily protein intake $(\mathrm{g})^{\mathrm{c}}$ & 2.474 & 5.558 & 0.666 & 10.520 & 6.167 & 0.114 & -6.731 & 5.938 & 0.277 \\
\hline Total Energy Intake (kcal) ${ }^{\mathrm{d}}$ & 0.393 & 0.220 & 0.098 & 0.209 & 0.296 & 0.494 & 0.868 & 0.301 & 0.013 \\
\hline $\operatorname{BMI}\left(\mathrm{kg} / \mathrm{m}^{2}\right)$ & 455.338 & 22.820 & 0.000 & 417.763 & 18.970 & 0.000 & 399.140 & 27.936 & 0.000 \\
\hline Activity Level/Intensity ${ }^{\mathrm{e}}$ & 1.372 & 0.725 & 0.082 & 2.308 & 0.974 & 0.035 & -0.744 & 1.344 & 0.591 \\
\hline Age $(y)^{\mathrm{f}}$ & -30.030 & 11.207 & 0.020 & -2.405 & 18.921 & 0.901 & -60.903 & 25.035 & 0.030 \\
\hline \multicolumn{10}{|l|}{ Race/Ethnicity } \\
\hline Non-Hispanic White (referent) & 0.000 & 0.000 & & 0.000 & 0.000 & & 0.000 & 0.000 & \\
\hline Mexican American & -1527.764 & 355.426 & 0.001 & -1763.553 & 281.589 & 0.000 & -1869.508 & 338.462 & 0.000 \\
\hline Other Hispanic & -931.192 & 367.740 & 0.029 & -2246.842 & 519.431 & 0.002 & -1113.691 & 159.108 & 0.000 \\
\hline Non-Hispanic Black & 1130.877 & 255.553 & 0.001 & 2226.578 & 226.112 & 0.000 & 2672.735 & 505.919 & 0.000 \\
\hline Other (includes multi-racial) & -894.950 & 318.916 & 0.015 & -432.102 & 505.112 & 0.410 & 730.248 & 541.086 & 0.210 \\
\hline \multicolumn{10}{|l|}{ Education } \\
\hline Less than 9th grade (referent) & 0.000 & 0.000 & & 0.000 & 0.000 & & 0.000 & 0.000 & \\
\hline 9th-11th grade & 1173.561 & 383.325 & 0.010 & -305.103 & 555.530 & 0.592 & -515.212 & 436.395 & 0.261 \\
\hline High School Grad & 506.575 & 393.611 & 0.223 & 562.426 & 330.828 & 0.113 & -246.255 & 343.442 & 0.486 \\
\hline Some College/AA & 1185.132 & 296.467 & 0.002 & 1251.273 & 344.258 & 0.003 & -626.436 & 294.648 & 0.060 \\
\hline College Grad or Above & 1828.207 & 519.846 & 0.004 & 1225.334 & 330.748 & 0.003 & 117.125 & 353.895 & 0.747 \\
\hline Multiple $\mathrm{R}^{2}$ & & 0.75 & & & 0.68 & & & 0.66 & \\
\hline
\end{tabular}

${ }^{a}$ Appendicular lean body mass (g) is an estimate from QDR 4500 fan beam DXA scan; appendicular fat-free mass includes the arms, legs, hands and feet; some DXA values are multiply imputed due to nonrandom missing data

${ }^{\mathrm{b}}$ Analytic $\mathrm{n}$ shown; each person was weighted to account for complex survey design (including oversampling), survey non-response and post-stratification

${ }^{\mathrm{c}}$ Total daily grams of protein intake is an average from two 24-hour recalls

d Total energy intake (kcal) is an average from two 24-hour recalls

${ }^{\mathrm{e}}$ Activity Level/Intensity is an average intensity value from physical activity accelerometer over a 4-7 day wear period; units correspond to physical activity METS

${ }^{\mathrm{f}}$ Age is reported in years at time of health screening; individuals $>85 \mathrm{y}$ are all topcoded at 85 years of age 
Table 4.12 Appendicular lean mass $(\mathrm{g})^{\mathrm{a}}$ as predicted by total daily protein intake (g) in men

\begin{tabular}{|c|c|c|c|c|c|c|c|c|c|}
\hline & \multicolumn{3}{|c|}{ Men 19-50 $(n=648)^{b}$} & \multicolumn{3}{|c|}{$\operatorname{Men} 51-70(n=435)^{b}$} & \multicolumn{3}{|c|}{ Men 71+ $(n=280)^{b}$} \\
\hline & $\beta$ & SE & $\mathrm{p}$ & $\beta$ & $\mathrm{SE}$ & $\mathrm{p}$ & $\beta$ & $\mathrm{SE}$ & $\mathrm{p}$ \\
\hline Total daily protein intake $(\mathrm{g})^{\mathrm{c}}$ & 11.636 & 5.586 & 0.058 & 18.148 & 8.740 & 0.058 & 22.505 & 13.611 & 0.122 \\
\hline 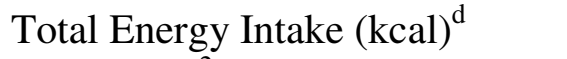 & 0.310 & 0.216 & 0.176 & 0.288 & 0.346 & 0.421 & 0.206 & 0.488 & 0.680 \\
\hline $\mathrm{BMI}\left(\mathrm{kg} / \mathrm{m}^{2}\right)$ & 668.739 & 30.614 & 0.000 & 620.979 & 44.767 & 0.000 & 557.217 & 52.291 & 0.000 \\
\hline Activity Level/Intensity ${ }^{\mathrm{e}}$ & 1.886 & 0.689 & 0.017 & 1.750 & 1.616 & 0.298 & 3.924 & 1.826 & 0.052 \\
\hline $\operatorname{Age}(y)^{f}$ & -41.669 & 11.879 & 0.004 & -72.319 & 22.426 & 0.007 & -126.582 & 46.221 & 0.017 \\
\hline \multicolumn{10}{|l|}{ Race/Ethnicity } \\
\hline Non-Hispanic White (referent) & 0.000 & 0.000 & & 0.000 & 0.000 & & 0.000 & 0.000 & \\
\hline Mexican American & -2521.376 & 390.006 & 0.000 & -1672.405 & 590.329 & 0.014 & -1619.183 & 437.392 & 0.003 \\
\hline Other Hispanic & -2057.439 & 966.588 & 0.053 & -2716.144 & 666.354 & 0.001 & 641.824 & 681.335 & 0.363 \\
\hline Non-Hispanic Black & 2529.393 & 391.094 & 0.000 & 3138.193 & 469.298 & 0.000 & 1477.879 & 661.191 & 0.043 \\
\hline Other (includes multi-racial) & -1327.373 & 632.964 & 0.056 & -2384.581 & 604.538 & 0.002 & -2646.293 & 1229.831 & 0.050 \\
\hline \multicolumn{10}{|l|}{ Education } \\
\hline Less than 9th grade (referent) & 0.000 & 0.000 & & 0.000 & 0.000 & & 0.000 & 0.000 & \\
\hline 9th-11th grade & -200.925 & 528.900 & 0.710 & -257.310 & 810.087 & 0.756 & 694.664 & 570.979 & 0.245 \\
\hline High School Grad & 793.029 & 450.861 & 0.103 & 599.758 & 916.315 & 0.524 & 348.599 & 687.350 & 0.620 \\
\hline Some College/AA & 1472.705 & 459.362 & 0.007 & 529.351 & 840.068 & 0.539 & 394.490 & 652.971 & 0.556 \\
\hline College Grad or Above & 1370.883 & 423.050 & 0.006 & 1251.779 & 835.572 & 0.158 & 556.948 & 583.022 & 0.358 \\
\hline Multiple $\mathrm{R}^{2}$ & & 0.64 & & & 0.58 & & & 0.59 & \\
\hline
\end{tabular}

${ }^{a}$ Appendicular lean body mass ( $\mathrm{g}$ ) is an estimate from QDR 4500 fan beam DXA scan; appendicular fat-free mass includes the arms, legs, hands and feet; some DXA values are multiply imputed due to nonrandom missing data

${ }^{b}$ Analytic $\mathrm{n}$ shown; each person was weighted to account for complex survey design (including oversampling), survey non-response and post-stratification

c Total daily grams of protein intake is an average from two 24-hour recalls

d Total energy intake (kcal) is an average from two 24-hour recalls

${ }^{\mathrm{e}}$ Activity Level/Intensity is an average intensity value from physical activity accelerometer over a 4-7 day wear period; units correspond to physical activity METS

${ }^{\mathrm{f}}$ Age is reported in years at time of health screening; individuals $>85 \mathrm{y}$ are all topcoded at 85 years of age 
Table 4.13 Appendicular lean mass $(\mathrm{g})^{\mathrm{a}}$ as predicted by protein as a percent of total daily energy $(\%)$ in women

\begin{tabular}{|c|c|c|c|c|c|c|c|c|c|}
\hline & \multicolumn{3}{|c|}{ Women 19-50 $(n=578)^{b}$} & \multicolumn{3}{|c|}{ Women 51-70 $(n=474)^{b}$} & \multicolumn{3}{|c|}{ Women $71+(n=294)^{b}$} \\
\hline & $\beta$ & SE & $\mathrm{p}$ & $\beta$ & SE & $\mathrm{p}$ & $\beta$ & SE & $\mathrm{p}$ \\
\hline Protein as \% of energy ${ }^{c}$ & 4.984 & 27.164 & 0.858 & 40.337 & 22.285 & 0.095 & -24.250 & 27.096 & 0.387 \\
\hline 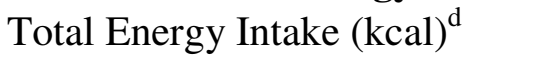 & 0.475 & 0.108 & 0.002 & 0.615 & 0.160 & 0.002 & 0.612 & 0.248 & 0.028 \\
\hline $\mathrm{BMI}\left(\mathrm{kg} / \mathrm{m}^{2}\right)$ & 455.953 & 22.787 & 0.000 & 418.011 & 19.191 & 0.000 & 397.949 & 27.587 & 0.000 \\
\hline Activity Level/Intensity ${ }^{\mathrm{e}}$ & 1.362 & 0.732 & 0.086 & 2.364 & 0.982 & 0.032 & -0.755 & 1.354 & 0.588 \\
\hline $\operatorname{Age}(y)^{f}$ & -30.167 & 11.123 & 0.018 & -1.308 & 18.714 & 0.945 & -61.234 & 25.340 & 0.031 \\
\hline \multicolumn{10}{|l|}{ Race/Ethnicity } \\
\hline Non-Hispanic White (referent) & 0.000 & 0.000 & & 0.000 & 0.000 & & 0.000 & 0.000 & \\
\hline Mexican American & -1521.036 & 356.233 & 0.001 & -1769.867 & 282.418 & 0.000 & -1863.214 & 330.567 & 0.000 \\
\hline Other Hispanic & -924.965 & 374.486 & 0.032 & -2233.798 & 522.088 & 0.002 & -1120.333 & 145.707 & 0.000 \\
\hline Non-Hispanic Black & 1127.648 & 256.423 & 0.001 & 2222.827 & 232.287 & 0.000 & 2681.947 & 507.955 & 0.000 \\
\hline Other (includes multi-racial) & -887.801 & 320.113 & 0.016 & -412.820 & 507.658 & 0.433 & 730.321 & 540.424 & 0.210 \\
\hline \multicolumn{10}{|l|}{ Education } \\
\hline Less than 9th grade (referent) & 0.000 & 0.000 & & 0.000 & 0.000 & & 0.000 & 0.000 & \\
\hline 9th-11th grade & 1188.736 & 392.605 & 0.010 & -299.602 & 559.802 & 0.601 & -511.491 & 431.371 & 0.259 \\
\hline High School Grad & 513.278 & 392.732 & 0.216 & 582.545 & 335.368 & 0.106 & -251.598 & 343.562 & 0.477 \\
\hline Some College/AA & 1195.988 & 298.950 & 0.002 & 1273.155 & 340.063 & 0.003 & -628.220 & 294.686 & 0.059 \\
\hline College Grad or Above & 1845.895 & 531.627 & 0.004 & 1252.574 & 326.112 & 0.002 & 112.815 & 351.427 & 0.755 \\
\hline Multiple $\mathrm{R}^{2}$ & & 0.75 & & & 0.68 & & & 0.67 & \\
\hline
\end{tabular}

${ }^{\mathrm{a}}$ Appendicular lean body mass ( $\mathrm{g}$ ) is an estimate from QDR 4500 fan beam DXA scan of lean muscle mass, excluding fat and bone; appendicular fat-free mass includes the arms, legs, hands and feet; some DXA values are multiply imputed due to nonrandom missing data

${ }^{\mathrm{b}}$ Analytic $\mathrm{n}$ shown; each person was weighted to account for complex survey design (including oversampling), survey non-response and post-stratification

${ }^{\mathrm{c}}$ Protein as $\%$ of energy is an average from two 24 -hour recalls and $=$ (percent of energy from protein day $1+$ percent of energy from protein day 2 ) $/ 2$

${ }^{\mathrm{d}}$ Total energy intake (kcal) is an average from two 24-hour recalls

${ }^{\mathrm{e}}$ Activity Level/Intensity is an average intensity value from physical activity accelerometer over a 4-7 day wear period; units correspond to physical activity METS

${ }^{\mathrm{f}}$ Age is reported in years at time of health screening; individuals $>85 \mathrm{y}$ are all topcoded at 85 years of age 
Table 4.14 Appendicular lean mass $(\mathrm{g})^{\mathrm{a}}$ as predicted by protein as a percent of total daily energy $(\%)$ in men

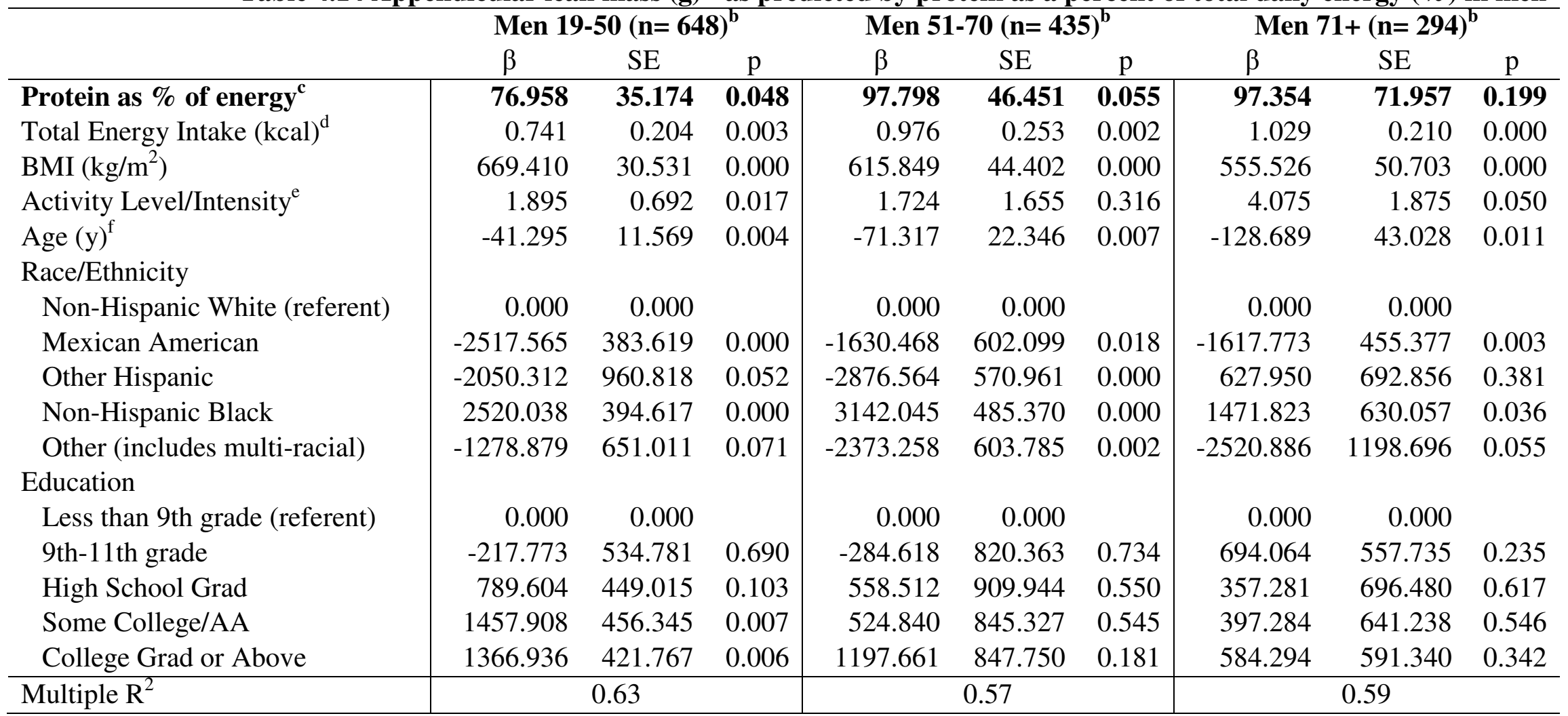

${ }^{\mathrm{a}}$ Appendicular lean body mass (g) is an estimate from QDR 4500 fan beam DXA scan of lean muscle mass, excluding fat and bone; appendicular fat-free mass includes the arms, legs, hands and feet; some DXA values are multiply imputed due to nonrandom missing data

${ }^{\mathrm{b}}$ Analytic $\mathrm{n}$ shown; each person was weighted to account for complex survey design (including oversampling), survey non-response and post-stratification

${ }^{\mathrm{c}}$ Protein as $\%$ of energy is an average from two 24 -hour recalls and $=$ (percent of energy from protein day $1+$ percent of energy from protein day 2 ) $/ 2$

${ }^{\mathrm{d}}$ Total energy intake (kcal) is an average from two 24-hour recalls

${ }^{\mathrm{e}}$ Activity Level/Intensity is an average intensity value from physical activity accelerometer over a 4-7 day wear period; units correspond to physical activity METS

${ }^{\mathrm{f}}$ Age is reported in years at time of health screening; individuals $>85 \mathrm{y}$ are all topcoded at 85 years of age 
Table 4.15 Appendicular lean mass $(\mathrm{g})^{\mathrm{a}}$ as predicted by protein as a percent contributed by animal foods (\%) in women

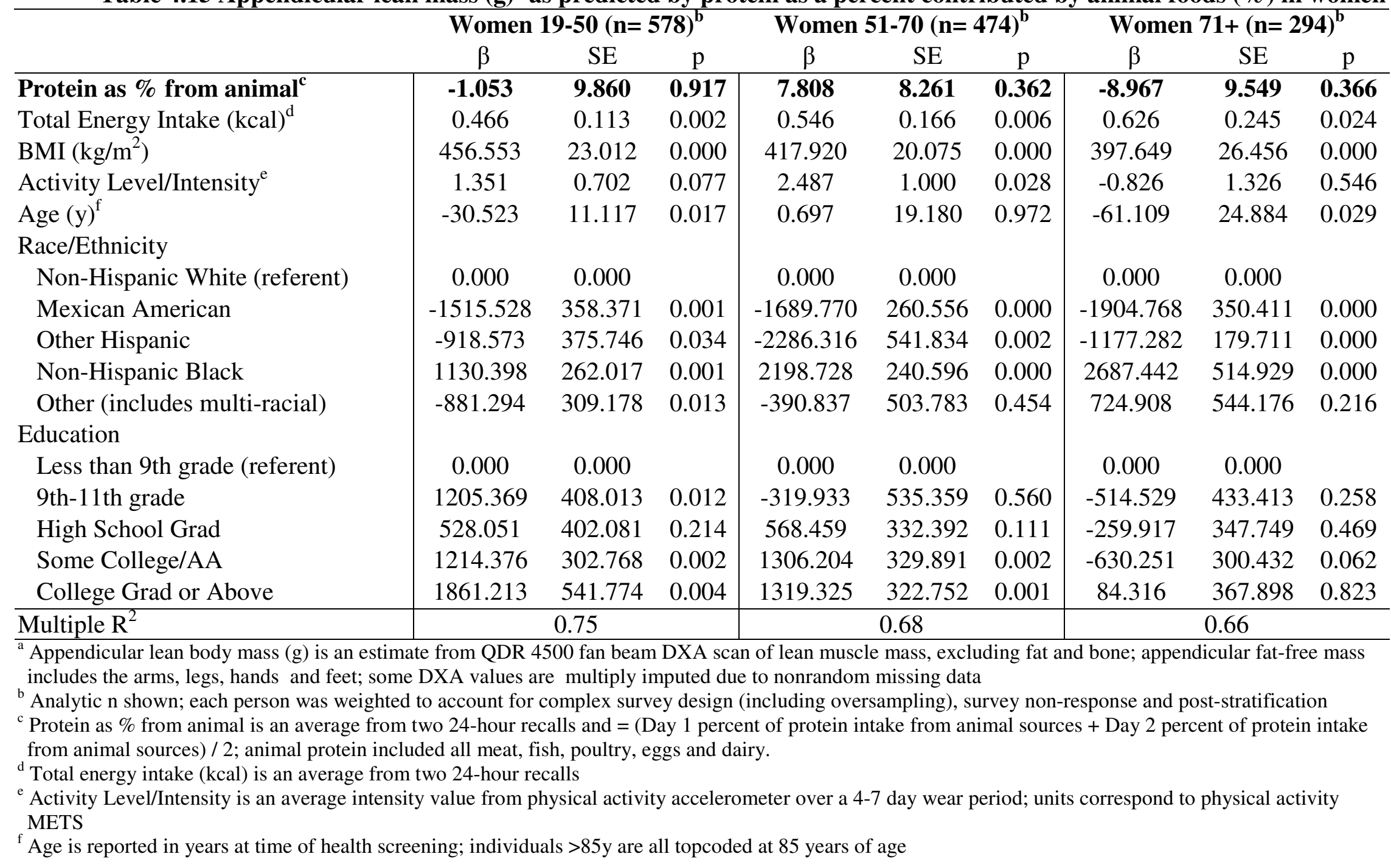


Table 4.16 Appendicular lean mass $(\mathrm{g})^{\mathrm{a}}$ as predicted by protein as a percent contributed by animal foods $(\%)$ in men

\begin{tabular}{|c|c|c|c|c|c|c|c|c|c|}
\hline & \multicolumn{3}{|c|}{ Men 19-50 $(n=648)^{b}$} & \multicolumn{3}{|c|}{ Men 51-70 $(n=435)^{b}$} & \multicolumn{3}{|c|}{ Men 71+ $(n=280)^{b}$} \\
\hline & $\beta$ & SE & $\mathrm{p}$ & $\beta$ & SE & $\mathrm{p}$ & $\beta$ & SE & $\mathrm{p}$ \\
\hline Protein as \% from animal $^{\mathrm{c}}$ & $\mathbf{1 7 . 3 6 3}$ & 10.320 & 0.116 & 0.171 & 14.190 & 0.991 & 3.845 & 11.703 & 0.748 \\
\hline Total Energy Intake (kcal) ${ }^{\mathrm{d}}$ & 0.660 & 0.189 & 0.004 & 0.834 & 0.229 & 0.003 & 0.886 & 0.217 & 0.001 \\
\hline $\mathrm{BMI}\left(\mathrm{kg} / \mathrm{m}^{2}\right)$ & 669.420 & 31.028 & 0.000 & 625.066 & 45.734 & 0.000 & 554.062 & 47.044 & 0.000 \\
\hline Activity Level/Intensity ${ }^{\mathrm{e}}$ & 1.959 & 0.713 & 0.017 & 1.664 & 1.631 & 0.326 & 3.915 & 1.858 & 0.057 \\
\hline $\operatorname{Age}(y)^{\mathrm{f}}$ & -40.662 & 11.297 & 0.003 & -76.246 & 23.035 & 0.006 & -143.799 & 38.337 & 0.003 \\
\hline \multicolumn{10}{|l|}{ Race/Ethnicity } \\
\hline Non-Hispanic White (referent) & 0.000 & 0.000 & & 0.000 & 0.000 & & 0.000 & 0.000 & \\
\hline Mexican American & -2447.573 & 384.876 & 0.000 & -1674.309 & 615.913 & 0.018 & -1511.940 & 386.476 & 0.002 \\
\hline Other Hispanic & -2064.429 & 1023.633 & 0.064 & -2757.649 & 562.934 & 0.000 & 579.057 & 664.170 & 0.399 \\
\hline Non-Hispanic Black & 2437.436 & 402.783 & 0.000 & 3236.854 & 486.329 & 0.000 & 1245.451 & 681.851 & 0.090 \\
\hline Other (includes multi-racial) & -1321.109 & 673.137 & 0.071 & -2449.897 & 632.106 & 0.002 & -2566.314 & 1107.117 & 0.037 \\
\hline \multicolumn{10}{|l|}{ Education } \\
\hline Less than 9th grade (referent) & 0.000 & 0.000 & & 0.000 & 0.000 & & 0.000 & 0.000 & \\
\hline 9th-11th grade & -236.519 & 546.839 & 0.672 & -448.776 & 810.522 & 0.589 & 737.665 & 602.165 & 0.242 \\
\hline High School Grad & 803.215 & 452.878 & 0.100 & 498.415 & 902.098 & 0.590 & 386.912 & 695.353 & 0.587 \\
\hline Some College/AA & 1427.999 & 451.493 & 0.007 & 520.372 & 883.760 & 0.566 & 503.276 & 619.118 & 0.431 \\
\hline College Grad or Above & 1434.140 & 420.501 & 0.005 & 1260.987 & 829.754 & 0.152 & 687.456 & 531.165 & 0.219 \\
\hline Multiple $\mathrm{R}^{2}$ & & 0.63 & & & 0.57 & & & 0.58 & \\
\hline
\end{tabular}

${ }^{a}$ Appendicular lean body mass ( $\mathrm{g}$ ) is an estimate from QDR 4500 fan beam DXA scan of lean muscle mass, excluding fat and bone; appendicular fat-free mass includes the arms, legs, hands and feet; some DXA values are multiply imputed due to nonrandom missing data

${ }^{\mathrm{b}}$ Analytic $\mathrm{n}$ shown; each person was weighted to account for complex survey design (including oversampling), survey non-response and post-stratification

${ }^{\mathrm{c}}$ Protein as $\%$ from animal is an average from two 24-hour recalls and = (Day 1 percent of protein intake from animal sources + Day 2 percent of protein intake from animal sources) / 2; animal protein included all meat, fish, poultry, eggs and dairy.

d Total energy intake (kcal) is an average from two 24-hour recalls

e Activity Level/Intensity is an average intensity value from physical activity accelerometer over a 4-7 day wear period; units correspond to physical activity METS

${ }^{\mathrm{f}}$ Age is reported in years at time of health screening; individuals $>85 \mathrm{y}$ are all topcoded at 85 years of age 


\subsection{Multiple Regression Analyses: Protein Intake as Predictive of Total Muscle Mass Index $\left(\mathrm{g} / \mathrm{m}^{2}\right)$}

Total Muscle Mass Index (MMI) was significantly, positively predicted by total grams of protein intake in men ages $19-50$ (p-value 0.023) and with protein as a percent of energy in women ages 19-50 (p-value 0.007). In other age-gender groups within these models or when protein was expressed as a percent contributed by animal foods, MMI was not significantly predicted by protein intake at alpha 0.05 (Tables 4.17-4.22).

While not significant at alpha 0.05 , total grams of protein intake moderately, positively predicted MMI in men ages 51-70 and women 19-50 (p-value $<0.10$, Tables 4.17 and 4.18). With the exception of women 19-50, as mentioned above, protein as a percent of energy was not predictive of MMI. Protein as a percent from animal foods was moderately, positively predictive of MMI in men ages $71+$ only but not in women (p-value $<0.01$, Tables 4.21 and 4.22).

Total energy intake was significantly negatively related to MMI for all expressions of protein intake in men $71+$ years and negatively related to MMI when protein was expressed as total grams of intake in men ages $51-70$ years ( $\mathrm{p}$-value $\leq 0.01$ ). Weight, used in place of BMI as a covariable to control for size, was significantly, positively related to MMI in men and women of all ages ( $\mathrm{p}$-value $<0.001)$. Physical activity level/intensity was not at all associated with MMI in women, but showed a significant, positive association in men ages $19-50$ and $71+$ years $(p$-value $<0.05)$ and a nearly significant, positive association in men 51-70 (p-value <0.06).

Age was not significantly associated with MMI in any age-gender group. Race/ethnicity was nearly always significantly associated with MMI, with Mexican 
Americans, other Hispanics, and non-Hispanic Blacks all generally demonstrating a greater MMI at a given protein intake when compared with non-Hispanic Whites. While rarely significant, a surprisingly negative overall trend was seen for the relationship between MMI and increasing levels of education in men and women of all ages. 
Table 4.17 Muscle Mass Index $\left(\mathrm{g} / \mathrm{m}^{2}\right)^{\mathrm{a}}$ as predicted by total daily protein intake $(\mathrm{g})$ in women

\begin{tabular}{|c|c|c|c|c|c|c|c|c|c|}
\hline & \multicolumn{3}{|c|}{ Women 19-50 $(n=578)^{b}$} & \multicolumn{3}{|c|}{ Women 51-70 $(n=474)^{b}$} & \multicolumn{3}{|c|}{ Women $71+(n=294)^{b}$} \\
\hline & $\beta$ & SE & $\mathrm{p}$ & $\beta$ & SE & $\mathrm{p}$ & $\beta$ & SE & $\mathrm{p}$ \\
\hline Total daily protein intake (g) & 5.036 & 2.700 & 0.092 & -4.370 & 5.003 & 0.399 & -0.183 & 4.624 & 0.969 \\
\hline Total Energy Intake (kcal) ${ }^{\mathrm{d}}$ & -0.184 & 0.155 & 0.259 & 0.025 & 0.208 & 0.904 & 0.050 & 0.310 & 0.874 \\
\hline Weight $(\mathrm{kg})$ & 111.005 & 3.911 & 0.000 & 124.415 & 5.051 & 0.000 & 116.157 & 5.103 & 0.000 \\
\hline Activity Level/Intensity ${ }^{\mathrm{e}}$ & 0.559 & 0.525 & 0.307 & 0.828 & 0.633 & 0.214 & 1.026 & 1.023 & 0.335 \\
\hline $\operatorname{Age}(y)^{\mathrm{f}}$ & 6.314 & 6.289 & 0.335 & -0.219 & 13.641 & 0.987 & 20.795 & 15.963 & 0.216 \\
\hline \multicolumn{10}{|l|}{ Race/Ethnicity } \\
\hline Non-Hispanic White (referent) & 0.000 & 0.000 & & 0.000 & 0.000 & & 0.000 & 0.000 & \\
\hline Mexican American & 569.749 & 180.272 & 0.008 & 696.303 & 239.178 & 0.012 & 576.608 & 432.746 & 0.206 \\
\hline Other Hispanic & 783.190 & 228.485 & 0.006 & 897.441 & 316.526 & 0.024 & -359.739 & 471.822 & 0.459 \\
\hline Non-Hispanic Black & 720.104 & 136.579 & 0.000 & 166.097 & 277.210 & 0.559 & 528.716 & 211.016 & 0.028 \\
\hline Other (includes multi-racial) & -196.489 & 266.738 & 0.474 & 649.556 & 295.410 & 0.049 & 1430.627 & 767.774 & 0.086 \\
\hline \multicolumn{10}{|l|}{ Education } \\
\hline Less than 9th grade (referent) & 0.000 & 0.000 & & 0.000 & 0.000 & & 0.000 & 0.000 & \\
\hline 9th-11th grade & -335.244 & 284.747 & 0.264 & -755.834 & 233.634 & 0.007 & -429.107 & 209.331 & 0.062 \\
\hline High School Grad & -635.406 & 260.830 & 0.034 & -406.499 & 312.326 & 0.215 & -85.685 & 205.268 & 0.683 \\
\hline Some College/AA & -601.263 & 260.846 & 0.040 & -695.703 & 416.546 & 0.119 & -569.759 & 229.538 & 0.031 \\
\hline College Grad or Above & -676.525 & 251.314 & 0.020 & -714.279 & 368.805 & 0.075 & -274.085 & 231.465 & 0.261 \\
\hline Multiple $\mathrm{R}^{2}$ & \multicolumn{3}{|c|}{0.81} & \multicolumn{3}{|c|}{0.73} & \multicolumn{3}{|c|}{0.73} \\
\hline
\end{tabular}

${ }^{a}$ Muscle Mass Index (MMI) is calculated from QDR 4500 fan beam DXA estimates of lean muscle mass, excluding fat and bone;

$\mathrm{MMI}=($ Fat-free mass $(\mathrm{g})) /\left(\right.$ Height $\left.\left(\mathrm{m}^{2}\right)\right)$; some DXA values are multiply imputed due to nonrandom missing data

${ }^{\mathrm{b}}$ Analytic $\mathrm{n}$ shown; each person was weighted to account for complex survey design (including oversampling), survey non-response and post-stratification

Total daily protein intake $(\mathrm{g})$ is an average from two $24 \mathrm{~h}$ recalls

${ }^{\mathrm{d}}$ Total energy intake ( $\mathrm{kcal}$ ) is an average from two $24 \mathrm{~h}$ recalls

${ }^{\mathrm{e}}$ Activity Level/Intensity is an average intensity value from physical activity accelerometer over a 4-7 day wear period; units correspond to physical activity METS

${ }^{\mathrm{f}}$ Age is reported in years at time of health screening; individuals $>85 \mathrm{y}$ are all topcoded at 85 years of age 
Table 4.18 Muscle Mass Index $\left(\mathrm{g} / \mathrm{m}^{2}\right)^{\mathrm{a}}$ as predicted by total daily protein intake $(\mathrm{g})$ in men

\begin{tabular}{|c|c|c|c|c|c|c|c|c|c|}
\hline & \multicolumn{3}{|c|}{ Men 19-50 $(n=648)^{b}$} & \multicolumn{3}{|c|}{ Men 51-70 $(n=435)^{b}$} & \multicolumn{3}{|c|}{ Men 71+ $(n=280)^{b}$} \\
\hline & $\beta$ & SE & $\mathrm{p}$ & $\beta$ & SE & $\mathrm{p}$ & $\beta$ & SE & $\mathrm{p}$ \\
\hline Total daily protein intake $(\mathrm{g})^{\mathrm{c}}$ & 4.903 & 1.902 & $\mathbf{0 . 0 2 3}$ & 7.007 & 3.857 & 0.092 & 4.800 & 4.292 & 0.284 \\
\hline Total Energy Intake $(\mathrm{kcal})^{\mathrm{d}}$ & -0.056 & 0.111 & 0.623 & -0.417 & 0.142 & 0.011 & -0.493 & 0.157 & 0.008 \\
\hline Weight $(\mathrm{kg})$ & 124.512 & 5.545 & 0.000 & 120.566 & 4.265 & 0.000 & 119.611 & 4.533 & 0.000 \\
\hline Activity Level/Intensity ${ }^{\mathrm{e}}$ & 1.078 & 0.388 & 0.016 & 1.614 & 0.762 & 0.054 & 1.789 & 0.754 & 0.039 \\
\hline $\operatorname{Age}(y)^{\mathrm{f}}$ & 1.661 & 7.477 & 0.828 & -5.694 & 16.411 & 0.734 & 17.052 & 19.021 & 0.388 \\
\hline \multicolumn{10}{|l|}{ Race/Ethnicity } \\
\hline Non-Hispanic White (referent) & 0.000 & 0.000 & & 0.000 & 0.000 & & 0.000 & 0.000 & \\
\hline Mexican American & 809.590 & 185.272 & 0.001 & 934.348 & 206.351 & 0.001 & 498.975 & 183.944 & 0.018 \\
\hline Other Hispanic & 1569.846 & 364.214 & 0.001 & 1118.894 & 205.886 & 0.000 & -736.031 & 245.187 & 0.010 \\
\hline Non-Hispanic Black & 748.155 & 141.077 & 0.000 & 835.899 & 252.539 & 0.006 & 315.826 & 246.920 & 0.225 \\
\hline Other (includes multi-racial) & 386.026 & 285.925 & 0.200 & 674.721 & 428.718 & 0.139 & 380.486 & 607.030 & 0.541 \\
\hline \multicolumn{10}{|l|}{ Education } \\
\hline Less than 9th grade (referent) & 0.000 & 0.000 & & 0.000 & 0.000 & & 0.000 & 0.000 & \\
\hline 9th-11th grade & -330.928 & 366.164 & 0.382 & 196.822 & 318.883 & 0.548 & -120.910 & 210.469 & 0.575 \\
\hline High School Grad & -504.063 & 328.837 & 0.149 & -282.391 & 293.052 & 0.353 & 260.930 & 273.003 & 0.357 \\
\hline Some College/AA & -143.644 & 301.065 & 0.641 & 87.305 & 312.395 & 0.784 & 70.706 & 246.167 & 0.778 \\
\hline College Grad or Above & -522.631 & 331.907 & 0.139 & -438.337 & 253.640 & 0.108 & -177.200 & 230.722 & 0.457 \\
\hline Multiple $\mathrm{R}^{2}$ & & 0.75 & & & 0.68 & & & 0.72 & \\
\hline
\end{tabular}

${ }^{a}$ Muscle Mass Index (MMI) is calculated from QDR 4500 fan beam DXA estimates of lean muscle mass, excluding fat and bone;

MMI $=($ Fat-free mass $(\mathrm{g})) /\left(\right.$ Height $\left.\left(\mathrm{m}^{2}\right)\right)$; some DXA values are multiply imputed due to nonrandom missing data

${ }^{\mathrm{b}}$ Analytic $\mathrm{n}$ shown; each person was weighted to account for complex survey design (including oversampling), survey non-response and post-stratification

${ }^{\mathrm{c}}$ Total daily protein intake $(\mathrm{g})$ is an average from two $24 \mathrm{~h}$ recalls

d Total energy intake (kcal) is an average from two $24 \mathrm{~h}$ recalls

${ }^{\mathrm{e}}$ Activity Level/Intensity is an average intensity value from physical activity accelerometer over a 4-7 day wear period; units correspond to physical activity METS

${ }^{\mathrm{f}}$ Age is reported in years at time of health screening; individuals $>85 \mathrm{y}$ are all topcoded at 85 years of age 
Table 4.19 Muscle Mass Index $\left(\mathrm{g} / \mathrm{m}^{2}\right)^{\mathrm{a}}$ as predicted by protein as a percent of total daily energy $(\%)$ in women

\begin{tabular}{|c|c|c|c|c|c|c|c|c|c|}
\hline & \multicolumn{3}{|c|}{ Women 19-50 $(n=578)^{b}$} & \multicolumn{3}{|c|}{ Women 51-70 $(n=474)^{b}$} & \multicolumn{3}{|c|}{ Women $71+(n=294)^{b}$} \\
\hline & $\beta$ & $\mathrm{SE}$ & $\mathrm{p}$ & $\beta$ & SE & $\mathrm{p}$ & $\beta$ & $\mathrm{SE}$ & $\mathrm{p}$ \\
\hline Protein as \% of energy & 34.480 & 10.509 & 0.007 & -19.008 & 20.192 & 0.364 & -2.365 & 19.545 & 0.906 \\
\hline Total Energy Intake (kcal) ${ }^{\mathrm{d}}$ & 0.030 & 0.096 & 0.758 & -0.147 & 0.089 & 0.122 & 0.042 & 0.187 & 0.828 \\
\hline Weight (kg) & 110.757 & 3.978 & 0.000 & 124.457 & 5.018 & 0.000 & 116.212 & 5.144 & 0.000 \\
\hline Activity Level/Intensity ${ }^{\mathrm{e}}$ & 0.542 & 0.531 & 0.326 & 0.813 & 0.626 & 0.218 & 1.022 & 1.023 & 0.337 \\
\hline $\operatorname{Age}(y)^{\mathrm{f}}$ & 7.215 & 6.264 & 0.271 & -0.645 & 13.791 & 0.963 & 20.766 & 15.953 & 0.216 \\
\hline \multicolumn{10}{|l|}{ Race/Ethnicity } \\
\hline Non-Hispanic White (referent) & 0.000 & 0.000 & & 0.000 & 0.000 & & 0.000 & 0.000 & \\
\hline Mexican American & 564.382 & 180.712 & 0.008 & 703.894 & 239.757 & 0.012 & 579.565 & 435.052 & 0.206 \\
\hline Other Hispanic & 763.204 & 229.014 & 0.007 & 889.345 & 315.749 & 0.025 & -357.620 & 470.070 & 0.460 \\
\hline Non-Hispanic Black & 717.937 & 133.851 & 0.000 & 167.084 & 278.572 & 0.559 & 528.853 & 212.681 & 0.029 \\
\hline Other (includes multi-racial) & -193.920 & 262.663 & 0.473 & 642.022 & 296.878 & 0.052 & 1431.711 & 766.768 & 0.085 \\
\hline \multicolumn{10}{|l|}{ Education } \\
\hline Less than 9th grade (referent) & 0.000 & 0.000 & & 0.000 & 0.000 & & 0.000 & 0.000 & \\
\hline 9th-11th grade & -334.917 & 298.834 & 0.286 & -757.754 & 229.856 & 0.006 & -428.159 & 209.432 & 0.063 \\
\hline High School Grad & -637.763 & 269.426 & 0.038 & -415.571 & 309.550 & 0.202 & -83.697 & 205.970 & 0.691 \\
\hline Some College/AA & -614.832 & 267.970 & 0.041 & -701.779 & 408.978 & 0.110 & -569.951 & 230.167 & 0.031 \\
\hline College Grad or Above & -682.723 & 263.499 & 0.024 & -721.415 & 356.016 & 0.064 & -270.803 & 231.808 & 0.267 \\
\hline Multiple $\mathrm{R}^{2}$ & & 0.81 & & & 0.73 & & & 0.73 & \\
\hline
\end{tabular}

${ }^{\mathrm{a}}$ Muscle Mass Index (MMI) is calculated from QDR 4500 fan beam DXA estimates of lean muscle mass, excluding fat and bone;

MMI $=($ Fat-free mass $(\mathrm{g})) /\left(\right.$ Height $\left.\left(\mathrm{m}^{2}\right)\right)$; some DXA values are multiply imputed due to nonrandom missing data

${ }^{\mathrm{b}}$ Analytic $\mathrm{n}$ shown; each person was weighted to account for complex survey design (including oversampling), survey non-response and post-stratification

${ }^{\mathrm{c}}$ Protein as $\%$ of energy is an average from two 24 -hour recalls and = (percent of energy from protein day $1+$ percent of energy from protein day 2 ) $/ 2$

d Total energy intake (kcal) is an average from two $24 \mathrm{~h}$ recalls

${ }^{\mathrm{e}}$ Activity Level/Intensity is an average intensity value from physical activity accelerometer over a 4-7 day wear period; units correspond to physical activity METS

${ }^{\mathrm{f}}$ Age is reported in years at time of health screening; individuals $>85 \mathrm{y}$ are all topcoded at 85 years of age 
Table 4.20 Muscle Mass Index $(\mathrm{g} / \mathrm{m} 2)^{\mathrm{a}}$ as predicted by protein as a percent of total daily energy $(\%)$ in men

\begin{tabular}{|c|c|c|c|c|c|c|c|c|c|}
\hline & \multicolumn{3}{|c|}{ Men 19-50 $(n=648)^{b}$} & \multicolumn{3}{|c|}{ Men 51-70 $(n=435)^{b}$} & \multicolumn{3}{|c|}{ Men 71+ $(n=280)^{b}$} \\
\hline & $\beta$ & SE & $\mathrm{p}$ & $\beta$ & SE & $\mathrm{p}$ & $\beta$ & SE & $\mathrm{p}$ \\
\hline Protein as \% of energy & 21.878 & 16.357 & 0.204 & 37.583 & 22.026 & 0.111 & 24.400 & 19.636 & 0.236 \\
\hline Total Energy Intake (kcal) ${ }^{\mathrm{d}}$ & 0.117 & 0.091 & 0.221 & -0.150 & 0.129 & 0.266 & -0.311 & 0.104 & 0.010 \\
\hline Weight $(\mathrm{kg})$ & 124.839 & 5.570 & 0.000 & 119.999 & 4.282 & 0.000 & 119.528 & 4.491 & 0.000 \\
\hline Activity Level/Intensity ${ }^{\mathrm{e}}$ & 1.092 & 0.393 & 0.016 & 1.602 & 0.753 & 0.053 & 1.831 & 0.745 & 0.034 \\
\hline $\operatorname{Age}(y)^{\mathrm{f}}$ & 1.779 & 7.609 & 0.819 & -5.350 & 16.242 & 0.747 & 17.211 & 18.562 & 0.372 \\
\hline \multicolumn{10}{|l|}{ Race/Ethnicity } \\
\hline Non-Hispanic White (referent) & 0.000 & 0.000 & & 0.000 & 0.000 & & 0.000 & 0.000 & \\
\hline Mexican American & 819.556 & 184.995 & 0.001 & 946.959 & 211.833 & 0.001 & 495.597 & 179.633 & 0.016 \\
\hline Other Hispanic & 1581.885 & 360.655 & 0.001 & 1052.108 & 241.562 & 0.001 & -736.834 & 247.301 & 0.011 \\
\hline Non-Hispanic Black & 741.484 & 143.468 & 0.000 & 837.795 & 247.336 & 0.005 & 322.849 & 244.981 & 0.212 \\
\hline Other (includes multi-racial) & 409.229 & 289.940 & 0.181 & 674.823 & 429.088 & 0.140 & 408.952 & 606.208 & 0.511 \\
\hline \multicolumn{10}{|l|}{ Education } \\
\hline Less than 9th grade (referent) & 0.000 & 0.000 & & 0.000 & 0.000 & & 0.000 & 0.000 & \\
\hline 9th-11th grade & -336.006 & 362.082 & 0.370 & 185.234 & 313.107 & 0.564 & -123.050 & 211.757 & 0.571 \\
\hline High School Grad & -501.703 & 330.867 & 0.153 & -297.700 & 284.304 & 0.314 & 261.670 & 273.189 & 0.356 \\
\hline Some College/AA & -149.688 & 302.915 & 0.629 & 85.703 & 310.961 & 0.787 & 67.322 & 244.283 & 0.787 \\
\hline College Grad or Above & -519.994 & 333.877 & 0.143 & -457.586 & 250.384 & 0.091 & -175.333 & 227.452 & 0.455 \\
\hline Multiple $\mathrm{R}^{2}$ & & 0.75 & & & 0.68 & & & 0.72 & \\
\hline
\end{tabular}

${ }^{\mathrm{a}}$ Muscle Mass Index (MMI) is calculated from QDR 4500 fan beam DXA estimates of lean muscle mass, excluding fat and bone;

MMI $=($ Fat-free mass $(\mathrm{g})) /\left(\right.$ Height $\left.\left(\mathrm{m}^{2}\right)\right)$; some DXA values are multiply imputed due to nonrandom missing data

${ }^{\mathrm{b}}$ Analytic $\mathrm{n}$ shown; each person was weighted to account for complex survey design (including oversampling), survey non-response and post-stratification

${ }^{\mathrm{c}}$ Protein as $\%$ of energy is an average from two 24 -hour recalls and $=$ (percent of energy from protein day $1+$ percent of energy from protein day 2 ) $/ 2$

${ }^{\mathrm{d}}$ Total energy intake ( $\mathrm{kcal}$ ) is an average from two $24 \mathrm{~h}$ recalls

${ }^{\mathrm{e}}$ Activity Level/Intensity is an average intensity value from physical activity accelerometer over a 4-7 day wear period; units correspond to physical activity METS

${ }^{\mathrm{f}}$ Age is reported in years at time of health screening; individuals $>85 \mathrm{y}$ are all topcoded at 85 years of age 
Table 4.21 Muscle Mass Index $\left(\mathrm{g} / \mathrm{m}^{2}\right)^{\mathrm{a}}$ as predicted by protein as a percent contributed by animal foods $(\%)$ in women

\begin{tabular}{|c|c|c|c|c|c|c|c|c|c|}
\hline & \multicolumn{3}{|c|}{ Women 19-50 $(n=578)^{b}$} & \multicolumn{3}{|c|}{ Women 51-70 $(n=474)^{b}$} & \multicolumn{3}{|c|}{ Women $71+(n=294)^{b}$} \\
\hline & $\beta$ & SE & $\mathrm{p}$ & $\beta$ & SE & $\mathrm{p}$ & $\beta$ & $\mathrm{SE}$ & $\mathrm{p}$ \\
\hline 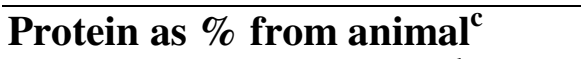 & -0.142 & 5.945 & 0.981 & -7.856 & 4.924 & 0.134 & -1.646 & 7.500 & 0.830 \\
\hline Total Energy Intake $(\mathrm{kcal})^{\mathrm{d}}$ & -0.037 & 0.105 & 0.731 & -0.114 & 0.095 & 0.252 & 0.042 & 0.192 & 0.830 \\
\hline Weight (kg) & 111.723 & 3.888 & 0.000 & 125.057 & 5.152 & 0.000 & 116.283 & 5.200 & 0.000 \\
\hline Activity Level/Intensity ${ }^{\mathrm{e}}$ & 0.535 & 0.496 & 0.301 & 0.759 & 0.599 & 0.228 & 1.005 & 1.058 & 0.360 \\
\hline $\operatorname{Age}(y)^{\mathrm{f}}$ & 5.566 & 6.289 & 0.393 & -2.354 & 13.778 & 0.867 & 20.774 & 16.023 & 0.218 \\
\hline \multicolumn{10}{|l|}{ Race/Ethnicity } \\
\hline Non-Hispanic White (referent) & 0.000 & 0.000 & & 0.000 & 0.000 & & 0.000 & 0.000 & \\
\hline Mexican American & 594.760 & 173.363 & 0.005 & 672.922 & 226.941 & 0.011 & 575.316 & 432.747 & 0.207 \\
\hline Other Hispanic & 812.534 & 233.849 & 0.005 & 918.745 & 324.255 & 0.023 & -365.242 & 479.233 & 0.459 \\
\hline Non-Hispanic Black & 713.801 & 131.473 & 0.000 & 187.360 & 278.056 & 0.512 & 529.616 & 213.511 & 0.029 \\
\hline Other (includes multi-racial) & -175.425 & 273.167 & 0.532 & 623.425 & 301.435 & 0.061 & 1432.057 & 777.908 & 0.089 \\
\hline \multicolumn{10}{|l|}{ Education } \\
\hline Less than 9th grade (referent) & 0.000 & 0.000 & & 0.000 & 0.000 & & 0.000 & 0.000 & \\
\hline 9th-11th grade & -292.285 & 263.404 & 0.292 & -734.061 & 224.305 & 0.006 & -427.753 & 211.602 & 0.065 \\
\hline High School Grad & -615.500 & 245.700 & 0.030 & -408.095 & 296.622 & 0.192 & -82.641 & 207.902 & 0.698 \\
\hline Some College/AA & -565.393 & 247.548 & 0.042 & -706.318 & 413.577 & 0.111 & -570.505 & 231.593 & 0.032 \\
\hline College Grad or Above & -625.750 & 253.175 & 0.030 & -749.941 & 360.269 & 0.058 & -271.891 & 244.061 & 0.288 \\
\hline Multiple $\mathrm{R}^{2}$ & & 0.81 & & & 0.73 & & & 0.73 & \\
\hline $\begin{array}{l}{ }^{\mathrm{a}} \text { Muscle Mass Index (MMI) is calculated } \\
\text { MMI= (Total body fat-free mass (g))/( } \\
\text { b Analytic n shown; each person was wei } \\
{ }^{\mathrm{c}} \text { Protein as \% from animal is an average } \\
\text { from animal sources) / } 2 \text {; animal protein } \\
\mathrm{d} \text { Total energy intake (kcal) is an average } \\
{ }^{\mathrm{e}} \text { Activity Level/Intensity is an average in } \\
\text { METS }\end{array}$ & $\begin{array}{l}\text { DR } 4500 \text { far } \\
\left.{ }^{2}\right) \text { ); some DX } \\
\text { account for } \\
\text { o 24-hour re } \\
\text { d all meat, fi } \\
\text { vo 24-hour re } \\
\text { value from p }\end{array}$ & $\begin{array}{l}\text { Deam DXA } \\
\text { A values are } \\
\text { mplex surv } \\
\text { lls and = (D } \\
\text { 1, poultry, e } \\
\text { alls } \\
\text { sical activit }\end{array}$ & $\begin{array}{l}\text { stimates } \\
\text { multiply i } \\
\text { y design } \\
\text { y } 1 \text { perce } \\
\text { gs and da }\end{array}$ & $\begin{array}{l}\text { f lean muscle } \\
\text { nputed due to } \\
\text { including ove } \\
\text { it of protein } \\
\text { ry. }\end{array}$ & $\begin{array}{l}\text { nass, exclud } \\
\text { honrandom } 1 \\
\text { sampling), s } \\
\text { take from an }\end{array}$ & $\begin{array}{l}\text { ig fat and } \\
\text { issing da } \\
\text { vey non- } \\
\text { mal sourc }\end{array}$ & $\begin{array}{l}\text { one; } \\
\text { sponse and } \mathrm{p} \\
\text { + Day } 2 \text { pei }\end{array}$ & $\begin{array}{l}\text { st-stratificat } \\
\text { ent of protei }\end{array}$ & intake \\
\hline
\end{tabular}


Table 4.22 Muscle Mass Index $\left(\mathrm{g} / \mathrm{m}^{2}\right)^{\mathrm{a}}$ as predicted by protein as a percent contributed by animal foods $(\%)$ in men

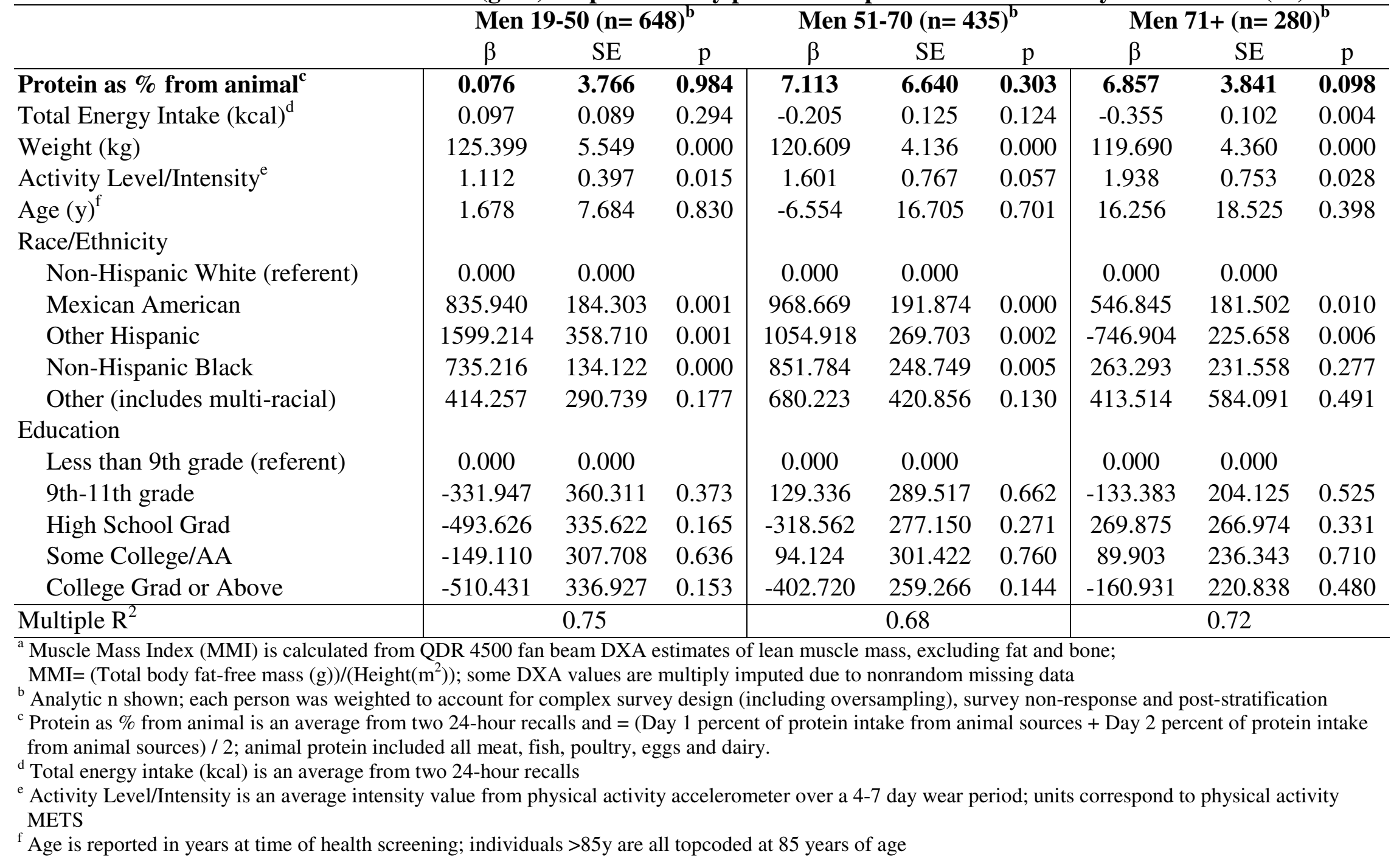




\subsection{Multiple Regression Analyses: Protein Intake as Predictive of Appendicular Muscle Mass Index $\left(\mathrm{g} / \mathrm{m}^{2}\right)$}

Appendicular Muscle Mass Index (AMMI) was significantly, positively predicted by total grams of protein intake in men ages $19-50$ and 51-70 (p-value $<0.05$, Table 4.24) but not in older men and not at all in women. Protein as a percent of energy was significantly, positively predictive of AMMI in women 19-50 years (p-value 0.024) and nearly positively significant in men 51-70 (p-value 0.053), as shown in tables 4.25 and 4.26. Protein as a percent contributed by animal foods was not significantly predictive of AMMI in any age-gender group (Tables 4.27 and 4.28).

Total energy intake was significantly, negatively related to AMMI in men 51-70 when protein was expressed as grams of intake and in men $71+$ when protein was expressed as grams of intake and as a percent contributed by animal foods ( $\mathrm{p}$-value $<0.05)$. Weight was significantly, positively related to AMMI in men and women of all ages (p-value <0.001). Physical activity level/intensity was not at all associated with AMMI in women, but showed a strong significant, positive association in men of all ages (p-value $<0.02$ )

Age was significantly, negatively associated with AMMI in men ages 19-50 for all expressions of protein intake but not in older men and not at all in women. Race/ethnicity was nearly always significantly associated with AMMI, with Mexican Americans, other Hispanics, and non-Hispanic Blacks again demonstrating a greater AMMI at a given protein intake when compared with non-Hispanic Whites. While very rarely significant, an overall negative trend was seen for the relationship between AMMI and increasing levels of education in men and women of all ages. 
Table 4.23 Appendicular Muscle Mass Index $\left(\mathrm{g} / \mathrm{m}^{2}\right)^{\mathrm{a}}$ as predicted by total daily protein intake (g) in women

\begin{tabular}{|c|c|c|c|c|c|c|c|c|c|}
\hline & \multicolumn{3}{|c|}{ Women 19-50 $(n=578)^{b}$} & \multicolumn{3}{|c|}{ Women 51-70 $(n=474)^{b}$} & \multicolumn{3}{|c|}{ Women 71+ $(n=294)^{b}$} \\
\hline & $\beta$ & SE & $\mathrm{p}$ & $\beta$ & SE & $\mathrm{p}$ & $\beta$ & $\mathrm{SE}$ & $\mathrm{p}$ \\
\hline Total daily protein intake $(\mathrm{g})^{\mathrm{c}}$ & 2.365 & 1.590 & 0.182 & -0.859 & 3.096 & 0.786 & 0.168 & 3.167 & $\mathbf{0 . 9 5 8}$ \\
\hline Total Energy Intake $(\mathrm{kcal})^{\mathrm{d}}$ & -0.092 & 0.079 & 0.269 & -0.009 & 0.113 & 0.936 & 0.011 & 0.197 & 0.957 \\
\hline Weight (kg) & 56.271 & 2.419 & 0.000 & 60.895 & 2.566 & 0.000 & 58.282 & 2.827 & 0.000 \\
\hline Activity Level/Intensity ${ }^{\mathrm{e}}$ & 0.585 & 0.284 & 0.060 & 0.693 & 0.400 & 0.107 & 0.814 & 0.640 & 0.228 \\
\hline $\operatorname{Age}(y)^{\mathrm{f}}$ & -4.696 & 3.553 & 0.211 & -0.226 & 6.436 & 0.973 & 14.969 & 8.345 & 0.097 \\
\hline \multicolumn{10}{|l|}{ Race/Ethnicity } \\
\hline Non-Hispanic White (referent) & 0.000 & 0.000 & & 0.000 & 0.000 & & 0.000 & 0.000 & \\
\hline Mexican American & 128.799 & 88.591 & 0.173 & 140.367 & 122.979 & 0.275 & 104.298 & 217.200 & 0.639 \\
\hline Other Hispanic & 378.880 & 141.347 & 0.022 & 486.699 & 208.642 & 0.050 & -239.235 & 286.775 & 0.419 \\
\hline Non-Hispanic Black & 743.527 & 88.908 & 0.000 & 508.912 & 153.049 & 0.005 & 715.106 & 132.771 & 0.000 \\
\hline Other (includes multi-racial) & -14.624 & 108.197 & 0.895 & 392.305 & 149.824 & 0.027 & 758.625 & 247.923 & 0.012 \\
\hline \multicolumn{10}{|l|}{ Education } \\
\hline Less than 9th grade (referent) & 0.000 & 0.000 & & 0.000 & 0.000 & & 0.000 & 0.000 & \\
\hline 9th-11th grade & -79.451 & 130.998 & 0.556 & -309.160 & 94.103 & 0.006 & -276.293 & 109.166 & 0.031 \\
\hline High School Grad & -274.409 & 115.312 & 0.040 & -18.047 & 162.577 & 0.913 & -31.264 & 98.990 & 0.758 \\
\hline Some College/AA & -152.006 & 98.385 & 0.155 & -84.612 & 173.689 & 0.634 & -312.969 & 132.857 & 0.038 \\
\hline College Grad or Above & -171.707 & 109.916 & 0.144 & -77.786 & 156.528 & 0.628 & -99.239 & 135.319 & 0.479 \\
\hline Multiple $\mathrm{R}^{2}$ & & 0.81 & & & 0.73 & & & 0.71 & \\
\hline
\end{tabular}

${ }^{a}$ Appendicular Muscle Mass Index (AMMI) is calculated from QDR 4500 fan beam DXA estimates of lean muscle mass, excluding fat and bone; AMMI= (Fat-

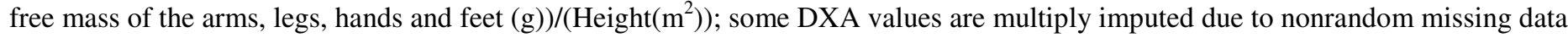

${ }^{\mathrm{b}}$ Analytic $\mathrm{n}$ shown; each person was weighted to account for complex survey design (including oversampling), survey non-response and post-stratification

${ }^{\mathrm{c}}$ Total daily protein intake $(\mathrm{g})$ is an average from two $24 \mathrm{~h}$ recalls

$\mathrm{d}$ Total energy intake $(\mathrm{kcal})$ is an average from two $24 \mathrm{~h}$ recalls

${ }^{\mathrm{e}}$ Activity Level/Intensity is an average intensity value from physical activity accelerometer over a 4-7 day wear period; units correspond to physical activity METS

${ }^{\mathrm{f}}$ Age is reported in years at time of health screening; individuals $>85 \mathrm{y}$ are all topcoded at 85 years of age 
Table 4.24 Appendicular Muscle Mass Index $\left(\mathrm{g} / \mathrm{m}^{2}\right)^{\mathrm{a}}$ as predicted by total protein intake $(\mathrm{g})$ in men

\begin{tabular}{|c|c|c|c|c|c|c|c|c|c|}
\hline & \multicolumn{3}{|c|}{ Men 19-50 $(n=648)^{b}$} & \multicolumn{3}{|c|}{ Men 51-70 $(n=435)^{b}$} & \multicolumn{3}{|c|}{ Men 71+ $(n=280)^{b}$} \\
\hline & $\beta$ & SE & $\mathrm{p}$ & $\beta$ & SE & $\mathrm{p}$ & $\beta$ & SE & $\mathrm{p}$ \\
\hline Total daily protein intake (g) & 2.846 & 1.253 & 0.041 & 5.180 & 2.234 & 0.038 & $\mathbf{1 . 7 7 0}$ & 2.018 & 0.396 \\
\hline Total Energy Intake $(\mathrm{kcal})^{\mathrm{d}}$ & -0.022 & 0.057 & 0.703 & -0.243 & 0.080 & 0.010 & -0.197 & 0.062 & 0.008 \\
\hline Weight $(\mathrm{kg})$ & 58.130 & 3.102 & 0.000 & 56.074 & 1.888 & 0.000 & 54.510 & 3.398 & 0.000 \\
\hline Activity Level/Intensity ${ }^{\mathrm{e}}$ & 0.698 & 0.221 & 0.008 & 1.307 & 0.449 & 0.012 & 1.452 & 0.384 & 0.003 \\
\hline $\operatorname{Age}(y)^{\mathrm{f}}$ & -9.482 & 3.314 & 0.014 & -14.773 & 8.197 & 0.095 & 8.580 & 10.777 & 0.441 \\
\hline \multicolumn{10}{|l|}{ Race/Ethnicity } \\
\hline Non-Hispanic White (referent) & 0.000 & 0.000 & & 0.000 & 0.000 & & 0.000 & 0.000 & \\
\hline Mexican American & 338.464 & 87.677 & 0.002 & 363.079 & 100.868 & 0.004 & 98.983 & 92.701 & 0.306 \\
\hline Other Hispanic & 690.934 & 151.464 & 0.001 & 532.765 & 106.022 & 0.000 & -225.789 & 198.748 & 0.276 \\
\hline Non-Hispanic Black & 943.651 & 87.221 & 0.000 & 927.171 & 110.251 & 0.000 & 594.411 & 125.679 & 0.000 \\
\hline Other (includes multi-racial) & 246.699 & 138.091 & 0.097 & 399.402 & 206.752 & 0.075 & -131.375 & 329.278 & 0.696 \\
\hline \multicolumn{10}{|l|}{ Education } \\
\hline Less than 9th grade (referent) & 0.000 & 0.000 & & 0.000 & 0.000 & & 0.000 & 0.000 & \\
\hline 9th-11th grade & -104.098 & 190.355 & 0.594 & 140.086 & 147.371 & 0.359 & -1.814 & 133.850 & 0.989 \\
\hline High School Grad & -173.082 & 177.705 & 0.348 & -2.104 & 130.079 & 0.987 & 120.555 & 129.049 & 0.368 \\
\hline Some College/AA & 94.165 & 164.644 & 0.577 & 119.580 & 145.749 & 0.427 & 8.620 & 110.927 & 0.939 \\
\hline College Grad or Above & -60.497 & 185.381 & 0.749 & 5.587 & 140.998 & 0.969 & -92.159 & 117.663 & 0.451 \\
\hline Multiple $\mathrm{R}^{2}$ & & 0.70 & & & 0.64 & & & 0.67 & \\
\hline
\end{tabular}

${ }^{a}$ Appendicular Muscle Mass Index (AMMI) is calculated from QDR 4500 fan beam DXA estimates of lean muscle mass, excluding fat and bone; AMMI= (Fatfree mass of the arms, legs, hands and feet $(\mathrm{g})) /\left(\operatorname{Height}\left(\mathrm{m}^{2}\right)\right)$; some DXA values are multiply imputed due to nonrandom missing data

${ }^{\mathrm{b}}$ Analytic $\mathrm{n}$ shown; each person was weighted to account for complex survey design (including oversampling), survey non-response and post-stratification

${ }^{\mathrm{c}}$ Total daily protein intake $(\mathrm{g})$ is an average from two $24 \mathrm{~h}$ recalls

d Total energy intake $(\mathrm{kcal})$ is an average from two $24 \mathrm{~h}$ recalls

${ }^{\mathrm{e}}$ Activity Level/Intensity is an average intensity value from physical activity accelerometer over a 4-7 day wear period; units correspond to physical activity METS

${ }^{\mathrm{f}}$ Age is reported in years at time of health screening; individuals $>85 \mathrm{y}$ are all topcoded at 85 years of age 
Table 4.25 Appendicular Muscle Mass Index $\left(\mathrm{g} / \mathrm{m}^{2}\right)^{\mathrm{a}}$ as predicted by protein as a percent of total daily energy $(\%)$ in women

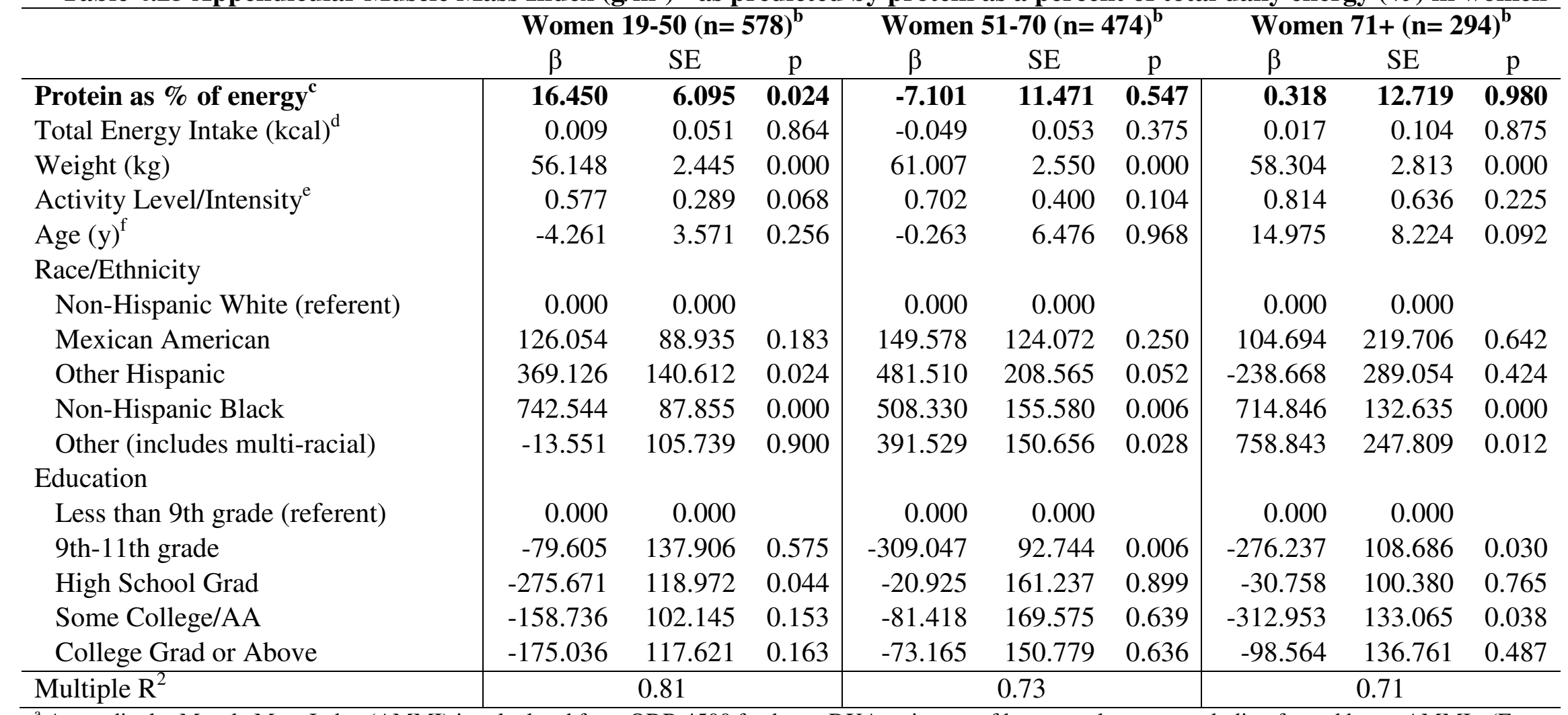

${ }^{a}$ Appendicular Muscle Mass Index (AMMI) is calculated from QDR 4500 fan beam DXA estimates of lean muscle mass, excluding fat and bone; AMMI= (Fatfree mass of the arms, legs, hands and feet $(\mathrm{g})) /\left(\operatorname{Height}\left(\mathrm{m}^{2}\right)\right)$; some DXA values are multiply imputed due to nonrandom missing data

${ }^{\mathrm{b}}$ Analytic n shown; each person was weighted to account for complex survey design (including oversampling), survey non-response and post-stratification

${ }^{\mathrm{c}}$ Protein as $\%$ of energy is an average from two 24 -hour recalls and $=$ (percent of energy from protein day $1+$ percent of energy from protein day 2 ) $/ 2$

${ }^{\mathrm{d}}$ Total energy intake (kcal) is an average from two $24 \mathrm{~h}$ recalls

${ }^{\mathrm{e}}$ Activity Level/Intensity is an average intensity value from physical activity accelerometer over a 4-7 day wear period; units correspond to physical activity METS

${ }^{\mathrm{f}}$ Age is reported in years at time of health screening; individuals $>85 \mathrm{y}$ are all topcoded at 85 years of age 
Table 4.26 Appendicular Muscle Mass Index $\left(\mathrm{g} / \mathrm{m}^{2}\right)^{\mathrm{a}}$ as predicted by protein as a percent of total daily energy $(\%)$ in men

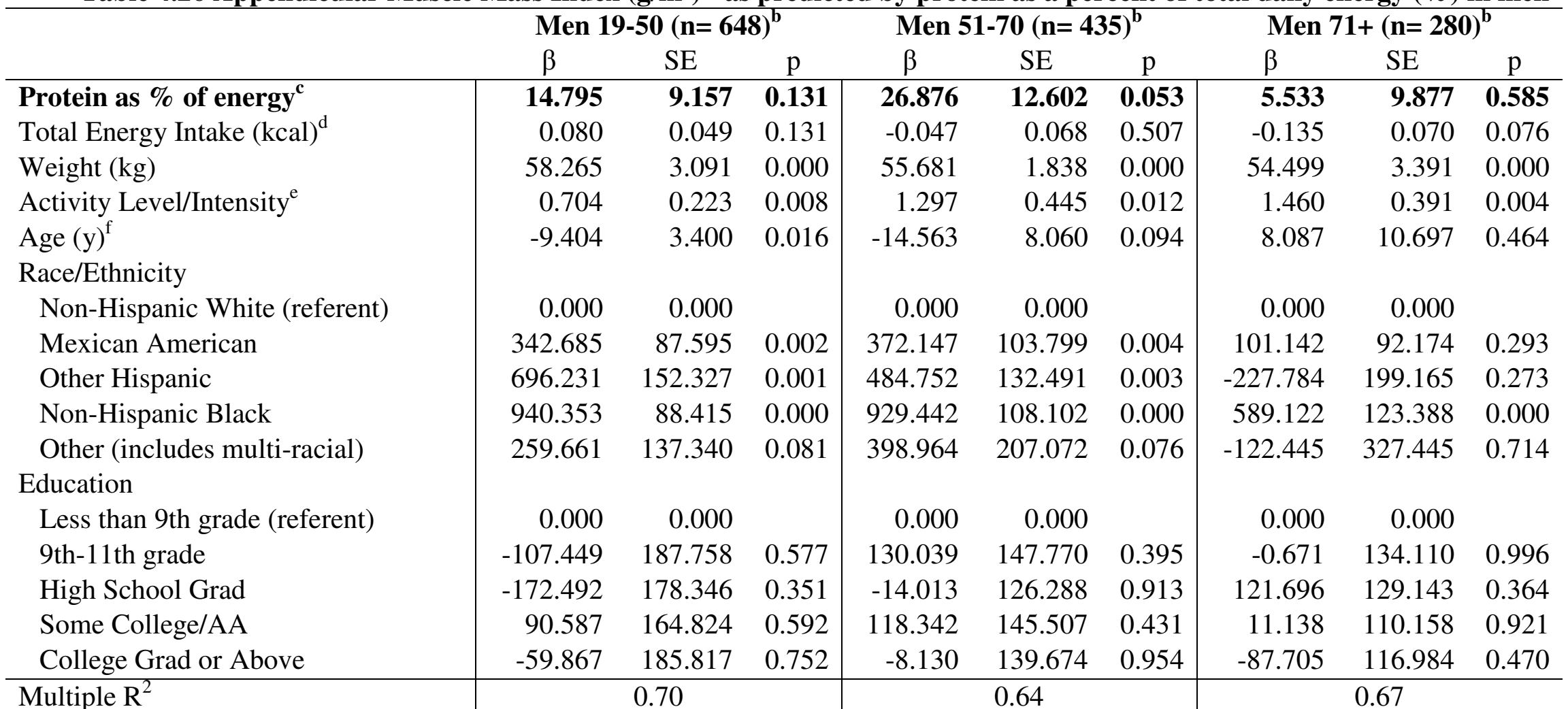

${ }^{\mathrm{a}}$ Appendicular Muscle Mass Index (AMMI) is calculated from QDR 4500 fan beam DXA estimates of lean muscle mass, excluding fat and bone; AMMI= (Fatfree mass of the arms, legs, hands and feet $(\mathrm{g})) /\left(\right.$ Height $\left.\left(\mathrm{m}^{2}\right)\right)$; some DXA values are multiply imputed due to nonrandom missing data

${ }^{\mathrm{b}}$ Analytic $\mathrm{n}$ shown; each person was weighted to account for complex survey design (including oversampling), survey non-response and post-stratification

${ }^{c}$ Protein as $\%$ of energy is an average from two 24 -hour recalls and $=$ (percent of energy from protein day $1+$ percent of energy from protein day 2 ) $/ 2$

${ }^{\mathrm{d}}$ Total energy intake (kcal) is an average from two $24 \mathrm{~h}$ recalls

${ }^{\mathrm{e}}$ Activity Level/Intensity is an average intensity value from physical activity accelerometer over a 4-7 day wear period; units correspond to physical activity METS

${ }^{\mathrm{f}}$ Age is reported in years at time of health screening; individuals $>85 \mathrm{y}$ are all topcoded at 85 years of age 
Table 4.27 Appendicular Muscle Mass Index $\left(\mathrm{g} / \mathrm{m}^{2}\right)^{\mathrm{a}}$ as predicted by protein as a percent contributed by animal foods $(\%)$ in women

\begin{tabular}{|c|c|c|c|c|c|c|c|c|c|}
\hline & \multicolumn{3}{|c|}{ Women 19-50 $(n=578)^{b}$} & \multicolumn{3}{|c|}{ Women 51-70 $(n=474)^{b}$} & \multicolumn{3}{|c|}{ Women $71+(n=294)^{b}$} \\
\hline & $\beta$ & SE & $\mathrm{p}$ & $\beta$ & SE & $\mathrm{p}$ & $\beta$ & SE & $\mathrm{p}$ \\
\hline Protein as \% from animal $^{\mathrm{c}}$ & 0.294 & 3.172 & 0.927 & -4.309 & 2.447 & 0.101 & -2.207 & 3.788 & 0.571 \\
\hline Total Energy Intake (kcal) ${ }^{\mathrm{d}}$ & -0.023 & 0.054 & 0.680 & -0.036 & 0.049 & 0.472 & 0.014 & 0.106 & 0.896 \\
\hline Weight (kg) & 56.593 & 2.423 & 0.000 & 61.437 & 2.612 & 0.000 & 58.533 & 2.705 & 0.000 \\
\hline Activity Level/Intensity ${ }^{\mathrm{e}}$ & 0.578 & 0.278 & 0.058 & 0.683 & 0.391 & 0.105 & 0.784 & 0.644 & 0.247 \\
\hline $\operatorname{Age}(y)^{\mathrm{f}}$ & -5.014 & 3.546 & 0.182 & -1.155 & 6.373 & 0.859 & 14.948 & 8.362 & 0.098 \\
\hline \multicolumn{10}{|l|}{ Race/Ethnicity } \\
\hline Non-Hispanic White (referent) & 0.000 & 0.000 & & 0.000 & 0.000 & & 0.000 & 0.000 & \\
\hline Mexican American & 139.807 & 84.547 & 0.125 & 140.220 & 115.376 & 0.246 & 104.847 & 212.704 & 0.630 \\
\hline Other Hispanic & 392.611 & 142.349 & 0.019 & 494.080 & 216.852 & 0.053 & -244.164 & 297.231 & 0.426 \\
\hline Non-Hispanic Black & 739.144 & 86.258 & 0.000 & 518.683 & 159.542 & 0.006 & 715.618 & 132.746 & 0.000 \\
\hline Other (includes multi-racial) & -6.089 & 111.270 & 0.957 & 382.027 & 154.788 & 0.034 & 761.585 & 255.771 & 0.013 \\
\hline \multicolumn{10}{|l|}{ Education } \\
\hline Less than 9th grade (referent) & 0.000 & 0.000 & & 0.000 & 0.000 & & 0.000 & 0.000 & \\
\hline 9th-11th grade & -62.757 & 121.261 & 0.615 & -295.566 & 88.609 & 0.006 & -273.949 & 112.037 & 0.035 \\
\hline High School Grad & -268.898 & 109.426 & 0.035 & -17.906 & 153.541 & 0.909 & -24.959 & 99.250 & 0.806 \\
\hline Some College/AA & -138.799 & 86.094 & 0.144 & -79.568 & 172.184 & 0.652 & -313.998 & 135.062 & 0.040 \\
\hline College Grad or Above & -149.912 & 108.399 & 0.192 & -82.854 & 149.485 & 0.589 & -93.087 & 146.696 & 0.539 \\
\hline Multiple $\mathrm{R}^{2}$ & & 0.81 & & & 0.73 & & & 0.71 & \\
\hline
\end{tabular}

${ }^{a}$ Appendicular Muscle Mass Index (AMMI) is calculated from QDR 4500 fan beam DXA estimates of lean muscle mass, excluding fat and bone; AMMI= (Fatfree mass of the arms, legs, hands and feet $(\mathrm{g})) /\left(\operatorname{Height}\left(\mathrm{m}^{2}\right)\right)$; some DXA values are multiply imputed due to nonrandom missing data

${ }^{\mathrm{b}}$ Analytic $\mathrm{n}$ shown; each person was weighted to account for complex survey design (including oversampling), survey non-response and post-stratification

${ }^{\mathrm{c}}$ Protein as $\%$ from animal is an average from two 24-hour recalls and = (Day 1 percent of protein intake from animal sources + Day 2 percent of protein intake from animal sources) / 2; animal protein included all meat, fish, poultry, eggs and dairy.

${ }^{\mathrm{d}}$ Total energy intake (kcal) is an average from two 24-hour recalls

${ }^{\mathrm{e}}$ Activity Level/Intensity is an average intensity value from physical activity accelerometer over a 4-7 day wear period; units correspond to physical activity METS

${ }^{\mathrm{f}}$ Age is reported in years at time of health screening; individuals $>85 \mathrm{y}$ are all topcoded at 85 years of age 
Table 4.28 Appendicular Muscle Mass Index $\left(\mathrm{g} / \mathrm{m}^{2}\right)^{\mathrm{a}}$ as predicted by protein as a percent contributed by animal foods $(\%)$ in men

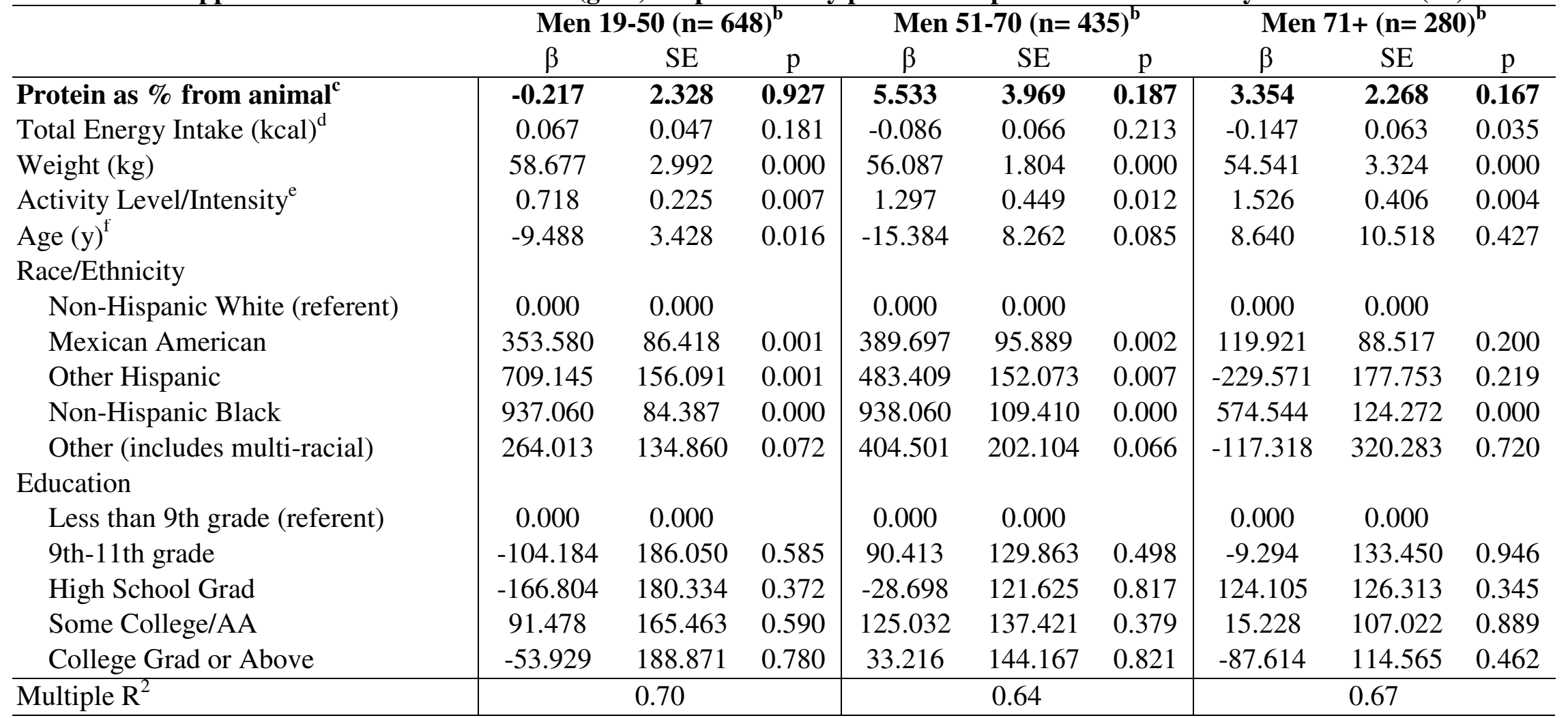

${ }^{a}$ Appendicular Muscle Mass Index (AMMI) is calculated from QDR 4500 fan beam DXA estimates of lean muscle mass, excluding fat and bone; AMMI= (Fatfree mass of the arms, legs, hands and feet $(\mathrm{g})) /\left(\operatorname{Height}\left(\mathrm{m}^{2}\right)\right)$; some DXA values are multiply imputed due to nonrandom missing data

${ }^{\mathrm{b}}$ Analytic $\mathrm{n}$ shown; each person was weighted to account for complex survey design (including oversampling), survey non-response and post-stratification

${ }^{\mathrm{c}}$ Protein as $\%$ from animal is an average from two 24-hour recalls and = (Day 1 percent of protein intake from animal sources + Day 2 percent of protein intake

from animal sources) / 2; animal protein included all meat, fish, poultry, eggs and dairy.

d Total energy intake (kcal) is an average from two 24-hour recalls

e Activity Level/Intensity is an average intensity value from physical activity accelerometer over a 4-7 day wear period; units correspond to physical activity METS

${ }^{\mathrm{f}}$ Age is reported in years at time of health screening; individuals $>85 \mathrm{y}$ are all topcoded at 85 years of age 


\subsection{Multiple Regression Analyses: Protein Intake as Predictive of Percent Lean Mass (\%)}

Percent lean mass was significantly, positively predicted by total grams of protein intake in men ages $71+(\mathrm{p}$-value 0.026$)$ and moderately, positively predictive in men 1950 (p-value 0.082) and women aged 71+ years (p-value 0.073), as shown in Tables 4.29 and 4.30. Protein as a percent of total energy was significantly, positively predictive of percent lean mass in men 71+ years (p-value 0.019$)$ and significantly, negatively predictive of percent lean mass in women 71+ years ( $\mathrm{p}$-value 0.046), as shown in Tables 4.31 and 4.32 . Protein as a percent contributed by animal foods was nearly positively predictive of percent lean mass in men 71+ (p-value 0.053) but not at all in women (Tables 4.33 and 4.34).

Total energy intake was significantly, positively related to percent lean mass in women 19-50 years when protein was expressed as a percent of total energy and in men 19-50 when protein was expressed as a percent of total energy or as a percent from animal foods $(\mathrm{p}<0.025)$. BMI group was nearly always significant as a strong predictor of percent lean mass, with underweight participants demonstrating a greater percent lean and overweight and obese participants demonstrating a lesser percent lean at a given protein intake when compared to the healthy referent group. Physical activity/intensity was positively, significantly related to percent lean mass in nearly all age-gender groups (p-value $<0.05)$.

Age was significantly, negatively associated with percent lean mass in men ages 5170 for all expressions of protein intake but not in other men and not at all in women. Race/ethnicity was not significantly associated with percent lean mass, with the exception 
of non-Hispanic Blacks who demonstrated a greater percent lean at a given protein intake when compared with non-Hispanic Whites. Education level was not significantly associated with percent lean in any age-gender group except for women 71+ years, where an overall negative trend was observed with increasing levels of education. 
Table 4.29 Percent lean mass $(\%)^{\text {a }}$ as predicted by total daily protein intake (g) in women

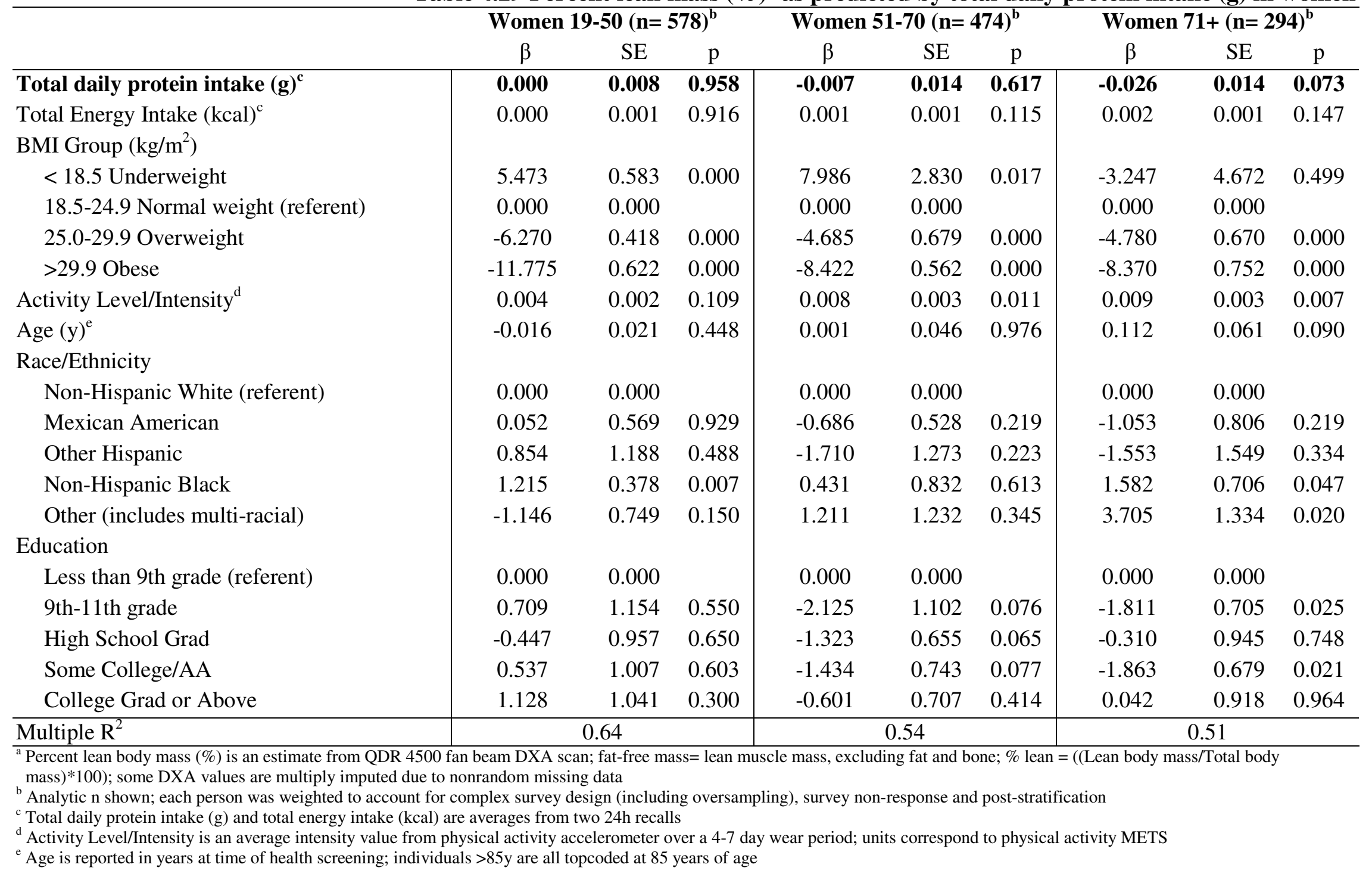


Table 4.30 Percent lean mass $(\%)^{\mathrm{a}}$ as predicted by total daily protein intake $(\mathrm{g})$ in men

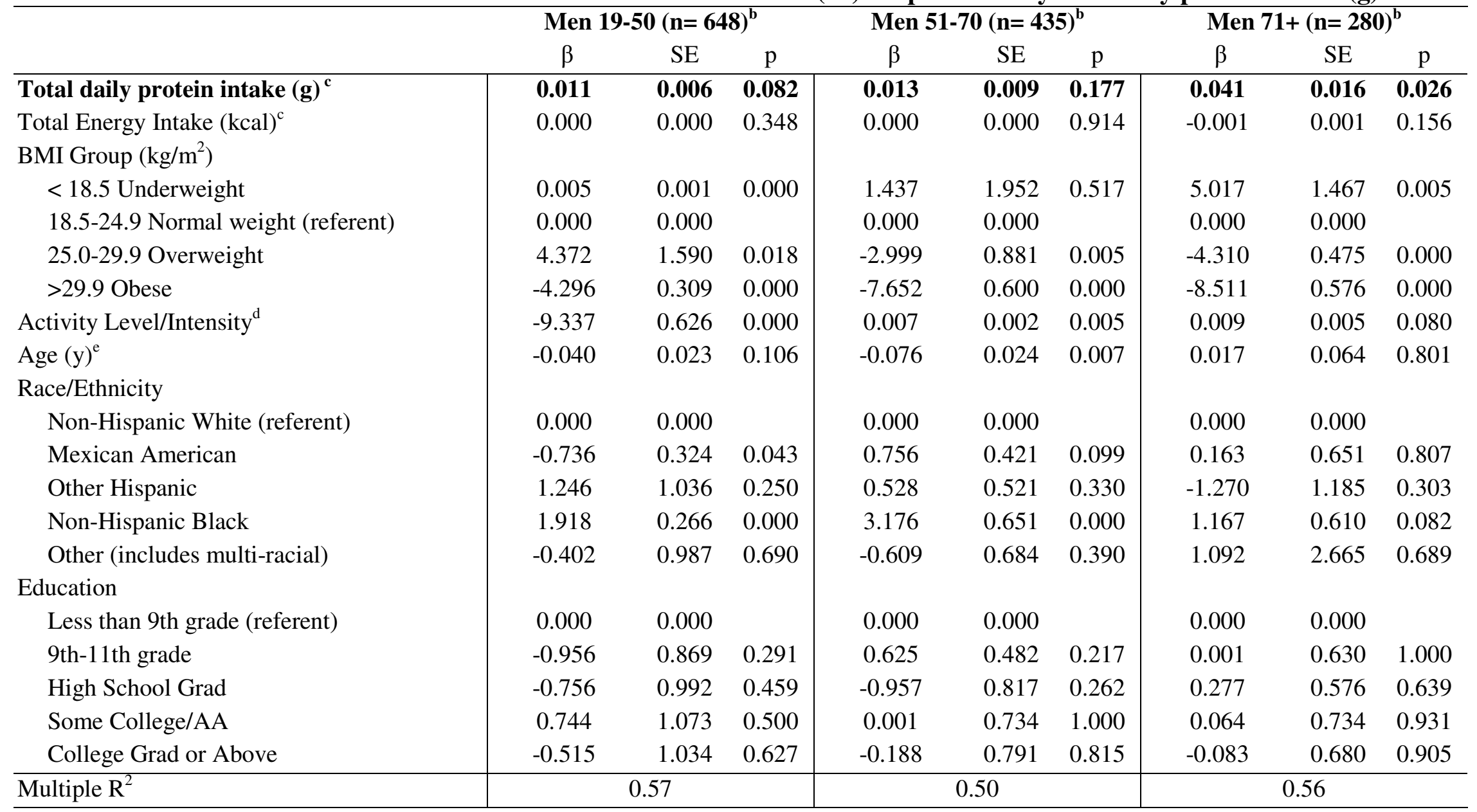

${ }^{a}$ Percent lean body mass (\%) is an estimate from QDR 4500 fan beam DXA scan; fat-free mass= lean muscle mass, excluding fat and bone; \% lean = ((Lean body mass/Total body mass)*100); some DXA values are multiply imputed due to nonrandom missing data

${ }^{b}$ Analytic $\mathrm{n}$ shown; each person was weighted to account for complex survey design (including oversampling), survey non-response and post-stratification

${ }^{\mathrm{c}}$ Total daily protein intake $(\mathrm{g})$ and total energy intake $(\mathrm{kcal})$ are averages from two $24 \mathrm{~h}$ recalls

d Activity Level/Intensity is an average intensity value from physical activity accelerometer over a 4-7 day wear period; units correspond to physical activity METS

${ }^{\mathrm{e}} \mathrm{Age}$ is reported in years at time of health screening; individuals $>85 \mathrm{y}$ are all topcoded at 85 years of age 
Table 4.31 Percent lean mass $(\%)^{\mathrm{a}}$ as predicted by protein as a percent of total daily energy $(\%)$ in women

\begin{tabular}{|c|c|c|c|c|c|c|c|c|c|}
\hline & \multicolumn{3}{|c|}{ Women 19-50 $(n=578)^{b}$} & \multicolumn{3}{|c|}{ Women 51-70 $(n=474)^{b}$} & \multicolumn{3}{|c|}{ Women $71+(n=294)^{b}$} \\
\hline & $\beta$ & SE & $\mathrm{p}$ & $\beta$ & SE & $\mathrm{p}$ & $\beta$ & SE & $\mathrm{p}$ \\
\hline Protein as \% of energy ${ }^{c}$ & $\mathbf{0 . 0 1 5}$ & $\mathbf{0 . 0 3 2}$ & 0.644 & -0.017 & 0.050 & 0.740 & -0.110 & $\mathbf{0 . 0 4 9}$ & $\overline{0.046}$ \\
\hline $\begin{array}{l}\text { Total Energy Intake }(\mathrm{kcal})^{\mathrm{d}} \\
\text { BMI Group }\left(\mathrm{kg} / \mathrm{m}^{2}\right)\end{array}$ & 5.492 & 0.574 & 0.000 & 0.001 & 0.000 & 0.124 & 0.001 & 0.001 & 0.439 \\
\hline$<18.5$ Underweight & -6.266 & 0.416 & 0.000 & 7.909 & 2.760 & 0.016 & -3.240 & 4.621 & 0.495 \\
\hline 18.5-24.9 Normal weight (referent) & 0.000 & 0.000 & & 0.000 & 0.000 & & 0.000 & 0.000 & \\
\hline 25.0-29.9 Overweight & -11.795 & 0.630 & 0.000 & -4.702 & 0.682 & 0.000 & -4.779 & 0.663 & 0.000 \\
\hline$>29.9$ Obese & 0.000 & 0.000 & 0.872 & -8.439 & 0.567 & 0.000 & -8.415 & 0.746 & 0.000 \\
\hline Activity Level/Intensity ${ }^{\mathrm{e}}$ & 0.004 & 0.002 & 0.110 & 0.008 & 0.003 & 0.011 & 0.009 & 0.003 & 0.008 \\
\hline $\operatorname{Age}(y)^{f}$ & -0.016 & 0.021 & 0.467 & 0.001 & 0.046 & 0.991 & 0.111 & 0.062 & 0.097 \\
\hline Race/Ethnicity & & & & & & & & & \\
\hline Non-Hispanic White (referent) & 0.000 & 0.000 & & 0.000 & 0.000 & & 0.000 & 0.000 & \\
\hline Mexican American & 0.039 & 0.574 & 0.947 & -0.705 & 0.534 & 0.212 & -1.011 & 0.803 & 0.234 \\
\hline Other Hispanic & 0.832 & 1.186 & 0.497 & -1.709 & 1.270 & 0.222 & -1.564 & 1.528 & 0.324 \\
\hline Non-Hispanic Black & 1.220 & 0.377 & 0.007 & 0.434 & 0.826 & 0.608 & 1.617 & 0.720 & 0.047 \\
\hline Other (includes multi-racial) & -1.154 & 0.741 & 0.144 & 1.201 & 1.230 & 0.348 & 3.717 & 1.357 & 0.021 \\
\hline Education & & & & & & & & & \\
\hline Less than 9th grade (referent) & 0.000 & 0.000 & & 0.000 & 0.000 & & 0.000 & 0.000 & \\
\hline 9th-11th grade & 0.687 & 1.176 & 0.570 & -2.133 & 1.098 & 0.074 & -1.790 & 0.689 & 0.024 \\
\hline High School Grad & -0.460 & 0.966 & 0.644 & -1.335 & 0.653 & 0.062 & -0.317 & 0.937 & 0.741 \\
\hline Some College/AA & 0.513 & 1.018 & 0.623 & -1.465 & 0.722 & 0.065 & -1.875 & 0.660 & 0.019 \\
\hline College Grad or Above & 1.096 & 1.063 & 0.323 & -0.639 & 0.691 & 0.375 & 0.049 & 0.914 & 0.958 \\
\hline Multiple $\mathrm{R}^{2}$ & & 0.65 & & & 0.54 & & & 0.50 & \\
\hline
\end{tabular}

${ }^{\mathrm{a}}$ Percent lean body mass (\%) is an estimate from QDR 4500 fan beam DXA scan; fat-free mass= lean muscle mass, excluding fat and bone; \% lean = ((Lean body mass/Total body mass)*100); some DXA values are multiply imputed due to nonrandom missing data

${ }^{\mathrm{b}}$ Analytic $\mathrm{n}$ shown; each person was weighted to account for complex survey design (including oversampling), survey non-response and post-stratification

${ }^{\mathrm{c}}$ Protein as $\%$ of energy is an average from two 24 -hour recalls and $=$ (percent of energy from protein day $1+$ percent of energy from protein day 2 ) $/ 2$

d Total energy intake (kcal) is an average from two 24-hour recalls

${ }^{\mathrm{e}}$ Activity Level/Intensity is an average intensity value from physical activity accelerometer over a 4-7 day wear period; units correspond to physical activity METS

${ }^{\mathrm{f}}$ Age is reported in years at time of health screening; individuals $>85 \mathrm{y}$ are all topcoded at 85 years of age 
Table 4.32 Percent lean mass $(\%)^{\mathrm{a}}$ as predicted by protein as a percent of total daily energy $(\%)$ in men

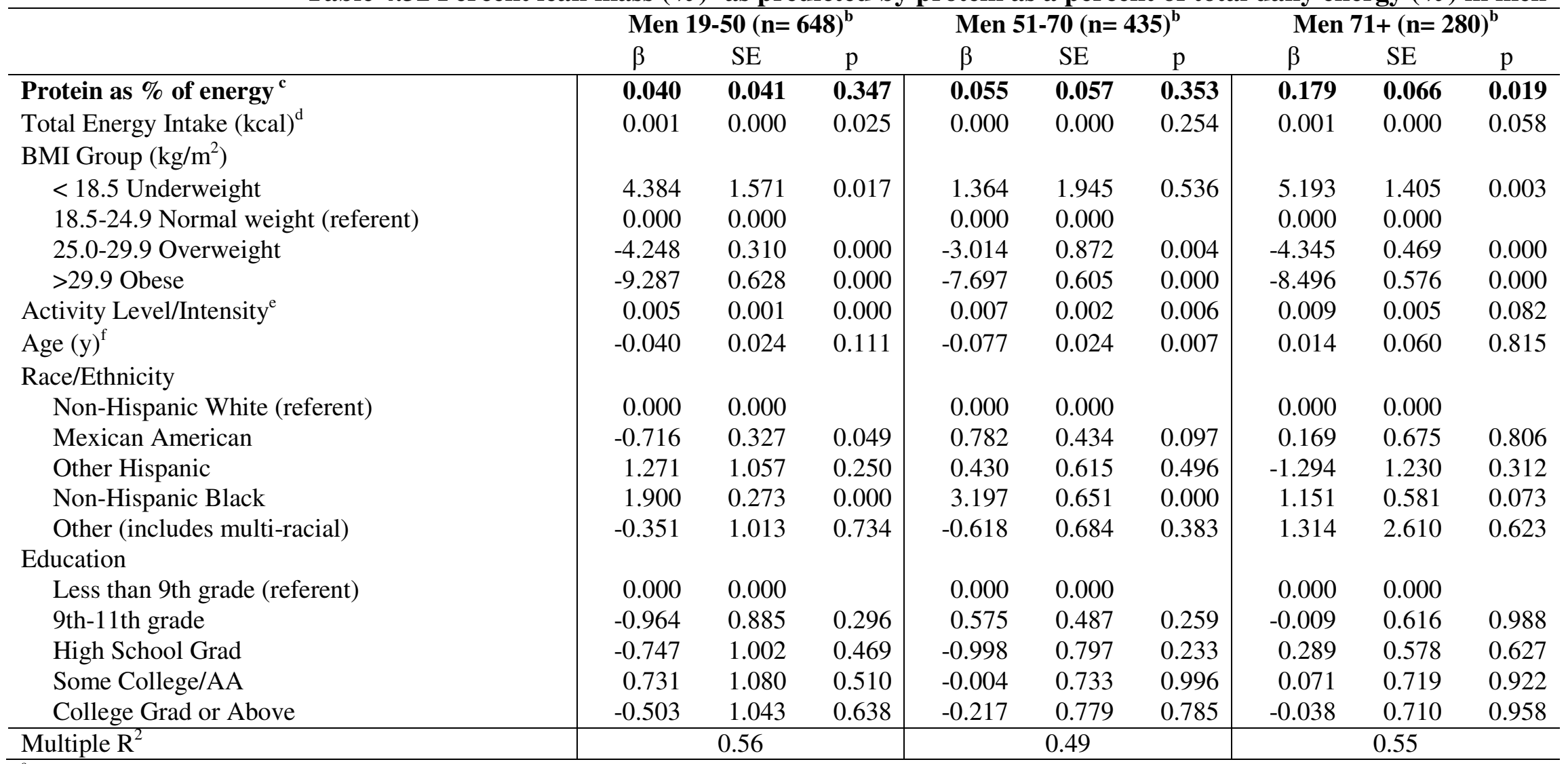

${ }^{\mathrm{a}}$ Percent lean body mass (\%) is an estimate from QDR 4500 fan beam DXA scan; fat-free mass= lean muscle mass, excluding fat and bone; \% lean = ((Lean body mass/Total body mass)*100); some DXA values are multiply imputed due to nonrandom missing data

${ }^{\mathrm{b}}$ Analytic $\mathrm{n}$ shown; each person was weighted to account for complex survey design (including oversampling), survey non-response and post-stratification

${ }^{c}$ Protein as $\%$ of energy is an average from two 24-hour recalls and = (percent of energy from protein day $1+$ percent of energy from protein day 2 ) $/ 2$

${ }^{\mathrm{d}}$ Total energy intake (kcal) is an average from two 24-hour recalls

${ }^{\mathrm{e}}$ Activity Level/Intensity is an average intensity value from physical activity accelerometer over a 4-7 day wear period; units correspond to physical activity METS

${ }^{\mathrm{f}}$ Age is reported in years at time of health screening; individuals $>85 \mathrm{y}$ are all topcoded at 85 years of age 
Table 4.33 Percent lean mass $(\%)^{\mathrm{a}}$ as predicted by protein as a percent contributed by animal foods (\%) in women

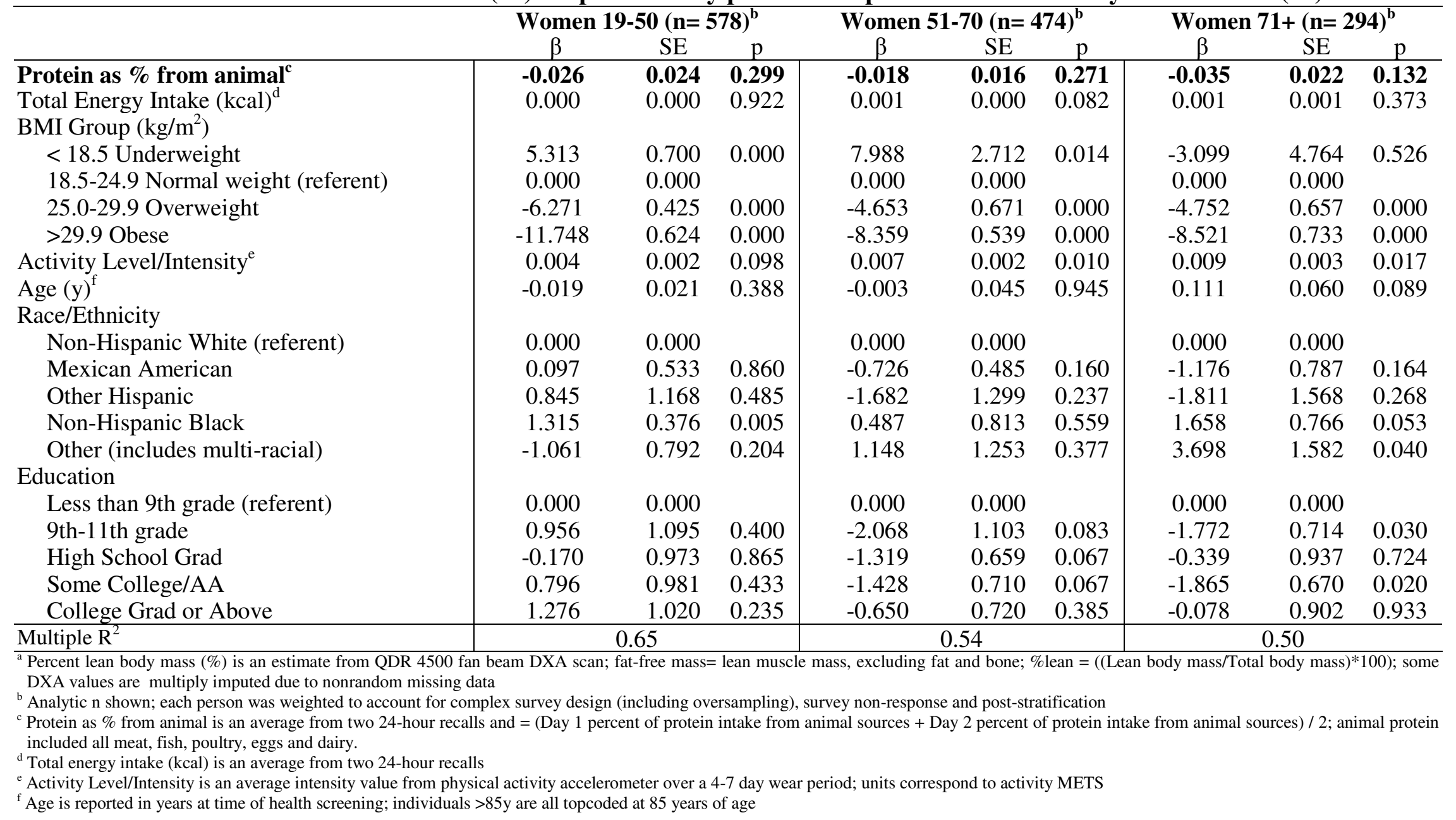


Table 4.34 Percent lean mass $(\%)^{\mathrm{a}}$ as predicted by protein as a percent contributed by animal foods (\%) in men

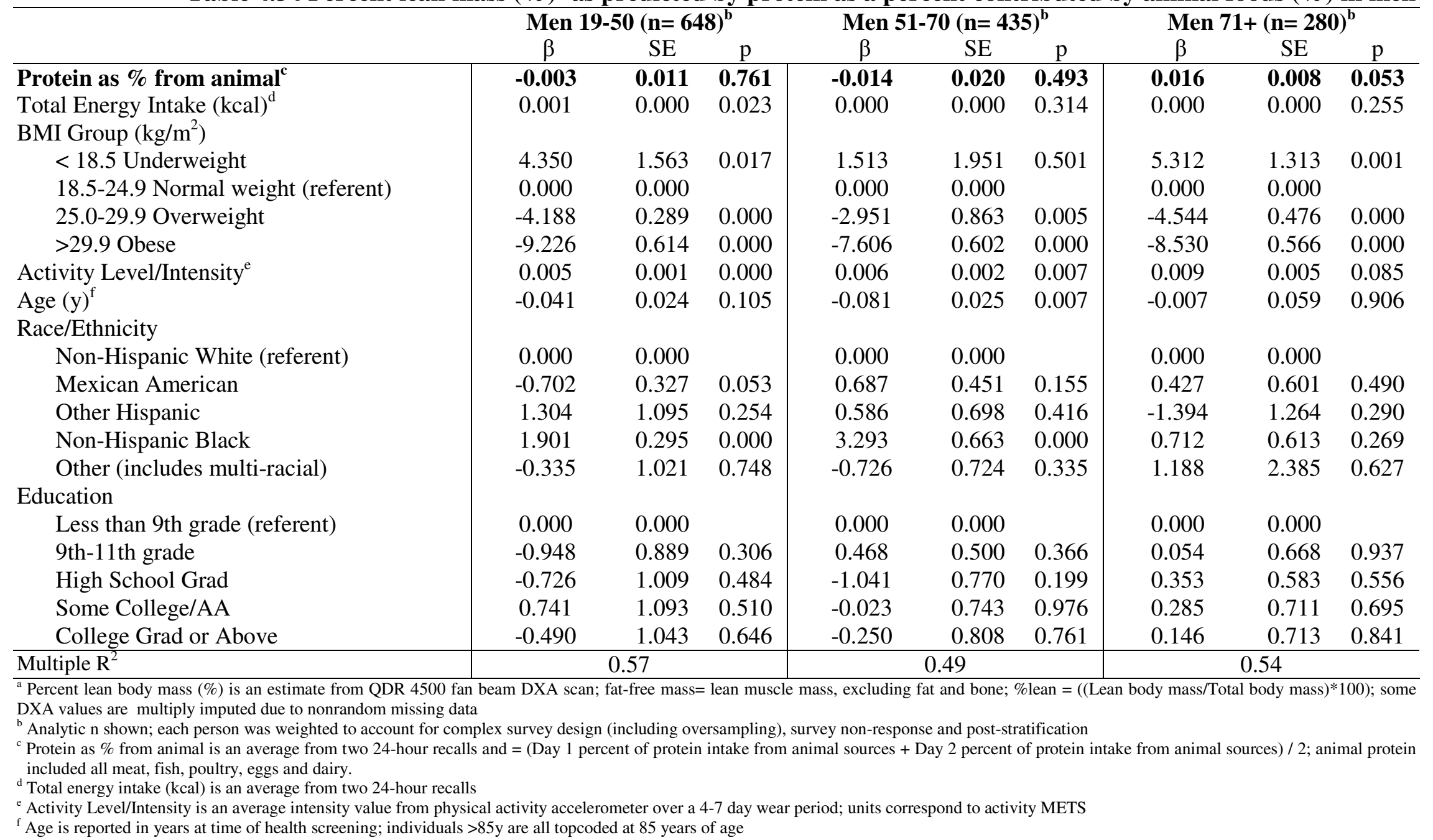




\subsection{Multiple Regression Analyses: Lean Body Mass as Predictive of Physical Limitation Score (PLS)}

When the measures of lean body mass, presented in Tables 4.3 and 4.4, were examined as predictive of physical functioning in adults ages 60 and over, results were surprising. Total and appendicular lean mass and total and appendicular muscle mass index were found to be significantly ( $\mathrm{p}$-value $<0.05$ ) or at least moderately ( $\mathrm{p}$-value $<0.10)$ positively predictive of physical limitation score (PLS) in nearly all age-gender groups, as shown in Tables 4.35-4.38. Since a higher PLS indicated a lesser functional ability, results showed that greater lean mass was associated with worse physical functioning. The only exception was percent lean mass (Table 4.39), which was significantly negatively predictive of PLS in men and women ages 60-70 but not at all significant in those aged $71+$.

Age was significantly, positively predictive of physical limitation in men and women $71+$ years only (p-value<0.01). BMI group, race/ethnicity and education level were not significantly predictive of physical limitation in any age-gender group.

General health status was nearly always predictive of physical limitation $(\mathrm{p}<0.05)$, with a higher PLS observed at poorer levels of health for a given lean mass, when compared to subjects reporting their health to be "excellent." Similarly, physical illness/injury status was significantly, positively related to PLS in all age-gender groups. 
Table 4.35 Physical Limitation Score (PLS) ${ }^{\mathrm{a}}$ as predicted by total lean mass $(\mathrm{kg})^{\mathrm{b}}$

\begin{tabular}{|c|c|c|c|c|c|c|c|c|c|c|c|c|}
\hline & \multicolumn{3}{|c|}{ Women 60-70 $(n=366)^{c}$} & \multicolumn{3}{|c|}{ Women $71+(n=388)^{c}$} & \multicolumn{3}{|c|}{ Men 60-70 $(n=333)^{c}$} & \multicolumn{3}{|c|}{ Men 71+ $(n=376)^{c}$} \\
\hline & $\beta$ & SE & $\mathrm{p}$ & $\beta$ & $\mathrm{SE}$ & $\mathrm{p}$ & $\beta$ & SE & $\mathrm{p}$ & $\beta$ & SE & $\mathrm{p}$ \\
\hline Total lean mass $(\mathrm{kg})^{\mathrm{b}}$ & 0.079 & 0.040 & 0.073 & 0.082 & 0.022 & 0.003 & 0.054 & 0.022 & 0.027 & 0.009 & 0.021 & 0.688 \\
\hline \multicolumn{13}{|l|}{ BMI Group $\left(\mathrm{kg} / \mathrm{m}^{2}\right)$} \\
\hline Underweight $<22.0$ & 0.657 & 0.406 & 0.129 & -0.172 & 0.252 & 0.506 & 0.621 & 0.399 & 0.144 & 0.257 & 0.583 & 0.667 \\
\hline Healthy Weight 22.0-27.0 (referent) & 0.000 & 0.000 & & 0.000 & 0.000 & & 0.000 & 0.000 & & 0.000 & 0.000 & \\
\hline Overweight $>27.0$ & -0.010 & 0.348 & 0.977 & 0.718 & 0.260 & 0.016 & -0.234 & 0.270 & 0.401 & 0.641 & 0.337 & 0.079 \\
\hline $\operatorname{Age}(y)^{d}$ & 0.003 & 0.030 & 0.930 & 0.121 & 0.021 & 0.000 & 0.005 & 0.031 & 0.874 & 0.134 & 0.042 & 0.007 \\
\hline \multicolumn{13}{|l|}{ Race/Ethnicity } \\
\hline Non-Hispanic White (referent) & 0.000 & 0.000 & & 0.000 & 0.000 & & 0.000 & 0.000 & & 0.000 & 0.000 & \\
\hline Mexican American & -0.140 & 0.374 & 0.713 & -0.679 & 0.514 & 0.209 & -0.232 & 0.281 & 0.424 & 0.251 & 0.450 & 0.586 \\
\hline Other Hispanic & -0.241 & 0.715 & 0.742 & -0.187 & 1.718 & 0.915 & -0.018 & 0.391 & 0.964 & 1.096 & 1.060 & 0.320 \\
\hline Non-Hispanic Black & 0.242 & 0.525 & 0.652 & -0.319 & 0.569 & 0.584 & -0.166 & 0.427 & 0.703 & -0.101 & 0.514 & 0.847 \\
\hline Other (Includes Multi-racial) & -0.021 & 0.474 & 0.966 & 0.141 & 0.471 & 0.770 & 0.440 & 0.700 & 0.540 & 0.814 & 0.897 & 0.380 \\
\hline \multicolumn{13}{|l|}{ Education } \\
\hline Less than 9th grade (referent) & 0.000 & 0.000 & & 0.000 & 0.000 & & 0.000 & 0.000 & & 0.000 & 0.000 & \\
\hline 9th-11th grade & 0.745 & 0.513 & 0.170 & -0.422 & 0.672 & 0.540 & -0.257 & 0.508 & 0.620 & 0.105 & 0.592 & 0.862 \\
\hline High School Grad & -0.171 & 0.330 & 0.614 & -1.018 & 0.613 & 0.120 & -0.078 & 0.546 & 0.888 & 0.274 & 0.466 & 0.566 \\
\hline Some College/AA & -0.084 & 0.321 & 0.797 & -0.723 & 0.607 & 0.254 & -0.320 & 0.451 & 0.490 & 0.126 & 0.395 & 0.754 \\
\hline College Grad or Above & -0.691 & 0.297 & 0.036 & -1.381 & 0.623 & 0.045 & -0.473 & 0.415 & 0.274 & 0.105 & 0.522 & 0.843 \\
\hline \multicolumn{13}{|l|}{ General Health Condition $^{\mathrm{e}}$} \\
\hline Excellent (referent) & 0.000 & 0.000 & & 0.000 & 0.000 & & 0.000 & 0.000 & & 0.000 & 0.000 & \\
\hline Very good & 0.518 & 0.208 & 0.027 & 0.887 & 0.431 & 0.059 & -0.042 & 0.145 & 0.774 & 0.094 & 0.368 & 0.802 \\
\hline Good & 0.755 & 0.333 & 0.041 & 1.047 & 0.307 & 0.005 & 0.870 & 0.285 & 0.009 & 0.211 & 0.437 & 0.637 \\
\hline Fair & 1.905 & 0.442 & 0.001 & 2.045 & 0.361 & 0.000 & 1.614 & 0.571 & 0.014 & 1.585 & 0.615 & 0.023 \\
\hline Poor & 2.731 & 0.583 & 0.000 & 3.653 & 0.589 & 0.000 & 3.018 & 1.140 & 0.020 & 2.009 & 0.802 & 0.026 \\
\hline Physical illness/injury status (d) ${ }^{\mathrm{f}}$ & 0.073 & 0.017 & 0.001 & 0.033 & 0.012 & 0.017 & 0.062 & 0.022 & 0.014 & 0.035 & 0.017 & 0.052 \\
\hline Multiple $\mathrm{R}^{2}$ & & 0.39 & & & 0.30 & & & 0.38 & & & 0.22 & \\
\hline
\end{tabular}


${ }^{\text {a }}$ Physical Limitation Score (PLS) = Index of disability in relation to performing specific functions of everyday living, unassisted: walking 1/4 mile, walking up 10 steps without resting, lifting or carrying 10lbs and standing from an armless straight chair. For each task a score of "0" was given for "no difficulty," "1" for "some difficulty," and "2" for "much difficulty," "unable to do," "do not do" or "missing." Scores for individual tasks were summed to total the PLS Score: 0-8 (Higher score indicates greater functional limitation)

$\mathrm{b}$ Total lean body mass (kg) is an estimate from QDR 4500 fan beam DXA scan; total lean body mass = lean muscle mass, excluding fat and bone; some DXA values are multiply imputed due to nonrandom missing data

${ }^{c}$ Analytic n shown; each person was weighted to account for complex survey design (including oversampling), survey non-response and post-stratification

${ }^{\mathrm{d}}$ Age is reported in years at time of health screening; individuals $>85 \mathrm{y}$ are all topcoded at 85 years of age

" General Health Condition from Current Health Status NHANES Questionnaire (HUQ.010) = "Would you say your general health is..."

${ }^{\mathrm{f}}$ Physical injury/illness status from Current Health Status NHANES Questionnaire (HSQ.470) = "Thinking about your physical health, which includes physical illness and injury, for how many days during the past 30 days was your physical health not good?" (Range $=0$-30 days) 
Table 4.36 Physical Limitation Score (PLS) ${ }^{\mathbf{a}}$ as predicted by appendicular lean mass (kg) ${ }^{\mathbf{b}}$

\begin{tabular}{|c|c|c|c|c|c|c|c|c|c|c|c|c|}
\hline & \multicolumn{3}{|c|}{ Women 60-70 $(n=366)^{c}$} & \multicolumn{3}{|c|}{ Women 71+ $(n=388)^{\mathrm{c}}$} & \multicolumn{3}{|c|}{ Men 60-70 $(n=333)^{c}$} & \multicolumn{3}{|c|}{ Men 71+ $(n=376)^{c}$} \\
\hline & $\beta$ & SE & $\mathrm{p}$ & $\beta$ & SE & $\mathrm{p}$ & $\beta$ & SE & $\mathrm{p}$ & $\beta$ & SE & $\mathrm{p}$ \\
\hline Appendicular lean mass $(\mathrm{kg})^{\mathbf{b}}$ & 0.145 & 0.082 & 0.101 & 0.168 & 0.041 & 0.001 & 0.084 & 0.044 & 0.078 & 0.023 & 0.046 & 0.622 \\
\hline \multicolumn{13}{|l|}{ BMI Group $\left(\mathrm{kg} / \mathrm{m}^{2}\right)$} \\
\hline Underweight $<22.0$ & 0.580 & 0.393 & 0.164 & -0.215 & 0.263 & 0.429 & 0.510 & 0.389 & 0.212 & 0.266 & 0.578 & 0.653 \\
\hline Healthy Weight 22.0-27.0 (referent) & 0.000 & 0.000 & & 0.000 & 0.000 & & 0.000 & 0.000 & & 0.000 & 0.000 & \\
\hline Overweight $>27.0$ & 0.031 & 0.340 & 0.930 & 0.733 & 0.260 & 0.014 & -0.068 & 0.232 & 0.774 & 0.627 & 0.331 & 0.080 \\
\hline Age $(y)^{d}$ & 0.004 & 0.028 & 0.895 & 0.119 & 0.021 & 0.000 & 0.002 & 0.031 & 0.943 & 0.135 & 0.042 & 0.007 \\
\hline \multicolumn{13}{|l|}{ Race/Ethnicity } \\
\hline Non-Hispanic White (referent) & 0.000 & 0.000 & & 0.000 & 0.000 & & 0.000 & 0.000 & & 0.000 & 0.000 & \\
\hline Mexican American & -0.168 & 0.389 & 0.673 & -0.655 & 0.510 & 0.222 & -0.305 & 0.286 & 0.306 & 0.262 & 0.441 & 0.562 \\
\hline Other Hispanic & -0.345 & 0.684 & 0.623 & -0.198 & 1.666 & 0.907 & -0.119 & 0.401 & 0.771 & 1.104 & 1.051 & 0.312 \\
\hline Non-Hispanic Black & 0.112 & 0.590 & 0.852 & -0.516 & 0.565 & 0.378 & -0.284 & 0.460 & 0.547 & -0.131 & 0.527 & 0.807 \\
\hline Other (Includes Multi-racial) & -0.054 & 0.472 & 0.911 & 0.081 & 0.496 & 0.873 & 0.363 & 0.720 & 0.622 & 0.832 & 0.904 & 0.374 \\
\hline \multicolumn{13}{|l|}{ Education } \\
\hline Less than 9th grade (referent) & 0.000 & 0.000 & & 0.000 & 0.000 & & 0.000 & 0.000 & & 0.000 & 0.000 & \\
\hline 9th-11th grade & 0.748 & 0.511 & 0.167 & -0.413 & 0.666 & 0.546 & -0.325 & 0.500 & 0.527 & 0.100 & 0.590 & 0.868 \\
\hline High School Grad & -0.189 & 0.328 & 0.574 & -1.026 & 0.599 & 0.110 & -0.117 & 0.530 & 0.829 & 0.271 & 0.464 & 0.568 \\
\hline Some College/AA & -0.114 & 0.331 & 0.735 & -0.693 & 0.600 & 0.268 & -0.311 & 0.418 & 0.470 & 0.128 & 0.401 & 0.754 \\
\hline College Grad or Above & -0.724 & 0.312 & 0.037 & -1.388 & 0.604 & 0.038 & -0.523 & 0.389 & 0.201 & 0.105 & 0.521 & 0.844 \\
\hline \multicolumn{13}{|l|}{ General Health Condition } \\
\hline Excellent (referent) & 0.000 & 0.000 & & 0.000 & 0.000 & & 0.000 & 0.000 & & 0.000 & 0.000 & \\
\hline Very good & 0.503 & 0.201 & 0.026 & 0.882 & 0.426 & 0.058 & -0.043 & 0.165 & 0.799 & 0.093 & 0.370 & 0.806 \\
\hline Good & 0.771 & 0.338 & 0.039 & 1.067 & 0.290 & 0.003 & 0.896 & 0.319 & 0.014 & 0.206 & 0.437 & 0.644 \\
\hline Fair & 1.945 & 0.446 & 0.001 & 2.090 & 0.352 & 0.000 & 1.654 & 0.608 & 0.017 & 1.593 & 0.602 & 0.020 \\
\hline Poor & 2.682 & 0.603 & 0.001 & 3.708 & 0.600 & 0.000 & 3.107 & 1.121 & 0.016 & 2.015 & 0.800 & 0.025 \\
\hline Physical illness/injury status $(\mathrm{d})^{\mathrm{f}}$ & 0.074 & 0.017 & 0.001 & 0.034 & 0.012 & 0.015 & 0.063 & 0.022 & 0.013 & 0.035 & 0.017 & 0.052 \\
\hline Multiple $\mathrm{R}^{2}$ & \multicolumn{3}{|c|}{0.38} & \multicolumn{3}{|c|}{0.31} & \multicolumn{3}{|c|}{0.36} & \multicolumn{3}{|c|}{0.22} \\
\hline
\end{tabular}


${ }^{\text {a }}$ Physical Limitation Score (PLS) = Index of disability in relation to performing specific functions of everyday living, unassisted: walking 1/4 mile, walking up 10 steps without resting, lifting or carrying 10lbs and standing from an armless straight chair. For each task a score of "0" was given for "no difficulty," "1" for "some difficulty," and "2" for "much difficulty," "unable to do," "do not do" or "missing." Scores for individual tasks were summed to total the PLS Score: 0-8 (Higher score indicates greater functional limitation)

${ }^{\mathrm{b}}$ Appendicular lean body mass (kg) is an estimate from QDR 4500 fan beam DXA scan; appendicular lean body mass = fat-free mass from the arms, legs, hands and feet, excluding fat and bone; some DXA values are multiply imputed due to nonrandom missing data

${ }^{\mathrm{c}}$ Analytic n shown; each person was weighted to account for complex survey design (including oversampling), survey non-response and post-stratification

${ }^{\mathrm{d}}$ Age is reported in years at time of health screening; individuals $>85 \mathrm{y}$ are all topcoded at 85 years of age

"General Health Condition from Current Health Status NHANES Questionnaire (HUQ.010) = "Would you say your general health is..."

${ }^{\mathrm{f}}$ Physical injury/illness status from Current Health Status NHANES Questionnaire (HSQ.470) = "Thinking about your physical health, which includes physical illness and injury, for how many days during the past 30 days was your physical health not good?" (Range $=0$-30 days) 
Table 4.37 Physical Limitation Score (PLS) as predicted by Muscle Mass Index $\left(\mathrm{kg} / \mathrm{m}^{2}\right)^{\mathrm{b}}$

\begin{tabular}{|c|c|c|c|c|c|c|c|c|c|c|c|c|}
\hline & \multicolumn{3}{|c|}{ Women 60-70 $(n=366)^{c}$} & \multicolumn{3}{|c|}{ Women $71+(n=388)^{c}$} & \multicolumn{3}{|c|}{ Men 60-70 $(n=333)^{c}$} & \multicolumn{3}{|c|}{ Men 71+ $(n=376)^{c}$} \\
\hline & $\beta$ & SE & $\mathrm{p}$ & $\beta$ & SE & $\mathrm{p}$ & $\beta$ & SE & $\mathrm{p}$ & $\beta$ & SE & $\mathrm{p}$ \\
\hline Muscle Mass Index $\left(\mathrm{kg} / \mathrm{m}^{2}\right)^{\mathrm{b}}$ & 0.226 & 0.097 & 0.037 & 0.300 & 0.066 & 0.001 & 0.246 & 0.077 & 0.007 & 0.119 & 0.117 & 0.330 \\
\hline \multicolumn{13}{|l|}{ BMI Group $\left(\mathrm{kg} / \mathrm{m}^{2}\right)$} \\
\hline Underweight $<22.0$ & 0.411 & 0.293 & 0.184 & -0.169 & 0.302 & 0.584 & 0.545 & 0.396 & 0.191 & 0.435 & 0.590 & 0.474 \\
\hline $\begin{array}{l}\text { Healthy Weight 22.0-27.0 } \\
\text { (referent) }\end{array}$ & 0.000 & 0.000 & & 0.000 & 0.000 & & 0.000 & 0.000 & & 0.000 & 0.000 & \\
\hline Overweight $>27.0$ & -0.076 & 0.311 & 0.811 & 0.465 & 0.282 & 0.125 & -0.444 & 0.231 & 0.077 & 0.377 & 0.489 & 0.454 \\
\hline $\operatorname{Age}(y)^{\mathrm{d}}$ & -0.020 & 0.027 & 0.476 & 0.108 & 0.021 & 0.000 & 0.010 & 0.030 & 0.747 & 0.139 & 0.043 & 0.007 \\
\hline \multicolumn{13}{|l|}{ Race/Ethnicity } \\
\hline Non-Hispanic White (referent) & 0.000 & 0.000 & & 0.000 & 0.000 & & 0.000 & 0.000 & & 0.000 & 0.000 & \\
\hline Mexican American & -0.111 & 0.416 & 0.793 & -0.910 & 0.458 & 0.068 & -0.379 & 0.256 & 0.162 & 0.268 & 0.440 & 0.552 \\
\hline Other Hispanic & -0.353 & 0.610 & 0.572 & -0.186 & 1.721 & 0.915 & -0.293 & 0.289 & 0.328 & 1.148 & 1.056 & 0.296 \\
\hline Non-Hispanic Black & 0.329 & 0.453 & 0.480 & -0.429 & 0.600 & 0.487 & -0.166 & 0.399 & 0.683 & -0.137 & 0.527 & 0.800 \\
\hline Other (Includes Multi-racial) & -0.128 & 0.483 & 0.795 & -0.114 & 0.528 & 0.832 & 0.347 & 0.692 & 0.624 & 0.851 & 0.919 & 0.371 \\
\hline \multicolumn{13}{|l|}{ Education } \\
\hline Less than 9th grade (referent) & 0.000 & 0.000 & & 0.000 & 0.000 & & 0.000 & 0.000 & & 0.000 & 0.000 & \\
\hline 9 th-11th grade & 1.192 & 0.571 & 0.057 & -0.419 & 0.709 & 0.564 & -0.133 & 0.390 & 0.738 & 0.158 & 0.596 & 0.794 \\
\hline High School Grad & 0.221 & 0.397 & 0.587 & -1.075 & 0.644 & 0.118 & 0.189 & 0.439 & 0.673 & 0.273 & 0.443 & 0.549 \\
\hline Some College/AA & 0.423 & 0.379 & 0.284 & -0.727 & 0.633 & 0.271 & -0.096 & 0.324 & 0.772 & 0.163 & 0.388 & 0.680 \\
\hline College Grad or Above & -0.206 & 0.371 & 0.589 & -1.373 & 0.653 & 0.055 & -0.099 & 0.326 & 0.766 & 0.190 & 0.523 & 0.722 \\
\hline \multicolumn{13}{|l|}{ General Health Condition $^{\mathrm{e}}$} \\
\hline Excellent (referent) & 0.000 & 0.000 & & 0.000 & 0.000 & & 0.000 & 0.000 & & 0.000 & 0.000 & \\
\hline Very good & 0.596 & 0.178 & 0.005 & 0.806 & 0.439 & 0.089 & -0.003 & 0.160 & 0.983 & 0.094 & 0.364 & 0.801 \\
\hline Good & 0.746 & 0.316 & 0.034 & 0.975 & 0.318 & 0.009 & 0.943 & 0.344 & 0.017 & 0.215 & 0.435 & 0.629 \\
\hline Fair & 1.765 & 0.392 & 0.001 & 1.912 & 0.390 & 0.000 & 1.651 & 0.570 & 0.012 & 1.591 & 0.605 & 0.020 \\
\hline Poor & 2.602 & 0.599 & 0.001 & 3.502 & 0.575 & 0.000 & 2.976 & 1.029 & 0.012 & 1.996 & 0.827 & 0.031 \\
\hline Physical illness/injury status $(\mathrm{d})^{\mathrm{f}}$ & 0.079 & 0.018 & 0.001 & 0.033 & 0.012 & 0.016 & 0.063 & 0.023 & 0.016 & 0.036 & 0.017 & 0.053 \\
\hline Multiple $\mathrm{R}^{2}$ & \multicolumn{3}{|c|}{0.39} & \multicolumn{3}{|c|}{0.31} & \multicolumn{3}{|c|}{0.39} & \multicolumn{3}{|c|}{0.23} \\
\hline
\end{tabular}


${ }^{\text {a }}$ Physical Limitation Score (PLS) = Index of disability in relation to performing specific functions of everyday living, unassisted: walking 1/4 mile, walking up 10 steps without resting, lifting or carrying $10 \mathrm{lbs}$ and standing from an armless straight chair. For each task a score of "0" was given for "no difficulty," " 1 " for "some difficulty," and "2" for much difficulty," "unable to do," "do not do" or "missing." Scores for individual tasks were summed to total the PLS Score: 0-8 (Higher score indicates greater functional limitation)

${ }^{\mathrm{b}}$ Muscle Mass Index $\left(\mathrm{kg} / \mathrm{m}^{2}\right)$ is an estimate from QDR 4500 fan beam DXA scan of lean muscle mass, excluding fat and bone; Muscle Mass Index $(\mathrm{MMI})=(($ Fat-free mass $(\mathrm{kg}) /$ Height $(\mathrm{m} 2))$; some DXA values are multiply imputed due to nonrandom missing data

${ }^{\mathrm{c}}$ Analytic $\mathrm{n}$ shown; each person was weighted to account for complex survey design (including oversampling), survey non-response and post-stratification

${ }^{\mathrm{d}}$ Age is reported in years at time of health screening; individuals $>85 \mathrm{y}$ are all topcoded at 85 years of age

e General Health Condition from Current Health Status NHANES Questionnaire (HUQ.010) = "Would you say your general health is..."

${ }^{\mathrm{f}}$ Physical injury/illness status from Current Health Status NHANES Questionnaire (HSQ.470) = "Thinking about your physical health, which includes physical illness and injury, for how many days during the past 30 days was your physical health not good?" (Range $=0-30$ days) 
Table 4.38 Physical Limitation Score (PLS) as predicted by Appendicular Muscle Mass Index $\left(\mathrm{kg} / \mathrm{m}^{2}\right)^{\mathrm{b}}$

\begin{tabular}{|c|c|c|c|c|c|c|c|c|c|c|c|c|}
\hline & \multicolumn{3}{|c|}{ Women 60-70 $(n=366)^{c}$} & \multicolumn{3}{|c|}{ Women $71+(n=388)^{c}$} & \multicolumn{3}{|c|}{ Men 60-70 $(n=333)^{c}$} & \multicolumn{3}{|c|}{ Men $71+(n=376)^{c}$} \\
\hline & $\beta$ & SE & $\mathrm{p}$ & $\beta$ & SE & $\mathrm{p}$ & $\beta$ & SE & $\mathrm{p}$ & $\beta$ & SE & $\mathrm{p}$ \\
\hline $\begin{array}{l}\text { Appendicular Muscle Mass Index } \\
\left(\mathbf{k g} / \mathbf{m}^{\mathbf{2}}\right)^{\mathbf{b}} \\
\text { BMI Group }\left(\mathrm{kg} / \mathrm{m}^{2}\right)\end{array}$ & 0.407 & 0.215 & 0.081 & 0.560 & 0.125 & 0.001 & 0.354 & 0.161 & 0.047 & 0.208 & 0.238 & 0.398 \\
\hline Underweight $<22.0$ & 0.316 & 0.290 & 0.295 & -0.235 & 0.320 & 0.476 & 0.366 & 0.406 & 0.383 & 0.372 & 0.574 & 0.527 \\
\hline Healthy Weight 22.0-27.0 (referent) & 0.000 & 0.000 & & 0.000 & 0.000 & & 0.000 & 0.000 & & 0.000 & 0.000 & \\
\hline Overweight $>27.0$ & -0.009 & 0.319 & 0.979 & 0.560 & 0.276 & 0.063 & -0.167 & 0.208 & 0.438 & 0.454 & 0.445 & 0.326 \\
\hline $\operatorname{Age}(y)^{\mathrm{d}}$ & -0.015 & 0.026 & 0.583 & 0.107 & 0.021 & 0.000 & 0.007 & 0.030 & 0.810 & 0.139 & 0.044 & 0.007 \\
\hline \multicolumn{13}{|l|}{ Race/Ethnicity } \\
\hline Non-Hispanic White (referent) & 0.000 & 0.000 & & 0.000 & 0.000 & & 0.000 & 0.000 & & 0.000 & 0.000 & \\
\hline Mexican American & -0.094 & 0.408 & 0.822 & -0.825 & 0.463 & 0.098 & -0.388 & 0.264 & 0.166 & 0.285 & 0.432 & 0.521 \\
\hline Other Hispanic & -0.408 & 0.585 & 0.498 & -0.200 & 1.653 & 0.906 & -0.278 & 0.316 & 0.393 & 1.140 & 1.068 & 0.305 \\
\hline Non-Hispanic Black & 0.189 & 0.513 & 0.718 & -0.659 & 0.601 & 0.293 & -0.323 & 0.443 & 0.479 & -0.212 & 0.569 & 0.716 \\
\hline Other (Includes Multi-racial) & -0.119 & 0.471 & 0.804 & -0.138 & 0.543 & 0.803 & 0.306 & 0.718 & 0.677 & 0.875 & 0.913 & 0.355 \\
\hline \multicolumn{13}{|l|}{ Education } \\
\hline Less than 9th grade (referent) & 0.000 & 0.000 & & 0.000 & 0.000 & & 0.000 & 0.000 & & 0.000 & 0.000 & \\
\hline 9th-11th grade & 1.158 & 0.562 & 0.059 & -0.419 & 0.701 & 0.560 & -0.215 & 0.396 & 0.596 & 0.141 & 0.588 & 0.814 \\
\hline High School Grad & 0.173 & 0.364 & 0.643 & -1.101 & 0.627 & 0.102 & 0.092 & 0.432 & 0.835 & 0.281 & 0.444 & 0.537 \\
\hline Some College/AA & 0.333 & 0.353 & 0.362 & -0.706 & 0.626 & 0.279 & -0.120 & 0.306 & 0.701 & 0.179 & 0.402 & 0.664 \\
\hline College Grad or Above & -0.293 & 0.342 & 0.406 & -1.404 & 0.629 & 0.043 & -0.248 & 0.311 & 0.439 & 0.185 & 0.520 & 0.727 \\
\hline \multicolumn{13}{|l|}{ General Health Condition $^{\mathrm{e}}$} \\
\hline Excellent (referent) & 0.000 & 0.000 & & 0.000 & 0.000 & & 0.000 & 0.000 & & 0.000 & 0.000 & \\
\hline Very good & 0.560 & 0.176 & 0.007 & 0.819 & 0.429 & 0.078 & -0.022 & 0.178 & 0.905 & 0.104 & 0.365 & 0.780 \\
\hline Good & 0.760 & 0.321 & 0.033 & 1.014 & 0.294 & 0.004 & 0.958 & 0.372 & 0.023 & 0.204 & 0.433 & 0.646 \\
\hline Fair & 1.809 & 0.390 & 0.000 & 1.988 & 0.374 & 0.000 & 1.706 & 0.619 & 0.016 & 1.615 & 0.592 & 0.017 \\
\hline Poor & 2.570 & 0.619 & 0.001 & 3.601 & 0.589 & 0.000 & 3.133 & 1.035 & 0.009 & 2.013 & 0.825 & 0.029 \\
\hline Physical illness/injury status $(\mathrm{d})^{\mathrm{f}}$ & 0.080 & 0.018 & 0.001 & 0.034 & 0.012 & 0.015 & 0.064 & 0.022 & 0.014 & 0.036 & 0.017 & 0.055 \\
\hline Multiple $\mathrm{R}^{2}$ & \multicolumn{3}{|c|}{0.39} & \multicolumn{3}{|c|}{0.31} & \multicolumn{3}{|c|}{0.37} & \multicolumn{3}{|c|}{0.23} \\
\hline
\end{tabular}


${ }^{\text {a }}$ Physical Limitation Score (PLS) = Index of disability in relation to performing specific functions of everyday living, unassisted: walking 1/4 mile, walking up 10 steps without resting, lifting or carrying 10lbs and standing from an armless straight chair. For each task a score of "0" was given for "no difficulty," "1" for "some difficulty," and "2" for much difficulty," "unable to do," "do not do" or "missing." Scores for individual tasks were summed to total the PLS Score: 0-8 (Higher score indicates greater functional limitation)

${ }^{\mathrm{b}}$ Appendicular Muscle Mass Index $\left(\mathrm{kg} / \mathrm{m}^{2}\right)$ is an estimate from QDR 4500 fan beam DXA scan of lean muscle mass, excluding fat and bone; Appendicular Muscle Mass Index

$(\mathrm{AMMI})=(($ Fat-free mass of the arms, legs, hands and feet $(\mathrm{kg}) /$ Height $(\mathrm{m} 2))$;some DXA values are multiply imputed due to nonrandom missing data ${ }^{c}$ Analytic $\mathrm{n}$ shown; each person was weighted to account for complex survey design (including oversampling), survey non-response and post-stratification

d Age is reported in years at time of health screening; individuals $>85 \mathrm{y}$ are all topcoded at 85 years of age

" General Health Condition from Current Health Status NHANES Questionnaire (HUQ.010) = "Would you say your general health is..."

${ }^{\mathrm{f}}$ Physical injury/illness status from Current Health Status NHANES Questionnaire (HSQ.470) = "Thinking about your physical health, which includes physical illness and injury, for how many days during the past 30 days was your physical health not good?" (Range $=0-30$ days) 
Table 4.39 Physical Limitation Score (PLS) as predicted by percent lean mass $(\%)^{\mathrm{b}}$

\begin{tabular}{|c|c|c|c|c|c|c|c|c|c|c|c|c|}
\hline & \multicolumn{3}{|c|}{ Women 60-70 $(n=366)^{c}$} & \multicolumn{3}{|c|}{ Women $71+(n=388)^{c}$} & \multicolumn{3}{|c|}{ Men 60-70 $(n=333)^{c}$} & \multicolumn{3}{|c|}{$\operatorname{Men} 71+(n=376)^{c}$} \\
\hline & $\beta$ & SE & $\mathrm{p}$ & $\beta$ & SE & $\mathrm{p}$ & $\beta$ & SE & $\mathrm{p}$ & $\beta$ & SE & $\mathrm{p}$ \\
\hline Percent lean mass $(\%)^{b}$ & -0.070 & $\mathbf{0 . 0 3 0}$ & 0.035 & -0.060 & 0.036 & 0.121 & -0.107 & $\mathbf{0 . 0 2 3}$ & 0.001 & -0.029 & 0.035 & 0.423 \\
\hline \multicolumn{13}{|l|}{ BMI Group $\left(\mathrm{kg} / \mathrm{m}^{2}\right)$} \\
\hline Underweight $<22.0$ & 0.723 & 0.313 & 0.037 & 0.073 & 0.382 & 0.851 & 0.524 & 0.340 & 0.147 & 0.368 & 0.610 & 0.557 \\
\hline $\begin{array}{l}\text { Healthy Weight 22.0-27.0 } \\
\text { (referent) }\end{array}$ & 0.000 & 0.000 & & 0.000 & 0.000 & & 0.000 & 0.000 & & 0.000 & 0.000 & \\
\hline Overweight $>27.0$ & 0.218 & 0.283 & 0.454 & 0.897 & 0.380 & 0.035 & -0.278 & 0.236 & 0.260 & 0.571 & 0.266 & 0.051 \\
\hline $\operatorname{Age}(y)^{\mathrm{d}}$ & -0.012 & 0.026 & 0.642 & 0.105 & 0.024 & 0.001 & -0.032 & 0.033 & 0.340 & 0.128 & 0.042 & 0.009 \\
\hline \multicolumn{13}{|l|}{ Race/Ethnicity } \\
\hline Non-Hispanic White (referent) & 0.000 & 0.000 & & 0.000 & 0.000 & & 0.000 & 0.000 & & 0.000 & 0.000 & \\
\hline Mexican American & -0.274 & 0.453 & 0.555 & -0.948 & 0.500 & 0.080 & -0.345 & 0.389 & 0.391 & 0.269 & 0.448 & 0.559 \\
\hline Other Hispanic & -0.507 & 0.645 & 0.446 & -0.480 & 1.691 & 0.781 & -0.338 & 0.501 & 0.511 & 1.016 & 1.000 & 0.328 \\
\hline Non-Hispanic Black & 0.599 & 0.450 & 0.206 & 0.007 & 0.621 & 0.991 & 0.190 & 0.396 & 0.640 & -0.057 & 0.535 & 0.917 \\
\hline Other (Includes Multi-racial) & -0.001 & 0.483 & 1.000 & 0.192 & 0.536 & 0.726 & 0.048 & 0.887 & 0.958 & 0.651 & 0.861 & 0.463 \\
\hline \multicolumn{13}{|l|}{ Education } \\
\hline Less than 9th grade (referent) & 0.000 & 0.000 & & 0.000 & 0.000 & & 0.000 & 0.000 & & 0.000 & 0.000 & \\
\hline 9th-11th grade & 0.849 & 0.507 & 0.117 & -0.466 & 0.647 & 0.484 & -0.372 & 0.495 & 0.466 & 0.134 & 0.583 & 0.822 \\
\hline High School Grad & 0.010 & 0.287 & 0.972 & -1.150 & 0.539 & 0.052 & -0.326 & 0.511 & 0.534 & 0.312 & 0.477 & 0.525 \\
\hline Some College/AA & 0.146 & 0.279 & 0.609 & -0.906 & 0.592 & 0.149 & -0.297 & 0.354 & 0.416 & 0.172 & 0.415 & 0.685 \\
\hline College Grad or Above & -0.398 & 0.272 & 0.166 & -1.512 & 0.580 & 0.021 & -0.483 & 0.379 & 0.224 & 0.191 & 0.510 & 0.714 \\
\hline \multicolumn{13}{|l|}{ General Health Condition $^{\mathrm{e}}$} \\
\hline Excellent (referent) & 0.000 & 0.000 & & 0.000 & 0.000 & & 0.000 & 0.000 & & 0.000 & 0.000 & \\
\hline Very good & 0.482 & 0.199 & 0.031 & 0.722 & 0.501 & 0.173 & -0.238 & 0.191 & 0.234 & 0.116 & 0.357 & 0.750 \\
\hline Good & 0.735 & 0.345 & 0.052 & 0.892 & 0.395 & 0.041 & 0.747 & 0.341 & 0.047 & 0.215 & 0.428 & 0.624 \\
\hline Fair & 1.813 & 0.418 & 0.001 & 1.836 & 0.426 & 0.001 & 1.220 & 0.567 & 0.050 & 1.578 & 0.608 & 0.022 \\
\hline Poor & 2.436 & 0.596 & 0.001 & 3.416 & 0.654 & 0.000 & 2.786 & 0.801 & 0.004 & 1.942 & 0.796 & 0.029 \\
\hline Physical illness/injury status $(\mathrm{d})^{\mathrm{f}}$ & 0.078 & 0.018 & 0.001 & 0.035 & 0.013 & 0.019 & 0.062 & 0.021 & 0.011 & 0.037 & 0.017 & 0.053 \\
\hline Multiple $\mathrm{R}^{2}$ & \multicolumn{3}{|c|}{0.29} & \multicolumn{3}{|c|}{0.37} & \multicolumn{3}{|c|}{0.22} & \multicolumn{3}{|c|}{0.38} \\
\hline
\end{tabular}


${ }^{\text {a }}$ Physical Limitation Score (PLS) = Index of disability in relation to performing specific functions of everyday living, unassisted: walking 1/4 mile, walking up 10 steps without resting, lifting or carrying $10 \mathrm{lbs}$ and standing from an armless straight chair. For each task a score of "0" was given for "no difficulty," "1" for "some difficulty," and "2" for "much difficulty," "unable to do," "do not do" or "missing." Scores for individual tasks were summed to total the PLS Score: 0-8 (Higher score indicates greater functional limitation)

${ }^{\mathrm{b}}$ Percent lean mass $(\%)$ is an estimate from QDR 4500 fan beam DXA scan lean muscle mass, excluding fat and bone; percent lean body mass $=($ lean muscle mass (g)/total body mass $(\mathrm{g}))^{*} 100$; some DXA values are multiply imputed due to nonrandom missing data

${ }^{\mathrm{c}}$ Analytic $\mathrm{n}$ shown; each person was weighted to account for complex survey design (including oversampling), survey non-response and post-stratification

${ }^{\mathrm{d}}$ Age is reported in years at time of health screening; individuals $>85 \mathrm{y}$ are all topcoded at 85 years of age

e General Health Condition from Current Health Status NHANES Questionnaire (HUQ.010) = "Would you say your general health is..."

${ }^{\mathrm{f}}$ Physical injury/illness status from Current Health Status NHANES Questionnaire (HSQ.470) = "Thinking about your physical health, which includes physical illness and injury, for how many days during the past 30 days was your physical health not good?" (Range $=0-30$ days) 


\section{Discussion, Conclusions and Directions for Future Research}

The main objectives of this research were to examine protein intake and its relationship to lean body mass in a nationally representative sample of adults $19+$ years as well as to investigate the relationship between lean body mass and physical functioning in a nationally representative sample of older adults aged 60+ years. An additional research objective included analyzing the relationship between protein intake and physical functioning in older adults, if the data showed a positive relationship between lean mass and functioning. As demonstrated in Chapter 4, no such relationship was found, thus this final objective was not pursued further.

\subsection{Discussion}

This research was based on data from adults 19 years and older from the NHANES 2003-2004. Descriptive protein intake and body composition data for the participants included in this research revealed a number of interesting trends (Tables 4.1 through 4.4). In general, total grams of protein consumed per day (mean value) decreased with increasing age group in both men and women, with a very large difference of $32.8 \mathrm{~g} / \mathrm{d}$ between men 19-50 (106.15g/d) and men $71+$ years $(75.85 \mathrm{~g} / \mathrm{d})$. While total grams of protein intake per day decreased with increasing age group, the mean protein as a percent of energy and as a percent contributed by animal foods remained relatively consistent between age groups in both men and women. These findings suggest that a decrease in total energy intake, rather than a selective decrease in protein intake in relation to other 
macronutrients, is most responsible for the lower total grams of protein intake seen in older adults.

Ranges between the $5^{\text {th }}$ and $95^{\text {th }}$ percentiles for protein intake variables were relatively wide in both men and women, with a trend observed for a narrower range with increasing age group. This was expected since the youngest age group (19-50 years old) reflected the widest range of chronological years of age (32 years). In all age groups, the range of total grams of protein intake was much wider in men than in women, perhaps partially explained by men at the higher end of intake consuming much greater amounts of protein and total calories when compared with the high end of intake for women. The range between the $5^{\text {th }}$ and $95^{\text {th }}$ percentiles for total grams of protein consumed per day in men and women ages $71+$ years $(34.33 \mathrm{~g}$ to $124.76 \mathrm{~g}$ in men and $30.31 \mathrm{~g}$ to 95.89 grams in women) was more than 20 grams narrower than the range for younger men and women, $19-50$ years $(51.86 \mathrm{~g}$ to $175.09 \mathrm{~g}$ in men and $34.24 \mathrm{~g}$ to $122.07 \mathrm{~g}$ in women). In men, the entire range of protein intake appeared to shift down with age, with a dropping off of both the high and low ends when compared with younger adults. In older women, the high end of intake dropped off dramatically while the low end remained only slightly below the low end for younger adults. Despite these findings, the range of total protein intake for men and women $71+$ years was still large, at 90.43 and 65.58 grams respectively. Thus, the range of protein intake in all age groups of men and women was likely large enough to observe its association, if any, with lean body mass.

Descriptive data for lean body mass measures revealed a substantially lower total and appendicular lean body mass but only a very slightly lower total and appendicular muscle mass index in older age groups of men and women when compared against younger 
groups. This could be explained by the fact that the muscle mass indices accounted for height while the other measures of lean mass did not. Older adults tend to be smaller in stature and hence may have a smaller absolute lean mass. Surprisingly, percent lean body mass was also relatively consistent between age groups in both men and women, with a mere 3-4\% lesser lean mass observed in older adults 71+ years when compared to younger adults 19-50. This finding is unusual, as we would expect a more drastic difference between older and younger adults. These findings may be partially explained by malnourished or underweight older adult participants who had a high percent lean mass due to wasting conditions during which fat is preferentially burned before muscle. For this reason, percent lean body mass does not always correlate positively with health, as an emaciated and a well-muscled person could essentially exhibit identical values.

The range between the $5^{\text {th }}$ and $95^{\text {th }}$ percentiles for all measures of lean body mass tended to narrow with increasing age group in both men and women. As with protein intake, this may be due to the fact that the youngest age group (19-50 years) reflected the widest range of ages and that the physiological differences between men and women in their twenties and those in their forties may be substantial, thus reflecting a wider range of lean mass values. However, we chose our age groups based on standard DRI groupings used to create protein recommendations and were primarily interested in older adults 51-70 and 71+ years. Mean values for all lean mass measures were, as expected, higher in men than in women for all age groups. Ranges for all lean body mass measures $\left(5^{\text {th }}\right.$ to $95^{\text {th }}$ percentile) were again generally wider in men than in women, especially the range for total lean mass in men $71+$ years $(40.52 \mathrm{~kg}$ to $69.40 \mathrm{~kg})$ versus women $71+$ years (30.01kg to $49.22 \mathrm{~kg}$ ). In general, lean body mass ranges in all age groups of men and 
women were likely wide enough to reflect their associations, if any, with protein intake and physical functioning.

The first main question addressed in this research involved the relationship between protein intake and various measures of lean body mass in adults 19+ years, participating in the NHANES 2003-2004. Our hypothesis that a greater protein intake would be significantly related to greater lean mass, after controlling for diet, size and demographic covariables, was correct for some age-gender groups but was seen most often in men (See Table 5.1 below).

Table 5.1 Summary of findings: Statistical significance ${ }^{a}$ of dietary protein (g/day) as a predictor of lean body mass measures in women and men

\begin{tabular}{cccccc}
\hline $\begin{array}{c}\text { Gender } \\
\text { Age Group }\end{array}$ & $\begin{array}{c}\text { Total Lean } \\
\text { Mass }\end{array}$ & $\begin{array}{c}\text { Appendicular } \\
\text { Lean Mass }\end{array}$ & MMI & $\begin{array}{c}\text { Appendicular } \\
\text { MMI }\end{array}$ & $\begin{array}{c}\text { \% Lea } \\
\text { Mass }\end{array}$ \\
Women & & & & & \\
$19-50$ & NS & NS & MS & NS & NS \\
$51-70$ & NS & NS & NS & NS & NS \\
$71+$ & NS & NS & NS & NS & MS
\end{tabular}

\begin{tabular}{llcccc} 
Men & & & & & \\
$19-50$ & MS & MS & S & S & MS \\
$51-70$ & MS & MS & MS & S & NS \\
$71+$ & MS & NS & NS & NS & S \\
\hline
\end{tabular}

${ }^{a} \mathrm{NS}=$ non-significant (p-value $\left.>0.10\right)$

MS = marginally significant $(\mathrm{p}$-value $0.05<\mathrm{p}<0.10)$

$\mathrm{S}=$ significant $(\mathrm{p}$-value $<0.05)$

Protein expressed as total grams of daily intake and as a percent of total daily energy generally showed a significant positive association or at least a positive trend for an association with lean mass in men but not often in women. Protein as a percent contributed by animal foods never exhibited an even marginal association with lean mass in any age-gender group, with the exception of a marginal positive association observed with percent lean mass in men ages $71+$ years ( $p$-value 0.053 ). Protein expressed as total 
grams of daily intake was never significantly related to any of the lean body mass measures in women while protein as a percent of energy, in some age groups of women, was significantly predictive of total and appendicular muscle mass index and percent lean mass. This may suggest that other covariables such as body size or total energy intake may be stronger predictors of lean mass in women when compared against total grams of protein intake.

The lack of solid, consistent data within an age-gender group gives rise to further questions regarding the greatest influencers of lean mass. The size covariables, body mass index and weight, were always strong, significant, positive predictors of lean body mass (p-value $<0.001$ ). Thus, it is possible to infer that lean body mass may be predicted more strongly by size than by specific dietary factors such as protein intake. It is possible that the high predictive value of the size covariable in our analyses overpowered that of our main predictor variable (protein intake), as total body weight and BMI both include lean body mass as part of their values and hence, were always highly significant positive predictors of lean mass. At the same time, size must be controlled for in some way to account for larger persons having greater amounts of lean mass (Gallagher et al. 1997; VanItallie et al. 1990).

In preliminary analyses conducted and presented for the 2010 Experimental Biology Conference, we chose to use height and total fat mass to control for body size, when examining protein and lean mass, in an attempt to avoid using a size covariable that overlapped with our response variable. For example, we were concerned about the overlap of total lean mass and total body mass (weight), as the former is a component of the latter. When height and fat mass controls were used rather than weight or BMI we 
saw similar trends, in that protein appeared to be significantly predictive of lean mass in men but not in women. The differences observed between our preliminary and final analyses were in the strength of associations between protein and lean mass in men. When height and fat mass were used as size controls instead of BMI or weight the associations between protein and lean mass that we did see in men were generally stronger in that they reflected a smaller p-value.

We theorized that either women were not eating enough protein to witness a significant effect on lean mass, the range of protein intake and lean mass within the group of women was not large enough to witness an effect or some other physiological differences in the muscle-building effect of protein existed between men and women (Parker et al. 2010).

The changes seen in the strength of association between protein and lean mass upon changing the size covariables highlights the importance of choosing the most appropriate size control for each model. After much consideration, we chose to use total body mass or BMI as controls for body size in our final analyses. We concluded that, while lean body mass is a component of total body mass, a control for the weight of a person was needed, as larger people naturally have more lean mass. In addition, we decided that it was still possible to witness a range of total lean mass measures at a given body weight and that we would still be able to observe an association between protein intake and lean mass if one existed.

In other published research, body size has been accounted for in different ways. Several studies used height-normalized indices of lean body mass $\left(\mathrm{kg} / \mathrm{m}^{2}\right)$ as response variables, as was done in this research, as a built-in control for stature and weight 
(VanItallie et al. 1990, Lord et al. 2007, Kyle et al. 2003). VanItallie, in research involving the maintenance of lean and fat mass during semi-starvation in a group of men, used fat-free mass index $\left(\mathrm{kg}\right.$ fat-free mass $\left./ \mathrm{m}^{2}\right)$ as a marker of protein energy malnutrition (PEM). He noted that if an index controlling for height were not used, tall subjects with PEM could exhibit values for fat-free mass similar to shorter, well-nourished individuals (VanItallie et al. 1990).

In another published study by Houston and colleagues, height was used to control for size when examining the effect of protein intake on change in total and appendicular lean body mass over a 3-year period in a large group of older adults (Houston et al. 2008). When Gallagher and colleagues used stepwise multiple regression to investigate the effects of individual demographic and physiological variables on appendicular skeletal muscle mass (ALM) in older men and women, they found that stature and weight alone explained a large amount (between 39 and 67\%) of the variance in ALM (Gallagher et al. 1997). As discussed in Chapter 3, height, weight, BMI and height-normalized indices of lean mass were all ways of controlling for body size in our research, with specific size covariables chosen based on their appropriateness to a given model, explained in detail in Chapter 3. While these variables do complicate the interpretation of our data through their potential for overlapping with the lean mass outcome variable and overpowering protein intake as the main predictor variable, they are essential to our models for observing the association between protein and lean body mass at a given size.

While protein was not always a significant predictor of lean mass in our research, it did reflect several positive, non-significant associations $(0.05<$ p-value $<0.10)$, primarily in men. Several other researchers have reported similar findings of positive associations 
between protein intake and lean body mass, although sometimes in both men and women (Houston et al. 2008; Lord et al. 2007; Paddon-Jones et al. 2004b; Symons et al. 2007; Thalacker-Mercer \& Campbell 2008).

As mentioned above and also previously in the literature review, Houston and colleagues studied the association between total protein intake and 3-year changes in total and appendicular lean mass in a large group of older adults. After adjusting for a wide range of relevant covariables, researchers found that participants in the highest quintile of energy-adjusted protein intake (median $18.2 \%$ of energy) lost $40 \%$ less lean mass than those in the lowest quintile (median $11.2 \%$ of energy). In addition to demographic, dietary and physical activity covariables, Houston's study included several covariables that were not included in our research such as height, smoking status, alcohol use, oral steroid use and prevalence of disease. While these may have been interesting covariables to have also considered, we chose only those covariates that were publicly available and most relevant to our investigation to avoid problems with an over-parameterized model, which is a valid precaution to take when analyzing smaller groups of individuals. The observational, longitudinal nature of Houston et al.'s research makes comparison with our study difficult but it does highlight the apparent role of protein in the preservation of lean mass with aging, which is in line with a number of positive associations we found between protein intake and lean mass (Houston et al. 2008).

The second main question addressed in this research involved various measures of lean body mass and their relationship to physical functioning in older adults ages $60+$ years. As mentioned previously, it was decided that since there was no consistently positive relationship between lean mass and functioning, we would not analyze the direct 
relationship between protein and functioning. Our hypothesis that greater lean mass would be significantly, negatively associated with physical limitation score (positively related to functioning) was not only incorrect but generally the exact opposite of what was seen.

With the exception of percent lean mass, all measures of lean body mass were significantly, positively related to physical limitation score in all age-gender groups, thus demonstrating an association between greater lean mass and poorer physical functioning. Percent lean mass was the only lean mass measure reflecting our expectations in that it was negatively related to physical limitation score (positively related to functioning) in men and women 60-70 years, although not at all in those $71+$. Surprisingly, these relationships were observed even after controlling for BMI group which lead us to conclude that, even within a given size range, greater amounts of lean body mass were generally associated with poorer self-reported functioning.

These results are somewhat inconsistent with much other published research that report worse functioning at lower or with decreasing lean body mass (Doherty 2003; Payette et al. 1998; Zoico et al. 2004). On the other hand the research of Janssen and colleagues, who also use NHANES body composition and physical self-report data in their analyses, more closely aligns with ours in that a lower percent lean was generally found to be associated with poorer physical ability (Jannsen et al. 2002).

In his research, Jannsen studied 4500 adults 60 years and older participating in the NHANES III to test the relationship between sarcopenia, or low percent lean mass, and physical disability. Jannsen and colleagues used percent lean body mass from bioelectrical impedance analysis as a main predictor variable. In defining functional 
limitation, Jannsen and researchers used a combination of self-report answers from the NHANES home interview of functioning as well as actual physical performance tests. The self-report items included by researchers to define limitation were very similar to those used in our research and included: Lifting or carrying ten pounds, walking $1 / 4$ mile, walking up ten steps without resting, stooping/crouching/kneeling, standing from an armless straight chair, performing light household chores and preparing meals. Researchers also used self-report questions regarding ability to perform personal care items such as eating, bathing, dressing and shopping and physical performance tests that included ability to walk eight feet, complete five chair stands and balance with the heel of one foot in front of the toes of another. Researchers from this study assigned a score of 1 if no difficulty was reported for a task or if the person was able to complete it and a score of 0 if any difficulty was reported or the person was unable to complete the physical performance task.

In our research, we chose fewer self-report items that we believed to be most important for mobility and independent functioning and assigned scores of 0,1 or 2 to responses rather than dichotomizing them. We were also unable to use actual tests of physical performance, as these were abandoned in more recent cycles of the NHANES. As opposed to bioelectrical impedance analysis, we used the current gold standard for body composition, dual X-ray absorptiometry (DXA), to estimate lean body mass.

Jannsen's study compared the prevalence of functional impairment and disability in those with a normal percent lean mass versus those with class I or class II sarcopenia (within one to two standard deviations and less than two standard deviations of young adult values for percent lean mass, respectively). Similar to our research, Jannsen's study 
also used multiple regression to examine the associations between sarcopenia and measures of functional impairment and disability, as reflected by odds ratios both unadjusted and adjusted for age, race, BMI, comorbidity and health behaviors (alcohol consumption, smoking and physical activity). In general, results showed associations between sarcopenia and increased odds ratios for functional limitations, especially in those with class II sarcopenia when compared against healthy older adults. These results are consistent with our findings in that a lower percent lean mass was generally associated with poorer physical ability.

Payette and colleagues demonstrated findings generally different from ours in a small cross-sectional study of frail elderly women that examined the relationship between lean body mass and strength/functional ability. In this study, researchers tested the predictive ability of estimates of total fat-free mass $(\mathrm{kg})$ from bioelectrical impedance analysis on handgrip strength, isometric knee extensor and elbow flexor strength, the Timed 'Up and Go' walk test and self-reported subscales of health (general, mental, physical, social and emotional health as well as vitality). The study found all direct measures of strength (handgrip and isometric) to be significantly correlated with fat-free mass $(\mathrm{kg})$ but found no such association between fat-free mass and The Timed 'Up and Go,' a measure of gait speed, dynamic balance and flexibility. In addition, researchers found that fat-free mass did not correlate at all with any of the self-reported health subscales. However, selfreported general pain index and vitality were consistently related to strength and functional measures with lesser general pain and better self-reported vitality associated with greater fat-free mass (Payette et al. 1998). 
Like our research, this study was cross-sectional in nature. However, researchers had actual measured strength and functioning data available whereas we had only self-report values to create an index of functioning. The findings from this study differed from ours in that researchers found self-reported health not at all significant in relation to lean mass while we generally observed poorer physical functioning in individuals with greater lean mass. However, this study also took mental, emotional and social health factors into account when creating a health index whereas we chose to focus only on physical outcomes while using general health and physical illness status covariables. As found in Payette's research, physical pain from arthritis or other chronic conditions as well as overall energy and vitality likely play significant roles in predicting self-perceived and actual functioning in older adults, thereby attenuating the relationship between lean mass and functioning (Payette et al. 1998). As mentioned above, we did control for these to the best of our ability through the use of self-reported health covariables, which were almost always highly significant predictors of functioning in our models.

In a larger longitudinal study examining five-year body composition changes and their associations with worsening disability in older adults, Fantin and colleagues found that reduction in appendicular and leg fat-free mass accounted for a nearly two-fold increase in risk for functional decline over time. Thus again opposite from what we found, lean mass appeared to be negatively associated with functional limitation.

Fantin and researchers used DXA scans to obtain estimates of total body and leg fat mass and total, appendicular and leg fat-free mass at baseline, two years and 5 1/2 years. Anthropometric measures and information regarding self-reported disabilities in performing basic and instrumental ADLs, walking $800 \mathrm{~m}$, walking up ten stairs and doing 
heavy housework were also obtained at baseline and at the two follow-up sessions. While longitudinal in nature, this study was similar to ours in that it used DXA measures of lean and fat mass as well as similar self-report measures of functioning (Fantin et al. 2007).

Researchers concluded that, over the $51 / 2$ year span, total, appendicular and leg fatfree mass decreased significantly ( $p$ for trend $<0.001$ ). After adjusting for gender and using logistic regression to examine the effects of age, baseline BMI, number of diseases, baseline walk test, category of weight change, fat-free mass/fat-free mass change and fat mass/fat mass change, researchers found that loss of appendicular and leg fat-free mass were the only significant predictors of worsening disability (Fantin et al. 2007).

Researchers further adjusted for gender and number of diseases in testing the odds of experiencing worsening disability over time and found that the odds of having a worsening in disability score was 2 to $2 \frac{1}{2} 2$ times greater in those having lost leg or appendicular lean mass when compared to those who had not. These results cannot be directly compared with ours, as they measure change over time but they do highlight the seemingly positive role of lean body mass in the preservation of functioning with age and also show that a decline in fat-free mass of the extremities, particularly the legs, is an important risk factor for disability in old age (Fantin et al. 2007). While our research did take into account appendicular lean mass, it may have also been worthwhile to examine the relationship between leg lean mass alone and physical limitation score.

What then, may explain the findings of our research? In our analyses, body mass index (BMI) group, run as a covariable to control for size, was not a significant predictor of physical limitation score in any age-gender group. This was surprising, as we 
expected both underweight and overweight groups to reflect associations with poorer physical functioning. However, it is still possible that individual body mass index, within a BMI group, is partially responsible for physical limitation. In addition, the two selfreported measures of health, which included a rating of current health status and a report of recent days where physical health was not good, were both significantly related to physical limitation score in nearly all age-gender groups. In general, there was a significant relationship seen between greater physical limitation score (lower functioning) and poorer health status. Thus, it is reasonable to infer that body size as well as general health and physical illness/injury status may be stronger predictors of physical limitation in older adults than lean body mass and thus they somewhat attenuated the relationship between lean mass and functioning.

It is possible that in our research and in that of Jannsen et al. a lower percent lean mass was associated with poorer physical functioning in older men and women due to its additional built-in control for size and percent fat. In our research, percent lean was the only measure of lean mass to include total body mass as part of its calculated value (Total lean mass $(\mathrm{kg}) /$ Total body mass $(\mathrm{kg})) * 100$. Thus, this model looked at the amount of lean mass, relative to the amount of body fat as a predictor of functioning. Our other models did not directly take into account body fat. This finding, along with those mentioned previously, led us to also question whether total fat mass and total percent body fat were greater predictors of physical limitation than lean mass. To investigate this question further, additional regression analyses of fat mass and total percent body fat and their relationship to physical limitation score were run, with results included in Appendix D. 
Results from these analyses partially confirmed our additional hypothesis in that total fat mass was significantly, positively related to physical limitation in men ages 60-70 and women 60-70 and 71+ (p-value $<0.05)$ while percent body fat was significantly, positively related to physical limitation in men and women ages 60-70 (p-value $<0.05$ ) when body size, age, race/ethnicity, education, general health and physical illness/injury status were controlled for (see Appendix D). Thus in general it appears that total and percent fat mass may be more important predictors of physical limitation than lean body mass.

While much published research does support our initial hypothesis of lean body mass being positively associated with functioning, other research reports no such association, agreeing more with our additional analyses mentioned above, in that physical functioning may be more closely tied to fat mass and muscle quality (which includes fat infiltration into muscle) rather than lean mass (Sternfeld et al. 2002; Goodpaster et al. 2006; Bouchard et al. 2007).

Sternfeld and colleagues, in a large cross-sectional study of community-dwelling older adults, examined the relationship between lean and fat mass, estimated via bioelectrical impedance, and both physical performance and self-reported limitation. Physical performance measures included tests of grip strength and gait speed while selfreported limitation included questions regarding difficulty in performing ADLs, similar to those used in our research. Researchers classified everyone reporting "a lot of difficulty" or being "unable" to perform one or more of the ADLs as having a limitation and all others as not. Results of this study revealed that higher fat mass was associated with slower walking speed and a greater likelihood of functional limitation while lean mass was not associated with functional or self-report outcomes after adjustment for 
smoking status, physical activity (METS/wk), presence of chronic conditions, age, height, waist circumference and lean or fat mass. A greater lean to fat ratio was also associated with faster walking speed and less likelihood of reported limitation, suggesting that while absolute amount of lean mass may not be significant, it is an important predictor of physical ability, relative to the amount of body fat (Sternfeld et al. 2002). Findings from this study provide a possible explanation for some of the results found in our research, as it may be the case that physical functioning is more dependent upon fat than lean mass.

In another large longitudinal study, again part of the Health, Aging and Body Composition Study, researchers sought to determine the relationship between loss of lean mass and changes in strength over a three-year time period. Total body and leg lean mass as well as total body and percent fat mass were assessed using DXA, while thigh muscle cross-sectional area and muscle attenuation, essentially the density and composition of muscle (including amounts of intramuscular triglycerides), was measured using CT scan. Muscular strength was assessed via isokinetic knee extensor testing while muscle quality was defined as the ratio of strength to lean mass. All measurements were taken at baseline and again at a 3-year follow-up.

After adjusting for smoking status, physical activity, education, income and health status, researchers found that initially healthy older men and women exhibited a threefold greater loss in strength than decline in muscle mass over the course of three years.

Researchers also found that the maintenance or even gain of lean mass over this time did not necessarily prevent losses in strength (Goodpaster et al. 2006). These findings are important to us as they reveal the possibility that muscle quality may be more important 
than muscle quantity in preserving strength into older age and may partially explain why we did not see an association between lean body mass and increased physical functioning ability. As was mentioned in the literature review, aging muscle experiences losses in Type 2 muscle fibers and protective satellite cells as well as decreased nerve innervation and increasing numbers of proinflammatory cytokines, thereby potentially decreasing the functional ability of muscle without reducing its total mass (Goodpaster et al. 2006; Thornell et al. 2003; Verdijk et al. 2009; Dawson et al. 2008).

Bouchard and colleagues further support the theory that fat mass may be more significantly predictive of physical functioning than lean mass in their large-scale observational study of nearly 1800 ambulatory, community-dwelling older adults. Fat and fat-free mass was measured using DXA, from which appendicular fat-free mass and percent body fat were then calculated. Assessments of physical capacity included normal walking speed and timed single-leg balance while other variables used in the models included self-reported physical activity level, age, gender and number of reported diseases (Bouchard et al. 2007).

Researchers in this study individually examined body composition, demographic and lifestyle variables to determine their relatedness to physical capacity (walking speed and balance). They found that in both men and women, age and percent body fat showed the strongest correlation with physical capacity, while fat-free mass was not significantly correlated (Bouchard et al. 2007). As was seen in Goodpaster's work, fat mass or percent body fat may be more predictive of functioning in older adults than lean mass. These studies along with the findings from ours reveal that fat appears to be an important player and needs to be accounted for when examining physical functioning in older adults. 


\subsection{Research Limitations}

There were several limitations to this research that may have affected results. First of all, this research was cross-sectional, which prevented us from investigating direct cause and effect relationships. In addition, while the NHANES examines a nationally representative sample of the U.S. population, it surveys only non-institutionalized persons, meaning that all hospitalized, nursing home and other non-community dwelling individuals were excluded from the survey. This limitation is especially relevant in research examining older adults, as bias exists toward those who are healthier. Similarly, there is always a survival bias present when examining older adults, as those living into old age are among the strongest and healthiest of their generation.

In terms of the NHANES data available for analysis, we were limited by the large amount of missing DXA data that had to be imputed. While the NHANES imputation process was validated, the values are still statistically generated rather than measured, thus creating potential for a discrepancy between imputed measures and actual body composition. The NHANES' replacement of direct measures of muscular strength and endurance with the physical activity monitor (PAM) left us with limited data to use when assessing the relationship between lean body mass and physical strength and functioning. With no direct measure of strength and endurance, we were left to create our own physical limitation outcome measure based on self-report data. While we created the Physical Limitation Score based on methods reported in other published research, we were unable to validate our index. Despite the potential for error with self-report measures, several studies have shown self-report of functioning to be a valid measure of 
actual functional ability in older adults (Alexander et al. 2000; Cress et al. 1995; Hoeymans et al. 1996; Sayers et al. 2004).

In addition, we chose not to control for level of physical activity when assessing the relationship between lean mass and physical functioning as there was no way of separating ability to exercise from choice to exercise. Older adults who chose to exercise more should have exhibited fewer functional limitations but it is possible that a preexisting condition or weakened state rendered them unable to remain active. As with all research involving lean mass and functioning, it is difficult to discern cause and effect.

When examining the relationship between lean body mass and physical functioning in adults ages $60+$ years, there were a relatively small number of subjects in each agegender group. This was due to the fact that we were only investigating adults 60 years and older and were unable to combine two cycles of NHANES data, as the publicly released DXA measures were not consistent between survey cycles.

\subsection{Conclusions and Directions for Future Research}

Despite its limitations, this research did thoroughly investigate the relationship between protein and lean body mass as well as lean body mass and physical functioning. Models were carefully created to account for all reasonable covariables with controls for dietary, body size, demographic and other health measures. While some results were surprising, we did see several positive associations between protein intake and lean body mass in men but not often in women.

In general, we found that protein appeared to be more strongly predictive of lean mass when the lean body mass measure used as the outcome variable included a built-in 
control for size in addition to a size covariable included in the model. Total and appendicular muscle mass indices were height-normalized and percent lean mass included total body mass as part of its value, allowing for the observation of an association between protein and lean mass per unit size. These findings suggest that relative rather than absolute measures of lean mass may be more accurate outcome measures for future research.

We were surprised by our findings of a generally positive relationship between lean body mass and functional limitation. With the exception of percent lean mass, greater lean body mass was significantly associated with poorer functioning. After analyzing our results and considering findings from other published research, we chose to run additional analyses testing the relationship between total fat mass and percent body fat and physical limitation and found that total and percent body fat was significantly predictive of limitation in most age-gender groups. This new finding leads us to believe that fat mass may be just as important, if not more important, in the functional ability of older adults.

Future research is needed that examines the differences between men and women in terms of their anabolic responses to dietary protein. This research found significant positive associations between protein and lean mass in men but not often in women, warranting further investigation into why these gender differences exist.

Research should also continue to investigate protein intake and its relationship to actual tests of functional capacity such as gait speed, balance, and upper and lower body strength tests in the elderly, making sure to account for body size and body fatness. 
Direct measures of strength and muscular endurance are important, in addition to selfreport, for a more thorough assessment of strength and functioning in older adults.

In addition, future researchers may want to consider the use of separate upper and lower extremity indices of function and lean mass estimates to increase the specificity of the relationships between lean mass and functioning. For example, it would be interesting to consider the relationship between leg lean mass and self-reported walking ability or arm and torso lean mass and the ability to lift and carry ten pounds. NHANES DXA files include lean body mass measures by body compartment so it would be possible to examine these associations using the same nationally representative sample of older adults.

Additionally, this research warrants further investigation into the relationship of fat mass to muscular strength, endurance and physical functioning with studies that look at whether an excess of fat or an inadequate amount of lean mass most strongly predicts ability to function independently as an older adult. A ratio of lean to fat mass would be a worthwhile predictor variable to consider when examining the relationship between body composition and functioning. In addition, future researchers should consider not just the quantity but also the quality of muscle, with studies that examine the role intramuscular fat plays in the functional ability of the whole muscle.

It is also important for researchers to continue their quest for establishing specific health reference values for older adults in an effort to improve the practical usefulness of health assessment tools in this age group. In addition to looking for an association, more information is also needed regarding healthy ranges for protein intake and lean body mass measures in older adults, although these values will likely be debated for quite some 
time. In addition to cross-sectional studies, more longitudinal research should be done that follows groups of older adults over time to monitor changes in diet, weight, body composition and functioning.

This research reveals the complexity behind the concept of body composition and the functional implications of lean mass. It highlights a generally positive association between protein intake and lean body mass in men but not often in women and opens the door to further research involving diet, body composition and physical functioning. 


\section{References}

Abellan van Kan, G., Rolland, Y., Bergman, H., Morley, J. E., Kritchevsky, S. B., \& Vellas, B. (2008). The I.A.N.A Task Force on frailty assessment of older people in clinical practice. The Journal of Nutrition, Health \& Aging, 12(1), 29-37. Retrieved July 6, 2009, from http://www.ncbi.nlm.nih.gov/pubmed/18165842.

Alexander, N., Guire, K. Thelen, D, et al. (2000). Self-Reported Walking Ability Predicts Functional Mobility Performance in Frail Older Adults. Journal of the American Geriatric Society, 48, 1408-1413.

Bales, C., \& Ritchie, C. (2009). Handbook of clinical nutrition and aging. Humana Press of Springer Science + Business Media. New York, NY.

Bartali, B., Frongillo, E. A., Bandinelli, S., Lauretani, F., Semba, R. D., Fried, L. P., et al. (2006). Low Nutrient Intake Is an Essential Component of Frailty in Older Persons. J Gerontol A Biol Sci Med Sci, 61(6), 589-593. Retrieved July 6, 2009, from http://biomed.gerontologyjournals.org/cgi/content/61/6/589.

Baumgartner, R. N., Koehler, K. M., Gallagher, D., Romero, L., Heymsfield, S. B., Ross, R. R., et al. (1998). Epidemiology of Sarcopenia among the Elderly in New Mexico. Am. J. Epidemiol., 147(8), 755-763. Retrieved June 15, 2009, from http://aje.oxfordjournals.org/cgi/content//147/8/755.

Berner, L., Prakash, P., Becker, G., Doi, J. (2009). Characterization of protein intakes in older adults in the US (Experimental Biology Conference Poster Abstract). California Polytechnic State University, San Luis Obispo.

Bonnefoy, M., Jauffret, M., Kostka, T., \& Jusot, J. F. (2002). Usefulness of calf circumference measurement in assessing the nutritional state of hospitalized elderly people. Gerontology, 48(3), 162-169. Retrieved June 29, 2009, from http://www.ncbi.nlm.nih.gov/pubmed/11961370.

Bopp, M., Houston , D., Lenchik, L., Easter, L., Kritchevsky, S., \& Nicklas, B. (2008). Lean Mass Loss Is Associated with Low Protein Intake during Dietary-Induced Weight Loss in Postmenopausal Women. Journal of the American Dietetic Association, 108(7), 1216-1220.

Børsheim, E., Bui, Q. T., Tissier, S., Kobayashi, H., Ferrando, A. A., \& Wolfe, R. R. (2008). Effect of Amino Acid Supplementation on Muscle Mass, Strength and Physical Function in Elderly. Clinical nutrition (Edinburgh, Scotland), 27(2), 189-195. doi: 10.1016/j.clnu.2008.01.001. 
Bouchard, D., Beliaeff, S., Dionne, I., Brochu, M. (2007). Fat Mass But Not Fat-Free Mass Is Related to Physical Capacity in Well-Functioning Older Individuals: Nutrition as a Determinant of Successful Aging (NuAge)—The Quebec Longitudinal Study. Journal of Gerontology, 62(12), 1382-1388.

Campbell, W., Trappe, T., Jozsi, A., Kruskal, L., Wolfe, R., \& Evans, W. (2002). Dietary protein adequacy and lower body versus whole body resistive training in older humans. Journal of Physiology, 542(2), 631-642.

Campbell, W. W., Johnson, C. A., McCabe, G. P., \& Carnell, N. S. (2008). Dietary protein requirements of younger and older adults. Am J Clin Nutr, 88(5), 13221329. Retrieved June 16, 2009, from http://www.ajcn.org/cgi/content/88/5/1322.

Castaneda, C., Charnley, J., Evans, W., \& Crim, M. (1995). Elderly women accommodate to a low-protein diet with losses of body cell mass, muscle function, and immune response. American Journal of Clinical Nutrition, 62, 3039.

Centers for Disease Control and Prevention (CDC 2003). National Center for Health Statistics (NCHS). National Health and Nutrition Examination Survey Questionnaire- Physical Functioning Questionnaire. Hyattsville, MD: U.S. Department of Health and Human Services, Centers for Disease Control and Prevention. Retrieved from http://www.cdc.gov/nchs/nhanes/nhanes2003-2004/quex03_04.htm

Centers for Disease Control and Prevention (CDC 2005). National Center for Health Statistics (NCHS). National Health and Nutrition Examination Survey Documentation, Codebook and Frequencies MEC Exam Component: Balance Examination Data. Retrieved from http://www.cdc.gov/nchs/nhanes/nhanes20032004/exam03_04.htm.

Centers for Disease Control and Prevention (CDC 2006). National Center for Health Statistics (NCHS). National Health and Nutrition Examination Survey Documentation, Codebook and Frequencies MEC Exam Component: Physical Activity Monitor Examination Data. Retrieved from http://www.cdc.gov/nchs/nhanes/nhanes2003-2004/exam03_04.htm.

Centers for Disease Control and Prevention (CDC 2007a). National Center for Health Statistics (NCHS). National Health and Nutrition Examination Survey, Overview. Hyattsville, MD: U.S. Department of Health and Human Services, Centers for Disease Control and Prevention. Retrieved from http://www.cdc.gov/nchs/nhanes/about_nhanes.htm 
Centers for Disease Control and Prevention (CDC 2007b). National Center for Health Statistics (NCHS). National Health and Nutrition Examination Survey

Documentation, Codebook and Frequencies- Dietary Interview Individual Foods. Retrieved from http://www.cdc.gov/nchs/nhanes/nhanes20032004/exam03_04.htm.

Centers for Disease Control and Prevention (CDC 2008a). National Center for Health Statistics (NCHS). National Health and Nutrition Examination Survey, Documentation, Codebook, and Frequencies Dual-Energy X-ray Absorptiometry. Hyattsville, MD: U.S. Department of Health and Human Services, Centers for Disease Control and Prevention. Retrieved from http://www.cdc.gov/nchs/nhanes/dxx/dxa.htm.

Centers for Disease Control and Prevention (CDC 2008b). National Center for Health Statistics (NCHS). National Health and Nutrition Examination Survey, Technical Documentation for the 1999-2004 Dual Energy X-Ray Absorptiometry (DXA) Multiple Imputation Data files. Hyattsville, MD: U.S. Department of Health and Human Services, Centers for Disease Control and Prevention. Retrieved from http://www.cdc.gov/nchs/nhanes/dxx/dxa.htm.

Centers for Disease Control and Prevention (CDC 2008c). National Center for Health Statistics (NCHS). National Health and Nutrition Examination Survey, Documentation, Codebook, and Frequencies- Physical Functioning Questionnaire. Hyattsville, MD: U.S. Department of Health and Human Services, Centers for Disease Control and Prevention. Retrieved from http://www.cdc.gov/nchs/nhanes/nhanes2003-2004/quex03_04.htm

Centers for Disease Control and Prevention (CDC) (2009). National Center for Health Statistics (NCHS). National Health and Nutrition Examination,. Cross-Year Variable Tables for Continuous NHANES. Hyattsville, MD: U.S. Department of Health and Human Services, Centers for Disease Control and Prevention. Retrieved from http://www.cdc.gov/nchs/nhanes/nhanes.

Chernoff, R. (2004). Protein and Older Adults. J Am Coll Nutr, 23(suppl_6), 627S-630. Retrieved June 15, 2009, from http://www.jacn.org/cgi/content/23/suppl_6/627S.

Chevalier, S., Gougeon, R., Nayar, K., \& Morais, J. (2003). Frailty amplifies the effects of aging on protein metabolism: role of protein intake. American Journal of Clinical Nutrition, 78, 422-429.

Chumlea, W.C., \& Guo, S. (1992). Equations for Predicting Stature in White and Black Elderly Individuals. J Gerontol, 47(6), M197-203. doi: 10.1093/geronj/47.6.M197. 
Chumlea, W. C., Guo, S. S., Wholihan, K., Cockram, D., Kuczmarski, R. J., \& Johnson, C. L. (1998). Stature prediction equations for elderly non-Hispanic white, nonHispanic black, and Mexican-American persons developed from NHANES III data. Journal of the American Dietetic Association, 98(2), 137-142. doi: $10.1016 / \mathrm{S} 0002-8223(98) 00036-4$.

Clasey, J. L., Kanaley, J. A., Wideman, L., Heymsfield, S. B., Teates, C. D., Gutgesell, M. E., et al. (1999). Validity of methods of body composition assessment in young and older men and women. J Appl Physiol, 86(5), 1728-1738. Retrieved June 29, 2009, from http://jap.physiology.org/cgi/content/86/5/1728.

Corrada, M. M., Kawas, C. H., Mozaffar, F., \& Paganini-Hill, A. (2006). Association of Body Mass Index and Weight Change with All-Cause Mortality in the Elderly. Am. J. Epidemiol., 163(10), 938-949. doi: 10.1093/aje/kwj114.

Cress, E., Schechtman, K., Mulrow, C., Fiatarone, M., Gerety, M., Buchner, D. (1995). Relationship Between Physical Performance and Self-Perceived Physical Function. Journal of the American Geriatric Society, 43, 93-101.

Cruz-Jentoft, A., Baeyens J., Bauer, J., Boirie, Y., Cederholm, T., Landi, F., Martin, F., Michel, J. et al. (2010). Sarcopenia: European consensus on definition and diagnosis. Age and Ageing, 1093(10) 1-12.

Curhan, G. C., Willett, W. C., Rimm, E. B., \& Stampfer, M. J. (1993). A Prospective Study of Dietary Calcium and Other Nutrients and the Risk of Symptomatic Kidney Stones. N Engl J Med, 328(12), 833-838. doi: 10.1056/NEJM199303253281203.

Dawson, B., Taylor, J., \& Favaloro, E. J. (2008). Potential benefits of improved protein intake in older people. Nutrition \& Dietetics, 65(2), 151-156. doi: 10.1111/j.17470080.2008.00250.x.

DeVita, P., \& Hortobagyi, T. (2000). Age causes a redistribution of joint torques and powers during gait. J Appl Physiol, 88(5), 1804-1811. Retrieved July 7, 2009, from http://jap.physiology.org/cgi/content/abstract/88/5/1804.

Dey, D., Rothenberg, E., \& Sundh, V. (1999). Height and body weight in the elderly. I. A 25 -year longitudinal study of a population aged 70 to 95 years. European Journal of Clinical Nutrition, 53(12), 905-914.

Doherty, T. J. (2003). Invited Review: Aging and sarcopenia. J Appl Physiol, 95(4), 1717-1727. doi: 10.1152/japplphysiol.00347.2003.

Ervin, B. (2006, August 23). Prevalence of Functional Limitations Among Adults 60 Years of Age and Over: United States, 1999-2002. Centers for Disease Control. Retrieved June 23, 2009. 
Fantin, F., Di Francesco, V., Fontana, G., Zivelonghi, A., Bissoli, L., Zoico, E., Rossi, A., Micciolo, R., Bosello, O., Zamboni, M. (2007). Longitudinal Body Composition Changes in Old Men and Women: Interrelationships with Worsening Disability. Journal of Gerontology, 62A(12), 1375-1381.

Fulgoni, V. L. (2008). Current protein intake in America: analysis of the National Health and Nutrition Examination Survey, 2003-2004. Am J Clin Nutr, 87(5), 1554S1557. Retrieved June 15, 2009, from http://www.ajcn.org/cgi/content/87/5/1554S.

Gaffney-Stomberg, E., Insogna, K., Rodriguez, N., \& Kerstetter, J. (2009). Increasing dietary protein requirements in elderly people for optimal muscle and bone health. Journal of the American Geriatrics Society, 57(6), 1073-1079. Retrieved July 19, 2009.

Gaillard, C., Emmanuel Alix, Yves Boirie, Gilles Berrut, \& Patrick Ritz. (2008). Are Elderly Hospitalized Patients Getting Enough Protein? Journal of the American Geriatrics Society, 56(6), 1045-1049. doi: 10.1111/j.1532-5415.2008.01721.x.

Gallagher, D., Visser, M., De Meersman, R. E., Sepulveda, D., Baumgartner, R. N., Pierson, R. N., et al. (1997). Appendicular skeletal muscle mass: effects of age, gender, and ethnicity. J Appl Physiol, 83(1), 229-239. Retrieved July 19, 2009, from http://jap.physiology.org/cgi/content/83/1/229.

Gause-Nilsson, I., \& Dey, D. K. (2005). Percent body fat estimation from skin fold thickness in the elderly. Development of a population-based prediction equation and comparison with published equations in 75-year-olds. The Journal of Nutrition, Health \& Aging, 9(1), 19-24. Retrieved June 29, 2009, from http://www.ncbi.nlm.nih.gov/pubmed/15750661.

Gibson, R. (2005). Principles of Nutritional Assessment (2nd ed., pp. 197-220). Oxford University Press.

Goodpaster, B. H., Carlson, C. L., Visser, M., Kelley, D. E., Scherzinger, A., Harris, T. B., et al. (2001). Attenuation of skeletal muscle and strength in the elderly: The Health ABC Study. J Appl Physiol, 90(6), 2157-2165. Retrieved July 8, 2009, from http://jap.physiology.org/cgi/content/90/6/2157.

Goodpaster, B. H., Won Park, S., Harris, T., Kritchevsky, S., Nevitt, M., Schwartz, A., Simonsick, E., Tylavsky, F., Visser, M., Newman, A. (2006) The Loss of Skeletal Muscle Strength, Mass, and Quality in Older Adults: The Health, Aging and Body Composition Study. Journal of Gerontology, 61A(10), 1059-1064. 
Goya Wannamethee, S., Gerald Shaper, A., Whincup, P. H., \& Walker, M. (2004). Overweight and obesity and the burden of disease and disability in elderly men. Int J Obes Relat Metab Disord, 28(11), 1374-1382. Retrieved June 24, 2009, from http://dx.doi.org/10.1038/sj.ijo.0802775.

Guralnik, J., Ferrucci, L., Pieper, C., Leveille, S., Markides, K., Ostir, G., Studenski, S., Berkman, L., Wallace, R. (2000). Lower Extremity Function and Subsequent Disability: Consistency Across Studies, Predictive Models, and Value of Gait Speed Alone Compared With the Short Physical Performance Battery. Journal of Gerontology, 55A(4), M221-M231.

Heaney, R. P. (1998). Excess Dietary Protein May Not Adversely Affect Bone. J. Nutr., 128(6), 1054-1057. Retrieved June 15, 2009, from http://jn.nutrition.org/cgi/content/128/6/1054.

Heyward, V. (2006). Advanced Fitness Assessment and Exercise Prescription (5th ed.). Burgess Publishing Company.

Hoeymans, N., Feskens, E., van den Bos, G., Kromhout, D. Measureing Functional Status: Cross-Sectional and Longitudinal Associations between Performance and Self-Report (Zutphen Elderly Study 1990-1993). (1996). Journal of Clinical Epidemiology, 49(10), 1103-1110.

Houston, D. K., Nicklas, B. J., Ding, J., Harris, T. B., Tylavsky, F. A., Newman, A. B., et al. (2008). Dietary protein intake is associated with lean mass change in older, community-dwelling adults: the Health, Aging, and Body Composition (Health ABC) Study. Am J Clin Nutr, 87(1), 150-155.

Hunt, J. R., Johnson, L. K., \& Fariba Roughead, Z. (2009). Dietary protein and calcium interact to influence calcium retention: a controlled feeding study. Am J Clin Nutr, 89(5), 1357-1365. doi: 10.3945/ajcn.2008.27238.

IOM. (2002). Dietary Reference Intake. Institute of Medicine of the National Academies.

Janssen, I. (2007). Morbidity and Mortality Risk Associated With an Overweight BMI in Older Men and Women[ast]. Obesity, 15(7), 1827-1840. Retrieved June 24, 2009, from http://dx.doi.org/10.1038/oby.2007.217.

Janssen, I., Heymsfield, S., Ross, R. (2002). Low Relative Skeletal Muscle Mass (Sarcopenia) in Older Persons Is Associated with Functional Impairment and Physical Disability. Journal of the American Geriatric Society, 50, 887-896.

Janssen, I., Shepard, D. S., Katzmarzyk, P. T., \& Roubenoff, R. (2004). The healthcare costs of sarcopenia in the United States. Journal of the American Geriatrics Society, 52(1), 80-85. Retrieved June 15, 2009, from http://www.ncbi.nlm.nih.gov/pubmed/14687319. 
Jones, D., Song, X., \& Rockwood, K. (2004). Operationalizing a Frailty Index from a Standardized Comprehensive Geriatric Assessment. Journal of the American Geriatrics Society, 52(11), 1929-1933.

Krieger, J., Sitren, H., Daniels , M., \& Langkamp-Henken, B. (2006). Effects of variation in protein and carbohydrate intake on body mass and composition during energy restriction: A meta-regression. American Journal of Clinical Nutrition, 83, 260274.

Kuczmarski, M. F., Kuczmarski, R. J., \& Najjar, M. (2001). Effects of age on validity of self-reported height, weight, and body mass index: findings from the Third National Health and Nutrition Examination Survey, 1988-1994. Journal of the American Dietetic Association, 101(1), 28-34; quiz 35-36. doi: 10.1016/S00028223(01)00008-6.

Kyle, U., Schutz, Y., Dupertuis, Y., Pichard, C. (2003). Body Composition Interpretation: Contributions of the Fat-Free Mass Index and the Body Fat Mass Index. Nutrition 19:597-604.

Lanza, I. R., Towse, T. F., Caldwell, G. E., Wigmore, D. M., \& Kent-Braun, J. A. (2003). Effects of age on human muscle torque, velocity, and power in two muscle groups. J Appl Physiol, 95(6), 2361-2369. doi: 10.1152/japplphysiol.00724.2002.

Lawlor, D., Bedford, C., Taylor, M., \& Ebrahim, S. (2002). Agreement between measured and self-reported weight in older women. Results from the British Womens' Heart and Health Study. Age and Ageing, 31, 169-174.

Lindle, R. S., Metter, E. J., Lynch, N. A., Fleg, J. L., Fozard, J. L., Tobin, J., et al. (1997). Age and gender comparisons of muscle strength in 654 women and men aged 2093 yr. J Appl Physiol, 83(5), 1581-1587. Retrieved July 7, 2009, from http://jap.physiology.org/cgi/content/83/5/1581.

Locher, J. L., Roth, D. L., Ritchie, C. S., Cox, K., Sawyer, P., Bodner, E. V., et al. (2007). Body Mass Index, Weight Loss, and Mortality in Community-Dwelling Older Adults. J Gerontol A Biol Sci Med Sci, 62(12), 1389-1392. Retrieved July 19, 2009, from http://biomed.gerontologyjournals.org/cgi/content/62/12/1389.

Lord, C., Chaput, J. P., Aubertin-Leheudre, M., Labonté, M., \& Dionne, I. J. (2007). Dietary animal protein intake: association with muscle mass index in older women. The Journal of Nutrition, Health \& Aging, 11(5), 383-387. Retrieved June 15, 2009, from http://www.ncbi.nlm.nih.gov/pubmed/17657359. 
Medline. (2009, July 3). Muscle Biopsy. Retrieved July 3, 2009, from www.nlm.nih.gov/medlineplus/ency/article/003924.htm.

Millward, D. J., \& Roberts, S. B. (1996). Protein Requirements of Older Individuals. Nutrition Research Reviews, 9(01), 67-87. doi: 10.1079/NRR19960006.

Morse, M. H., Haub, M. D., Evans, W. J., \& Campbell, W. W. (2001). Protein Requirement of Elderly Women: Nitrogen Balance Responses to Three Levels of Protein Intake. J Gerontol A Biol Sci Med Sci, 56(11), M724-730. Retrieved July 19, 2009, from http://biomed.gerontologyjournals.org/cgi/content/56/11/M724.

NIH (2009). Aging changes in body shape. In Medline Plus. U.S. National Library of Medicine and the National Institutes of Health.

Otten, J., Pitzi, H., \& Meyers (Eds.). (2006). Dietary Reference Intakes: The Essential Guide to Nutrient Requirements (pp. 3-67, 146-158). Washington D.C.: The National Academies Press. Retrieved June 15, 2009, from http://www.nap.edu/catalog.php?record_id=11537.

Paddon-Jones, D., Sheffield-Moore, M., Urban, R. J., et al. (2004a). Essential Amino Acid and Carbohydrate Supplementation Ameliorates Muscle Protein Loss in Humans during 28 Days Bedrest. J Clin Endocrinol Metab, 89(9), 4351-4358. doi: 10.1210/jc.2003-032159.

Paddon-Jones, D., Sheffield-Moore, M., Zhang, X., et al. (2004b). Amino acid ingestion improves muscle protein synthesis in the young and elderly. Am J Physiol Endocrinol Metab, 286(3), E321-328. doi: 10.1152/ajpendo.00368.2003.

Paddon-Jones, D., Short, K. R., Campbell, W. W., Volpi, E., \& Wolfe, R. R. (2008). Role of dietary protein in the sarcopenia of aging. Am J Clin Nutr, 87(5), 1562S-1566. Retrieved June 15, 2009, from http://www.ajcn.org/cgi/content/87/5/1562S.

Parker, K., Berner, L., Doi, J. (2010). Dietary protein intake among adults in the United States and its relationship to select body composition measures (Experimental Biology Conference Poster Abstract). California Polytechnic State University, San Luis Obispo.

Payette, H., Hanusaik, N., Boutier, V., \& Gray-Donald, K. (1998). Muscle strength and functional mobility in relation to lean body mass in free-living frail elderly women . Eurpean Journal of Clinical Nutrition, 52, 45-53.

Proctor, D. N., O'Brien, P. C., Atkinson, E. J., \& Nair, K. S. (1999). Comparison of techniques to estimate total body skeletal muscle mass in people of different age groups. Am J Physiol Endocrinol Metab, 277(3), E489-495. Retrieved August 4, 2009, from http://ajpendo.physiology.org/cgi/content/277/3/E489. 
Prod'homme, M., Balage, M., Debras, E., Farges, M., Kimball, S., Jefferson, L., et al. (2005, February 15). Differential effects of insulin and dietary amino acids on muscle protein synthesis in adult and old rats. Retrieved June 25, 2009, from http://jp.physoc.org/content/563/1/235.full.

Ramsay, S. E., Whincup, P. H., Shaper, A. G., \& Wannamethee, S. G. (2006a). The Relations of Body Composition and Adiposity Measures to Ill Health and Physical Disability in Elderly Men. Am. J. Epidemiol., 164(5), 459-469. doi: 10.1093/aje/kwj217.

Ramsay, S. E., Whincup, P. H., Shaper, A. G., \& Wannamethee, S. G. (2006b). The Relations of Body Composition and Adiposity Measures to Ill Health and Physical Disability in Elderly Men. Am. J. Epidemiol., 164(5), 459-469. doi: 10.1093/aje/kwj217.

Rand, W. M., Pellett, P. L., \& Young, V. R. (2003). Meta-analysis of nitrogen balance studies for estimating protein requirements in healthy adults. Am J Clin Nutr, 77(1), 109-127. Retrieved June 15, 2009, from http://www.ajcn.org/cgi/content/77/1/109.

Rantanen, T., Guralnik, J. M., Foley, D., Masaki, K., Leveille, S., Curb, J. D., et al. (1999). Midlife Hand Grip Strength as a Predictor of Old Age Disability. JAMA, 281(6), 558-560. doi: 10.1001/jama.281.6.558.

Roberts, S. B., Hajduk, C. L., Howarth, N. C., Russell, R., \& McCrory, M. A. (2005). Dietary Variety Predicts Low Body Mass Indexand Inadequate Macronutrient and MicronutrientIntakes in Community-Dwelling Older Adults. J Gerontol A Biol Sci Med Sci, 60(5), 613-621. Retrieved June 15, 2009, from http://biomed.gerontologyjournals.org/cgi/content/60/5/613.

Roubenoff, R., \& Hughes, V. A. (2000). Sarcopenia: current concepts. The Journals of Gerontology. Series A, Biological Sciences and Medical Sciences, 55(12), M716724. Retrieved June 15, 2009, from http://www.ncbi.nlm.nih.gov/pubmed/11129393.

Rubin, D.(1987). Multiple imputation for nonresponse in surveys. New York: John Wiley and Sons Inc.

Rutledge, B. (2007). DXA Images Can Motivate Weight-Loss Patients. Internal Medicine News, 40(99), 12.

Saladin, K. (2004). Anatomy and Physiology: The Unity of Form and Function (Third., pp. 879-914). McGraw Hill. 
Salamone, L. M., Fuerst, T., Visser, M., Kern, M., Lang, T., Dockrell, M., et al. (2000). Measurement of fat mass using DEXA: a validation study in elderly adults. $J$ Appl Physiol, 89(1), 345-352.

Sayers, S., Brach, J., Newman, A., Heeren, T., Guralnki, J., Fielding, R. (2004). Use of Self-Report to Predict Ability to Walk 400 Meters in Mobility-Limited Older Adults. Journal of the American Geriatric Society, 52, 2099-2103.

Schroeder, E. T., Wang, Y., Castaneda-Sceppa, C., Cloutier, G., Vallejo, A. F., Kawakubo, M., et al. (2007). Reliability of Maximal Voluntary Muscle Strength and Power Testing in Older Men. J Gerontol A Biol Sci Med Sci, 62(5), 543-549.

Scognamiglio, R., Avogaro, A., Negut, C., Piccolotto, R., de Kreutzenberg, S. V., \& Tiengo, A. (2004). The effects of oral amino acid intake on ambulatory capacity in elderly subjects. Aging Clinical and Experimental Research, 16(6), 443-447. Retrieved July 12, 2009, from http://www.ncbi.nlm.nih.gov/pubmed/15739594.

Seidell, J., \& Visscher, T. (2000). Body weight and weight change and their health implications for the elderly. European Journal of Clinical Nutrition, 54(3), S33S39.

Starling, R., Ades, P., \& Poehlman, E. (1999). Physical activity, protein intake, and appendicular skeletal muscle mass in older men. American Journal of Clinical Nutrition, 70, 91-96.

Sternfeld, B., Ngo, L., Satariano, W.A., Tager, I.B. (2002). Associations of body composition with physical performance and self-reported functional limitation in elderly men and women. American Journal of Epidemiology, 156(2), 122-124.

Stookey, J., Adair, L., \& Popkin, B. (2005). Do Protein and Energy Intakes Explain Long-term Changes in Body Composition? The Journal of Nutrition, Health and Aging, 9 (1), 5-17.

Svendsen, O., Haarbo, J., Heitmann, B., Gotfredsen, A., \& Christiansen, C. (1991). Measurement of body fat in elderly subjects by dual-energy x-ray absorptiometry, bioelectrical impedance, and anthropometry. Am J Clin Nutr, 53(5), 1117-1123. Retrieved June 29, 2009, from http://www.ajcn.org/cgi/content/53/5/1117.

Symons, T. B., Schutzler, S. E., Cocke, T. L., Chinkes, D. L., Wolfe, R. R., \& PaddonJones, D. (2007). Aging does not impair the anabolic response to a protein-rich meal. Am J Clin Nutr, 86(2), 451-456. Retrieved July 9, 2009, from http://www.ajcn.org/cgi/content/86/2/451. 
Symons, T. B., Vandervoort, A. A., Rice, C. L., Overend, T. J., \& Marsh, G. D. (2005). Reliability of a Single-Session Isokinetic and Isometric Strength Measurement Protocol in Older Men. J Gerontol A Biol Sci Med Sci, 60(1), 114-119. Retrieved July 12, 2009, from http://biomed.gerontologyjournals.org/cgi/content/60/1/114.

Thalacker-Mercer, A., \& Campbell, W. (2008). Dietary protein intake affects albumin fractional synthesis rate in younger and older adults equally. International Life Sciences Institute. Retrieved July 11, 2009.

Thornell, L., Lindström, M., Renault, V., Mouly, V., \& Butler-Browne, G. S. (2003). Satellite cells and training in the elderly. Scandinavian Journal of Medicine \& Science in Sports, 13(1), 48-55. Retrieved June 15, 2009, from http://www.ncbi.nlm.nih.gov/pubmed/12535317.

U.S. Department of Health and Human Services . (2008). Healthy People 2010: Understanding and imrpoving health--overweight and obesity. U.S. Government Printing Office. Retrieved June 28, 2009.

VanItallie, T., Yang, M., Heymsfield, S., Funk, R., Boileau, R. (1990). Heightnormalized indices of the body's fat-free mass and fat mass: potentially useful indicators of nutritional status. American Journal of Clinical Nutrition, 52:953959.

Verdijk, L. B., Jonkers, R. A., Gleeson, B. G., Beelen, M., Meijer, K., Savelberg, H. H., et al. (2009). Protein supplementation before and after exercise does not further augment skeletal muscle hypertrophy after resistance training in elderly men. Am J Clin Nutr, 89(2), 608-616. doi: 10.3945/ajen.2008.26626.

Villareal, D. T., Apovian, C. M., Kushner, R. F., \& Klein, S. (2005). Obesity in Older Adults: Technical Review and Position Statement of the American Society for Nutrition and NAASO, The Obesity Society[ast][ast]. Obesity, 13(11), 18491863. Retrieved June 15, 2009, from http://dx.doi.org/10.1038/oby.2005.228.

Visscher, T., Seidell, J., Molarius, A., van der Kuip, D., Hofman, A., \& Witteman, J. (2001). A comparison of body mass index, waist-hip ratio and waist circumference as predictors of all-cause mortality among the elderly: the Rotterdam study. International Journal of Obesity, 25(11), 1730-1735. Retrieved June 29, 2009, from http://www.nature.com/ijo/journal/v25/n11/full/0801787a.html.

Visser, M., Fuerst, T., Lang, T., Salamone, L., Harris, T. B., Health, F. T., et al. (1999). Validity of fan-beam dual-energy X-ray absorptiometry for measuring fat-free mass and leg muscle mass. J Appl Physiol, 87(4), 1513-1520. Retrieved July 1, 2009, from http://jap.physiology.org/cgi/content/87/4/1513. 
Visser, M., Pahor, M., Tylavsky, F., Kritchevsky, S. B., Cauley, J. A., Newman, A. B., et al. (2003). One- and two-year change in body composition as measured by DXA in a population-based cohort of older men and women. J Appl Physiol, 94(6), 2368-2374. doi: 10.1152/japplphysiol.00124.2002.

Volpi, E., Mittendorfer, B., Rasmussen, B. B., \& Wolfe, R. R. (2000). The Response of Muscle Protein Anabolism to Combined Hyperaminoacidemia and GlucoseInduced Hyperinsulinemia Is Impaired in the Elderly. J Clin Endocrinol Metab, 85(12), 4481-4490. doi: 10.1210/jc.85.12.4481.

Walrand, S., Short, K. R., Bigelow, M. L., Sweatt, A. J., Hutson, S. M., \& Nair, K. S. (2008). Functional impact of high protein intake on healthy elderly people. Am J Physiol Endocrinol Metab, 295(4), E921-928. doi: 10.1152/ajpendo.90536.2008.

Walrand, S., \& Boirie, Y. (2005). Optimizing protein intake in aging. Current Opinion in Clinical Nutrition and Metabolic Care, 8(1), 89-94. Retrieved June 15, 2009, from http://www.ncbi.nlm.nih.gov/pubmed/15586006.

Wannamethee, S. G., Shaper, A. G., Lennon, L., \& Whincup, P. H. (2007). Decreased muscle mass and increased central adiposity are independently related to mortality in older men . Am J Clin Nutr, 86(5), 1339-1346. Retrieved June 15, 2009, from http://www.ajcn.org/cgi/content/86/5/1339.

Wolfe, R. R. (2008). Protein Summit: consensus areas and future research. Am J Clin Nutr, 87(5), 1582S-1583. Retrieved June 15, 2009, from http://www.ajcn.org.

Wolfe, R. R. (2006). The underappreciated role of muscle in health and disease. Am J Clin Nutr, 84(3), 475-482. Retrieved August 3, 2009, from http://www.ajcn.org/cgi/content/84/3/475.

World Health Organization (WHO). (1998). Obesity: Preventing and Managing the Global Epidemic. World Health Organization. Retrieved June 28, 2009, .

Zoico, E., Di Francesco, V., Guralnik, J. M., Mazzali, G., Bortolani, A., Guariento, S., et al. (2004). Physical disability and muscular strength in relation to obesity and different body composition indexes in a sample of healthy elderly women. Int J Obes Relat Metab Disord, 28(2), 234-241. Retrieved June 15, 2009, from http://dx.doi.org/10.1038/sj.ijo.0802552. 


\section{APPENDICES}

Appendix A: DXA Variables Included in the Multiple Imputation Model

\begin{tabular}{|c|c|}
\hline $\begin{array}{c}\text { Name of Original } \\
\text { Variable }\end{array}$ & Description \\
\hline DXDLAA & Left Arm Area $\left(\mathrm{cm}^{\wedge} 2\right)$ \\
\hline DXDLABMC & Left Arm BMC (g) \\
\hline DXDLAFAT & Left Arm Fat (g) \\
\hline DXDLALER & Left Arm Lean excl BMC (g) \\
\hline DXDRAA & Right Arm Area $\left(\mathrm{cm}^{\wedge} 2\right)$ \\
\hline DXDRABMC & Right Arm BMC (g) \\
\hline DXDRAFAT & Right Arm Fat (g) \\
\hline DXDRALER & Right Arm Lean excl BMC (g) \\
\hline DXDTRFAT & Truncal fat (g) \\
\hline XDTRLER & Trunk Lean excl BMC (g) \\
\hline DXDLLA & Left Leg Area $\left(\mathrm{cm}^{\wedge} 2\right)$ \\
\hline DXDLLBMC & Left Leg BMC (g) \\
\hline DXDLLFAT & Left Leg Fat (g) \\
\hline DXDLLLER & Left Leg Lean excl BMC (g) \\
\hline DXDRLA & Right Leg Area $\left(\mathrm{cm}^{\wedge} 2\right)$ \\
\hline DXDRLBMC & Right Leg BMC (g) \\
\hline DXDRLFAT & Right Leg Fat (g) \\
\hline DXDRLLER & Right Leg Lean excl BMC (g) \\
\hline DXDHEA & Head Area $\left(\mathrm{cm}^{\wedge} 2\right)$ \\
\hline DXDHEBMC & Head Bone Mineral Content (g) \\
\hline DXDHEFAT & Head Fat (g) \\
\hline DXDHELER & Head Lean excl BMC (g) \\
\hline DXDLRA & Left Ribs Area $\left(\mathrm{cm}^{\wedge} 2\right)$ \\
\hline DXDLRBMC & Left Ribs BMC (g) \\
\hline DXDRRA & Right Ribs Area ( $\left.\mathrm{cm}^{\wedge} 2\right)$ \\
\hline DXDRRBMC & Right Ribs BMC (g) \\
\hline DXDTSA & Thoracic Spine Area $\left(\mathrm{cm}^{\wedge} 2\right)$ \\
\hline DXDTSBMC & Thoracic Spine BMC (g) \\
\hline DXDLSA & Lumbar Spine Area $\left(\mathrm{cm}^{\wedge} 2\right)$ \\
\hline DXDLSBMC & Lumbar Spine BMC (g) \\
\hline DXDPEA & Pelvis Area $\left(\mathrm{cm}^{\wedge} 2\right)$ \\
\hline DXDPEBMC & Pelvis BMC (g) \\
\hline
\end{tabular}

Taken from CDC 2008b 


\section{Appendix B: Non-DXA Variables Included in the Imputation Model}

\begin{tabular}{|c|c|c|c|}
\hline $\begin{array}{l}\text { Name of Original } \\
\text { Variable }\end{array}$ & $\begin{array}{l}\text { Analytic Variable } \\
\text { Name }\end{array}$ & Description & Age Range \\
\hline RIDRETH1 & NEWRACE & $\begin{array}{l}\text { Race/Ethnicity } \\
1=\text { Mexican American } \\
2=\text { Non-Hispanic White } \\
3=\text { Non-Hispanic Black } \\
4=\text { Other (includes other Hispanic } \\
\text { and multiracial) }\end{array}$ & $8+$ \\
\hline RIAAGEYR & RIAAGEYR & Age at Screening & $\begin{array}{c}8+ \\
\text { (Although age is } \\
\text { top-coded at } \\
85+\text { years in the } \\
\text { release data files, } \\
\text { continuous age } \\
\text { was used for } \\
\text { those } 80+\text { years.) }\end{array}$ \\
\hline INDFMINC & INCOME & $\begin{array}{l}\text { Annual CPS Family Income } \\
1=<\$ 20,000 \\
2=>=\$ 20,000\end{array}$ & $8+$ \\
\hline DMD140, DMDEDUC & DMDEDUC & $\begin{array}{l}\text { Education- Recode } \\
1=<\text { High School } \\
2=\text { High School diploma } \\
\text { (includes GED) } \\
3=>\text { High School }\end{array}$ & $20+$ \\
\hline $\begin{array}{l}\text { SDASTAND (represents } \\
\text { location of mobile } \\
\text { examination center) }\end{array}$ & METRO & $\begin{array}{l}\text { Indicates if sample person lives } \\
\text { in a metropolitan area } \\
1=\text { Metro } \\
2=\text { Non-Metro area }\end{array}$ & $8+$ \\
\hline $\begin{array}{l}\text { SDASTAND (represents } \\
\text { location of mobile } \\
\text { examination center) }\end{array}$ & REGION & $\begin{array}{l}\text { Indicates region of the country } \\
\text { where sample person lives } \\
1=\text { Northeast } \\
2=\text { Midwest } \\
3=\text { South } \\
4=\text { West }\end{array}$ & $8+$ \\
\hline WTMEC4YR & WTMEC4YR & $\begin{array}{l}\text { Full Sample } 4 \text { Year MEC Exam } \\
\text { Weight (includes only those with } \\
\text { MEC EXAM Weight }>0 \text { ) }\end{array}$ & $8+$ \\
\hline SDDSRVYR & SDDSRVYR & $\begin{array}{l}\text { Data Release Number } \\
1=1999-2000 \\
2=2001-2002 \\
3=2003-2004\end{array}$ & $8+$ \\
\hline BPXDAR & BPXDAR & $\begin{array}{l}\text { Average Diastolic Blood } \\
\text { Pressure }\end{array}$ & $8+$ \\
\hline BPXSAR & BPXSAR & Average Systolic Blood Pressure & $8+$ \\
\hline $\begin{array}{l}\text { BPQ050a, BPQ010, } \\
\text { BPQ020, BPQ040a }\end{array}$ & BPMED & $\begin{array}{l}\text { Now taking prescribed medicine } \\
\text { for blood pressure }\end{array}$ & $16+$ \\
\hline
\end{tabular}




\begin{tabular}{|c|c|c|c|}
\hline & & $\begin{array}{l}1=\text { yes } \\
2=\text { no }\end{array}$ & \\
\hline BMXWT & BMXWT & Weight (kg) & $8+$ \\
\hline BMXARMC & BMXARMC & Arm Circumference $(\mathrm{cm})$ & $8+$ \\
\hline BMXSUB & BMXSUB & Subscapular Skinfold (mm) & $8+$ \\
\hline BMXTRI & BMXTRI & Triceps Skinfold (mm) & $8+$ \\
\hline BMXWAIST & BMXWAIST & Waist Circumference $(\mathrm{cm})$ & $8+$ \\
\hline BMXHT & BMXHT & Height $(\mathrm{cm})$ & $8+$ \\
\hline BMXWAIST_BMXHT & $\begin{array}{l}\text { BMXWAIST_BMX } \\
\text { HT }\end{array}$ & $\begin{array}{l}\text { Waist Circumference } \\
(\mathrm{cm}) / \text { Height }(\mathrm{cm})\end{array}$ & $8+$ \\
\hline BMXBMI & BMXBMI & Body Mass Index $\left(\mathrm{kg} / \mathrm{m}^{* * 2}\right)$ & $8+$ \\
\hline BMXBMI & OBESEIND & $\begin{array}{l}3 \text { BMI categories } \\
1=\text { Under or normal weight }<25 \\
2=\text { Overweight } 25-<30 \\
3=\text { Obese }>=30\end{array}$ & $20+$ \\
\hline DIQ010 & DRDIAB $^{\#}$ & $\begin{array}{l}\text { Doctor told you have diabetes } \\
1=\text { yes } \\
2=\text { no (DIQ } 010=2 \text { or } 3 \text { ) }\end{array}$ & $8+$ \\
\hline HUQ010 & HEALTHSTAT & $\begin{array}{l}\text { General Health Condition } \\
1=\text { excellent/very good } \\
2=\text { good } \\
3=\text { fair/poor }\end{array}$ & $8+$ \\
\hline LBXTC & LBXTC & Total Cholesterol (mg/dL) & $8+$ \\
\hline LBDHDL & LBDHDL & HDL Cholesterol (mg/dL) & $8+$ \\
\hline LBXSTR $^{* * *}$ & LBXSTR & $\begin{array}{l}\text { Natural Log Trigylcerides } \\
(\mathrm{mg} / \mathrm{dL}) \text {-nonfasting }\end{array}$ & $12+$ \\
\hline $\begin{array}{l}\text { BPQ100D, BPQ060, } \\
\text { BPQ080, BPQ090d }\end{array}$ & CHOLMED & $\begin{array}{l}\text { Now taking prescribed medicine } \\
\text { for cholesterol } \\
1=\text { yes } \\
2=\text { no }\end{array}$ & $20+$ \\
\hline PAD200, PAD320 & $\begin{array}{l}\text { PHYSICALACTIVI } \\
\text { TY }\end{array}$ & $\begin{array}{l}\text { Moderate or Vigorous Activity } \\
\text { past } 30 \text { days } \\
1=\text { yes } \\
2=\text { no }\end{array}$ & $12+$ \\
\hline $\begin{array}{l}\text { SMQ620, } \\
\text { SMD630, } \\
\text { SMQ640, } \\
\text { SMQ020, SMQ040 }\end{array}$ & SMOKER & $\begin{array}{l}\text { Smoking Status } \\
1=\text { Never } \\
2=\text { Former } \\
3=\text { Current }\end{array}$ & $12+$ \\
\hline $\begin{array}{l}\text { OSQ030aa to } \\
\text { OSQ030cf, OSQ010a, } \\
\text { OSQ010b, OSQ010c }\end{array}$ & FRACTURE & $\begin{array}{l}\text { Experienced a fracture } \\
1=\text { yes } \\
2=\text { no }\end{array}$ & $20+$ \\
\hline $\begin{array}{l}\text { MCQ250e, MCQ260ea, } \\
\text { MCQ260eb }\end{array}$ & FAMOSTEO & $\begin{array}{l}\text { Family History of Osteoporosis } \\
1=\text { yes } \\
2=\text { no }\end{array}$ & $20+$ \\
\hline $\begin{array}{l}\text { RHQ420, RHQ440, } \\
\text { RHQ510, RHQ520 }\end{array}$ & BCEVER & $\begin{array}{l}\text { Ever take birth control } \\
1=\text { yes } \\
2=\text { no }\end{array}$ & Females $12+$ \\
\hline $\begin{array}{l}\text { RHQ030, RHQ040, } \\
\text { RHQ310, RHQ050, } \\
\text { RHQ060, RHQ340, }\end{array}$ & NEWMENO & $\begin{array}{l}\text { Menopause Status } \\
1=\text { Premenopausal } \\
2=\text { Menopause Transition }\end{array}$ & Females $12+$ \\
\hline
\end{tabular}




\begin{tabular}{|c|c|c|c|}
\hline RHQ290 & & $\begin{array}{l}3=\text { Surgical/ Medical Amenorrhea } \\
4=\text { Natural Menopause }\end{array}$ & \\
\hline $\begin{array}{l}\text { RHQ554, RHQ570, } \\
\text { RHQ580, RHQ596, } \\
\text { RHQ540 }\end{array}$ & ANYEST & $\begin{array}{l}\text { Ever take non-birth control } \\
\text { estrogen } \\
1=\text { yes } \\
2=\text { no }\end{array}$ & Females 20+ \\
\hline ALCDRNKYR & DRNKYR_CAT & $\begin{array}{l}\text { Categories for average alcohol } \\
\text { consumption per year } \\
0=\text { none } \\
1=\text { Less than once a week } \\
2=\text { Less than once a day } \\
3=\text { Once a day or more }\end{array}$ & $20+$ \\
\hline $\begin{array}{l}\text { Nhcode in }(01900, \\
51500,50900,09400)\end{array}$ & OSTEORX & $\begin{array}{l}\text { Osteoporosis Treatments: } \\
\text { alendronate, risedronate, } \\
\text { raloxifene, calcitonin } \\
1=\text { yes } \\
0=\text { no }\end{array}$ & $8+$ \\
\hline fdacode $=1374$ & ANTICONV & $\begin{array}{l}\text { Anticonvulsants } \\
1=\text { yes } \\
0=\text { no }\end{array}$ & $8+$ \\
\hline 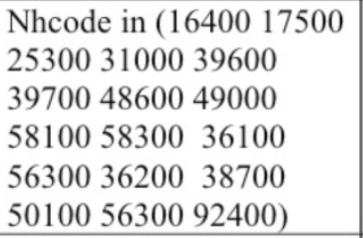 & PRESMEDS & $\begin{array}{l}\text { Prescription Meds: Cortisone, } \\
\text { Thyroid, levothyroxine, liotrix, } \\
\text { methimazole, propylthiouracil } \\
1=\text { yes } \\
0=\text { no }\end{array}$ & $8+$ \\
\hline DSD010, DSDANTA & ANTACID & $\begin{array}{l}\text { Antacids (of those who answered } \\
\text { yes/no to dietary supplement) } \\
1=\text { took antacid past month } \\
2=\text { did not take antacid past } \\
\text { month }\end{array}$ & $8+$ \\
\hline DSD010 & DSD0101 & $\begin{array}{l}\text { Any dietary supplements taken? } \\
1=\text { yes } \\
2=\text { no }\end{array}$ & $8+$ \\
\hline DBD196 & DBD196_NEW & $\begin{array}{l}\text { Past } 30 \text { day milk consumption } \\
\text { recode } \\
1=\text { Never/Rarely less than once a } \\
\text { week } \\
2=\text { Sometimes/Often/Varies }\end{array}$ & $8+$ \\
\hline $\begin{array}{l}\text { DRXTCALC, } \\
\text { DRXTPROT, } \\
\text { DRDTSODI, } \\
\text { DRXTPOTA, } \\
\text { DRXTVC, DRXTZINC, } \\
\text { DRXTMAGN, } \\
\text { DRXTCOPP, } \\
\text { DRXTIRON, } \\
\text { DRXTCAFF }\end{array}$ & $\begin{array}{l}\text { DRXTCALC, } \\
\text { DRXTPROT, } \\
\text { DRDTSODI, } \\
\text { DRXTPOTA, } \\
\text { DRXTVC, } \\
\text { DRXTZINC, } \\
\text { DRXTMAGN, } \\
\text { DRXTCOPP, } \\
\text { DRXTIRON, } \\
\text { DRXTCAFF } \\
\text { DRXTTFAT }\end{array}$ & $\begin{array}{l}\text { Diet: Calcium, protein, sodium, } \\
\text { potassium, vitamin C, zinc, } \\
\text { magnesium, copper, iron, } \\
\text { caffeine, total fat }\end{array}$ & $8+$ \\
\hline
\end{tabular}

Taken from CDC 2008b 
PFQ.030 Is this an impairment or health problem that has lasted, or is expected to last $\mathbf{1 2}$ months or longer?

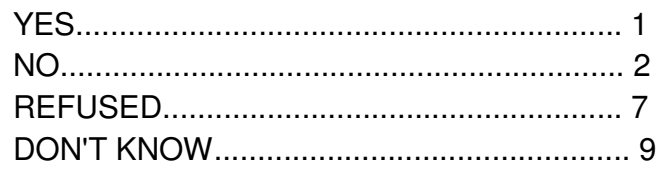

\begin{tabular}{l} 
BOX 1BB \\
CHECK ITEM PFQ.036: \\
IF SP AGE <= 17, CONTINUE. \\
OTHERWISE, GO TO END OF SECTION. \\
\hline
\end{tabular}

PFQ.041 Does $\{$ SP $\}$ receive Special Education or Early Intervention Services?

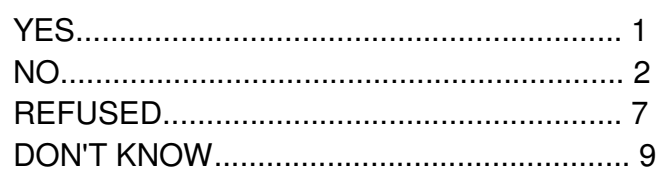

BOX 1C
CHECK ITEM PFQ.045:
GO TO END OF SECTION.

PFQ.049 The next set of questions is about limitations caused by any long-term physical, mental or emotional problem or illness. Please do not include temporary conditions, such as a cold [or pregnancy].

Does a physical, mental or emotional problem now keep \{you/SP\} from working at a job or business?

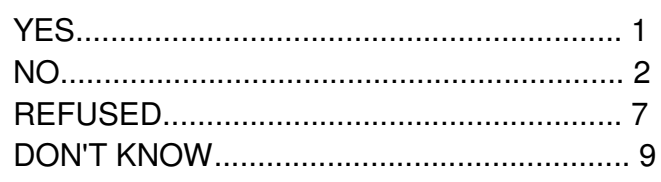

PFQ.051 \{Are you/ls SP\} limited in the kind or amount of work $\{y o u / s / h e\}$ can do because of a physical, mental or emotional problem?

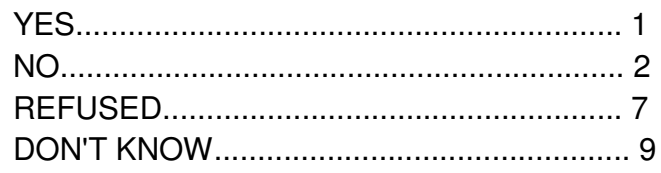


PFQ.054 Because of a health problem, \{do you/does SP\} have difficulty walking without using any special equipment?



PFQ.057 Are you/ls SP\} limited in any way because of difficulty remembering or because $\{$ you/s/he $\}$ experience $\{s\}$ periods of confusion?

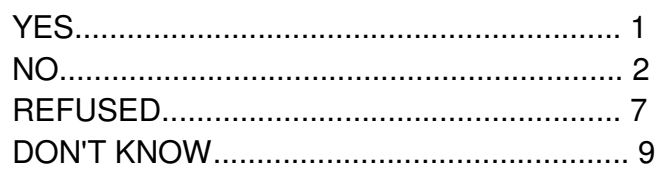

\section{BOX 1D}

CHECK ITEM PFQ.058:

IF 'YES' (CODE 1) IN PFQ.049, PFQ.051, PFQ.054, OR PFQ.057, GO TO PFQ.061.

THERWISE, CONTINUE.

O

PFQ.059 \{Are you/ls SP\} limited in any way in any activity because of a physical, mental or emotional problem?

YES




\section{BOX 1E}

CHECK ITEM PFQ.059A:

IF SP AGE IS <=59 AND 'NO' (CODE 2) ENTERED IN PFQ.049, PFQ.057 AND PFQ.059, GO TO PFQ.090.

OTHERWISE, CONTINUE.

PFQ.061 The next questions ask about difficulties \{you/SP\} may have doing certain activities because of a health problem. By "health problem" we mean any long-term physical, mental or emotional problem or illness \{not including pregnancy\}.

By $\{$ yourself/himself/herself $\}$ and without using any special equipment, how much difficulty $\{$ do you/does SP $\}$ have ...

HAND CARD PFQ1

DO NOT INCLUDE TEMPORARY CONDITIONS LIKE PREGNANCY OR BROKEN LIMBS.

CAPI INSTRUCTION:

IF PFQ.054 = '1' (YES), DO NOT DISPLAY 'B' OR 'C'.

IF SP FEMALE, DISPLAY 'NOT INCLUDING PREGNANCY'.

RESPONSES: NO DIFFICULTY $=1$, SOME DIFFICULTY $=2$, MUCH DIFFICULTY $=3$,

UNABLE TO DO = 4, DO NOT DO THIS ACTIVITY = 5, REFUSED = 7, DON'T KNOW = 9.

a. managing \{your/his/her\} money [such as keeping track of \{your/his/her\} expenses or paying bills]?

b. walking for a quarter of a mile [that is about 2 or 3 blocks]?

c. walking up 10 steps without resting?

d. stooping, crouching, or kneeling?

e. lifting or carrying something as heavy as 10 pounds [like a sack of potatoes or rice]?

f. doing chores around the house [like vacuuming, sweeping, dusting, or straightening up]?

g. preparing \{your/his/her\} own meals?

h. walking from one room to another on the same level?

i. standing up from an armless straight chair?

j. getting in or out of bed?

k. eating, like holding a fork, cutting food or drinking from a glass?

I. dressing \{yourself/himself/herself\}, including tying shoes, working zippers, and doing buttons?

m. standing or being on \{your/his/her\} feet for about 2 hours?

n. sitting for about 2 hours?

o. reaching up over $\{$ your/his/her\} head?

p. using $\{$ your/his/her\} fingers to grasp or handle small objects? 
q. going out to things like shopping, movies, or sporting events?

r. participating in social activities [visiting friends, attending clubs or meetings or going to parties]?

s. doing things to relax at home or for leisure [reading, watching

TV, sewing, listening to music]?

t. push or pull large objects like a living room chair?

\section{BOX 1F}

CHECK ITEM PFQ.066A:

IF 'SOME DIFFICULTY' (CODE 2), 'MUCH DIFFICULTY' (CODE 3), OR 'UNABLE TO DO' (CODE 4) IN PFQ.061 A THROUGH T, CONTINUE.

OTHERWISE, GO TO PFQ.090. 
PFQ.063 What condition or health problem causes $\{y o u / S P\}$ to have difficulty with or need help with $\{$ NAME OF UP TO 3 ACTIVITIES/these activities\}?

HAND CARD PFQ2

ENTER ALL THAT APPLY UP TO 5 BUT DO NOT PROBE.

DO NOT ENTER 'OLD AGE' AS CONDITION -- IF OLD AGE IS REPORTED, PROBE FOR ANY OTHER CONDITION.

CAPI INSTRUCTION:

IF THE TOTAL NUMBER OF ITEMS CODED 'SOME DIFFICULTY' (CODE 2), 'MUCH DIFFICULTY' (CODE 3), OR 'UNABLE TO DO' (CODE 4) IN PFQ.061 A THROUGH T <=3, DISPLAY EACH ITEM NAME IN THE TEXT OF QUESTION. IF MORE THAN 3 ITEMS ARE CODED IN THIS MANNER DISPLAY "THESE ACTIVITIES" IN THE TEXT OF QUESTION.

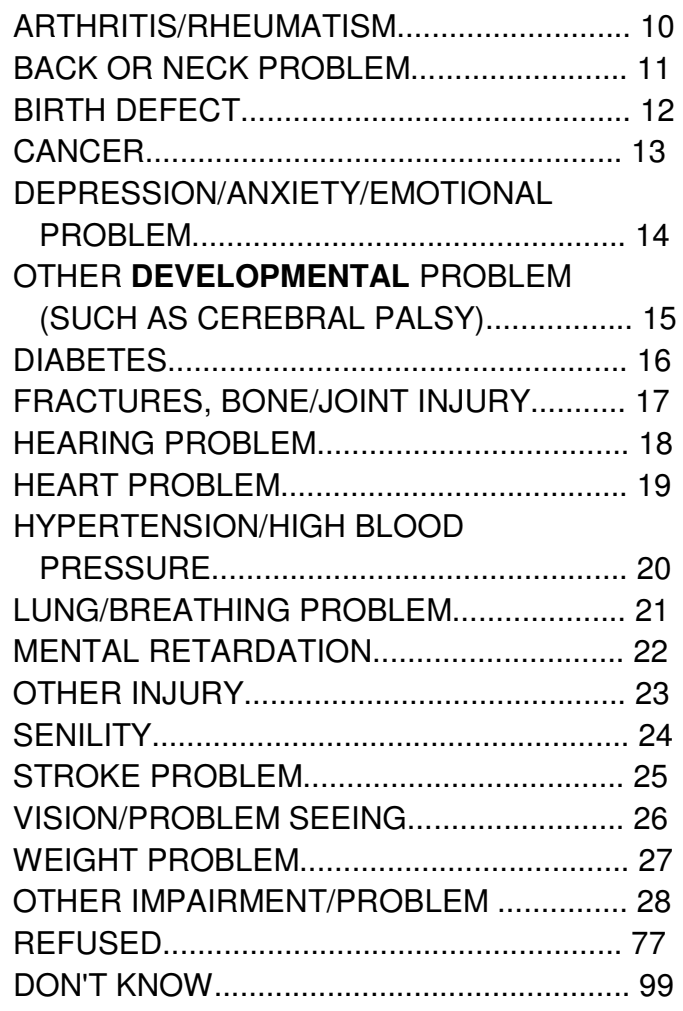




\section{BOX 2}

CHECK ITEM PFQ.068A:

IF CODE 10-11 OR 13-28 IN PFQ.063, CONTINUE WITH LOOP 1.

OTHERWISE, GO TO PFQ.090.

LOOP 1:

ASK QUESTION PFQ.069 FOR EACH CONDITION MENTIONED IN PFQ.063 CONDITION: 10-11 OR 13-28.

PFQ.069 How long have you had \{CONDITION 10-11 or 13-28\}?

CAPI INSTRUCTION:

IF CODE 28 IN PFQ.063, THE FILL SHOULD BE \{THE OTHER CONDITION YOU MENTIONED\}.

ENTER NUMBER (OF DAYS, WEEKS, MONTHS OR YEARS)
SINCE BIRTH
REFUSEDD
DON'T KNOW

\section{BOX 3}

END LOOP 1:

CYCLE ON NEXT CONDITION.

IF NO NEXT CONDITION, GO TO PFQ.090.

PFQ.090 \{Do you/Does SP\} now have any health problem that requires $\{y$ ou/him/her $\}$ to use special equipment, such as a cane, a wheelchair, a special bed, or a special telephone?

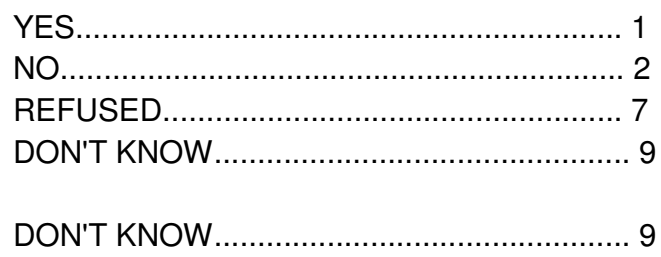

Taken from CDC 2003 


\section{Appendix D: Physical Limitation Score as Predicted by Body Fat Mass}

Physical Limitation Score ${ }^{\mathrm{a}}$ as Predicted by total body fat mass $(\mathrm{kg})^{\mathrm{b}}$

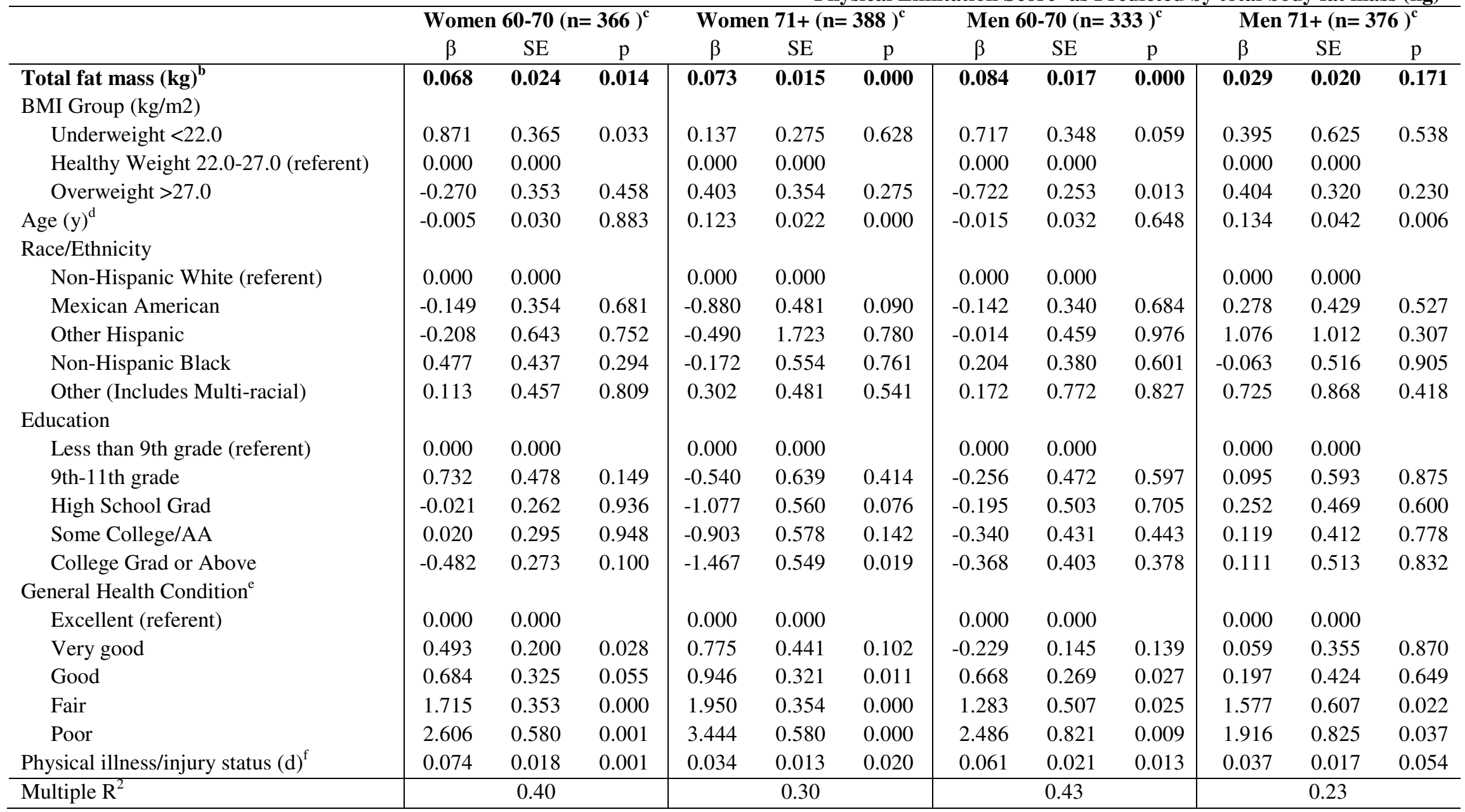


${ }^{\text {a }}$ Physical Limitation Score (PLS) = Index of disability in relation to performing specific functions of everyday living, unassisted: walking 1/4 mile, walking up

10 steps without resting, lifting or carrying $10 \mathrm{lbs}$ and standing from an armless straight chair. For each task a score of "0" was given for "no difficulty," "1" for

"some difficulty," and "2" for "much difficulty," "unable to do," "do not do" or "missing." Scores for individual tasks were summed to total the PLS Score: 0-8 (Higher score indicates greater disability)

${ }^{\mathrm{b}}$ Total body fat mass $(\mathrm{kg})$ is an estimate from QDR 4500 fan beam DXA scan; total body fat mass = all non-lean mass, which excludes muscle and bone; some DXA values are multiply imputed due to nonrandom missing data

${ }^{c}$ Analytic $\mathrm{n}$ shown; each person was weighted to account for complex survey design (including oversampling), survey non-response and post-stratification

${ }^{\mathrm{d}}$ Age is reported in years at time of health screening; individuals $>85 \mathrm{y}$ are all topcoded at 85 years of age

${ }^{\mathrm{e}}$ General Health Condition from Current Health Status NHANES Questionnaire (HUQ.010) = "Would you say your general health is..."

${ }^{\mathrm{f}}$ Physical injury/illness status from Current Health Status NHANES Questionnaire (HSQ.470) = "Thinking about your physical health, which includes physical illness and injury, for how many days during the past 30 days was your physical health not good?" (Range $=0-30$ days) 
Physical Limitation Score $^{\text {a }}$ as Predicted by percent total body fat $(\%)^{\text {b }}$

\begin{tabular}{|c|c|c|c|c|c|c|c|c|c|c|c|c|}
\hline & \multicolumn{3}{|c|}{ Women 60-70 $(n=366)^{c}$} & \multicolumn{3}{|c|}{ Women $71+(n=388)^{c}$} & \multicolumn{3}{|c|}{ Men 60-70 $(n=333)^{c}$} & \multicolumn{3}{|c|}{$\operatorname{Men} 71+(n=376)^{c}$} \\
\hline & $\beta$ & SE & $\mathrm{p}$ & $\beta$ & SE & $\mathrm{p}$ & $\beta$ & $\mathrm{SE}$ & $\mathrm{p}$ & $\beta$ & SE & $\mathrm{p}$ \\
\hline Total percent fat mass $(\%)^{b}$ & 0.074 & 0.030 & 0.026 & 0.058 & 0.033 & 0.108 & 0.112 & 0.024 & 0.000 & 0.019 & 0.035 & 0.590 \\
\hline \multicolumn{13}{|l|}{ BMI Group (kg/m2) } \\
\hline Underweight $<22.0$ & 0.733 & 0.309 & 0.034 & 0.078 & 0.380 & 0.840 & 0.537 & 0.343 & 0.141 & 0.310 & 0.643 & 0.637 \\
\hline Healthy Weight 22.0-27.0 (referent) & 0.000 & 0.000 & & 0.000 & 0.000 & & 0.000 & 0.000 & & 0.000 & 0.000 & \\
\hline Overweight $>27.0$ & 0.185 & 0.279 & 0.519 & 0.894 & 0.370 & 0.031 & -0.349 & 0.244 & 0.177 & 0.607 & 0.284 & 0.053 \\
\hline $\operatorname{Age}(y)^{d}$ & -0.013 & 0.026 & 0.635 & 0.104 & 0.024 & 0.001 & -0.030 & 0.033 & 0.366 & 0.128 & 0.042 & 0.009 \\
\hline \multicolumn{13}{|l|}{ Race/Ethnicity } \\
\hline Non-Hispanic White (referent) & 0.000 & 0.000 & & 0.000 & 0.000 & & 0.000 & 0.000 & & 0.000 & 0.000 & \\
\hline Mexican American & -0.331 & 0.435 & 0.459 & -0.988 & 0.505 & 0.072 & -0.349 & 0.379 & 0.374 & 0.232 & 0.434 & 0.601 \\
\hline Other Hispanic & -0.505 & 0.640 & 0.443 & -0.502 & 1.681 & 0.770 & -0.302 & 0.516 & 0.568 & 1.029 & 1.008 & 0.325 \\
\hline Non-Hispanic Black & 0.617 & 0.447 & 0.190 & 0.051 & 0.598 & 0.934 & 0.220 & 0.389 & 0.581 & -0.072 & 0.539 & 0.896 \\
\hline Other (Includes Multi-racial) & -0.003 & 0.479 & 0.995 & 0.189 & 0.537 & 0.730 & 0.038 & 0.887 & 0.966 & 0.690 & 0.892 & 0.453 \\
\hline \multicolumn{13}{|l|}{ Education } \\
\hline Less than 9th grade (referent) & 0.000 & 0.000 & & 0.000 & 0.000 & & 0.000 & 0.000 & & 0.000 & 0.000 & \\
\hline 9th-11th grade & 0.870 & 0.496 & 0.103 & -0.455 & 0.648 & 0.494 & -0.363 & 0.475 & 0.457 & 0.114 & 0.598 & 0.852 \\
\hline High School Grad & 0.034 & 0.282 & 0.905 & -1.119 & 0.534 & 0.056 & -0.333 & 0.497 & 0.514 & 0.286 & 0.475 & 0.557 \\
\hline Some College/AA & 0.170 & 0.274 & 0.546 & -0.891 & 0.588 & 0.153 & -0.298 & 0.343 & 0.401 & 0.150 & 0.414 & 0.723 \\
\hline College Grad or Above & -0.368 & 0.270 & 0.195 & -1.497 & 0.578 & 0.022 & -0.471 & 0.370 & 0.225 & 0.141 & 0.515 & 0.789 \\
\hline \multicolumn{13}{|l|}{ General Health Condition $^{\mathrm{e}}$} \\
\hline Excellent (referent) & 0.000 & 0.000 & & 0.000 & 0.000 & & 0.000 & 0.000 & & 0.000 & 0.000 & \\
\hline Very good & 0.482 & 0.198 & 0.030 & 0.714 & 0.501 & 0.177 & -0.255 & 0.186 & 0.194 & 0.100 & 0.357 & 0.784 \\
\hline Good & 0.731 & 0.344 & 0.053 & 0.886 & 0.395 & 0.042 & 0.736 & 0.331 & 0.044 & 0.218 & 0.427 & 0.619 \\
\hline Fair & 1.808 & 0.414 & 0.001 & 1.847 & 0.423 & 0.001 & 1.204 & 0.554 & 0.048 & 1.597 & 0.602 & 0.020 \\
\hline Poor & 2.427 & 0.599 & 0.001 & 3.418 & 0.651 & 0.000 & 2.734 & 0.784 & 0.004 & 1.962 & 0.798 & 0.028 \\
\hline Physical illness/injury status $(\mathrm{d})^{\mathrm{f}}$ & 0.078 & 0.018 & 0.001 & 0.034 & 0.013 & 0.020 & 0.062 & 0.021 & 0.012 & 0.036 & 0.017 & 0.053 \\
\hline Multiple $\mathrm{R}^{2}$ & & 0.37 & & & 0.29 & & & 0.38 & & & 0.22 & \\
\hline
\end{tabular}




\footnotetext{
${ }^{a}$ Physical Limitation Score (PLS) = Index of disability in relation to performing specific functions of everyday living, unassisted: walking 1/4 mile, walking up 10 steps without resting, lifting or carrying 10lbs and standing from an armless straight chair. For each task a score of "0" was given for "no difficulty," "1" for "some difficulty," and "2" for "much difficulty," "unable to do," "do not do" or "missing." Scores for individual tasks were summed to total the PLS Score: 0-8 (Higher score indicates greater disability)

${ }^{\mathrm{b}}$ Total percent fat mass (\%) is an estimate from QDR 4500 fan beam DXA scan; total percent fat mass = percent of all non-lean body mass, which excludes muscle and bone; some DXA values are multiply imputed due to nonrandom missing data

${ }^{\mathrm{c}}$ Analytic $\mathrm{n}$ shown; each person was weighted to account for complex survey design (including oversampling), survey non-response and post-stratification

${ }^{\mathrm{d}}$ Age is reported in years at time of health screening; individuals $>85 \mathrm{y}$ are all topcoded at 85 years of age

${ }^{\mathrm{e}}$ General Health Condition from Current Health Status NHANES Questionnaire (HUQ.010) = "Would you say your general health is..."

${ }^{\mathrm{f}}$ Physical injury/illness status from Current Health Status NHANES Questionnaire (HSQ.470) = "Thinking about your physical health, which includes physical illness and injury, for how many days during the past 30 days was your physical health not good?" (Range $=0-30$ )
} 